Supporting Information for

\title{
Coumarin (5,6-Benzo-2-pyrone) Trapping of an HDDA-benzyne
}

\author{
Bhavani Shankar Chinta, Daniel Lee, and Thomas R. Hoye* \\ Department of Chemistry, 207 Pleasant St. SE, University of Minnesota, Minneapolis, MN 55455 \\ email: hoye@umn.edu
}

\section{Table of Contents}

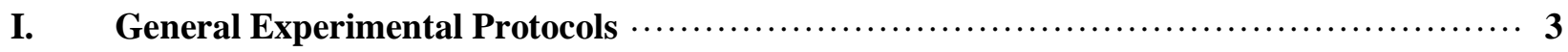

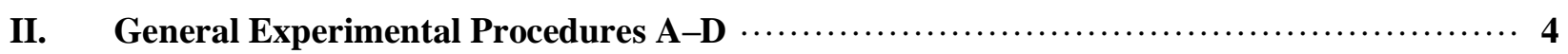

III. Experimental Procedures and Characterization Data for All New Compounds …........ 6

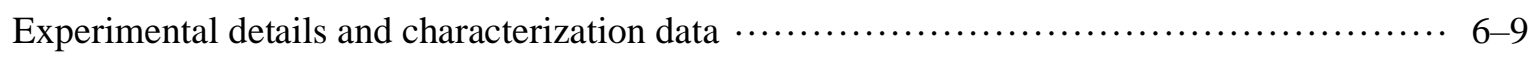

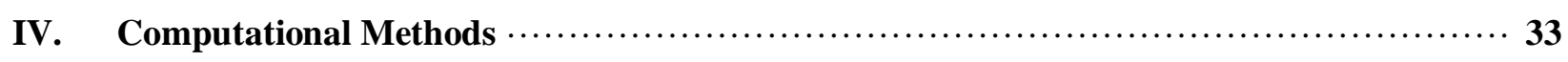

(a) DFT-Calculated energy profiles: reactions of $o$-benzyne with $i$ ) pyrone and ii) coumarin $\cdots 33-43$

(b) DFT-Calculated energy profiles: reactions leading to 16a-anti* and 16a-syn* .......... 44-57

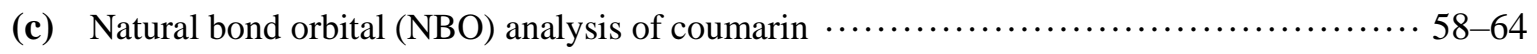

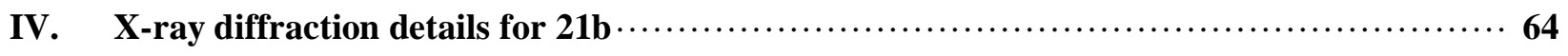

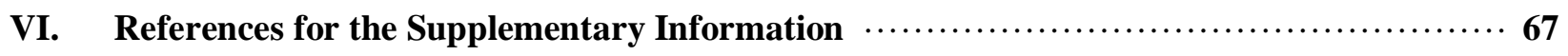

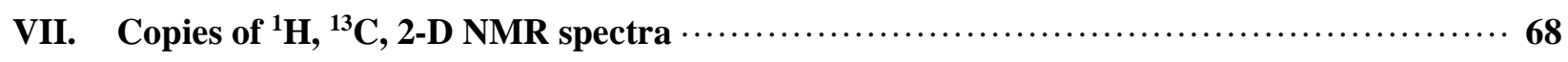

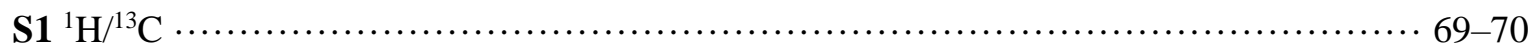

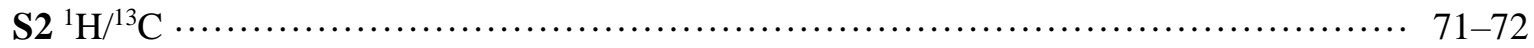

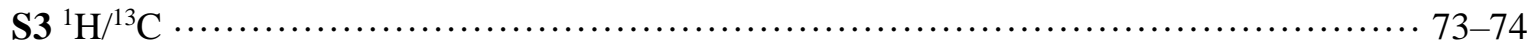

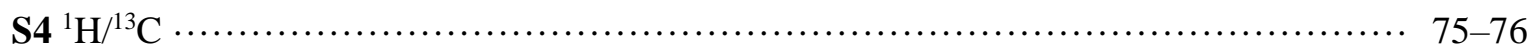

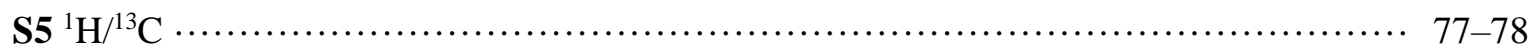

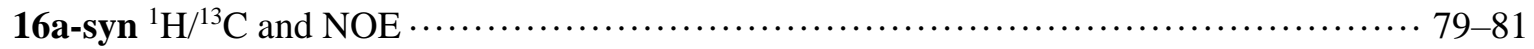

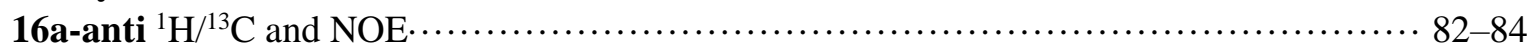

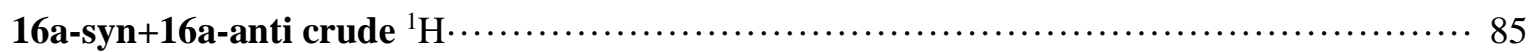

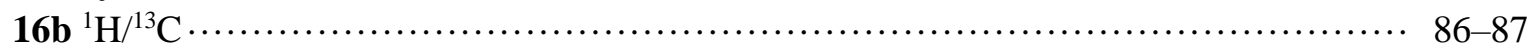

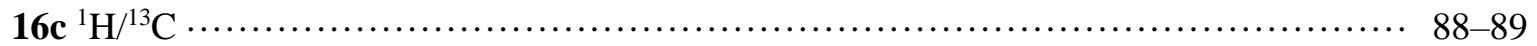

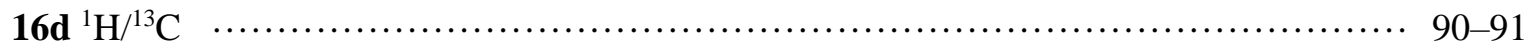

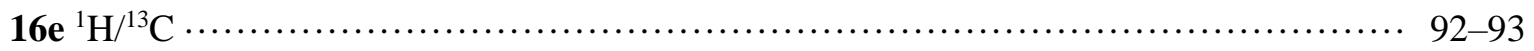

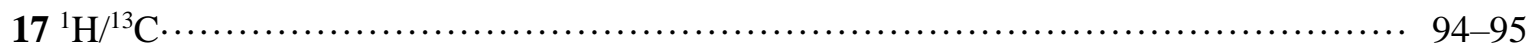

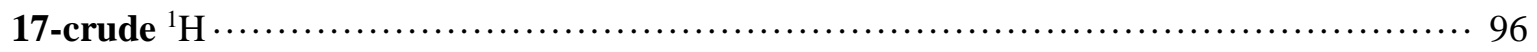




18a ${ }^{1} \mathrm{H} /{ }^{13} \mathrm{C}$
$\mathbf{1 8 b}{ }^{1} \mathrm{H} /{ }^{13} \mathrm{C}$
$\mathbf{1 8 c}$




\section{General Experimental Protocols:}

Nuclear magnetic resonance (NMR) spectra $\left({ }^{1} \mathrm{H}\right.$ and $\left.{ }^{13} \mathrm{C}\right)$ were recorded on a Bruker AX-400 spectrometer. ${ }^{1} \mathrm{H}$ chemical shifts in $\mathrm{CDCl}_{3}$ samples are referenced to TMS $(\delta 0.00)$. Data are reported using the following format: chemical shift (ppm) [multiplicity, coupling constant(s) (in $\mathrm{Hz}$ ), integral (to the nearest whole integer), and assignment of the proton]. Coupling constant values have been deduced using protocols previously described. ${ }^{1,2}$ Non-first order multiplets in a ${ }^{1} \mathrm{H}$ NMR spectrum are designated by 'nfom'. Non-first order doublets in a ${ }^{1} \mathrm{H}$ NMR spectrum (e.g., present in a 1,4-disubstitutedbenzene ring) are designated by 'nfod' and the apparent doublet coupling constant (actually, e.g., $J_{2,3}+J_{2,3}$ ) is indicated as $J_{a p p} .{ }^{13} \mathrm{C}$ NMR chemical shifts are referenced to the carbon atom in $\mathrm{CDCl}_{3}(\delta=77.16)$.

Infrared (IR) spectra were recorded with a Bruker spectrometer (model Alpha II). The samples were deposited as thin films (solids by evaporation of a DCM solution; liquids by direct deposit) on a diamond window using the attenuated total reflectance (ATR) mode. Absorption peak maxima are reported in $\mathrm{cm}^{-1}$.

High-resolution mass spectrometry (HRMS) measurements were determined by ionization in the ESI mode on a Thermo instrument (model Orbitrap Velos having a mass accuracy of $\leq 3$ ). An external calibrant was used (Pierce ${ }^{\mathrm{TM}}$ LTQ). Samples were injected directly into the ion source.

Medium pressure liquid chromatography (MPLC) was used to purify products that eluted close to another by TLC analysis. Silica gel beds (normal-phase, 20-40 $\mu \mathrm{m}, 60 \AA$ pore size, Teledyne RediSep Rf Gold ${ }^{\circledR}$ ) were hand-packed in Michel-Miller ${ }^{\circledR}$ glass columns. The equipment comprised a Waters HPLC pump (model 510), Gilson (111 UV) detector, and Waters (R401) differential refractive index detector. Preparative flash chromatography was done on silica gel columns (Agela, 230-400 mesh). Thin layer chromatography (TLC) was carried out on silica-gel coated, plastic-backed plates (Machery-Nagel) that were visualized by UV light.

Heating of reactions performed above ambient temperature was done in silicone oil baths that had been preequilibrated to the desired temperature prior to immersion of the reaction vessel.

Coumarin derivatives were either commercially available (7a) or prepared from 7hydroxycoumarin (also commercially available) by known methods: $\mathbf{7 b},{ }^{9} \mathbf{7 c},{ }^{10} \mathbf{7 d},{ }^{11}$ and $\mathbf{7 e} .{ }^{12}$ 


\section{General Procedures A-D.}

\section{A. General procedure for the Cadiot-Chodkiewicz cross-coupling reaction}

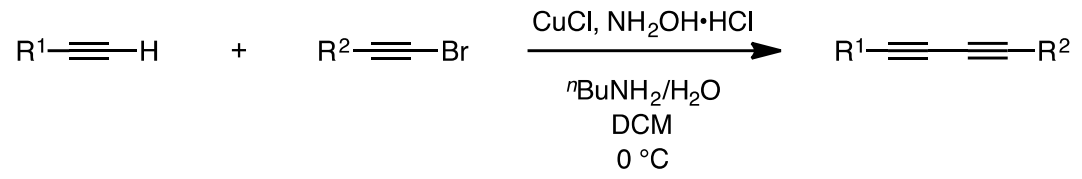

To a solution of $\mathrm{CuCl}(0.050-0.10$ equiv with respect to the terminal alkyne substrate) in $30: 70(\mathrm{v}: \mathrm{v})$ $\mathrm{nBuNH}_{2}: \mathrm{H}_{2} \mathrm{O}(5.0 \mathrm{~mL} / \mathrm{mmol}$ with respect to the terminal alkyne substrate) was added an excess of $\mathrm{NH}_{2} \mathrm{OH} \cdot \mathrm{HCl}$ (typically a few crystals) with stirring. The color of this mixture turned from deep blue to colorless immediately, indicating full conversion of $\mathrm{Cu}$ (II) to $\mathrm{Cu}(\mathrm{I})$. The headspace of the reaction flask was purged with $\mathrm{N}_{2}$. The vessel was sealed with a septum, a nitrogen balloon was attached, and the vessel was cooled in an ice water bath. A solution of the terminal alkyne (1.0 equiv) in $\mathrm{CH}_{2} \mathrm{Cl}_{2}$ (ca. $2.5 \mathrm{~mL} / \mathrm{mmol}$ ) was added into the flask via syringe resulting in a yellow, orange, or red suspension, which is indicative for the formation of an alkynyl copper species. After stirring for ca. 5 min, the 1-bromoalkyne (0.90-1.5 equiv) in $\mathrm{CH}_{2} \mathrm{Cl}_{2}$ (ca. $2.5 \mathrm{~mL} / \mathrm{mmol}$ ) was added dropwise via syringe over ca. 15 min using a syringe pump. The mixture was then kept stirring at the indicated temperature $\left(0{ }^{\circ} \mathrm{C}\right.$ or $\left.\mathrm{rt}\right)$. Typically, the suspension of the alkynyl copper turned clear over the course of 10-100 min, which indicated consumption of the alkynyl copper species. The mixture was quenched by the addition of saturated aqueous $\mathrm{NH}_{4} \mathrm{Cl}$ and extracted with $\mathrm{CH}_{2} \mathrm{Cl}_{2}$. The extracts were dried and concentrated. The crude material was subsequently purified by flash chromatography on silica gel."3

\section{B. General procedure for the oxidation of secondary propargylic alcohols}
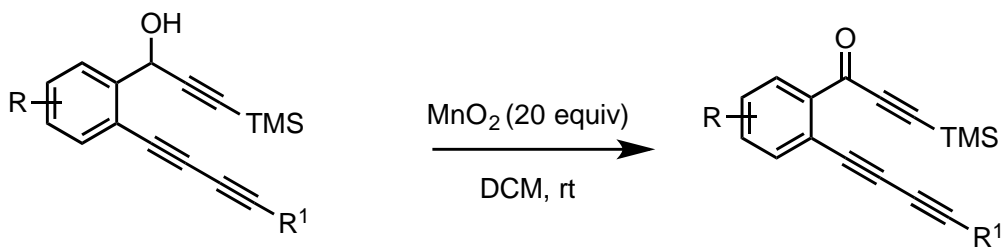

To a stirred solution of the secondary propargylic alcohol (1 equiv) in DCM, activated manganese dioxide (20 equiv) was added. After stirring for 2-4 hours, the reaction mixture was filtered by passing through a Celite ${ }^{\circledR}$ plug and the filtrate was concentrated. Purification of the residue by flash chromatography (hexanes:EtOAc 4:1) provided the corresponding triynone, typically in yields ranging from $80-95 \%$. 


\section{General procedure for the preparation of indenophenanthrenone and indenochrysenone derivatives}
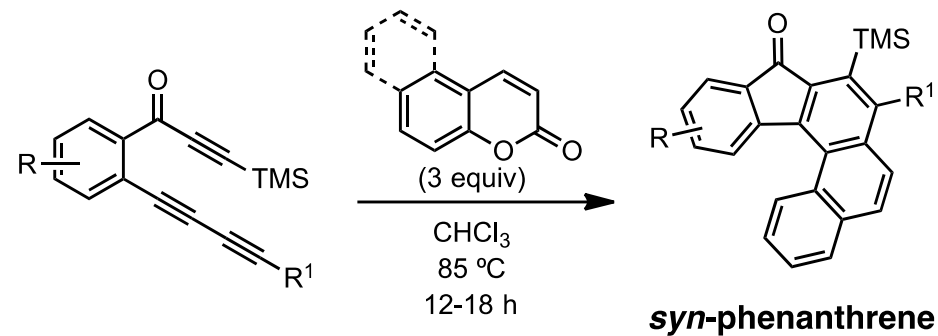

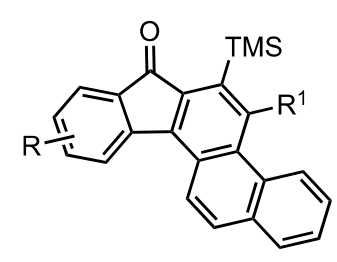

anti-phenanthrene

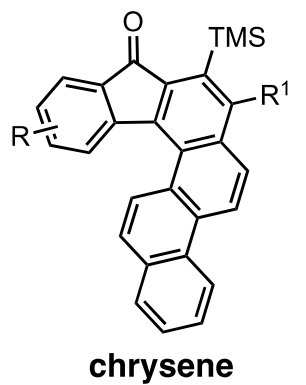

chrysene

A triynone substrate (1.0 equiv) and a coumarin derivative trapping agent (3.0 equiv) were combined in a culture tube, dissolved in. typically, $\mathrm{CHCl}_{3}(3 \mathrm{~mL})$, and sealed with a Teflon-lined cap. When used, the chloroform solvent was stabilized by amylene and not the more commonly used ethanol, because alcohols will competitively react with HDDA benzynes. The solution was heated overnight (12-19 h) in an oil bath at, typically, $85^{\circ} \mathrm{C}$ and then cooled to room temperature. The residue was purified by MPLC (9:1 Hex:EtOAc) to give the corresponding polycylic ketones in, typically, 25-45\% yield.

\section{D. “General procedure for bromination of a terminal- or a TMS-alkyne}

$$
\begin{gathered}
\mathrm{R}=\mathrm{H} \\
\mathrm{or} \\
\mathrm{R}=\mathrm{TMS}
\end{gathered}
$$

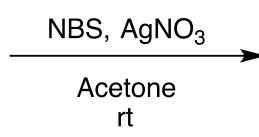<smiles>[R]C#CBr</smiles>

To a stirred solution of terminal alkyne or TMS-protected terminal alkyne (1.0 equiv) and $\mathrm{N}$ bromosuccinimide (NBS, 1.1 equiv) in acetone $(0.10 \mathrm{M})$, powdered $\mathrm{AgNO}_{3}$ (0.10 equiv) was added. After being stirred at room temperature for 1-2 hours (TLC monitoring), an equal volume of hexanes was added to the suspension, and the solid succinimide was removed by filtration through Celite ${ }^{\circledR}$. Following solvent removal from the filtrate, the crude material was purified by flash chromatography."3 


\section{Experimental Procedures and Characterization Data for All New Compounds}

\section{1-(2-((4-Fluorophenyl)buta-1,3-diyn-1-yl)-4,5-dimethoxyphenyl)-3-(trimethylsilyl)prop-2-} yn-1-ol (S1)<smiles>C#Cc1cc(OC)c(OC)cc1C(O)C#C[Na]</smiles><smiles>Fc1ccc(C#CBr)cc1</smiles><smiles>COc1cc(C#CC#Cc2ccc(F)cc2)c(C(O)C#CC#N)cc1OC</smiles>

Following general procedure A: 1-(2-Ethynyl-4,5-dimethoxyphenyl)-3- (trimethylsilyl) (200 mg, $0.69 \mathrm{mmol}$ ) and 1-(bromoethynyl)-4-fluorobenzene ${ }^{4}$ (199 mg, $0.89 \mathrm{mmol}$ ), CuCl (7 mg, 0.069 mmol), $n$-butylamine $/ \mathrm{H}_{2} \mathrm{O}(\mathrm{v}: \mathrm{v}, 30: 70,10 \mathrm{~mL}), \mathrm{NH}_{2} \mathrm{OH} \bullet \mathrm{HCl}(\approx 20 \mathrm{mg})$, and DCM $(10 \mathrm{~mL})$ were used to prepare the triyne S1. Purification of the crude material by flash chromatography (hexanes:EtOAc 4:1) provided triyne $\mathbf{S 1}(200 \mathrm{mg}, 0.492 \mathrm{mmol}, 71 \%)$ as a yellow oil.

${ }^{1} \mathbf{H}$ NMR (400 MHz, $\left.\mathrm{CDCl}_{3}\right): \delta=7.50(\mathrm{app} \mathrm{dd}, J=9.0 \mathrm{HH}, 5.4 \mathrm{HF} \mathrm{Hz}, 2 \mathrm{H}, H 19$ and H23), 7.26 (s, 1H, H6), 7.04 (app dd, $J=8.9 \mathrm{HH}, 8.6 \mathrm{HF} \mathrm{Hz,} \mathrm{2H,H2O} \mathrm{and} \mathrm{H22),} 7.00$ (s, 1H, H3), 5.84 (d, $J=4.9$ $\mathrm{Hz}, 1 \mathrm{H}, \mathrm{ArCHOH}), 3.93$ (s, 3H, CH3 $\mathrm{O}$ ), 3.88 (s, 3H, $\left.\mathrm{CH}_{3} \mathrm{O}^{\prime}\right), 2.38$ (d, J=5.4 Hz, 1H, OH), and $0.21\left(\mathrm{~s}, 9 \mathrm{H}, \mathrm{Si}\left(\mathrm{CH}_{3}\right)_{3}\right)$.

${ }^{13} \mathrm{C}$ NMR (100 MHz, $\left.\mathrm{CDCl}_{3}\right): \delta=163.2\left(\mathrm{~d}, \mathrm{C}-\mathrm{F},{ }^{1} \mathrm{~J}=252 \mathrm{~Hz}\right), 150.6,148.8,137.5,134.6(\mathrm{~d}, \mathrm{C}-$ $\left.\mathrm{F},{ }^{3} J=8.7 \mathrm{~Hz}\right), 118.0\left(\mathrm{~d}, \mathrm{C}-\mathrm{F},{ }^{4} J=3.6 \mathrm{~Hz}\right), 116.1\left(\mathrm{~d}, \mathrm{C}-\mathrm{F},{ }^{2} J=22.1 \mathrm{~Hz}\right), 115.3,112.3,110.1$, $104.5,91.9,81.6,79.0,77.6,73.8,63.2,56.2,56.1$, and -0.1 .

IR (neat): 3483, 3076, 3004, 2958, 2936, 2902, 2856, 2836, 2212, 2172, 2143, 1597, 1570, 1250, $1230,1114,1014$, and $736 \mathrm{~cm}^{-1}$.

HRMS (ESI) m/z: $[\mathrm{M}+\mathrm{H}]^{+}$Calcd for $\mathrm{C}_{24} \mathrm{H}_{24} \mathrm{FO}_{3} \mathrm{Si}$ 407.1459; Found 407.1479. 


\section{4-((2-(1-Hydroxy-3-(trimethylsilyl)prop-2-yn-1-yl)-4,5-dimethoxyphenyl)buta-1,3-diyn-1-} yl)benzonitrile (S2)

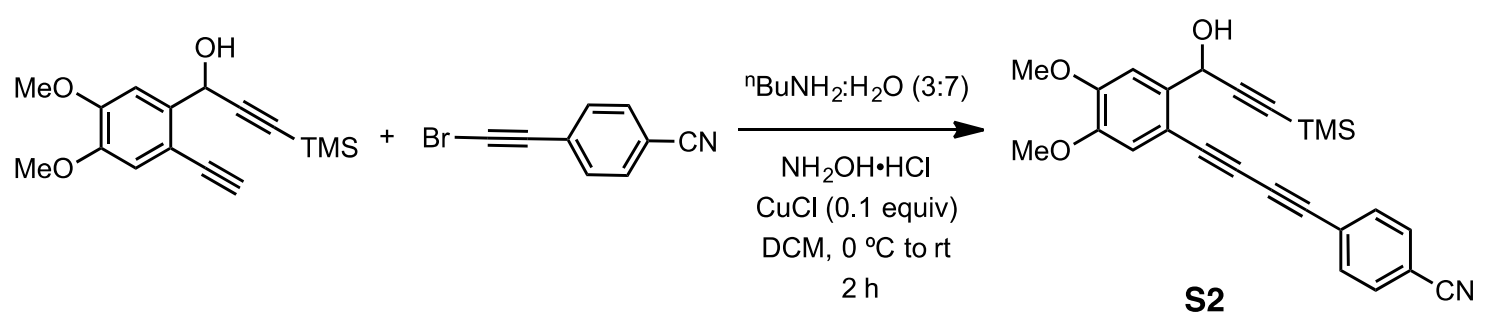

Following general procedure A: 1-(2-Ethynyl-4,5-dimethoxyphenyl)-3-(trimethylsilyl)prop-2yn-1-ol (200 mg, $0.69 \mathrm{mmol})$ and 1-(bromoethynyl)-4-cyanobenzene ${ }^{5}$ (183 mg, $\left.0.89 \mathrm{mmol}\right), \mathrm{CuCl}$ (7 mg, $0.069 \mathrm{mmol}), n$-butylamine/ $\mathrm{H}_{2} \mathrm{O}(\mathrm{v}: \mathrm{v}, 30: 70,10 \mathrm{~mL})$, and DCM (10 mL) were used to prepare triyne S2. Purification of the crude material by flash chromatography (hexanes:EtOAc 4:1) provided the triyne $\mathbf{S 2}$ (230 $\mathrm{mg}, 0.556 \mathrm{mmol}, 81 \%$ ) as a yellow amorphous solid.

${ }^{1} \mathbf{H}$ NMR $\left(400 \mathrm{MHz}, \mathrm{CDCl}_{3}\right.$ ): $\delta=7.63$ (nfod, $J_{a p p}=8.3 \mathrm{~Hz}, 2 \mathrm{H}, \mathrm{ArH19/23}$ or ArH20/22), 7.59 (nfod, $J_{a p p}=8.4 \mathrm{~Hz}, 2 \mathrm{H}, \operatorname{ArH19/23}$ or ArH20/22), 7.27 (s, 1H), 7.00 (s, 1H), 5.82 (s, 1H), 3.95 (s, $\left.3 \mathrm{H}, \mathrm{CH}_{3} \mathrm{O}\right), 3.89$ (s, 3H, $\left.\mathrm{CH}_{3} \mathrm{O}^{\prime}\right)$, and 0.20 (s, 9H, $\left.\mathrm{Si}\left(\mathrm{CH}_{3}\right)_{3}\right)$.

${ }^{13} \mathrm{C}$ NMR (100 MHz, $\left.\mathrm{CDCl}_{3}\right): \delta=151.0,148.9,137.9,133.0,132.3,126.9,118.4,115.4,112.5$, $111.7,110.2,104.4,92.1,81.6,80.5,78.3,77.5,63.2,56.3,56.1$, and -0.1 .

IR (neat): 3482, 3088, 3061, 3005, 2959, 2937, 2904, 2857, 2837, 2227, 2210, 2179, 1600, 1511, 1499, 1405, 1352, and $759 \mathrm{~cm}^{-1}$.

HRMS (ESI) m/z: [M + Na $]^{+}$Calcd for $\mathrm{C}_{25} \mathrm{H}_{23} \mathrm{NO}_{3} \mathrm{NaSi} 436.13$; Found 436.1340. 


\section{1-(4-((2-(1-Hydroxy-3-(trimethylsilyl)prop-2-yn-1-yl)-4,5-dimethoxyphenyl)buta-1,3-diyn-} 1-yl)phenyl)ethanone (S3)

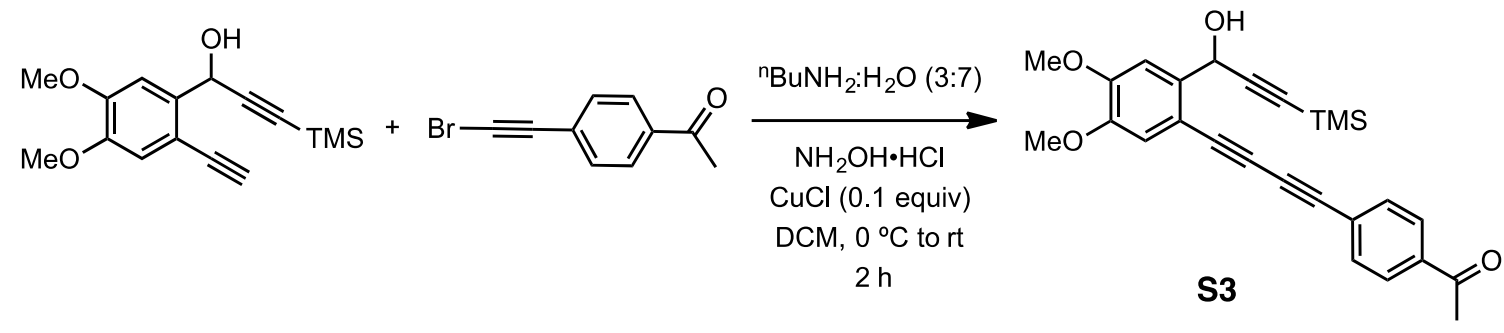

Following general procedure A: 1-(2-Ethynyl-4,5-dimethoxyphenyl)-3-(trimethylsilyl)prop-2yn-1-ol (200 mg, $0.69 \mathrm{mmol})$ and 1-(4-(bromoethynyl)phenyl)ethanone 5 (200 mg, $0.89 \mathrm{mmol}$ ), $\mathrm{CuCl}$ (7 mg, $0.069 \mathrm{mmol})$, n-butylamine/ $\mathrm{H}_{2} \mathrm{O}$ (v:v, 30:70, $\left.10 \mathrm{~mL}\right)$, and DCM (10 mL) were used to prepare triyne S3. Purification of the crude material by flash chromatography (hexanes: EtOAc 4:1) provided the triyne $\mathbf{S 3}$ (140 $\mathrm{mg}, 0.325 \mathrm{mmol}, 47 \%$ ) as a yellow crystalline solid.

${ }^{1} \mathbf{H}$ NMR (400 MHz, $\left.\mathrm{CDCl}_{3}\right): \delta=7.94\left(\right.$ nfod, $J_{a p p}=8.6 \mathrm{~Hz}, 2 \mathrm{H}, \mathrm{ArH1}$ \& ArH23), 7.61 (nfod, $J_{a p p}$ $=8.4 \mathrm{~Hz}, 2 \mathrm{H}, \mathrm{ArH} 20$ \& ArH22), 7.29 (s, 1H, H6), 7.01 (s, 1H, H3), 5.86 (br s, 1H, CHOH), 3.96 (s, 3H, $\mathrm{CH}_{3} \mathrm{O}$ ), 3.90 (s, 3H, $\mathrm{CH}_{3} \mathrm{O}$ '), 2.63 (d, $\left.J=4 \mathrm{~Hz}, 1 \mathrm{H}, \mathrm{OH}\right), 2.62$ (s, 3H, $\mathrm{COCH}_{3}$ ), and 0.22 $\left(\mathrm{s}, 9 \mathrm{H}, \mathrm{Si}\left(\mathrm{CH}_{3}\right)_{3}\right)$.

${ }^{13} \mathrm{C}$ NMR (100 MHz, $\left.\mathrm{CDCl}_{3}\right): \delta=197.3,150.8,148.8,137.8,136.9,132.6,128.4,126.7,115.3$, $111.9,110.1,104.5,91.9,81.6,80.8,77.45,77.43$ 63.2, 56.2, 56.1, 26.8, and -0.1 .

IR (neat): 3488, 3003, 2959, 2937, 2905, 2856, 2836, 2209, 1683, 1355, and $841 \mathrm{~cm}^{-1}$. HRMS (ESI) m/z: [M + H] ${ }^{+}$Calcd for $\mathrm{C}_{26} \mathrm{H}_{27} \mathrm{O}_{4} \mathrm{Si} 431.1679$; Found 431.1670. mp: $142-145^{\circ} \mathrm{C}$. 


\section{1-(4,5-Dimethoxy-2-((4-methoxyphenyl)buta-1,3-diyn-1-yl)phenyl)-3-(trimethylsilyl)prop-2-} yn-1-ol (S4)

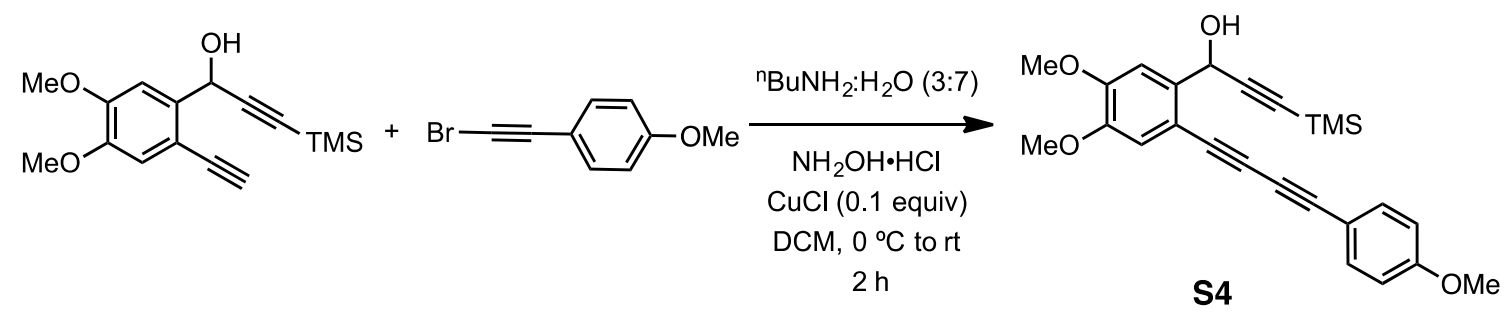

Following general procedure A: 1-(2-Ethynyl-4,5-dimethoxyphenyl)-3-(trimethylsilyl)prop-2yn-1-ol (200 mg, 0.69 mmol), bromoethynyl-4-methoxybenzene ${ }^{6}$ (199 mg, 0.89 mmol), CuCl (7 $\mathrm{mg}, 0.069 \mathrm{mmol}), n$-butylamine $/ \mathrm{H}_{2} \mathrm{O}$ (v:v, 30:70, $\left.10 \mathrm{~mL}\right), \mathrm{NH}_{2} \mathrm{OH} \bullet \mathrm{HCl}(\approx 20 \mathrm{mg})$, and DCM (10 $\mathrm{mL}$ ) were used to prepare the triyne $\mathbf{S 4}$. Purification of the crude material by flash chromatography (hexanes:EtOAc 4:1) provided triyne $\mathbf{S 4}$ (115 mg, $0.275 \mathrm{mmol}, 40 \%$ ) as a yellow oil.

${ }^{1}$ H NMR (400 MHz, $\left.\mathrm{CDCl}_{3}\right): \delta=7.46\left(\mathrm{nfod}, J_{a p p}=8.7 \mathrm{~Hz}, 2 \mathrm{H}\right), 7.26(\mathrm{~s}, 1 \mathrm{H}), 6.99(\mathrm{~s}, 1 \mathrm{H}), 6.86$ $\left(\right.$ nfod, $\left.J_{a p p}=8.8 \mathrm{~Hz}, 2 \mathrm{H}\right), 5.85(\mathrm{~s}, 1 \mathrm{H}, \mathrm{CHOH}), 3.93(\mathrm{~s}, 3 \mathrm{H}, \mathrm{CH} 3 \mathrm{O}), 3.88\left(\mathrm{~s}, 3 \mathrm{H}, \mathrm{CH}_{3} \mathrm{O}^{\prime}\right), 3.83(\mathrm{~s}$, $\left.3 \mathrm{H}, \mathrm{CH}_{3} \mathrm{O}^{\prime \prime}\right), 2.47$ (br s, $\left.1 \mathrm{H}, \mathrm{OH}\right)$, and 0.21 (s, 9H, $\left.\mathrm{Si}\left(\mathrm{CH}_{3}\right)_{3}\right)$.

${ }^{13} \mathrm{C}$ NMR (100 MHz, $\left.\mathrm{CDCl}_{3}\right): \delta=160.5,150.4,148.8,137.4,134.2,115.3,114.3,113.8,112.7$, $110.2,104.6,91.9,83.0,78.4,78.1,72.8,63.3,56.2,56.1,55.5$, and -0.0 .

IR (neat): 3488, 3003, 2958, 2936, 2903, 2837, 2211, 2173, 1601, 1406, 1210, 1029, and $666 \mathrm{~cm}^{-1}$. HRMS (ESI) m/z: [M + Na] $]^{+}$Calcd for $\mathrm{C}_{25} \mathrm{H}_{26} \mathrm{NaO}_{4} \mathrm{Si} 441.1498$; Found 441.1489. 


\section{Methyl 4-((2-(1-Hydroxy-3-(trimethylsilyl)prop-2-yn-1-yl)-4,5-dimethoxyphenyl)buta-1,3- diyn-1-yl)benzoate (S5)}

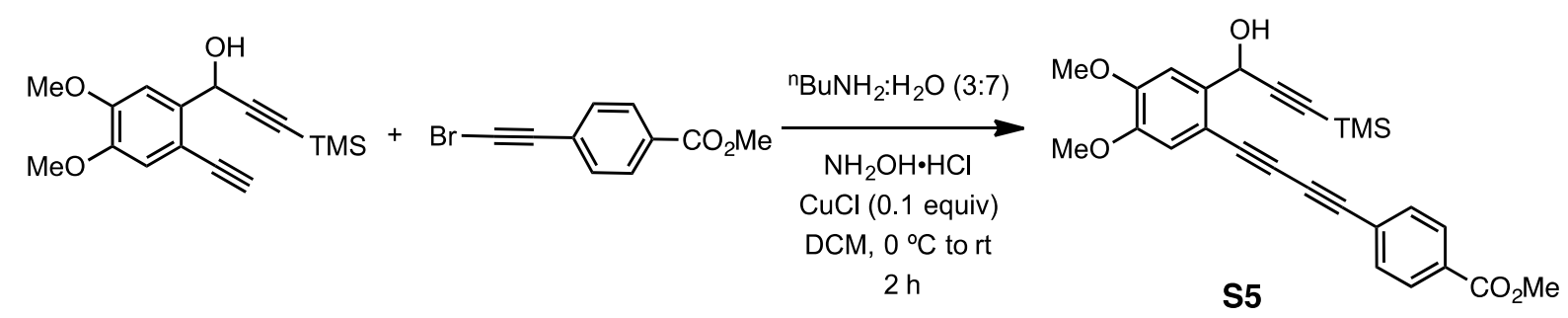

Following general procedure A: 1-(2-Ethynyl-4,5-dimethoxyphenyl)-3-(trimethylsilyl)-prop-2yn-1-ol (200 mg, $0.69 \mathrm{mmol}$ ) and methyl 4-(bromoethynyl)benzoate ${ }^{7}$ (214 mg, $\left.0.89 \mathrm{mmol}\right), \mathrm{CuCl}$ (9 mg, $0.089 \mathrm{mmol}), n$-butylamine/ $\mathrm{H}_{2} \mathrm{O}(\mathrm{v}: \mathrm{v}, 30: 70,10 \mathrm{~mL})$, and DCM (10 mL) were used to prepare triyne S5. Purification of the crude material by flash chromatography (hexanes:EtOAc 4:1) provided triyne $\mathbf{S 5}$ (160 $\mathrm{mg}, 0.358 \mathrm{mmol}, 52 \%)$ as a yellow oil.

${ }^{1} \mathbf{H}$ NMR (400 MHz, $\left.\mathrm{CDCl}_{3}\right): \delta=7.91\left(\mathrm{nfod}, J_{a p p}=8.5 \mathrm{~Hz}, 2 \mathrm{H}, \mathrm{ArH} 22 \& H 20\right), 7.47$ (nfod, $J_{a p p}$ $=8.4 \mathrm{~Hz}, 2 \mathrm{H}, \operatorname{ArH} 19 \& H 23), 7.17(\mathrm{~s}, 1 \mathrm{H}, H 6), 6.90(\mathrm{~s}, 1 \mathrm{H}, H 3), 5.73(\mathrm{~d}, J=4.5 \mathrm{~Hz}, 1 \mathrm{H}$, $\mathrm{ArCHOH}$ ), 3.84 (s, 3H, $\mathrm{CH}_{3} \mathrm{O}$ ), 3.82 (s, 3H, CH3 $\mathrm{O}^{\prime}$ ), 3.79 (s, 3H, CH3 $\mathrm{O}^{\prime}$ '), 2.38 (br s, 1H, $\mathrm{OH}$ ), and $0.10\left(\mathrm{~s}, 9 \mathrm{H}, \mathrm{Si}\left(\mathrm{CH}_{3}\right)_{3}\right)$.

${ }^{13} \mathrm{C}$ NMR (100 MHz, $\left.\mathrm{CDCl}_{3}\right): \delta=166.3,150.7,148.7,137.6,132.3,130.3,129.6,126.5,115.2$, $111.9,110.0,104.4,91.9,81.5,80.4,76.8,63.1,56.1,56.0,52.4$, and -0.2 .

IR (neat): 3487, 3002, 2954, 2902, 2856, 2837, 1721, 1601, 1350, 1271, 1210, 1175, 1015, 990 and $842 \mathrm{~cm}^{-1}$.

HRMS (ESI) m/z: [M + Na $]^{+}$Calcd for $\mathrm{C}_{26} \mathrm{H}_{26} \mathrm{O}_{5} \mathrm{NaSi} 469.1442$; Found 469.1441. 


\section{1,12-Dimethoxy-7-methyl-8-(trimethylsilyl)-9H-indeno[2,1-c]phenanthren-9-one (16a-syn)}

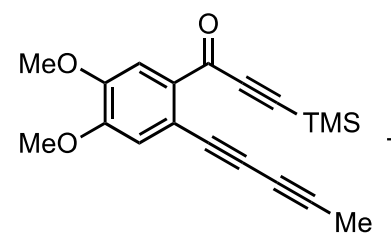

13

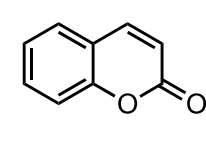

$7 a$

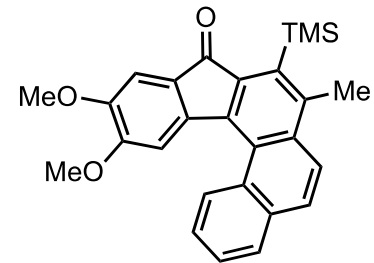

16a-syn

Following general procedure $\mathbf{C}$ : Triyne $\mathbf{1 3}^{8}(30 \mathrm{mg}, 0.09 \mathrm{mmol})$ and $2 \mathrm{H}$-chromen-2-one (7a, 40 $\mathrm{mg}, 0.27 \mathrm{mmol})$ were dissolved in $(3 \mathrm{~mL})$ of $\mathrm{CHCl}_{3}$ and heated at $85{ }^{\circ} \mathrm{C}$ for $14 \mathrm{~h}$. Solvent removal gave the crude material that was purified by MPLC (hexanes:EtOAc, 9:1) to provide 16a-syn (14.5 $\mathrm{mg}, 0.034 \mathrm{mmol}, 38 \%$ ) as a red crystalline solid.

${ }^{1} \mathbf{H}$ NMR (400 MHz, $\left.\mathrm{CDCl}_{3}\right): \delta=8.89(\mathrm{dd}, J=8.3,0.9 \mathrm{~Hz}, 1 \mathrm{H}, H 1), 7.89(\mathrm{~d}, J=9.2 \mathrm{~Hz}, 1 \mathrm{H}$, H5), $7.86(\mathrm{dd}, J=8.0,1.4 \mathrm{~Hz}, 1 \mathrm{H}, H 4), 7.76$ (d, $J=9.1 \mathrm{~Hz}, 1 \mathrm{H}, H 6), 7.60$ (ddd, $J=8.2,7.0,1.3$ Hz, 1H, H2 or H3), 7.59 (s, 1H, H13), 7.49 (ddd, J=8.3, 6.9, 1.4 Hz, 1H, H2 or H3), 7.16 (s, $1 \mathrm{H}, \mathrm{H} 10$ ), 3.93 (s, 3H, $\left.\mathrm{CH}_{3} \mathrm{O}\right), 3.82$ (s, 3H, $\mathrm{CH}_{3} \mathrm{O}$ ') 2.79 (s, 3H, $\left.\mathrm{ArCH}_{3}\right)$, and $0.48(\mathrm{~s}, 9 \mathrm{H}$, $\left.\mathrm{Si}\left(\mathrm{CH}_{3}\right)_{3}\right)$.

${ }^{13}$ C NMR (100 MHz, $\left.\mathrm{CDCl}_{3}\right): \delta=194.9,153.0,149.2,142.2,140.9,139.9,138.3,137.3,135.5$, 133.3, 129.7, 129.0, 128.9, 128.1, 128.0, 127.7, 126.7, 124.3, 123.1, 107.6, 107.0, 56.323, 56.316, 21.6, and 2.6.

IR (neat): 3003, 2951, 2926, 2870, 2853, 2835, 1700, 1214, 989, 839, and $701 \mathrm{~cm}^{-1}$. HRMS (ESI) m/z: $[\mathrm{M}+\mathrm{H}]^{+}$Calcd for $\mathrm{C}_{27} \mathrm{H}_{27} \mathrm{O}_{3} \mathrm{Si}$ 427.1729; Found 427.1707. mp: $138-140{ }^{\circ} \mathrm{C}$. 


\section{1,12-Dimethoxy-7-methyl-8-(trimethylsilyl)-9H-indeno[2,1-c]phenanthren-9-one (16a-syn)} and

9,10-Dimethoxy-5-methyl-6-(trimethylsilyl)-7H-indeno[1,2-a]phenanthren-7-one (16a-anti)

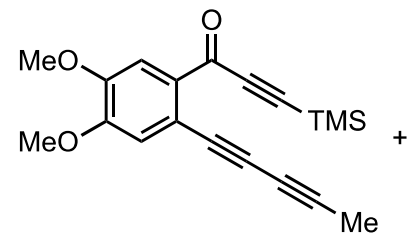

13

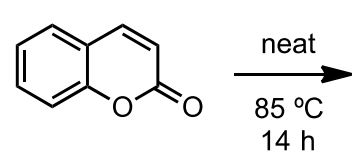

$7 a$

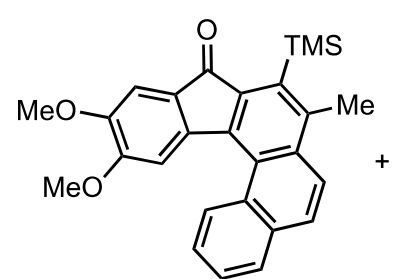

16a-syn

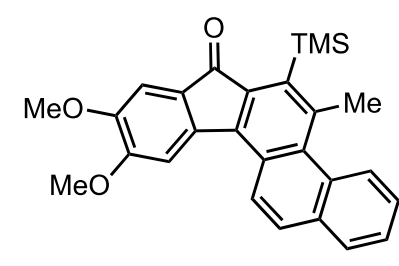

16a-anti

A mixture of triyne 13 (164 mg, $0.5 \mathrm{mmol})$ and 2H-chromen-2-one (7a, $739 \mathrm{mg}, 5.0 \mathrm{mmol})$ were combined in a screw-capped culture tube and heated at $85{ }^{\circ} \mathrm{C}$ for $14 \mathrm{~h}$. The crude mixture was dissolved in $10 \mathrm{~mL}$ of MeOH: $\mathrm{H} 2 \mathrm{O}$ (1:1) containing $\mathrm{KOH}$ (140 mg, 5 equiv) and this mixture was stirred for $4 \mathrm{~h}$ at room temperature. Disappearance of the excess coumarin was judged by TLC and the crude mixture was extracted with DCM and concentrated. Purification of the crude material by MPLC (hexanes:EtOAc, 9:1) provided, in order of elution, 16a-syn (60 mg, $0.014 \mathrm{mmol}, 28 \%$ ) as a red crystalline solid and 16a-anti (10 $\mathrm{mg}, 0.023,5 \%)$ as an orange crystalline solid.

${ }^{1} \mathbf{H}$ NMR (400 MHz, $\left.\mathrm{CDCl}_{3}\right): \delta=8.56(\mathrm{dd}, J=7.4,1.8 \mathrm{~Hz}, 1 \mathrm{H}, H 4), 8.19$ (d, $J=9.1 \mathrm{~Hz}, 1 \mathrm{H}$, H12), 7.86 (dd, $J=7.3,2.1 \mathrm{~Hz}, 1 \mathrm{H}, H 1), 7.75$ (d, $J=9.1 \mathrm{~Hz}, 1 \mathrm{H}, H 13), 7.61$ (ddd, $J=7.1,7.1$, $1.5 \mathrm{~Hz}, 1 \mathrm{H}, H 2$ or $H 3), 7.57$ (ddd, $J=7.1,7.1,1.9 \mathrm{~Hz}, 1 \mathrm{H}, H 2$ or H3), 7.52 (s, $1 \mathrm{H}, H 11), 7.23$ (s, $1 \mathrm{H}, \mathrm{H8}), 4.11\left(\mathrm{~s}, 3 \mathrm{H}, \mathrm{CH}_{3} \mathrm{O}\right), 3.95$ (s, 3H, CH $\left.3 \mathrm{O}^{\prime}\right), 3.06$ (s, 3H, $\left.\mathrm{ArCH}_{3}\right)$, and $0.54(\mathrm{~s}, 9 \mathrm{H}$, $\left.\mathrm{Si}\left(\mathrm{CH}_{3}\right)_{3}\right)$.

${ }^{13} \mathrm{C}$ NMR (100 MHz, $\left.\mathrm{CDCl}_{3}\right): \delta=195.1,153.8,149.2,143.3,140.8,139.8,139.3,137.8,136.1$, $134.1,130.8,129.5,129.0,128.7,128.4,127.9,127.3,125.7,121.8,107.5,107.3,56.7,56.3,29.8$ and 3.1 .

IR (neat): 3055, 2984, 2954, 2913, 2845, 1717, 1598, 1475, 1459, 1344, 1295, 1254, 1197, 1072, 1018,849 and $739 \mathrm{~cm}^{-1}$.

HRMS (ESI) m/z: [M + Na] ${ }^{+}$Calcd for $\mathrm{C}_{27} \mathrm{H}_{26} \mathrm{NaO}_{3} \mathrm{Si} 449.1549$; Found 449.1585. mp: $228-230{ }^{\circ} \mathrm{C}$. 


\section{1,12-Dimethoxy-7-methyl-9-oxo-8-(trimethylsilyl)-9H-indeno[2,1-c]phenanthren-2-yl Acetate (16b)}

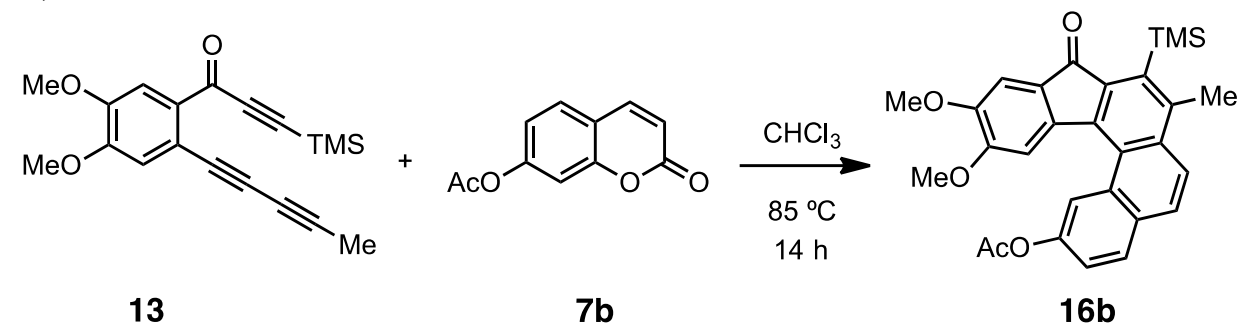

Following general procedure C: Triyne 13 (50 mg, $0.154 \mathrm{mmol})$ and 2-oxo-2H-chromen-7-yl acetate ${ }^{9}(7 \mathbf{b}, 94 \mathrm{mg}, 0.46 \mathrm{mmol})$ were dissolved in $\mathrm{CHCl}_{3}(5 \mathrm{~mL})$ and used to prepare the phenanthrene derivative 16b. Purification of the crude material by MPLC (hexanes:EtOAc, 9:1) provided the phenanthrene derivative $16 \mathbf{b}(25 \mathrm{mg}, 0.05 \mathrm{mmol}, 34 \%)$ as a red crystalline solid.

${ }^{1} \mathbf{H}$ NMR (400 MHz, $\left.\mathrm{CDCl}_{3}\right): \delta 8.52(\mathrm{ddd}, J=2.3,0.5,0.5 \mathrm{~Hz}, 1 \mathrm{H}, H 1), 7.886(\mathrm{~d}, J=9.2 \mathrm{~Hz}$, 1H, H6), 7.883 (br d, $J=8.6 \mathrm{~Hz}, 1 \mathrm{H}, H 4), 7.75$ (dd, $J=9.4,0.5 \mathrm{~Hz}, 1 \mathrm{H}, H 5), 7.50$ (s, 1H, H13), $7.34(\mathrm{dd}, J=8.7,2.4 \mathrm{~Hz}, 1 \mathrm{H}, H 3), 7.17$ (s, 1H, H10), 3.93 (s, 3H, CH3 O), 3.84 (s, 3H, CH3O'), 2.78 (s, 3H, Ar- $\left.\mathrm{CH}_{3}\right), 2.3\left(\mathrm{~s}, 3 \mathrm{H}, \mathrm{CH}_{3}\right)$, and 0.47 (s, 9H, $\left.\mathrm{Si}\left(\mathrm{CH}_{3}\right)_{3}\right)$.

${ }^{13}$ C NMR (100 MHz, $\left.\mathrm{CDCl}_{3}\right): \delta$ 194.7, 169.7, 153.3, 149.3, 147.5, 142.3, 140.5, 140.0, 138.4, 137.7, 135.5, 131.2, 130.7, 129.3, 128.4, 127.2, 126.7, 123.0, 122.4, 120.4, 107.4, 107.0, 56.4, $56.3,21.7,21.2$, and $2.6 \mathrm{ppm}$.

IR (neat): 3057, 3001, 2946, 2900, 2836, 1759, 1700, 1364, 1310, 1270, 1246, 1205, 1183, and $735 \mathrm{~cm}^{-1}$.

HRMS (ESI) m/z: [M + H] $]^{+}$Calcd for $\mathrm{r}_{29} \mathrm{H}_{29} \mathrm{O}_{5} \mathrm{Si} 485.1784$; Found 485.1749. mp: $130-132{ }^{\circ} \mathrm{C}$. 


\section{1,12-Dimethoxy-7-methyl-9-oxo-8-(trimethylsilyl)-9H-indeno[2,1-c]phenanthren-2-yl} Ethyl Carbonate (16c)

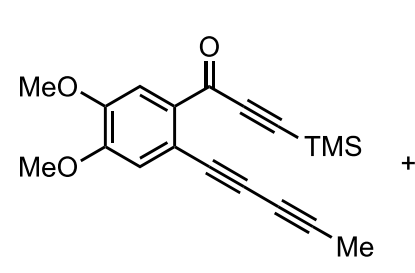

13

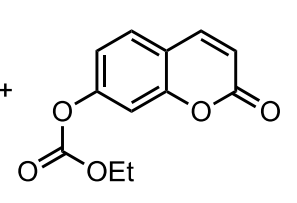

7c

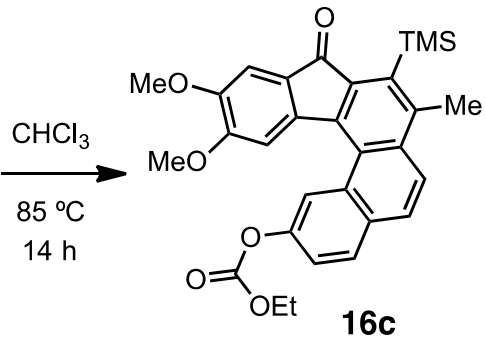

$16 c$

Following general procedure C: Triyne 13 (30 mg, $0.09 \mathrm{mmol})$ and ethyl 2-oxo-2H-chromene7-carboxylate ${ }^{10}(7 \mathbf{c}, 58 \mathrm{mg}, 0.27 \mathrm{mmol})$ were dissolved in $\mathrm{CHCl}_{3}(5 \mathrm{~mL})$ and used to prepare the phenanthrene derivative 16c. Purification of the crude material by MPLC (hexanes:EtOAc, 9:1) provided 16c (16 $\mathrm{mg}, 0.03 \mathrm{mmol}, 34 \%)$ as a red crystalline solid.

${ }^{1} \mathbf{H}$ NMR (400 MHz, $\left.\mathrm{CDCl}_{3}\right): \delta 8.62(\mathrm{ddd}, J=2.3,0.5,0.5 \mathrm{~Hz}, 1 \mathrm{H}, H 1), 7.90(\mathrm{~d}, J=9.3 \mathrm{~Hz}, 1 \mathrm{H}$, H6), 7.89 (dd, $J=8.6,0.5 \mathrm{~Hz}, 1 \mathrm{H}, \mathrm{H} 4), 7.75$ (dd, $J=9.5,0.6 \mathrm{~Hz}, 1 \mathrm{H}, H 5), 7.50$ (s, 1H, H13), $7.44(\mathrm{dd}, J=8.7,2.4 \mathrm{~Hz}, 1 \mathrm{H}, H 3), 7.17$ (s, 1H, H10), 4.31 (q, $\left.J=7.2 \mathrm{~Hz}, 2 \mathrm{H}, \mathrm{OCH}_{2}\right), 3.94$ (s, $\left.3 \mathrm{H}, \mathrm{CH}_{3} \mathrm{O}\right), 3.85$ (s, 3H, $\left.\mathrm{CH}_{3} \mathrm{O}^{\prime}\right), 2.78\left(\mathrm{~s}, 3 \mathrm{H}, \mathrm{Ar}-\mathrm{CH}_{3}\right), 1.39$ (t, J=7.2 Hz, 3H, CH3), and 0.47 (s, 9H, $\left.\mathrm{Si}\left(\mathrm{CH}_{3}\right)_{3}\right)$.

${ }^{13}$ C NMR (100 MHz, $\left.\mathrm{CDCl}_{3}\right): \delta$ 194.7, 154.0, 153.3, 149.3, 147.9, 142.3, 140.5, 140.0, 138.4, $137.8,135.5,131.3,130.6,129.5,128.3,127.2$, 126.6, 123.2, 121.9, 120.0, 107.5, 107.1, 65.3, $56.4,56.3,21.7,14.4$, and 2.6.

IR (neat): 3054, 2943, 2901, 2836, 1756, 1700, 1065, and $735 \mathrm{~cm}^{-1}$.

HRMS (ESI) m/z: [M + H] ${ }^{+}$Calcd for $\mathrm{C}_{30} \mathrm{H}_{31} \mathrm{O}_{6} \mathrm{Si}$ 515.1885; Found 515.1870.

mp: $182-184^{\circ} \mathrm{C}$. 


\section{2,11,12-Trimethoxy-7-methyl-8-(trimethylsilyl)-9H-indeno[2,1-c]phenanthren-9-one (16d)}

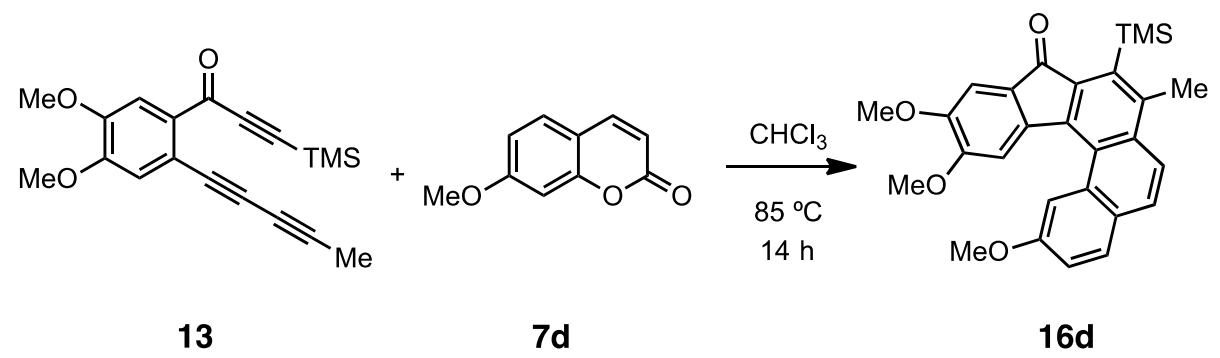

Following general procedure C: Triyne 13 (50 mg, 0.154 mmol) and 7-methoxy-2H-chromen2-one ${ }^{11}$ in $(\mathbf{7 d}, 81 \mathrm{mg}, 0.46 \mathrm{mmol})$ were dissolved in $\mathrm{CHCl}_{3}(5 \mathrm{~mL})$ and used to prepare the phenanthrene derivative 16d Purification of the crude material by MPLC (hexanes:EtOAc, 9:1) provided 16d (24 mg, $0.052 \mathrm{mmol}, 34 \%$ ) as a red crystalline solid.

${ }^{1} \mathbf{H}$ NMR (400 MHz, $\left.\mathrm{CDCl}_{3}\right): \delta=8.24(\mathrm{dd}, J=2.5,0.5 \mathrm{~Hz}, 1 \mathrm{H}, H 1), 7.79$ (d, $J=8.7 \mathrm{~Hz}, 1 \mathrm{H}$, H4), 7.77 (d, $J=9.2 \mathrm{~Hz}, 1 \mathrm{H}, H 6), 7.70$ (br d, $J=9.21 \mathrm{H}, H 5) 7.60$ (s, 1H, H13), 7.25 (dd, $J=8.7$, $2.5 \mathrm{~Hz}, 1 \mathrm{H}, \mathrm{H3}), 7.16$ (s, 1H, H1O), 3.93 (s, 3H, $\left.\mathrm{CH}_{3} \mathrm{O}\right), 3.89$ (s, 3H, $\mathrm{CH}_{3} \mathrm{O}$ '), 3.82 (s, 3H, $\mathrm{CH}_{3} \mathrm{O}$ '’), 2.77 (s, 3H, Ar-Me), and 0.48 (s, 9H, $\left.\mathrm{Si}\left(\mathrm{CH}_{3}\right)_{3}\right)$.

${ }^{13}$ C NMR (100 MHz, $\left.\mathrm{CDCl}_{3}\right): \delta$ 194.8, 156.5, 152.8, 149.2, 142.1, 140.8, 140.0, 137.9, 137.2, $135.9,131.1,129.5,128.7,127.9,127.2$, 127.0, 120.8, 117.6, 111.3, 107.9, 107.0, 56.3, 56.3, $55.8,21.7$, and 2.6.

IR (neat): 3004, 2955, 2927, 2853, 2837, 1701, 1388, 1215, and $768 \mathrm{~cm}^{-1}$.

HRMS (ESI) m/z: [M + Na] ${ }^{+}$Calcd for $\mathrm{C}_{28} \mathrm{H}_{29} \mathrm{O}_{4} \mathrm{Na} \mathrm{Si} 479.1649$; Found 479.1644. mp: $262-264{ }^{\circ} \mathrm{C}$. 


\section{1,12-Dimethoxy-7-methyl-2-(prop-2-yn-1-yloxy)-8-(trimethylsilyl)-9H-indeno[2,1-} c]phenanthren-9-one (16e)

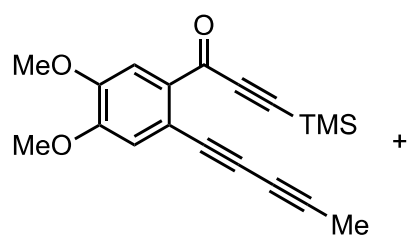

13

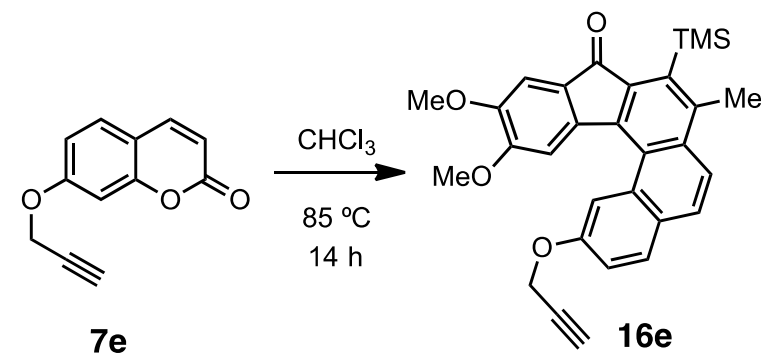

Following general procedure C: Triyne 13 (50 mg, 0.154 mmol) and 7-(prop-2-yn-1-yloxy)-2Hchromen-2-one ${ }^{12}(7 \mathrm{e}, 92 \mathrm{mg}, 0.462 \mathrm{mmol})$ were dissolved in $\mathrm{CHCl}_{3}(5 \mathrm{~mL})$ and used to prepare the phenanthrene derivative 16e. Purification of the crude material by MPLC (hexanes:EtOAc, 9:1) provided 16e (25 mg, $0.05 \mathrm{mmol}, 36 \%)$ as a red crystalline solid.

${ }^{1} \mathbf{H}$ NMR (400 MHz, $\left.\mathrm{CDCl}_{3}\right): \delta 8.34(\mathrm{ddd}, J=2.5,0.6,0.6 \mathrm{~Hz}, 1 \mathrm{H}, H 1), 7.82(\mathrm{~d}, J=8.7 \mathrm{~Hz}, 1 \mathrm{H}$, H4), 7.78 (d, $J=9.2 \mathrm{~Hz}, 1 \mathrm{H}, \mathrm{H} 3), 7.70$ (br d, $J=9.3 \mathrm{~Hz}, 1 \mathrm{H}, H 5), 7.57$ (s, 1H, H13), 7.32 (dd, $J$ = 8.7, $2.5 \mathrm{~Hz}, 1 \mathrm{H}, H 3), 7.16(\mathrm{~s}, 1 \mathrm{H}, H 10), 4.77$ (d, $\left.J=2.4 \mathrm{~Hz}, 2 \mathrm{H}, \mathrm{OCH}_{2}\right), 3.93(\mathrm{~s}, 3 \mathrm{H}, \mathrm{CH} 3 \mathrm{O})$, $3.84\left(\mathrm{~s}, 3 \mathrm{H}, \mathrm{CH}_{3} \mathrm{O}^{\prime}\right), 2.78(\mathrm{~s}, 3 \mathrm{H}, \mathrm{Ar}-\mathrm{Me}), 2.49(\mathrm{t}, J=2.3 \mathrm{~Hz}, 1 \mathrm{H}, \equiv \mathrm{CH})$, and $0.48\left(\mathrm{~s}, 9 \mathrm{H}, \mathrm{Si}\left(\mathrm{CH}_{3}\right) 3\right)$. ${ }^{13}$ C NMR (100 MHz, $\left.\mathrm{CDCl}_{3}\right): \delta$ 194.8, 154.5, 152.9, 149.1, 142.0, 140.6, 140.0, 138.1, 137.4, 135.8, 131.0, 129.4, 128.5, 128.4, 127.1, 126.8, 121.2, 117.1, 113.6, 107.8, 106.9, 78.4, 76.0, $56.4,56.38,56.31,31.0,21.6$, and 2.63.

IR (neat): 3289, 3257, 3049, 3002, 2950, 2903, 2835, 1698, 1601, 1201, 952 and $766 \mathrm{~cm}^{-1}$. HRMS (ESI) m/z: [M + Na $]^{+}$Calcd for $\mathrm{C}_{30} \mathrm{H}_{28} \mathrm{NaO}_{4} \mathrm{Si}$ 503.1655; Found 503.1641. mp: $148-150{ }^{\circ} \mathrm{C}$. 


\section{9,10-Dimethoxy-5-methyl-6-(trimethylsilyl)-1H-1,4-epoxybenzo[c]fluoren-7(4H)-one (17)}

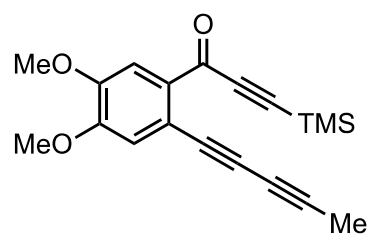

13

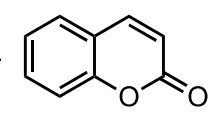

$7 \mathbf{a}$

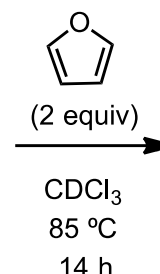

$14 \mathrm{~h}$

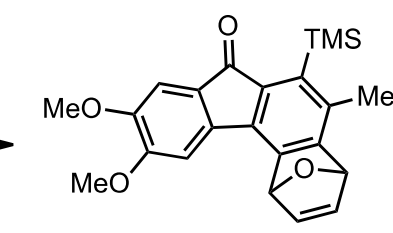

17

Triyne 13 (10 mg, $0.03 \mathrm{mmol})$, coumarin (7a, $45 \mathrm{mg}, 0.3 \mathrm{mmol})$, and furan (4 mg, $0.06 \mathrm{mmol})$ were dissolved in $\mathrm{CDCl}_{3}(0.6 \mathrm{~mL})$ and heated at $85^{\circ} \mathrm{C}$ for $14 \mathrm{~h}$. Following concentration the residue was purified by MPLC (hexanes:EtOAc, 4:1) to provide the fluorenone derivative 17 (10 $\mathrm{mg}, 0.025 \mathrm{~mol}, 85 \%$ ) as a yellow crystalline solid. A proton NMR spectrum of the crude product mixture obtained upon solvent removal is provided on page S96. Analysis indicates a 95:5 ratio of products 17 and 16a-syn.

${ }^{1}$ H NMR (400 MHz, $\left.\mathrm{CDCl}_{3}\right): \delta=7.17(\mathrm{~s}, 1 \mathrm{H}, H 8), 7.043$ (ddd, $J=4.8,1.0,1.0 \mathrm{~Hz}, 1 \mathrm{H}, H 2$ or H3), 7.042 (ddd, $J=4.8,1.0,1.0 \mathrm{~Hz}, 1 \mathrm{H}, H 2$ or H3), 6.91 (s, 1H, H11), 6.07 (ddd, $J=1.0,1.0$, $1.0 \mathrm{~Hz}, 1 \mathrm{H}$, bridgehead $H 1$ or $H 4), 5.81$ (ddd, $J=1.0,1.0,1.0 \mathrm{~Hz}, 1 \mathrm{H}$, bridgehead $H 1$ or $H 4$ ), 4.03 (s, 3H, $\left.\mathrm{CH}_{3} \mathrm{O}\right), 3.91$ (s, 3H, $\left.\mathrm{CH}_{3} \mathrm{O}^{\prime}\right), 2.38$ (s, 3H, $\mathrm{ArCH}$ ), and 0.39 (s, 9H, $\left.\mathrm{Si}\left(\mathrm{CH}_{3}\right)_{3}\right)$.

${ }^{13} \mathrm{C}$ NMR (100 MHz, $\left.\mathrm{CDCl}_{3}\right): \delta=193.5,154.4,151.9,149.7,142.0,141.5,140.8,140.6,139.2$, 138.0, 135.0, 133.4, 127.9, 107.0, 104.3, 81.1, 80.7, 56.5, 56.3, 20.8, and 2.9.

IR (neat): 3064, 2994, 2916, 2853, 1656, 1620, 1479, 1467, 1372, 1331, 1281, 1229, 1121, 1085, $1055,873,758$ and $715 \mathrm{~cm}^{-1}$.

HRMS (ESI) m/z: [M + H] ${ }^{+}$Calcd for $\mathrm{C}_{23} \mathrm{H}_{25} \mathrm{O}_{4} \mathrm{Si}$ 393.1522; Found 393.1501. mp: $174-176^{\circ} \mathrm{C}$. 


\section{1-(2-((4-Fluorophenyl)buta-1,3-diyn-1-yl)-4,5-dimethoxyphenyl)-3-(trimethylsilyl)prop-2-} yn-1-one (18a)
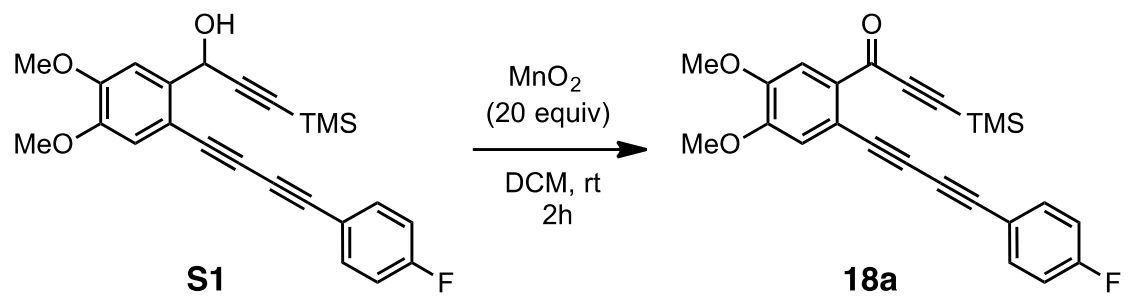

Following general procedure B: Activated manganese dioxide ( $839 \mathrm{mg}, 9.65 \mathrm{mmol}, 20$ equiv) was added to a stirred solution of triyne $\mathbf{S 1}(195 \mathrm{mg}, 0.482 \mathrm{mmol})$ in DCM (10 mL). After stirring for 2 hours, the reaction mixture was filtered by passing through a Celite ${ }^{\circ}$ plug. The filtrate was concentrated to provide ketone $\mathbf{1 8 a}(160 \mathrm{mg}, 0.396 \mathrm{mmol}, 82 \%)$ as a brown crystalline solid.

${ }^{1} \mathbf{H}$ NMR $\left(400 \mathrm{MHz}, \mathrm{CDCl}_{3}\right): \delta=7.62(\mathrm{~s}, 1 \mathrm{H}, H 6), 7.51\left(\mathrm{app} \mathrm{dd}, 2 \mathrm{H}, J=9.0_{\mathrm{HH}}, 5.3_{\mathrm{HF}} \mathrm{Hz}\right.$, ArH19\& ArH23), 7.07 (s, 1H, H3), 7.03 (app dd, $J=9.1_{\mathrm{HH}}, 8.4 \mathrm{HF} \mathrm{Hz,} \mathrm{2H,} \mathrm{ArH2O} \mathrm{\&} \mathrm{ArH22),}$ 3.95 (s, 6H, CH3O, $\left.\mathrm{CH}_{3} \mathrm{O}^{\prime}\right)$, and 0.32 (s, 9H, $\left.\mathrm{Si}\left(\mathrm{CH}_{3}\right)_{3}\right)$.

${ }^{13} \mathrm{C}$ NMR (100 MHz, $\left.\mathrm{CDCl}_{3}\right): \delta=175.1,163.2\left(\mathrm{~d}, \mathrm{C}-\mathrm{F},{ }^{1} \mathrm{~J}=251 \mathrm{~Hz}\right), 152.6,149.5,134.7$ (d, C$\left.\mathrm{F},{ }^{3} J=8.4 \mathrm{~Hz}\right), 132.7,118.1\left(\mathrm{~d}, \mathrm{C}-\mathrm{F},{ }^{4} J=3.7 \mathrm{~Hz}\right), 117.2,116.1\left(\mathrm{~d}, \mathrm{C}-\mathrm{F},{ }^{2} J=22.2 \mathrm{~Hz}\right), 115.8$, $113.7,101.8,101.6,82.4,80.0,79.2,74.5,56.5,56.2$, and -0.5 .

IR (neat): 3074, 3007, 2959, 2937, 2903, 2850, 2835, 2210, 2149, 1703, 1398, 1355, 1319, 1266, 1250, 1202, 1147, and $706 \mathrm{~cm}^{-1}$.

HRMS (ESI) m/z: [M + Na $]^{+}$Calcd for $\mathrm{C}_{24} \mathrm{H}_{21} \mathrm{FNaO}_{3} \mathrm{Si}$ 427.1142; Found 427.1191.

mp: $142-144^{\circ} \mathrm{C}$. 


\section{4-((4,5-Dimethoxy-2-(3-(trimethylsilyl)propioloyl)phenyl)buta-1,3-diyn-1-yl)benzonitrile} (18b)
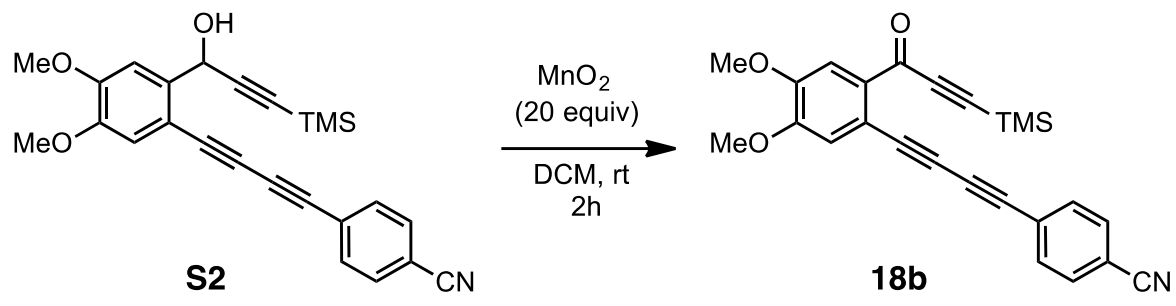

Following general procedure B: Activated manganese dioxide ( $842 \mathrm{mg}, 9.68 \mathrm{mmol}, 20$ equiv) was added to a stirred solution of the triyne S2 $(200 \mathrm{mg}, 0.484 \mathrm{mmol})$ in DCM $(10 \mathrm{~mL})$. After stirring for 2 hours, the reaction mixture was filtered by passing through a Celite ${ }^{\circ}$ plug. The filtrate was concentrated to provide ketone $\mathbf{1 8 b}(130 \mathrm{mg}, 0.316 \mathrm{mmol}, 65 \%)$ as a yellow crystalline solid. ${ }^{1} \mathbf{H}$ NMR $\left(400 \mathrm{MHz}, \mathrm{CDCl}_{3}\right): \delta=7.66(\mathrm{~s}, 1 \mathrm{H}, \mathrm{H6}), 7.63\left(\mathrm{nfod}, J_{a p p}=8.2 \mathrm{~Hz}, 2 \mathrm{H}, \mathrm{ArH19} / 23\right.$ or ArH20/22), 7.59 (nfod, $J_{a p p}=8.2 \mathrm{~Hz}, 2 \mathrm{H}, \mathrm{ArH19/23}$ or ArH2O/22), 7.09 (s, 1H, H3), 3.97 (s, 3H, $\left.\mathrm{CH}_{3} \mathrm{O}\right), 3.96$ (s, 3H, $\left.\mathrm{CH}_{3} \mathrm{O}^{\prime}\right)$, and 0.31 (s, 9H, $\left.\mathrm{Si}\left(\mathrm{CH}_{3}\right)_{3}\right)$.

${ }^{13} \mathrm{C}$ NMR $\left(100 \mathrm{MHz}, \mathrm{CDCl}_{3}\right): \delta=175.0,152.6,149.8,133.0,132.9,132.3,127.0,118.4,117.3$, $115.1,114.1,112.5,101.62,101.60,82.5,81.1,78.8,78.2,56.5,56.2$, and -0.5 .

IR (neat): 3088, 3062, 3007, 2960, 2939, 2903, 2851, 2837, 2227, 2207, 2150, 1517, 1356, 1269, 1205 , and $843 \mathrm{~cm}^{-1}$.

HRMS (ESI) m/z: [M + H] $]^{+}$Calcd for $\mathrm{C}_{25} \mathrm{H}_{22} \mathrm{NO}_{3} \mathrm{Si}$ 412.1369; Found 412.1362.

mp: $154-156{ }^{\circ} \mathrm{C}$. 


\section{1-(2-((4-Acetylphenyl)buta-1,3-diyn-1-yl)-4,5-dimethoxyphenyl)-3-(trimethylsilyl)prop-2- yn-1-one (18c)}
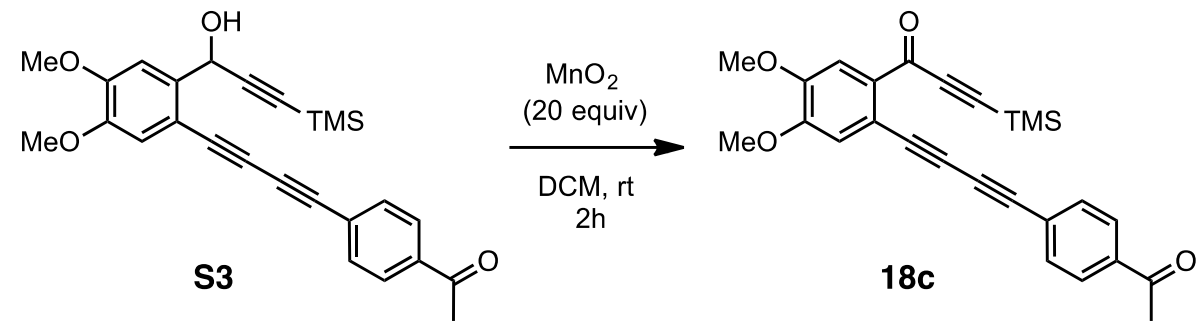

Following general procedure B: Activated manganese dioxide (549 mg, $6.32 \mathrm{mmol}, 20$ equiv) was added to a stirred solution of the triyne $\mathbf{S 3}(136 \mathrm{mg}, 0.316 \mathrm{mmol})$ in DCM $(10 \mathrm{~mL})$. After stirring for 2 hours, the reaction mixture was filtered by passing through a Celite ${ }^{\circledR}$ plug. The filtrate was concentrated to provide the diketone $18 \mathrm{c}(130 \mathrm{mg}, 0.303 \mathrm{mmol}, 96 \%)$ as a brown crystalline solid.

${ }^{1} \mathbf{H}$ NMR $\left(400 \mathrm{MHz}, \mathrm{CDCl}_{3}\right): \delta=7.92\left(\mathrm{~d}, J_{a p p}=8.4 \mathrm{~Hz}, 2 \mathrm{H}, \operatorname{Ar} H 20 \& \operatorname{Ar} H 22\right), 7.63(\mathrm{~s}, 1 \mathrm{H}, \mathrm{H} 6)$, 7.59 (d, Japp $=8.4 \mathrm{~Hz}, 2 \mathrm{H}, \operatorname{ArH} 19$ \& $\mathrm{ArH} 23), 7.08$ (s, 1H, H3), 3.96 (s, 3H, CH3O), 3.95 (s, 3H, $\left.\mathrm{CH}_{3} \mathrm{O}^{\prime}\right), 2.60$ (s, 3H, $\mathrm{COCH} 3$ ), and 0.31 (s, $\left.9 \mathrm{H}, \mathrm{Si}\left(\mathrm{CH}_{3}\right)_{3}\right)$.

${ }^{13}$ C NMR (100 MHz, $\left.\mathrm{CDCl}_{3}\right): \delta=197.2,175.0,152.6,149.7,137.0,132.8,132.7,128.4,126.8$, $117.2,115.4,113.9,101.69,101.66,82.3,81.7,78.8,77.7,56.5,56.2,26.8$, and -0.5 .

IR (neat): 3005, 2959, 2938, 2902, 2851, 2835, 2207, 2149, 1682, 1400, 1179, and $842 \mathrm{~cm}^{-1}$.

HRMS (ESI) m/z: $[\mathrm{M}+\mathrm{H}]^{+}$Calcd for $\mathrm{C}_{26} \mathrm{H}_{25} \mathrm{O}_{4} \mathrm{Si}$ 429.1522; Found 429.1517 . mp: $146-148{ }^{\circ} \mathrm{C}$. 
1-(4,5-Dimethoxy-2-((4-methoxyphenyl)buta-1,3-diyn-1-yl)phenyl)-3-(trimethylsilyl)prop-2yn-1-one (18d)
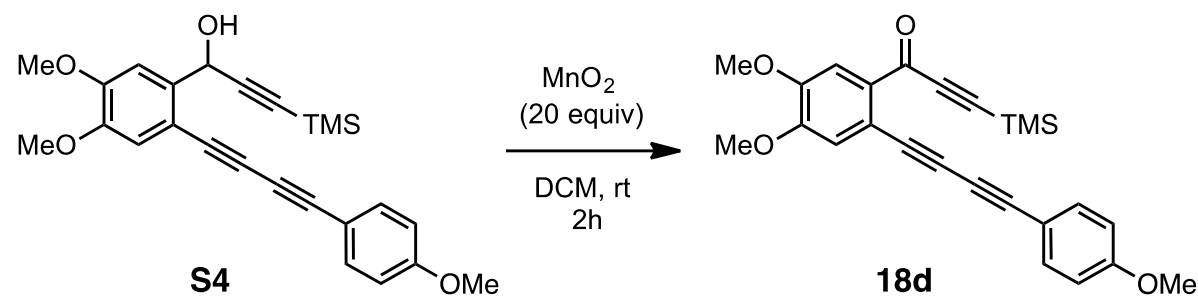

Following general procedure B: Activated manganese dioxide (457 mg, $5.26 \mathrm{mmol}, 20$ equiv) was added to a stirred solution of the triyne $\mathbf{S 4}(110 \mathrm{mg}, 0.263 \mathrm{mmol})$ in DCM (10 mL). After stirring for 2 hours, the reaction mixture was filtered by passing through a Celite ${ }^{\circ}$ plug. The filtrate was concentrated to provide ketone $\mathbf{1 8 d}(100 \mathrm{mg}, 0.240 \mathrm{mmol}, 91 \%)$ as a yellow crystalline solid. ${ }^{1} \mathbf{H}$ NMR (400 MHz, $\left.\mathrm{CDCl}_{3}\right): \delta=7.61$ (s, 1H, H6), $7.46\left(\right.$ nfod, $\left.J_{a p p}=8.9 \mathrm{~Hz}, 2 \mathrm{H}, H 19,23\right), 7.07$ (s, 1H, H3), 6.86 (nfod, $\left.J_{a p p}=8.7 \mathrm{~Hz}, 2 \mathrm{H}, \mathrm{H} 20,22\right), 3.95$ (s, 6H, $\mathrm{CH}_{3} \mathrm{O}, \mathrm{CH}_{3} \mathrm{O}$ '), 3.82 (s, 3H, $\left.\mathrm{CH}_{3} \mathrm{O}^{\prime \prime}\right)$, and $0.32\left(\mathrm{~s}, 9 \mathrm{H}, \mathrm{Si}\left(\mathrm{CH}_{3}\right)_{3}\right)$.

${ }^{13} \mathrm{C}$ NMR (100 MHz, $\left.\mathrm{CDCl}_{3}\right): \delta=175.2,160.6,152.6,149.4,134.3,132.5,117.1,116.4,114.3$, $113.9,113.5,101.9,101.7,83.9,79.9,79.5,73.6,56.5,56.2,55.5$, and -0.5 .

IR (neat): 3005, 2959, 2936, 2902, 2837, 2204, 2173, 2147, 1641, 1267, 1148, 1027, and 762 $\mathrm{cm}^{-1}$.

HRMS (ESI) m/z: [M + H] ${ }^{+}$Calcd for $\mathrm{C}_{25} \mathrm{H}_{25} \mathrm{O}_{4} \mathrm{Si}$ 417.1522; Found 417.1524. mp: $106-108{ }^{\circ} \mathrm{C}$. 


\section{Methyl 4-((4,5-Dimethoxy-2-(3-(trimethylsilyl)propioloyl)phenyl)buta-1,3-diyn-1- yl)benzoate (18e)}
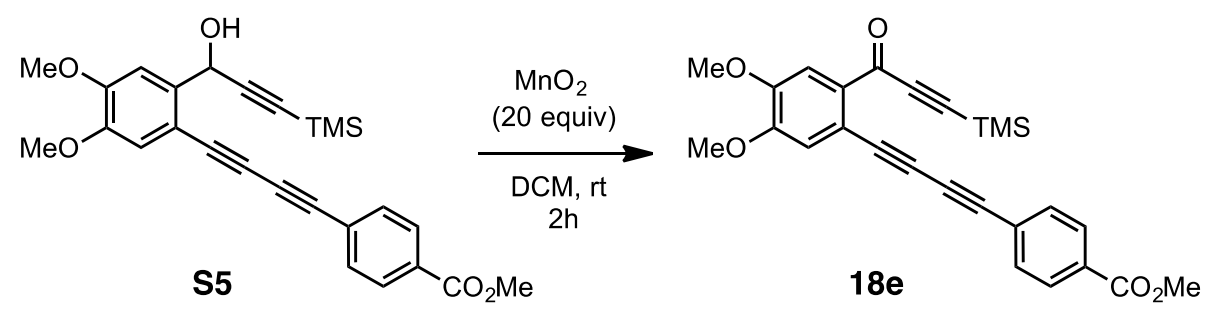

Following general procedure B: Activated manganese dioxide (585 mg, $6.72 \mathrm{mmol}, 20$ equiv) was added to a stirred solution of the triyne $\mathbf{S 5}(150 \mathrm{mg}, 0.336 \mathrm{mmol})$ in DCM $(10 \mathrm{~mL})$. After stirring for 2 hours, the reaction mixture was filtered by passing through a Celite ${ }^{\circ}$ plug. The filtrate was concentrated to provide ketone $\mathbf{1 8 e}(140 \mathrm{mg}, 0.315 \mathrm{mmol}, 94 \%)$ as a yellow crystalline solid. ${ }^{1} \mathbf{H}$ NMR (400 MHz, $\left.\mathrm{CDCl}_{3}\right): \delta=8.00\left(\right.$ nfod, $\left.J_{a p p}=8.2 \mathrm{~Hz}, 2 \mathrm{H}, \mathrm{ArH} 22 \& H 20\right), 7.63(\mathrm{~s}, 1 \mathrm{H}$, H6), 7.56 (nfod, $J_{a p p}=8.2 \mathrm{~Hz}, 2 \mathrm{H}, \mathrm{ArH} 19 \& H 23$ ), 7.08 (s, 1H, H3), 3.96 (s, 6H, $\mathrm{CH}_{3} \mathrm{O}, \mathrm{CH}_{3} \mathrm{O}^{\prime}$ ), 3.92 (s, 3H, $\left.\mathrm{CH}_{3} \mathrm{O}^{\prime \prime}\right)$, and $0.32\left(\mathrm{~s}, 9 \mathrm{H}, \mathrm{Si}\left(\mathrm{CH}_{3}\right)_{3}\right)$.

${ }^{13} \mathrm{C}$ NMR $\left(100 \mathrm{MHz}, \mathrm{CDCl}_{3}\right): \delta=175.0,166.4,152.6,149.6,132.7,132.5,130.4,129.7,126.6$, $117.2,115.5,113.8,101.7,101.6,82.3,81.5,78.8,77.5,56.5,56.1,52.4$, and -0.5 .

IR (neat): 3005, 2954, 2903, 2850, 2207, 2149, 1720, 1401, 1175, 1149, 1106, and $765 \mathrm{~cm}^{-1}$. HRMS (ESI) m/z: [M + Na] ${ }^{+}$Calcd for $\mathrm{C}_{26} \mathrm{H}_{25} \mathrm{O}_{5} \mathrm{NaSi} 467.1291$; Found 467.1278. mp: $122-124^{\circ} \mathrm{C}$. 
7-(4-Fluorophenyl)-11,12-dimethoxy-8-(trimethylsilyl)-9H-indeno[2,1-c]phenanthren-9-one (19a)

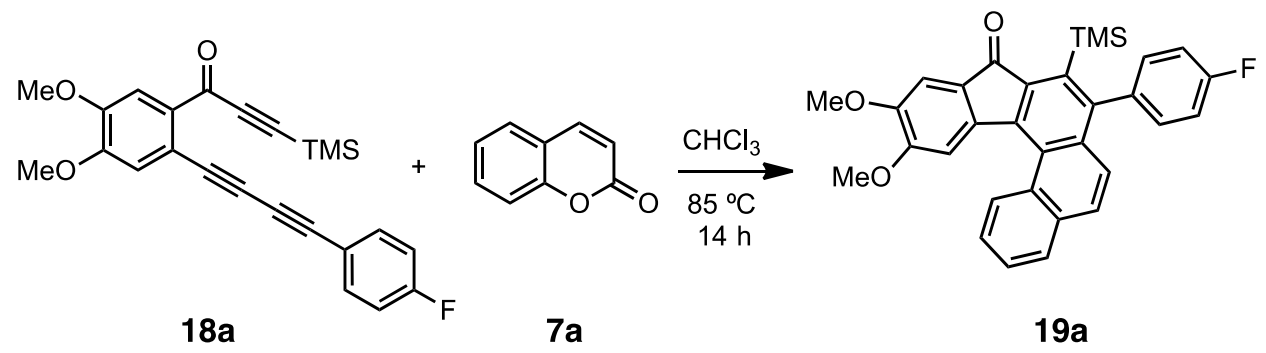

Following general procedure C: Triyne $\mathbf{1 8 a}(50 \mathrm{mg}, 0.123 \mathrm{mmol})$ and $2 \mathrm{H}$-chromen-2-one (7a, $54 \mathrm{mg}, 0.369 \mathrm{mmol})$ were dissolved in $(5 \mathrm{~mL})$ of $\mathrm{CHCl}_{3}$ to prepare the phenanthrene derivative 19a. Purification of the crude material by MPLC (hexanes: EtOAc, 9:1) provided 19a (20 mg, $0.039 \mathrm{mmol}, 32 \%$ ) as an orange crystalline solid.

${ }^{1} \mathbf{H}$ NMR (400 MHz, $\left.\mathrm{CDCl}_{3}\right): \delta=8.91(\mathrm{dd}, J=8.2,1.2 \mathrm{~Hz}, 1 \mathrm{H}, H 1), 7.83(\mathrm{dd}, J=7.8,1.6 \mathrm{~Hz}$, 1H, H4), 7.66 (s, $1 \mathrm{H}, H 13), 7.62$ (ddd, $J=8.1,7.0,1.3 \mathrm{~Hz}, 1 \mathrm{H}, H 2$ or H3), 7.56 (d, $J=9.0 \mathrm{~Hz}$, 1H, H5), $7.52(\mathrm{ddd}, J=8.4,7.0,1.6 \mathrm{~Hz}, 1 \mathrm{H}, \mathrm{H} 2$ or $H 3), 7.24-7.27$ (m, 3H, CH-CH=CF and H6), $7.20(\mathrm{~s}, 1 \mathrm{H}, \mathrm{H} 10), 7.17(\mathrm{dd}, J=8.7 \mathrm{~Hz}, 2 \mathrm{H}, \mathrm{HC}=\mathrm{CF}), 3.95\left(\mathrm{~s}, 3 \mathrm{H}, \mathrm{CH}_{3} \mathrm{O}\right), 3.84\left(\mathrm{~s}, 3 \mathrm{H}, \mathrm{CH}_{3} \mathrm{O}^{\prime}\right)$, and $0.02\left(\mathrm{~s}, 9 \mathrm{H}, \mathrm{Si}\left(\mathrm{CH}_{3}\right)_{3}\right)$.

${ }^{13} \mathrm{C}$ NMR (100 MHz, $\left.\mathrm{CDCl}_{3}\right): \delta=194.6,162.8$ (d, C-F, $\left.{ }^{1} J=246 \mathrm{~Hz}\right), 153.1,149.6,146.3$, 141.6, 140.5, 138.3, 137.8, 137.7 (d, C-F, $\left.{ }^{4} J=3.7 \mathrm{~Hz}\right), 137.2,135.4,133.4\left(\mathrm{~d}, \mathrm{C}-\mathrm{F},{ }^{3} \mathrm{~J}=7.6 \mathrm{~Hz}\right)$, 133.0, 129.2, 128.9 (d, C-F, $\left.{ }^{2} J=21.2 \mathrm{~Hz}\right), 128.8,128.3,128.03,127.97,127.1,125.4,124.4$, $115.4,115.2,107.8,107.0,56.4(2 x)$, and -1.6.

IR (neat): 3044, 3000, 2952, 2899, 2835, 1705, 1247, 1029, 1015, and $771 \mathrm{~cm}^{-1}$. HRMS (ESI) m/z: $[\mathrm{M}+\mathrm{H}]^{+}$Calcd for $\mathrm{C}_{32} \mathrm{H}_{28} \mathrm{FO}_{3} \mathrm{Si}$ 507.1786; Found 507.1736. mp: $208-210^{\circ} \mathrm{C}$. 


\section{4-(11,12-Dimethoxy-9-oxo-8-(trimethylsilyl)-9H-indeno[2,1-c]phenanthren-7- yl)benzonitrile (19b)}

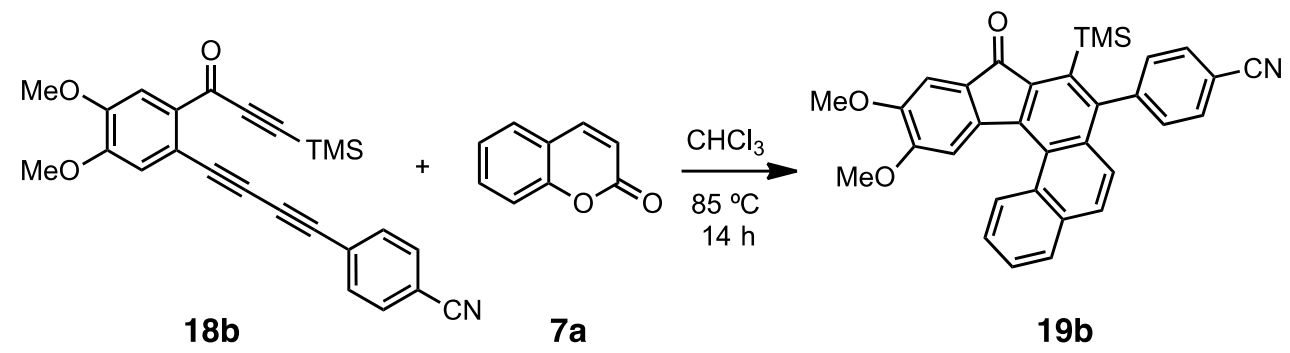

Following general procedure C: Triyne $\mathbf{1 8 b}$ (30 mg, $0.07 \mathrm{mmol})$ and 2H-chromen-2-one (7a, 56 $\mathrm{mg}, 0.22 \mathrm{mmol})$ were dissolved in $\mathrm{CHCl}_{3}(3 \mathrm{~mL})$ to prepare the phenanthrene derivative $\mathbf{1 9 b}$. Purification of the crude material by MPLC (hexanes:EtOAc, 9:1) provided 19b (12 mg, 0.023 mmol, 33\%) as a red crystalline solid.

${ }^{1}$ H NMR (400 MHz, $\left.\mathrm{CDCl}_{3}\right): \delta=8.93(\mathrm{dd}, J=8.3,0.9 \mathrm{~Hz}, 1 \mathrm{H}, H 1), 7.84(\mathrm{dd}, J=8.0,1.5 \mathrm{~Hz}, 1 \mathrm{H}$, H4), $7.79\left(\right.$ nfod, $\left.J_{a p p}=8.3 \mathrm{~Hz}, 2 \mathrm{H}, H_{o}\right), 7.66(\mathrm{~s}, 1 \mathrm{H}, H 13), 7.64(\mathrm{ddd}, J=8.2,7.0,1.0 \mathrm{~Hz}, 1 \mathrm{H}, H 3)$, 7.57 (br d, $J=9.2 \mathrm{~Hz}, 1 \mathrm{H}, H 5), 7.54(\mathrm{ddd}, J=8.4,7.1,1.5 \mathrm{~Hz}, 1 \mathrm{H}, H 2), 7.44\left(\mathrm{nfod}, J_{a p p}=8.4 \mathrm{~Hz}\right.$, 2H, $\left.H_{m}\right), 7.21(\mathrm{~s}, 1 \mathrm{H}, H 10), 7.09$ (d, J = 9.3 Hz, 1H, H6), 3.96 (s, 3H, CH3 O ), 3.84 (s, 3H, CH3O'), and $0.00\left(\mathrm{~s}, 9 \mathrm{H}, \mathrm{Si}\left(\mathrm{CH}_{3}\right)_{3}\right)$.

${ }^{13} \mathrm{C}$ NMR (100 MHz, $\left.\mathrm{CDCl}_{3}\right): \delta=194.4,153.2,149.8,147.0,145.0,142.2,140.3,138.3,136.8$, 134.9, 133.2, 132.7, 132.1, 129.2, 129.1, 128.9, 128.6, 128.08, 128.06, 127.0, 124.8, 124.7, $118.9,112.0,107.8,107.0,56.4(2 \mathrm{x})$, and 1.8.

IR (neat): 3052, 3001, 2952, 2899, 2835, 2228, 1702, 1156, 1113, 1020, 999, 883, and $764 \mathrm{~cm}^{-1}$. HRMS (ESI) m/z: [M + H] $]^{+}$Calcd for $\mathrm{C}_{33} \mathrm{H}_{28} \mathrm{NO}_{3} \mathrm{Si}$ 514.1838; Found 514.1828.

mp: $148-150{ }^{\circ} \mathrm{C}$. 


\section{7-(4-Acetylphenyl)-11,12-dimethoxy-8-(trimethylsilyl)-9H-indeno[2,1-c]phenanthren-9-one} (19c)

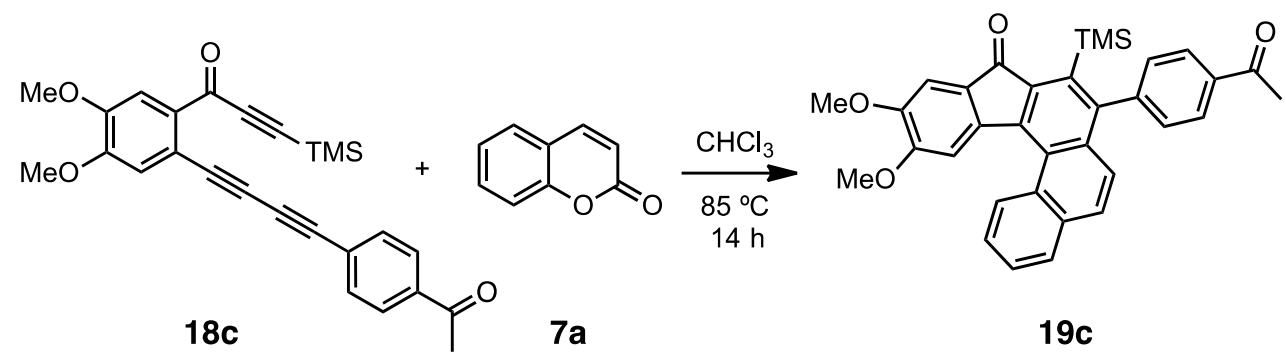

Following general procedure C: Triyne $\mathbf{1 8 c}(50 \mathrm{mg}, 0.116 \mathrm{mmol})$ and $2 \mathrm{H}$-chromen-2-one (7a, $51 \mathrm{mg}, 0.350 \mathrm{mmol})$ were dissolved in $\mathrm{CHCl}_{3}(3 \mathrm{~mL})$ and used to prepare the phenanthrene derivative 19c. Purification of the crude material by MPLC (hexanes:EtOAc, 9:1) provided 19c (16 mg, $0.030 \mathrm{mmol}, 26 \%)$ as a red crystalline solid.

${ }^{1}$ H NMR (400 MHz, $\mathrm{CDCl}_{3}$ ): $\delta=8.92(\mathrm{dddd}, J=8.3,1.2,0.7,0.7 \mathrm{~Hz}, 1 \mathrm{H}, H 1), 8.08$ (nfod, $J_{a p p}=$ $\left.8.5 \mathrm{~Hz}, 2 \mathrm{H}, \mathrm{Ar} H_{o}\right), 7.83$ (dd, $\left.J=8.0,1.5 \mathrm{~Hz}, 1 \mathrm{H}, H 4\right), 7.66$ (s, 1H, H13), 7.63 (ddd, J = 8.1, 7.1, $1.2 \mathrm{~Hz}, 1 \mathrm{H}, H 3), 7.54$ (br d, $J=9.1 \mathrm{~Hz}, 1 \mathrm{H}, H 5), 7.53$ (ddd, $J=8.4,7.0,1.4 \mathrm{~Hz}, 1 \mathrm{H}, H 2$ ), 7.43 (d, $\left.J_{a p p}=8.4 \mathrm{~Hz}, 2 \mathrm{H}, \mathrm{ArH2}\right), 7.21(\mathrm{~s}, 1 \mathrm{H}, \mathrm{H} 13), 7.17(\mathrm{~d}, J=9.3 \mathrm{~Hz}, 1 \mathrm{H}, H 6), 3.96(\mathrm{~s}, 3 \mathrm{H}, \mathrm{CH} 3 \mathrm{O})$, 3.84 (s, 3H, $\mathrm{CH}_{3} \mathrm{O}$ '), 2.71 (s, 3H, 4- $\left.\mathrm{ArCOCH}_{3}\right)$, and 0.01 (s, 9H, $\left.\mathrm{Si}\left(\mathrm{CH}_{3}\right)_{3}\right)$.

${ }^{13} \mathrm{C}$ NMR (100 MHz, $\left.\mathrm{CDCl}_{3}\right): \delta=198.0,194.5,153.2,149.7,147.0,146.1,141.8,140.5,138.3$, $136.8,136.6,135.0,133.3,132.6,132.2$, 132.1, 129.2, 128.92, 128.91, 128.4, 128.2, 128.02, $128.01,127.1,125.2,124.5,107.8,107.0,56.4(2 x), 26.9$ and 1.7.

IR (neat): 3048, 3001, 2952, 2926, 2899, 2871, 2853, 2835, 1702, 1682, 1179, 840, and $735 \mathrm{~cm}^{-1}$. HRMS (ESI) m/z: [M + H] ${ }^{+}$Calcd for $\mathrm{C}_{34} \mathrm{H}_{31} \mathrm{O}_{4} \mathrm{Si}$ 531.1986; Found 531.1934.

mp: $248-250{ }^{\circ} \mathrm{C}$. 


\section{1,12-Dimethoxy-7-(4-methoxyphenyl)-8-(trimethylsilyl)-9H-indeno[2,1-c]phenanthren-9-} one (19d)

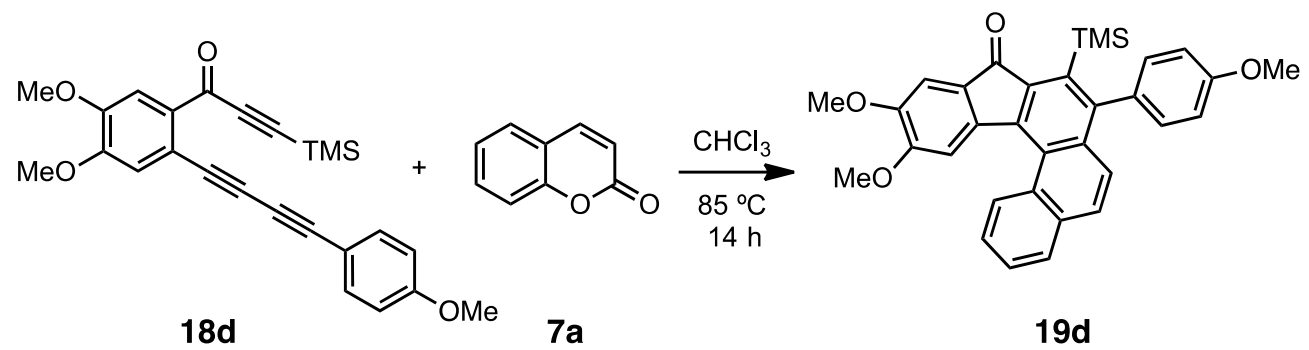

Following general procedure $\mathbf{C}$ : Triyne $19 d(50 \mathrm{mg}, 0.12 \mathrm{mmol})$ and $2 \mathrm{H}$-chromen-2-one (7a, 53 $\mathrm{mg}, 0.36 \mathrm{mmol})$ were dissolved in $\mathrm{CHCl}_{3}(5 \mathrm{~mL})$ and used to prepare the phenanthrene derivative 18d. Purification of the crude material by MPLC (hexanes:EtOAc, 9:1) provided 18d (18 mg, $0.030 \mathrm{mmol}, 25 \%)$ as a red crystalline solid.

${ }^{1} \mathbf{H}$ NMR (400 MHz, $\left.\mathrm{CDCl}_{3}\right): \delta=8.90$ (br d, $\left.J=8.3 \mathrm{~Hz}, 1 \mathrm{H}, H 1\right), 7.82$ (br dd, $J=7.9,1.3 \mathrm{~Hz}, 1 \mathrm{H}$, H4), 7.65 (s, 1H, H13), 7.61 (ddd, $J=8.0,7.0,1.2 \mathrm{~Hz}, 1 \mathrm{H}, H 3), 7.55$ (br d, $J=9.2 \mathrm{~Hz}, 1 \mathrm{H}, H 5$ ), $7.51(\mathrm{ddd}, J=8.3,7.0,1.4 \mathrm{~Hz}, 1 \mathrm{H}, H 2), 7.36(\mathrm{~d}, J=9.3 \mathrm{~Hz}, 1 \mathrm{H}, H 6), 7.204($ nfod, $J=8.7 \mathrm{~Hz}, 2 \mathrm{H}$, $\left.H_{m}\right), 7.200(\mathrm{~s}, 1 \mathrm{H}, H 1), 7.00\left(\mathrm{nfod}, J_{a p p}=8.6 \mathrm{~Hz}, 2 \mathrm{H}, H_{o}\right), 3.95\left(\mathrm{~s}, 3 \mathrm{H}, \mathrm{CH}_{3} \mathrm{O}\right), 3.92\left(\mathrm{~s}, 3 \mathrm{H}, \mathrm{CH}_{3} \mathrm{O}^{\prime}\right)$, 3.83 (s, 3H, $\mathrm{CH}_{3} \mathrm{O}$ ') $)$, and $0.03\left(\mathrm{~s}, 9 \mathrm{H}, \mathrm{Si}\left(\mathrm{CH}_{3}\right)_{3}\right)$.

${ }^{13} \mathrm{C}$ NMR (100 MHz, $\left.\mathrm{CDCl}_{3}\right): \delta=194.8,159.6,153.1,149.5,147.3,141.2,140.7,138.3,137.4$, 135.6, 134.1, 133.4, 132.9, 129.3, 128.9, 128.4, 128.2, 128.0, 127.9, 127.1, 125.8, 124.3, 113.6, $107.7,107.0,56.3(2 x), 55.5$, and 1.7 .

IR (neat): 3040, 3000, 2952, 2899, 2835, 1732, 1702, 1217, 1031, and $738 \mathrm{~cm}^{-1}$.

HRMS (ESI) m/z: [M + Na $]^{+}$Calcd for $\mathrm{C}_{33} \mathrm{H}_{30} \mathrm{NaO}_{4} \mathrm{Si} 541.1811$; Found 541.1784.

mp: $252-254{ }^{\circ} \mathrm{C}$. 


\section{Methyl 4-(11,12-Dimethoxy-9-oxo-8-(trimethylsilyl)-9H-indeno[2,1-c]phenanthren-7- yl)benzoate (19e-syn)}

and

Methyl 4-(9,10-dimethoxy-7-oxo-6-(trimethylsilyl)-7H-indeno[1,2-a]phenanthren-5yl)benzoate (19e-anti)

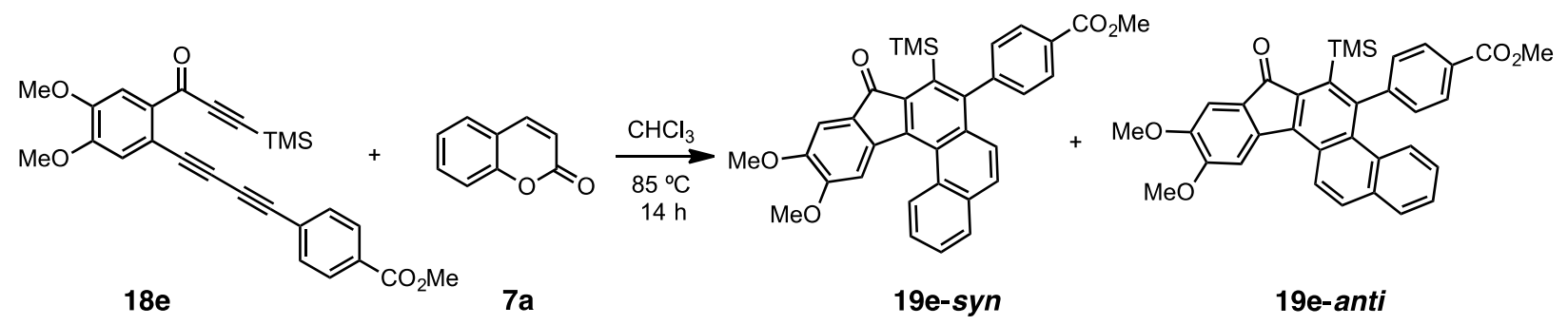

Following general procedure C: Triyne 18e (444 mg, $1.0 \mathrm{mmol})$ and 2H-chromen-2-one $2 \mathrm{H}-$ chromen-2-one (7a, $438 \mathrm{mg}, 3.0 \mathrm{mmol})$ were dissolved in $\mathrm{CHCl}_{3}(30 \mathrm{~mL})$ to prepare the phenanthrene 19e-syn and phenanthrene 19e-anti derivatives. Purification of the crude material by MPLC (hexanes:EtOAc, 9:1) provided, in order of elution, the phenanthrene 19e-syn (135 mg, $0.247 \mathrm{mmol}, 25 \%$ ) as an orange crystalline solid followed by the isomeric phenanthrene 19e-anti (45 $\mathrm{mg}, 0.08 \mathrm{mmol}, 8 \%$ ) as a pale orange crystalline solid.

\section{Data for 19e-syn}

${ }^{1} \mathbf{H}$ NMR $\left(400 \mathrm{MHz}, \mathrm{CDCl}_{3}\right): \delta=8.92(\mathrm{dd}, J=8.0,1.0 \mathrm{~Hz}, 1 \mathrm{H}, H 1), 8.08\left(\mathrm{nfod}, J_{a p p}=8.5 \mathrm{~Hz}, 2 \mathrm{H}\right.$, ArH2'), 7.83 (dd, $J=8.0,1.3 \mathrm{~Hz}, 1 \mathrm{H}, H 4), 7.66$ (s, $1 \mathrm{H}, H 13), 7.63$ (ddd, $J=8.2,7.0,1.2 \mathrm{~Hz}, 1 \mathrm{H}$, $H 2$ or $H 3$ ), 7.54 (br d, $J=9.1 \mathrm{~Hz}, 1 \mathrm{H}, H 5), 7.52$ (ddd, $J=8.3,7.1,1.4 \mathrm{~Hz}, 1 \mathrm{H}, H 2$ or $H 3$ ), 7.40 (nfod, Japp $=8.4 \mathrm{~Hz}, 2 \mathrm{H}, \mathrm{ArH3}$ '), $7.21(\mathrm{~s}, 1 \mathrm{H}, H 10), 7.17(\mathrm{~d}, J=9.1 \mathrm{~Hz}, 1 \mathrm{H}, H 6), 3.99$ (s, 3H, $\left.\mathrm{CH}_{3} \mathrm{O}\right), 3.96\left(\mathrm{~s}, 3 \mathrm{H}, \mathrm{CH}_{3} \mathrm{O}^{\prime}\right), 3.84\left(\mathrm{~s}, 3 \mathrm{H}, 4-\mathrm{ArCOCH}_{3}\right)$, and 0.01 (s, 9H, $\left.\mathrm{Si}\left(\mathrm{CH}_{3}\right)_{3}\right)$.

${ }^{13} \mathrm{C}$ NMR (100 MHz, $\left.\mathrm{CDCl}_{3}\right): \delta=194.5,167.1,153.2,149.7,146.8,146.2,141.8,140.5,138.3$, $136.9,135.0,133.3,132.0,129.7,129.5,129.2$, 128.91, 128.89, 128.4, 128.0, 127.1, 125.2, 124.5, 107.8, 107.0, 56.4 (2x), 52.4, and 1.7 (one aromatic resonance not discernible).

IR (neat): 3043, 2999, 2951, 2901, 2836, 1723, 1705, 1248, 1101, and $809 \mathrm{~cm}^{-1}$. HRMS (ESI) m/z: [M + H] ${ }^{+}$Calcd for $\mathrm{C}_{34} \mathrm{H}_{31} \mathrm{O}_{5} \mathrm{Si}$ 547.1941; Found 547.1928. mp: $272-274{ }^{\circ} \mathrm{C}$. 


\section{Data for 19e-anti}

${ }^{1} \mathbf{H}$ NMR $\left(400 \mathrm{MHz}, \mathrm{CDCl}_{3}\right): \delta=8.24(\mathrm{~d}, J=9.0 \mathrm{~Hz}, 1 \mathrm{H}, H 12), 8.14\left(\right.$ nfod, $J_{a p p}=8.0 \mathrm{~Hz}, 2 \mathrm{H}$, ArH2'), $7.80(\mathrm{~d}, J=9.1 \mathrm{~Hz}, 1 \mathrm{H}, H 13), 7.77(\mathrm{dd}, J=8.0,1.5 \mathrm{~Hz}, 1 \mathrm{H}, H 1), 7.54$ (s, $1 \mathrm{H}, H 11)$, $7.43\left(\right.$ nfod, $_{\text {app }}=8.0 \mathrm{~Hz}, 2 \mathrm{H}, \mathrm{ArH3}$ ') 7.39 (dddd, $\left.J=7.9,6.9 .0 .9,0.9 \mathrm{~Hz}, 1 \mathrm{H}, H 2\right), 7.26$ (s, $1 \mathrm{H}$, H8), 7.05 (d, $J=8.7 \mathrm{~Hz}, 1 \mathrm{H}, H 4), 6.93$ (ddd, $J=8.6,6.9,1.7 \mathrm{~Hz}, 1 \mathrm{H}, H 3), 4.11(\mathrm{~s}, 3 \mathrm{H}, \mathrm{CH} 3 \mathrm{O}$ ), 4.00 (s, 3H, $\mathrm{CH}_{3} \mathrm{O}$ '), 3.97 (s, 3H, CH3O'), and 0.02 (s, 9H, $\left.\mathrm{Si}\left(\mathrm{CH}_{3}\right)_{3}\right)$.

${ }^{13}$ C NMR (100 MHz, $\left.\mathrm{CDCl}_{3}\right): \delta=195.2,167.2,153.9,150.4,149.6,146.4,140.9,139.1,138.4$, 138.0, 134.5, 133.5, 131.9, 131.0, 130.5, 129.8, 129.7, 128.7, 128.2, 127.8, 127.1, 125.1, 121.6, $56.7,56.4,52.4,2.3$, and 0.1 . Three carbon resonances not clearly observed; several weak resonances at ca. 107.5, presumably reflect slow biaryl-rotation and/or slow helical conformational interconversion on the NMR time scale.

IR (neat): 3053, 2977, 2915, 2854, 1715, 1596, 1473, 1393, 1295, 1253, 1228, 1105, 987, 878, 810,784 and $744 \mathrm{~cm}^{-1}$.

HRMS (ESI) m/z: [M + H] ${ }^{+}$Calcd for $\mathrm{C}_{34} \mathrm{H}_{31} \mathrm{O}_{5} \mathrm{Si}$ 547.1941; Found 547.1914. mp: $260-262{ }^{\circ} \mathrm{C}$. 


\section{1,12-Dimethoxy-7-methyl-8-(trimethylsilyl)-9H-indeno[2,1-c]chrysen-9-one (21a)}

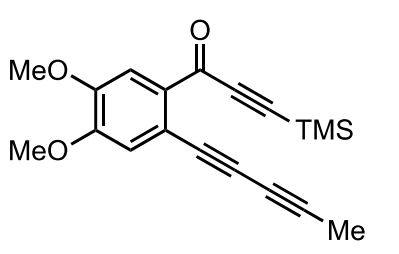

13

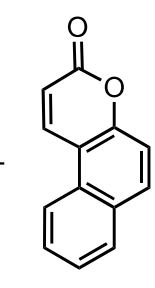

$20 a$

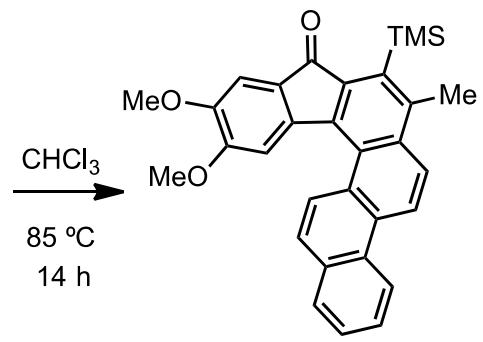

$21 a$

Following general procedure C: Triyne 13 (50 mg, $0.154 \mathrm{mmol})$ and 3H-benzo[f]chromen-3one ${ }^{13}$ (20a, $\left.90 \mathrm{mg}, 0.462 \mathrm{mmol}\right)$ were dissolved in $(5 \mathrm{~mL})$ of $\mathrm{CHCl}_{3}$ and used to prepare chrysenone derivative 21a. Purification of the crude material by MPLC (hexanes:EtOAc, 9:1) provided 21a (30 mg, $0.063 \mathrm{mmol}, 41 \%$ ) as a brown crystalline solid.

${ }^{1} \mathbf{H}$ NMR $\left(400 \mathrm{MHz}_{\mathrm{CDCl}}\right)$ ): $\delta=8.79(\mathrm{~d}, J=9.0 \mathrm{~Hz}, 1 \mathrm{H}, H 14), 8.75(\mathrm{~d}, J=8.4,1 \mathrm{H}, H 4), 8.69$ (d, $J=9.5 \mathrm{~Hz}, 1 \mathrm{H}, H 5), 8.14(\mathrm{~d}, J=9.4 \mathrm{~Hz}, 1 \mathrm{H}, H 6), 7.99$ (d, $J=8.1 \mathrm{~Hz}, 1 \mathrm{H}, 1 H), 7.83(\mathrm{~d}, J=$ $8.8 \mathrm{~Hz}, 1 \mathrm{H}, H 15), 7.75(\mathrm{ddd}, J=8.5,7.0,1.5 \mathrm{~Hz}, 1 \mathrm{H}, H 3), 7.68(\mathrm{ddd}, J=8.1,7.0,1.3 \mathrm{~Hz}, 1 \mathrm{H}$, H2), 7.50 (s, 1H, H13), 7.17 (s, $1 \mathrm{H}, \mathrm{H} 10$ ), 3.93 (s, 3H, $\mathrm{CH}_{3} \mathrm{O}$ ), 3.80 (s, 3H, $\mathrm{CH}_{3} \mathrm{O}$ '), 2.85 (s, 3H, $\left.\mathrm{ArCH}_{3}\right)$, and $0.51\left(\mathrm{~s}, 9 \mathrm{H}, \mathrm{Si}\left(\mathrm{CH}_{3}\right)_{3}\right)$.

${ }^{13} \mathrm{C}$ NMR (100 MHz, $\left.\mathrm{CDCl}_{3}\right): \delta=195.0,153.1,149.1,142.5,141.0,140.4,138.2,136.6,135.3$, $132.8,130.2,130.0,128.7,128.0,127.9,127.6,127.1,127.1,126.4,124.4,123.7,123.7,123.4$, 108.2, 107.0, 56.4, 56.3, 21.6, and 2.6.

IR (neat): 3388, 3077, 3052, 3001, 2947, 2899, 2834, 1698, 1245, 994, 703, 677, and $631 \mathrm{~cm}^{-1}$. HRMS (ESI) m/z: [M + H] $]^{+}$Calcd for $\mathrm{C}_{31} \mathrm{H}_{29} \mathrm{O}_{3} \mathrm{Si}$ 477.1880; Found 477.1889.

mp: $240-242{ }^{\circ} \mathrm{C}$. 


\section{2-Bromo-11,12-dimethoxy-7-methyl-8-(trimethylsilyl)-9H-indeno[2,1-c]chrysen-9-one (21b)}

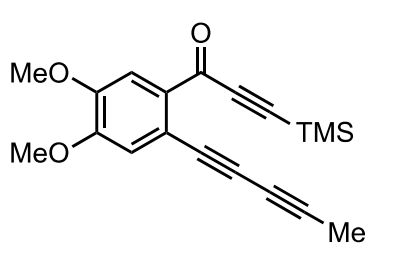

13

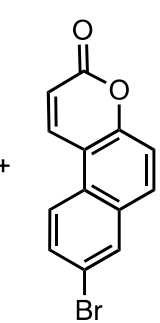

$20 b$

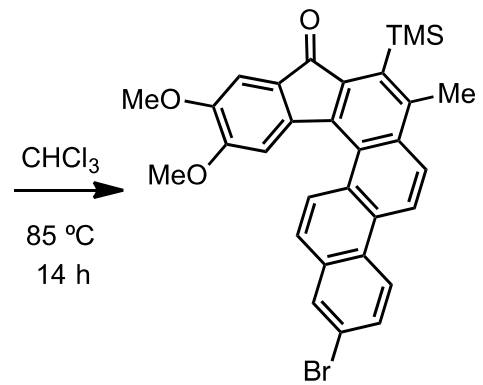

21b

Following general procedure C: Triyne 13 (30 mg, $0.09 \mathrm{mmol}$ ) and 8-bromo-3Hbenzo[f]chromen-3-one ${ }^{14}(\mathbf{2 0 b}, 76 \mathrm{mg}, 0.27 \mathrm{mmol})$ were dissolved in $(3 \mathrm{~mL})$ of $\mathrm{CHCl}_{3}$ and used to prepare the chrysenone derivative $\mathbf{2 1 b}$. Purification of the crude material by MPLC (hexanes: EtOAc, 9:1) provided $\mathbf{2 1 b}$ (15 mg, $0.027 \mathrm{mmol}, 30 \%$ ) as a red crystalline solid. (see page S65 for the single crystal x-ray diffraction). The sample was grown by vapor diffusion of a solution of DCM and pentane.

${ }^{1} \mathbf{H}$ NMR $\left(400 \mathrm{MHz}, \mathrm{CDCl}_{3}\right): \delta=8.82(\mathrm{~d}, J=9.1 \mathrm{~Hz}, 1 \mathrm{H}, H 14), 8.60$ (two overlapped ds, $J=9$ $\mathrm{Hz}, 2 \mathrm{H}, H 4$ and $H 5), 8.16(\mathrm{~d}, J=9.4 \mathrm{~Hz}, 1 \mathrm{H}, H 15), 8.14(\mathrm{~d}, J=2.1 \mathrm{~Hz}, 1 \mathrm{H}, H 1), 7.82(\mathrm{dd}, J=$ 9.0, 2.2 Hz, 1H, H3), 7.76 (br d, J= 8.9 Hz, 1H, H6), 7.44 (s, $1 \mathrm{H}, H 13$ ), 7.17 (s, 1H, H1O), 3.93 (s, 3H, $\left.\mathrm{CH}_{3} \mathrm{O}\right), 3.80$ (s, 3H, $\mathrm{CH}_{3} \mathrm{O}$ ') 2.85 (s, 3H, $\left.\mathrm{ArCH}_{3}\right)$, and 0.50 (s, 9H, $\left.\mathrm{Si}\left(\mathrm{CH}_{3}\right)_{3}\right)$.

${ }^{13} \mathrm{C}$ NMR (100 MHz, $\left.\mathrm{CDCl}_{3}\right): \delta$ 194.9, 153.1, 149.2, 142.6, 140.8, 140.3, 138.4, 137.0, 135.2, $134.1,130.8,130.3,129.8,128.8,128.7,128.0,127.8,126.4,125.3,124.3,123.3,123.2,121.2$, 108.1, 107.1, 56.4, 56.4, 21.6, and 2.6.

IR (neat): 3075, 3002, 2950, 2936, 2901, 2852, 2836, 1702, 1245, 1059, 1018, and $702 \mathrm{~cm}^{-1}$. HRMS (ESI) m/z: $[\mathrm{M}+\mathrm{H}]^{+}$Calcd for $\mathrm{C}_{31} \mathrm{H}_{28} \mathrm{O}_{3}{ }^{79} \mathrm{BrSi}$ 555.0986; Found 555.0974. mp: $118-120^{\circ} \mathrm{C}$. 


\section{1,12-Dimethoxy-7-methyl-2-phenyl-8-(trimethylsilyl)-9H-indeno[2,1-c]chrysen-9-one (21c)}

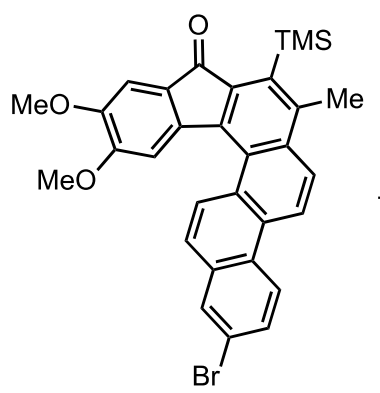

20b

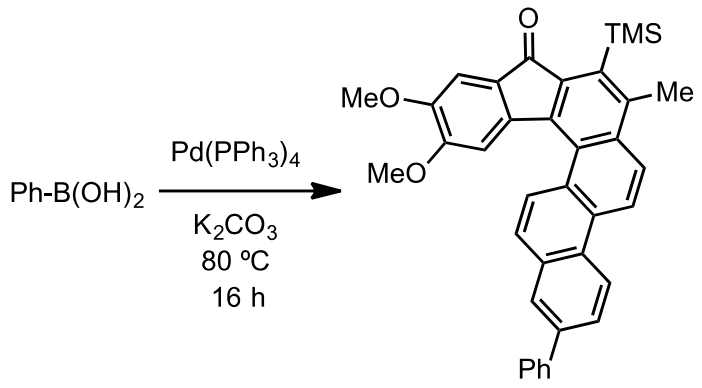

21c

To a solution of the bromochrysenone $20 \mathrm{~b}$ (25 mg, $0.045 \mathrm{mmol})$ in anhydrous methanol (1.0 $\mathrm{mL})$, anhydrous toluene $(1.0 \mathrm{~mL})$ and distilled water $(1.0 \mathrm{~mL})$ were added phenyl boronic acid $(7 \mathrm{mg}$, $0.054 \mathrm{mmol}), \mathrm{K}_{2} \mathrm{CO}_{3}(13 \mathrm{mg}, 0.09 \mathrm{mmol})$ and $\mathrm{Pd}\left(\mathrm{PPh}_{3}\right)_{4}(5 \mathrm{mg}, 0.0045 \mathrm{mmol})$. This mixture was stirred at $80{ }^{\circ} \mathrm{C}$ for $16 \mathrm{~h}$. The reaction mixture was diluted with water $(10 \mathrm{~mL})$ and extracted with ethyl acetate $(10 \mathrm{~mL})$. Evaporation of the solvent and purification of the crude reaction mixture by flash chromatography (9:1 hexanes:EtOAc) gave the chrysen-9-one derivative 21c (23 mg, 0.041 mmol, 91\%) as a brown crystalline solid.

${ }^{1} \mathbf{H}$ NMR (400 MHz, $\left.\mathrm{CDCl}_{3}\right): \delta=8.81(\mathrm{~d}, J=9.0 \mathrm{~Hz}, 1 \mathrm{H}, H 5), 8.80(\mathrm{~d}, J=8.8 \mathrm{~Hz}, 1 \mathrm{H}, H 4)$, $8.69(\mathrm{~d}, J=9.4 \mathrm{~Hz}, 1 \mathrm{H}, H 14), 8.19(\mathrm{~d}, J=2.0 \mathrm{~Hz}, 1 \mathrm{H}, H 1), 8.16(\mathrm{~d}, J=9.3 \mathrm{~Hz}, 1 \mathrm{H}, H 15), 7.99$ (dd, $J=8.7,2.0 \mathrm{~Hz}, 1 \mathrm{H}, H 3), 7.88$ (d, $J=9.0 \mathrm{~Hz}, 1 \mathrm{H}, H 6), 7.81-7.78\left(\mathrm{nfod}, 2 \mathrm{H}, \mathrm{Ph} H_{o}\right), 7.53$ (nfodd, 2H, PhHm), 7.51 (s, 1H, H13), 7.42 (tt, $J=7.4,1.3 \mathrm{~Hz}, 1 \mathrm{H}, \mathrm{Ph} H_{p}$ ), 7.17 (s, 1H, H10), $\left.3.93\left(\mathrm{~s}, 3 \mathrm{H}, \mathrm{CH}_{3} \mathrm{O}\right), 3.81\left(\mathrm{~s}, 3 \mathrm{H}, \mathrm{CH}_{3} \mathrm{O}\right)^{\prime}\right), 2.86\left(\mathrm{~s}, 3 \mathrm{H}, \mathrm{ArCH}_{3}\right)$, and $0.51\left(\mathrm{~s}, 9 \mathrm{H}, \mathrm{Si}\left(\mathrm{CH}_{3}\right)_{3}\right)$

${ }^{13} \mathrm{C}$ NMR (100 MHz, $\left.\mathrm{CDCl}_{3}\right): \delta=195.0,153.1,149.1,142.5,141.0,140.7,140.3,139.8,138.2$, 136.7, 135.3, 133.2, 129.9, 129.3, 129.1, 128.03, 127.97, 127.94, 127.85, 127.5, 126.6, 126.5, $126.4,124.6,124.1,123.9,123.7,108.2,107.0,56.4,56.3,21.6$, and 2.6.

IR (neat): 3056, 3001, 2956, 2900, 2835, 1701, 1586, 1492, 1462, 1314, 1277, 1244, 1214, 1093, 842 , and $761 \mathrm{~cm}^{-1}$.

HRMS (ESI) m/z: [M + H] ${ }^{+}$Calcd for $\mathrm{C}_{37} \mathrm{H}_{33} \mathrm{O}_{3} \mathrm{Si}$ 553.2193; Found 553.2183. mp: $218-220{ }^{\circ} \mathrm{C}$. 


\section{Phenanthrene (9) and biphenylene (23)}

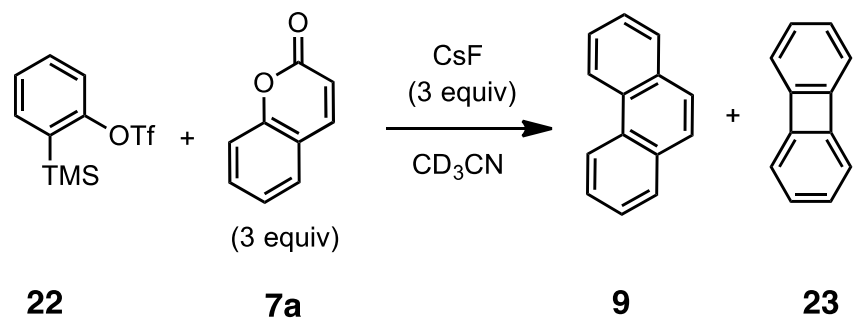

2-(Trimethylsilyl)phenyl trifluoromethanesulfonate ${ }^{15} 22$ (30 mg, $\left.0.10 \mathrm{mmol}\right)$ and coumarin (44 $\mathrm{mg}, 0.3 \mathrm{mmol})$ and $\mathrm{CsF}(45 \mathrm{mg}, 0.3 \mathrm{mmol})$ were dissolved in $\mathrm{CH}_{3} \mathrm{CN}(3.0 \mathrm{~mL})$ or $\mathrm{CD}_{3} \mathrm{CN}(1.5$ $\mathrm{mL}$ ) and stirred at room temperature for $14 \mathrm{~h}$. Purification of the crude material by MPLC (hexanes:EtOAc, 9:1) provided a coeluting mixture of phenanthrene (9) and biphenylene (23) (1:0.14) (10 mg, $0.056 \mathrm{~mol}, 19 \%)$ as a yellow liquid. The presence of each was verified by analysis of proton NMR and GCMS data for the reaction solution in $\mathrm{CD}_{3} \mathrm{CN}$.

From the proton NMR spectrum of that reaction mixture, provided on page S125, it is clear that 9 and $\mathbf{2 3}$ are, by far, the predominant products formed in this reaction; their ratio, deduced from analysis of this spectrum, was 4.8:1. More specifically, the resonances of all $\mathrm{Me}_{3} \mathrm{Si}$-containing byproducts are essentially identical in their intensity (integration) to those of the moieties to which $\mathbf{2 2}$ gives rise in $\mathbf{9}$ (from 1 molecule of $\mathbf{2 2}$ ) and $\mathbf{2 3}$ (from two molecules of 22).

\section{Phenanthrene (9)}

${ }^{1} \mathbf{H}$ NMR (400 MHz, $\left.\mathrm{CDCl}_{3}\right): \delta=8.70(\mathrm{dd}, J=8.3,1.4 \mathrm{~Hz}, 2 \mathrm{H}, H 4), 7.89(\mathrm{dd}, J=7.9,1.4 \mathrm{~Hz}$, $2 \mathrm{H}, H 1), 7.74(\mathrm{~s}, 2 \mathrm{H}, H 9), 7.66(\mathrm{ddd}, J=8.5,7.0,1.0 \mathrm{~Hz}, 2 \mathrm{H}, H 3)$, and $7.60(\mathrm{ddd}, J=8.0,7.0$, $1.3,2 \mathrm{H}, \mathrm{H} 2)$.

\section{Biphenylene (23)}

${ }^{1} \mathbf{H}$ NMR (400 MHz, $\mathrm{CDCl}_{3}$ ): $\delta=6.73$ (nfom, $1 \mathrm{H}, H_{\text {aa' }}$ of an aa'bb' spin system), and 6.63 (nfom, $\mathrm{H}, H_{\mathrm{bb}}$ ' of an aa'bb' spin system).

LRMS (from GCMS analysis; electron ionization at $70 \mathrm{eV} ; \mathrm{m} / z): 23 \mathrm{t}_{\mathrm{R}}=6.9 \mathrm{~min}(152,100 \% \mathrm{rel}$ int); $9 \mathrm{t}_{\mathrm{R}}=8.8 \mathrm{~min}(178,100 \%$ rel int. $)$. 


\section{Computational methods}

The DFT calculations were performed using Gaussian 09 Revision E.01. The geometry of each structure (including the transition states) was optimized at the B3LYP/6-311G(d,p) level of theory with the SMD (chloroform) solvation model. The nature of the optimized structure was verified by a frequency calculation ( $298 \mathrm{~K}$ or $358 \mathrm{~K}$ at the same level of theory). Natural bond orbital (NBO) analysis was performed using pop=NBO at the B3LYP/6-311G(d,p) level of theory. Reported below for each structure are the zero-point correction and the thermal correction to the free energy, the sum of the electronic and thermal free energies, and the Cartesian coordinates of the geometry-optimized structures.
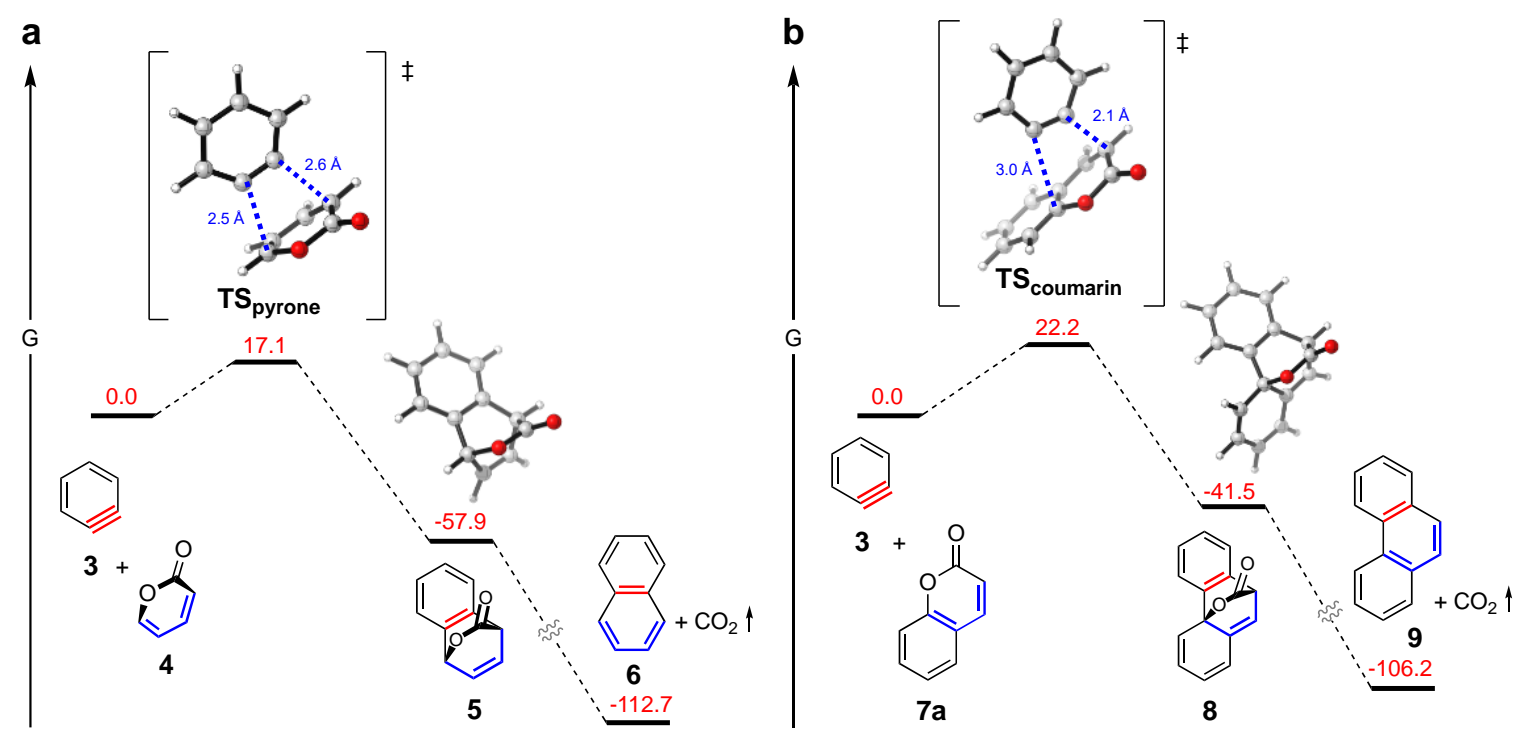

Supplementary Figure 1 (cf. Figure 2 in the manuscript). DFT calculations [B3LYP/6-311G+(d,p), SMD chloroform] of the reaction of $o$-benzyne (3) with a) pyrone (4) and b) coumarin (7a). 


\section{Benzyne (3)}

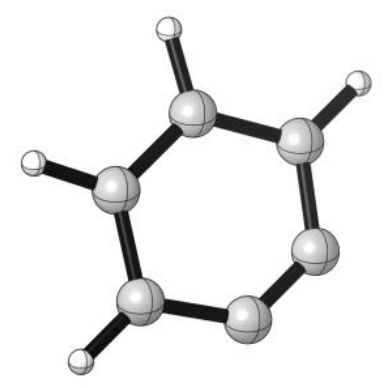

Zero-point correction $=074728$ (Hartree/Particle)

Thermal correction to Gibbs Free Energy $=0.047347$

Sum of electronic and thermal Free Energies $=-230.934271$

\begin{tabular}{|c|c|c|c|c|}
\hline \multirow{2}{*}{$\begin{array}{l}\text { Center } \\
\text { Number }\end{array}$} & \multirow{2}{*}{$\begin{array}{l}\text { Atomic } \\
\text { Number }\end{array}$} & \multirow{2}{*}{$\begin{array}{l}\text { Atomic } \\
\text { Type }\end{array}$} & \multicolumn{2}{|c|}{ Coordinates (Angstr } \\
\hline & & & $\mathrm{X}$ & $\mathrm{Y} \quad \mathrm{Z}$ \\
\hline 1 & 0 & 0.702757 & 1.055811 & -0.000010 \\
\hline 2 & 0 & -0.702772 & 1.055802 & 0.000033 \\
\hline 3 & 0 & -1.462329 & -0.133799 & -0.000052 \\
\hline 4 & 0 & -0.622466 & -1.233661 & 0.000016 \\
\hline 5 & 0 & 0.622486 & -1.233662 & 0.000035 \\
\hline 6 & 0 & 1.462328 & -0.133780 & -0.000028 \\
\hline 7 & 0 & 1.228306 & 2.005742 & -0.000004 \\
\hline 8 & 0 & -1.228333 & 2.005727 & 0.000085 \\
\hline 9 & 0 & -2.544497 & -0.135882 & -0.000023 \\
\hline 10 & 0 & 2.544496 & -0.135850 & -0.000022 \\
\hline
\end{tabular}




\section{Pyrone (4)}

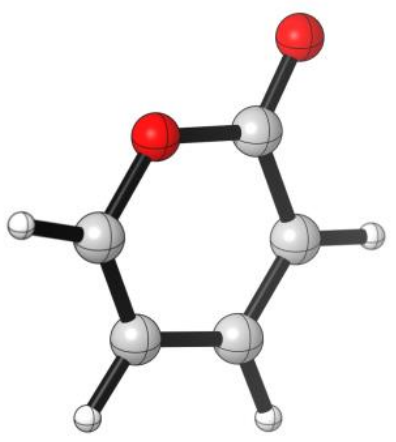

Zero-point correction $=\quad 0.079908($ Hartree/Particle $)$

Thermal correction to Gibbs Free Energy $=0.050924$

Sum of electronic and thermal Free Energies $=\quad-343.427167$

\begin{tabular}{|c|c|c|c|c|c|}
\hline \multirow{3}{*}{$\begin{array}{l}\text { Center } \\
\text { Number } \\
1\end{array}$} & \multirow{2}{*}{\multicolumn{2}{|c|}{$\begin{array}{l}\text { Atomic } \\
\text { Number }\end{array}$}} & \multirow{3}{*}{$\begin{array}{l}\begin{array}{l}\text { Atomic } \\
\text { Type }\end{array} \\
-1.787414\end{array}$} & \multicolumn{2}{|c|}{ Coordinates (Angstroms) } \\
\hline & & & & X $\quad Y$ & $Y \quad Z$ \\
\hline & 6 & 0 & & -0.077408 & 0.000007 \\
\hline 2 & 6 & 0 & -1.106185 & 1.203587 & 0.000016 \\
\hline 3 & 6 & 0 & 0.243830 & 1.268374 & -0.000026 \\
\hline 4 & 6 & 0 & 1.057224 & 0.042929 & -0.000053 \\
\hline 5 & 8 & 0 & 0.336325 & -1.141863 & 0.000027 \\
\hline 6 & 6 & 0 & -1.027656 & -1.189440 & -0.000022 \\
\hline 7 & 8 & 0 & 2.281133 & -0.044427 & 0.000022 \\
\hline 8 & 1 & 0 & -2.866542 & -0.137614 & 0.000015 \\
\hline 9 & 1 & 0 & -1.685762 & 2.117907 & 0.000039 \\
\hline 10 & 1 & 0 & 0.742973 & 2.223778 & 0.000036 \\
\hline 11 & 1 & 0 & -1.409127 & -2.202007 & -0.000015 \\
\hline
\end{tabular}




\section{Pyrone addition to benzyne (TS pyrone)}

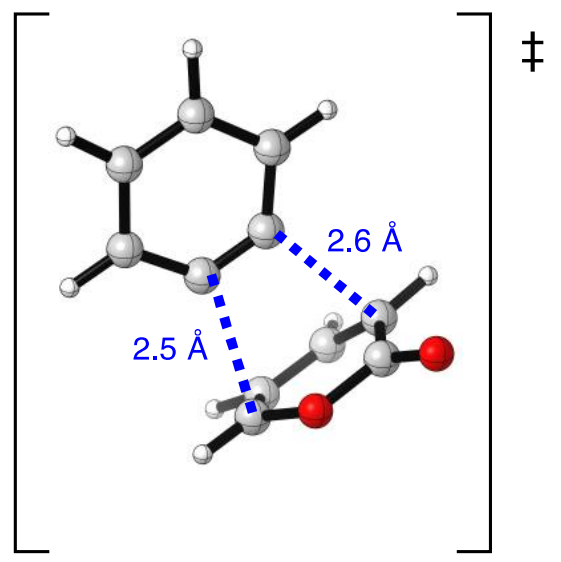

Zero-point correction $=\quad 0.155889($ Hartree/Particle $)$

Thermal correction to Gibbs Free Energy= 0.118317

Sum of electronic and thermal Free Energies $=-574.334174$

\begin{tabular}{cccccc} 
Center & \multicolumn{2}{c}{ Atomic } & \multicolumn{2}{c}{ Atomic } & \multicolumn{3}{c}{ Coordinates (Angstrom } \\
Number & Number & Type & X & $Y$ & $Z$ \\
- & 6 & 0 & 1.901746 & -1.422048 & 0.983973 \\
1 & 6 & 0 & 2.027664 & -1.400054 & -0.426813 \\
3 & 6 & 0 & 1.512124 & -0.228346 & 1.623468 \\
4 & 6 & 0 & 1.097633 & 0.842843 & 0.842104 \\
5 & 6 & 0 & 1.602705 & 0.958233 & -0.533727 \\
6 & 8 & 0 & 2.007693 & -0.218220 & -1.120612 \\
7 & 8 & 0 & 1.683687 & 1.974621 & -1.178884 \\
8 & 1 & 0 & 1.384627 & -0.203372 & 2.699796 \\
9 & 1 & 0 & 0.845238 & 1.789095 & 1.302645 \\
10 & 6 & 0 & -1.779024 & 1.108173 & 0.704279 \\
11 & 6 & 0 & -0.799834 & 0.211145 & 0.303131 \\
12 & 6 & 0 & -0.955594 & -0.896260 & -0.334662 \\
13 & 6 & 0 & -2.217853 & -1.311720 & -0.759515 \\
14 & 6 & 0 & -3.284603 & -0.474711 & -0.394229 \\
15 & 6 & 0 & -3.069287 & 0.711887 & 0.321764 \\
16 & 1 & 0 & -1.600105 & 2.035887 & 1.235348 \\
17 & 1 & 0 & -2.399875 & -2.223863 & -1.319050 \\
18 & 1 & 0 & -4.298285 & -0.753468 & -0.666959 \\
19 & 1 & 0 & -3.912428 & 1.343866 & 0.581191 \\
20 & 1 & 0 & 2.095516 & -2.313030 & 1.543887 \\
21 & 1 & 0 & 2.140210 & -2.321168 & -0.959526 \\
& & & & &
\end{tabular}




\section{Pyrone cycloaddition adduct (5)}

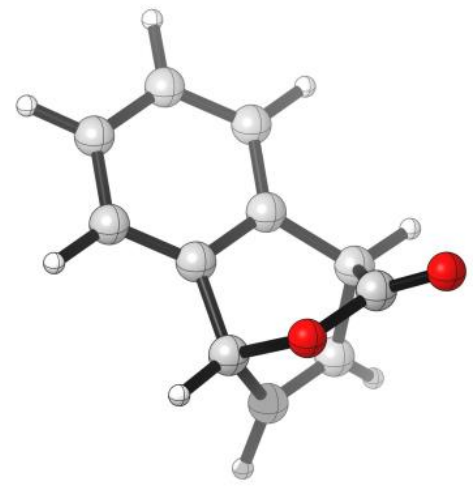

Zero-point correction $=\quad 0.162214$ (Hartree/Particle)

Thermal correction to Gibbs Free Energy $=0.128372$

Sum of electronic and thermal Free Energies $=\quad-574.453766$

\begin{tabular}{|c|c|c|c|c|}
\hline \multirow{2}{*}{$\begin{array}{l}\text { Center } \\
\text { Number }\end{array}$} & Atomic & \multirow{2}{*}{$\begin{array}{r}\text { Atomic } \\
\text { Type }\end{array}$} & \multicolumn{2}{|c|}{ Coordinates (Angstrol } \\
\hline & & & $\begin{array}{ll}X & Y\end{array}$ & $Y \quad Z$ \\
\hline 1 & 6 & 0.640551 & 0.711327 & -0.209164 \\
\hline 2 & 6 & .478545 & -0.583808 & 0.331163 \\
\hline 3 & 6 & -0.980610 & -0.926961 & 0.727462 \\
\hline 4 & 6 & -1.346715 & 0.231010 & 1.685912 \\
\hline 5 & 6 & -1.208 & 1.471810 & 1.153886 \\
\hline 6 & 6 & -0.6 & 1.480581 & -0.309042 \\
\hline 7 & 6 & 13 & 1.156503 & -0.602069 \\
\hline 8 & 6 & 3.009 & 0.317784 & -0.447870 \\
\hline 9 & 6 & 2.854198 & -0.959721 & 0.096317 \\
\hline 10 & 6 & 1.5 & -1.410101 & 0.489526 \\
\hline 11 & 6 & -1.777943 & -0.687434 & -0.592912 \\
\hline 12 & 8 & -2.521038 & -1.458214 & -1.195332 \\
\hline 13 & 8 & -1.622127 & 0.633695 & -1.063376 \\
\hline 14 & 1 & -1.140452 & -1.931162 & 1.115075 \\
\hline 15 & 1 & -1.690152 & 0.070662 & 2.698707 \\
\hline 16 & 1 & -1.428 & 2.373302 & 1.706078 \\
\hline 17 & 1 & -0.600799 & 2.479438 & -0.735768 \\
\hline 18 & 1 & 2.028076 & 2.142392 & -1.025029 \\
\hline 19 & 1 & 3.988803 & 0.657358 & -0.752103 \\
\hline 20 & 1 & 3.714188 & -1.603397 & 0.212848 \\
\hline 21 & 1 & 1.478570 & -2.398375 & 0.910590 \\
\hline
\end{tabular}


Naphthalene (6)

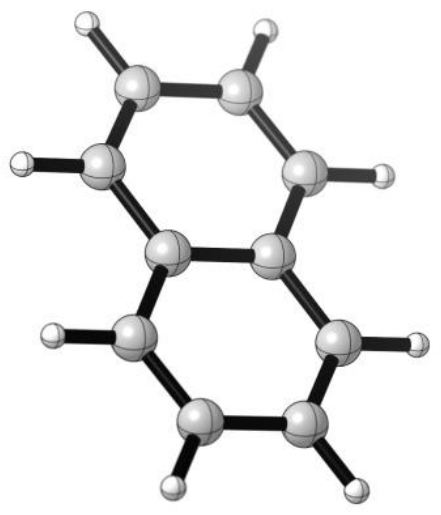

Zero-point correction $=\quad 0.146853($ Hartree/Particle $)$

Thermal correction to Gibbs Free Energy $=0.115632$

Sum of electronic and thermal Free Energies $=-385.886785$

\begin{tabular}{|c|c|c|c|c|c|}
\hline \multirow{3}{*}{$\begin{array}{l}\text { Center } \\
\text { Number } \\
-1\end{array}$} & \multirow{2}{*}{\multicolumn{2}{|c|}{$\begin{array}{l}\text { Atomic } \\
\text { Number }\end{array}$}} & \multirow{3}{*}{$\begin{array}{l}\text { Atomic } \\
\text { Type } \\
0.000066\end{array}$} & \multicolumn{2}{|c|}{ Coordinates (Angstron } \\
\hline & & & & $\begin{array}{ll}X & Y\end{array}$ & $\mathrm{Z}$ \\
\hline & 6 & 0 & & 0.714468 & 0.000017 \\
\hline 2 & 6 & 0 & -0.000032 & -0.714451 & -0.000023 \\
\hline 3 & 6 & 0 & 1.238454 & -1.403703 & 0.000004 \\
\hline 4 & 6 & 0 & 2.438162 & 5543 & 0.000011 \\
\hline 5 & 6 & 0 & 2.438 & 0.695510 & 0.000002 \\
\hline 6 & 6 & & 1.238 & 1.403623 & -0.000008 \\
\hline 7 & 6 & & 0 & 676 & -0.000002 \\
\hline 8 & 6 & 0 & 3217 & 0.695516 & -0.000009 \\
\hline 9 & 6 & 0 & -2.4 & -0.695492 & -0.000008 \\
\hline 10 & 6 & 0 & -1.238 & -1.403605 & 0.000002 \\
\hline 11 & 1 & 0 & 1.2454 & -2.483822 & -0.000007 \\
\hline 12 & 1 & 0 & 3.3775 & -1.229582 & 0.000004 \\
\hline 13 & 1 & & 3.37 & 1.229449 & -0.000013 \\
\hline 14 & 1 & 0 & 1.2455 & 2.483855 & -0.000020 \\
\hline 15 & 1 & 0 & -1.245397 & 2.483840 & 0.000001 \\
\hline 16 & 1 & 0 & -3.377473 & 1.229581 & 0.000087 \\
\hline 17 & 1 & 0 & -3.377586 & -1.229476 & 0.000015 \\
\hline 18 & 1 & 0 & -1.245484 & -2.483837 & 0.000014 \\
\hline
\end{tabular}




\section{Carbon Dioxide $\left(\mathrm{CO}_{2}\right)$}

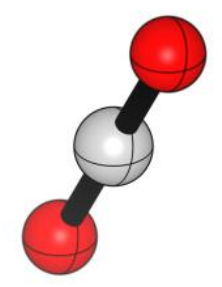

Zero-point correction $=\quad 0.011598($ Hartree/Particle $)$

Thermal correction to Gibbs Free Energy $=\quad-0.009099$

Sum of electronic and thermal Free Energies $=\quad-188.654289$

\begin{tabular}{cccccc} 
Center & \multicolumn{2}{c}{ Atomic } & \multicolumn{2}{c}{ Atomic } & \multicolumn{3}{c}{ Coordinates (Angstrom } \\
Number & Number & Type & X & Y & Z \\
\hline------------- \\
1 & 6 & 0 & 0.000000 & 0.000000 & 0.000000 \\
2 & 8 & 0 & 0.000000 & 0.000000 & 1.258400 \\
3 & 8 & 0 & 0.000000 & 0.000000 & -1.258400
\end{tabular}




\section{Coumarin (7a)}

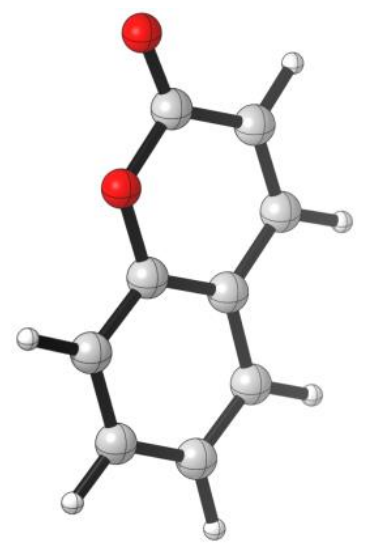

Zero-point correction=

0.126830 (Hartree/Particle)

Thermal correction to Gibbs Free Energy= 0.094175

Sum of electronic and thermal Free Energies= $-497.078795$

\begin{tabular}{ccccccc} 
Center & \multicolumn{2}{c}{ Atomic } & \multicolumn{2}{c}{ Atomic } & \multicolumn{3}{c}{ Coordinates (Angstroms) } \\
Number & Number & Type & X & $Y$ & $Z$ \\
---------------- & \\
1 & 6 & 0 & 1.305987 & -1.473707 & -0.000000 \\
2 & 6 & 0 & 2.618169 & -1.007885 & 0.000013 \\
3 & 6 & 0 & 2.857965 & 0.364654 & -0.000018 \\
4 & 6 & 0 & 1.799690 & 1.271593 & 0.000004 \\
5 & 6 & 0 & 0.487821 & 0.804838 & 0.000036 \\
6 & 6 & 0 & 0.247096 & -0.571342 & -0.000050 \\
7 & 6 & 0 & -0.680111 & 1.686492 & 0.000017 \\
8 & 6 & 0 & -1.928762 & 1.182762 & -0.000037 \\
9 & 6 & 0 & -2.164790 & -0.265230 & 0.000002 \\
10 & 8 & 0 & -1.042805 & -1.087873 & 0.000017 \\
11 & 8 & 0 & -3.254828 & -0.835825 & 0.000002 \\
12 & 1 & 0 & 1.111488 & -2.537252 & 0.000000 \\
13 & 1 & 0 & 3.443683 & -1.706724 & 0.000043 \\
14 & 1 & 0 & 3.876360 & 0.730293 & -0.000044 \\
15 & 1 & 0 & 2.007073 & 2.333990 & -0.000005 \\
16 & 1 & 0 & -0.543230 & 2.760841 & 0.000022 \\
17 & 1 & 0 & -2.772692 & 1.855395 & 0.000025 \\
--------------------------------------------------------
\end{tabular}




\section{Coumarin addition to benzyne (TScoumarin)}

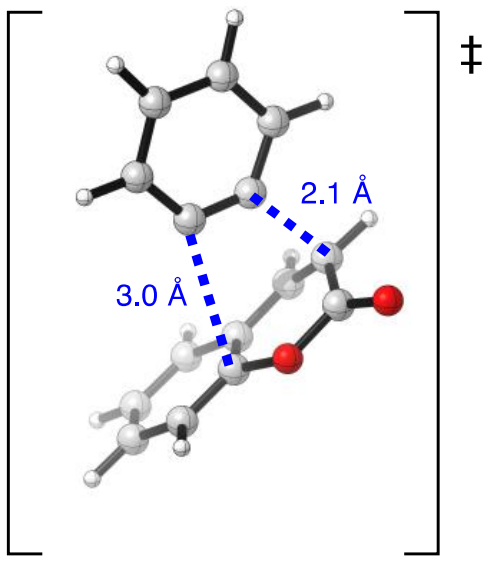

Zero-point correction $=$

0.202639 (Hartree/Particle)

Thermal correction to Gibbs Free Energy=

0.161770

Sum of electronic and thermal Free Energies $=\quad-727.977730$

\begin{tabular}{|c|c|c|c|c|c|}
\hline \multirow{2}{*}{$\begin{array}{l}\text { Center } \\
\text { Number }\end{array}$} & \multirow{2}{*}{\multicolumn{2}{|c|}{$\begin{array}{l}\text { Atomic } \\
\text { Number }\end{array}$}} & \multirow{2}{*}{$\begin{array}{l}\text { Atomic } \\
\text { Type }\end{array}$} & \multicolumn{2}{|c|}{ Coordinates (Angstr } \\
\hline & & & & $\begin{array}{ll}X & Y\end{array}$ & Z \\
\hline 1 & 6 & 0 & 2.681184 & 5.022957 & 11.930832 \\
\hline 2 & 6 & 0 & 3.248136 & 6.215138 & 11.506757 \\
\hline 3 & 6 & 0 & 4.186058 & 6.238488 & 10.458959 \\
\hline 4 & 6 & 0 & 4.558083 & 5.061525 & 9.838586 \\
\hline 5 & 6 & & 4.002762 & 3.827528 & 10.24701 \\
\hline 6 & 6 & & 3.045537 & 3.842539 & 11.285368 \\
\hline 7 & 6 & 0 & 4.361733 & 2.569030 & 9.685169 \\
\hline 8 & 6 & 0 & 3.613290 & 1.457068 & 9.989698 \\
\hline 9 & 6 & 0 & 2.707227 & 1.464367 & 11.147207 \\
\hline 10 & 8 & 0 & 2.544175 & 2.667349 & 11.792104 \\
\hline 11 & 8 & & 2.099405 & 0.506182 & 11.55887 \\
\hline 12 & 1 & ( & 1.960321 & 4.988197 & 12.73846 \\
\hline 13 & 1 & 0 & 2.963151 & 7.141064 & 11.99330 \\
\hline 14 & 1 & 0 & 4.616904 & 7.180810 & 10.14218 \\
\hline 15 & 1 & 0 & 5.284324 & 5.063907 & 9.033116 \\
\hline 16 & 1 & c & 5.146712 & 2.518864 & 8.939048 \\
\hline 17 & 1 & & 3.924409 & 0.471860 & 9.666117 \\
\hline 18 & 6 & U & 1.437735 & 0.369246 & 8.521988 \\
\hline 19 & 6 & 0 & 1.876828 & 1.669331 & 8.726389 \\
\hline 20 & 6 & 0 & 1.135551 & 2.700901 & 9.028614 \\
\hline 21 & 6 & 0 & -0.244934 & 2.629853 & 9.145243 \\
\hline 22 & 6 & 0 & -0.764699 & 1.321764 & 9.060276 \\
\hline 23 & 6 & 0 & 0.048828 & 0.235224 & 8.712903 \\
\hline 24 & 1 & 0 & 2.059357 & -0.481405 & 8.268083 \\
\hline 25 & 1 & 0 & -0.897695 & 3.475588 & 9.336327 \\
\hline 26 & 1 & 0 & -1.817976 & 1.151017 & 9.265233 \\
\hline 27 & 1 & 0 & -0.399364 & -0.745895 & 8.58940 \\
\hline
\end{tabular}




\section{Coumarin cycloaddition adduct (8)}

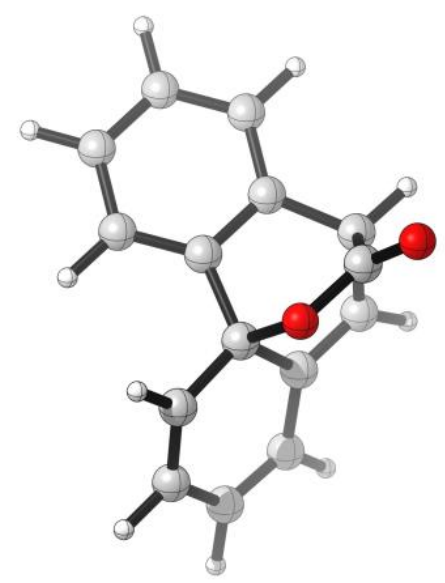

Zero-point correction $=$ 0.205556 (Hartree/Particle) Thermal correction to Gibbs Free Energy= 0.168275

Sum of electronic and thermal Free Energies $=\quad-727.946963$

\begin{tabular}{|c|c|c|c|c|}
\hline \multirow{2}{*}{$\begin{array}{l}\text { Center } \\
\text { Number }\end{array}$} & Atomic & \multirow{2}{*}{$\begin{array}{l}\text { Atomic } \\
\text { Type }\end{array}$} & \multicolumn{2}{|c|}{ Coordinates (Angstrom } \\
\hline & Number & & $X$ & $\mathrm{Z}$ \\
\hline 1 & 6 & -1.915767 & -0.451640 & -1.345726 \\
\hline 2 & 6 & -2.910071 & -1.073657 & -0.678614 \\
\hline 3 & 6 & -2.929772 & -1.091990 & 0.781071 \\
\hline 4 & 6 & -2.191280 & -0.212875 & 1.487896 \\
\hline 5 & 6 & -1.345220 & 0.815873 & 0.760139 \\
\hline 6 & 6 & -0.795849 & 0.241862 & -0.586835 \\
\hline 7 & 6 & -0.149429 & 1.418559 & 1.510243 \\
\hline 8 & 6 & 1.023221 & 1.415782 & 0.825814 \\
\hline 9 & 6 & 0.780060 & 2.087461 & -0.500937 \\
\hline 10 & 8 & -0.258695 & 1.436164 & -1.205999 \\
\hline 11 & 8 & 1.382083 & 3.078078 & -0.908275 \\
\hline 12 & 6 & 0.606858 & -1.989702 & -0.686533 \\
\hline 13 & 6 & 0.410713 & -0.655879 & -0.276768 \\
\hline 14 & 6 & 1.405946 & 0.036559 & 0.440912 \\
\hline 15 & 6 & 2.636312 & -0.584844 & 0.727897 \\
\hline 16 & 6 & 2.841465 & -1.919992 & 0.318666 \\
\hline 17 & 6 & 1.830685 & -2.620567 & -0.377390 \\
\hline 18 & 1 & -1.888794 & -0.421650 & -2.424942 \\
\hline 19 & 1 & -3.706326 & -1.552375 & -1.230436 \\
\hline 20 & 1 & -3.533595 & -1.823757 & 1.299433 \\
\hline 21 & 1 & -2.197948 & -0.207761 & 2.568186 \\
\hline 22 & 1 & -2.029717 & 1.636968 & 0.535204 \\
\hline 23 & 1 & -0.449005 & 2.385880 & 1.888426 \\
\hline 24 & 1 & -0.155381 & -2.517305 & -1.240553 \\
\hline 25 & 1 & 3.406856 & -0.051649 & 1.266011 \\
\hline 26 & 1 & 3.778551 & -2.410102 & 0.540727 \\
\hline 27 & 1 & 2.001022 & -3.641888 & -0.686888 \\
\hline
\end{tabular}




\section{Phenanthrene (9)}

Zero-point correction=

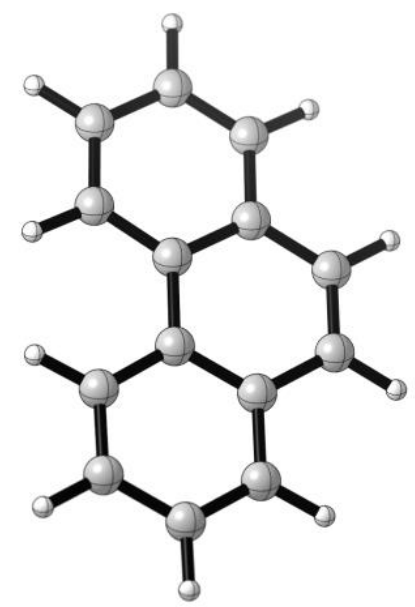

Thermal correction to Gibbs Free Energy=

0.193443 (Hartree/Particle)

Sum of electronic and thermal Free Energies $=\quad-539.528042$

\begin{tabular}{|c|c|c|c|c|}
\hline \multirow{2}{*}{$\begin{array}{l}\text { Center } \\
\text { Number }\end{array}$} & Atomic & \multirow{2}{*}{$\begin{array}{l}\text { Atomic } \\
\text { Type }\end{array}$} & \multicolumn{2}{|c|}{ Coordinates (Angstrom } \\
\hline & Number & & $\begin{array}{ll}X & Y\end{array}$ & $\mathrm{Z}$ \\
\hline 1 & 6 & -2.877773 & -1.529666 & 0.000036 \\
\hline 2 & 6 & -3.548777 & -0.314210 & 0.000007 \\
\hline 3 & 6 & -2.838149 & 0.881034 & -0.000024 \\
\hline 4 & 6 & -1.420541 & 0.875971 & 0.000008 \\
\hline 5 & 6 & -0.714663 & -0.368447 & -0.000011 \\
\hline 6 & 6 & -1.485924 & -1.572112 & 0.000020 \\
\hline 7 & 6 & -0.696758 & 2.086945 & -0.000031 \\
\hline 8 & 6 & 0.691254 & 2.087914 & 0.000002 \\
\hline 9 & 6 & 1.415748 & 0.877650 & 0.000048 \\
\hline 10 & 6 & 0.714012 & -0.365503 & -0.000029 \\
\hline 11 & 6 & 2.831207 & 0.878300 & 0.000036 \\
\hline 12 & 6 & 3.551230 & -0.309549 & -0.000016 \\
\hline 13 & 6 & 2.884483 & -1.524084 & -0.000022 \\
\hline 14 & 6 & 1.493978 & -1.561273 & -0.000030 \\
\hline 15 & 1 & -3.443914 & -2.451774 & -0.000013 \\
\hline 16 & 1 & -4.630602 & -0.298726 & -0.000024 \\
\hline 17 & 1 & -3.368335 & 1.824199 & 0.000014 \\
\hline 18 & 1 & -1.013493 & -2.541470 & -0.000028 \\
\hline 19 & 1 & -1.233505 & 3.026319 & -0.000037 \\
\hline 20 & 1 & 1.227198 & 3.027773 & 0.000013 \\
\hline 21 & 1 & 3.359401 & 1.822622 & 0.000024 \\
\hline 22 & 1 & 4.632972 & -0.288360 & 0.000016 \\
\hline 23 & 1 & 3.450116 & -2.446493 & 0.000040 \\
\hline 24 & 1 & 1.024200 & -2.531915 & 0.000026 \\
\hline
\end{tabular}




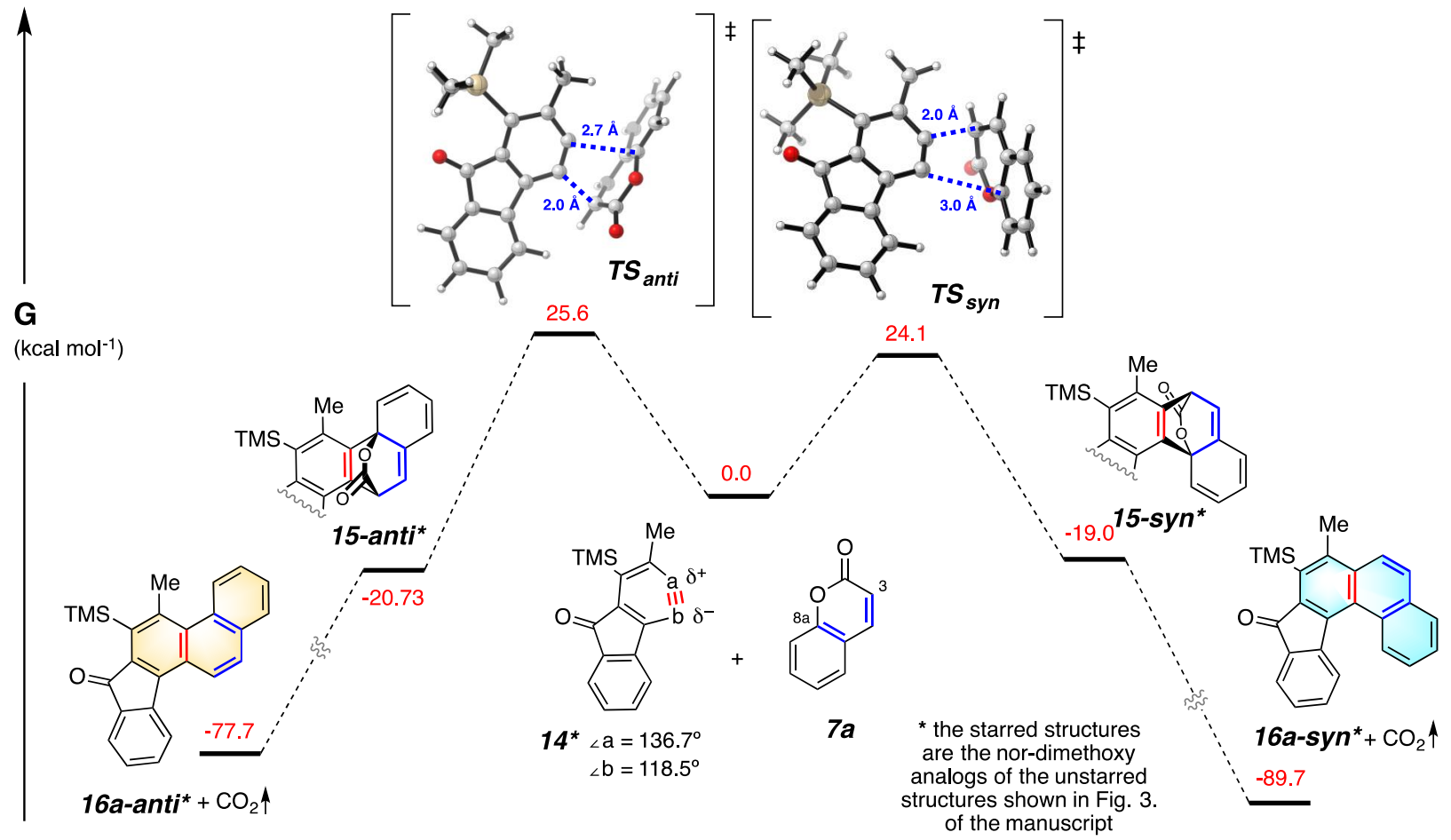

Supplementary Figure 2. DFT calculations $\left[\mathrm{SMD}\left(\mathrm{CHCl}_{3}\right) / \mathrm{B} 3 \mathrm{LYP} / 6-311 \mathrm{G}+(\mathrm{d}, \mathrm{p})\right.$ at $\left.358 \mathrm{~K}\right]$ for the reaction of benzyne 14 with coumarin (7a). (cf. Figure 4 in the manuscript)

HDDA benzyne (14*)

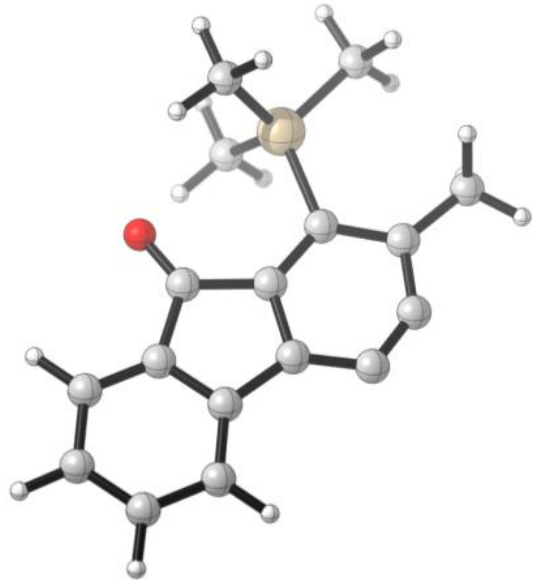

Zero-point correction=

0.272626 (Hartree/Particle)

Thermal correction to Gibbs Free Energy= 0.228266

Sum of electronic and thermal Free Energies $=\quad-1022.089262$

Center Atomic Atomic Coordinates (Angstroms)

Number Number Type $\quad X \quad$ Y $\quad$ Z

$\begin{array}{cccccc}1 & 6 & 0 & -4.721144 & -1.046050 & -0.000447 \\ 2 & 6 & 0 & -4.903547 & 0.340818 & -0.000107\end{array}$




\begin{tabular}{|c|c|c|c|c|c|}
\hline 3 & 6 & 0 & -3.796831 & 1.194619 & 0.000271 \\
\hline 4 & 6 & 0 & -2.512427 & 0.648704 & 0.000214 \\
\hline 5 & 6 & 0 & -2.332052 & -0.723938 & -0.000030 \\
\hline 6 & 6 & 0 & -3.431923 & -1.585984 & -0.000307 \\
\hline 7 & 6 & 0 & -0.887349 & -1.019141 & 0.000101 \\
\hline 8 & 6 & 0 & -0.222682 & 0.290996 & 0.000154 \\
\hline 9 & 6 & 0 & -1.191273 & 1.257702 & 0.000311 \\
\hline 10 & 6 & 0 & -0.660993 & 2.613859 & 0.000384 \\
\hline 11 & 6 & 0 & 0.535940 & 2.900282 & 0.000005 \\
\hline 12 & 6 & 0 & 1.636982 & 1.937227 & -0.000185 \\
\hline 13 & 6 & 0 & 1.244536 & 0.610068 & -0.000000 \\
\hline 14 & 8 & 0 & -0.345172 & -2.120202 & 0.000088 \\
\hline 15 & 14 & 0 & 2.568224 & -0.810787 & 0.000088 \\
\hline 16 & 6 & 0 & 3.046418 & 2.501039 & -0.000697 \\
\hline 17 & 6 & 0 & 2.441449 & -1.881351 & 1.544432 \\
\hline 18 & 6 & 0 & 2.441332 & -1.882064 & -1.543868 \\
\hline 19 & 6 & 0 & 4.387621 & -0.318914 & -0.000204 \\
\hline 20 & 1 & 0 & -5.578955 & -1.702898 & -0.000744 \\
\hline 21 & 1 & 0 & -5.901384 & 0.754983 & -0.000082 \\
\hline 22 & 1 & 0 & -3.940458 & 2.265834 & 0.000493 \\
\hline 23 & 1 & 0 & -3.299140 & -2.659633 & -0.000626 \\
\hline 24 & 1 & 0 & 3.047319 & 3.592729 & -0.001116 \\
\hline 25 & 1 & 0 & 3.584129 & 2.191837 & 0.893261 \\
\hline 26 & 1 & 0 & 3.583751 & 2.191101 & -0.894639 \\
\hline 27 & 1 & 0 & 3.199713 & -2.663054 & 1.539274 \\
\hline 28 & 1 & 0 & 2.590627 & -1.284311 & 2.443121 \\
\hline 29 & 1 & 0 & 1.476361 & -2.372498 & 1.640913 \\
\hline 30 & 1 & 0 & 3.199818 & -2.663560 & -1.538426 \\
\hline 31 & 1 & 0 & 1.476396 & -2.373629 & -1.639886 \\
\hline 32 & 1 & 0 & 2.590146 & -1.285340 & -2.442779 \\
\hline 33 & 1 & 0 & 5.016780 & -1.208333 & -0.000099 \\
\hline 34 & 1 & 0 & 4.658288 & 0.250860 & -0.886937 \\
\hline 35 & 1 & 0 & 4.658511 & 0.251315 & 0.886163 \\
\hline
\end{tabular}




\section{Reaction of HDDA benzyne and coumarin (TS anti)}

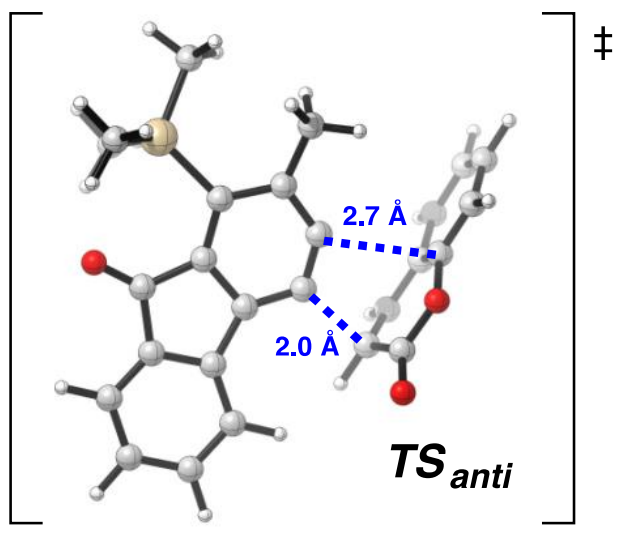

Zero-point correction= 0.400139 (Hartree/Particle)

Thermal correction to Gibbs Free Energy $=0.342058$

Sum of electronic and thermal Free Energies $=\quad-1519.127304$

\begin{tabular}{|c|c|c|c|c|}
\hline \multirow{2}{*}{$\begin{array}{l}\text { Center } \\
\text { Number }\end{array}$} & Atomic & \multirow{2}{*}{$\begin{array}{l}\text { Atomic } \\
\text { Type }\end{array}$} & \multicolumn{2}{|c|}{ Coordinates (Angstroms } \\
\hline & Number & & $\begin{array}{ll}X & Y\end{array}$ & $\mathrm{Z}$ \\
\hline 1 & 6 & -3.620335 & 4.167528 & 0.270157 \\
\hline 2 & 6 & -2.283654 & 4.560377 & 0.151647 \\
\hline 3 & 6 & -1.247660 & 3.615346 & 0.060223 \\
\hline 4 & 6 & -1.577279 & 2.262972 & 0.104095 \\
\hline 5 & 6 & -2.927895 & 1.877014 & 0.194395 \\
\hline 6 & 6 & -3.955341 & 2.805082 & 0.280100 \\
\hline 7 & 6 & -3.018184 & 0.387280 & 0.115542 \\
\hline 8 & 6 & -1.606100 & -0.124227 & -0.008910 \\
\hline 9 & 6 & -0.760876 & 1.032187 & 0.022129 \\
\hline 10 & 6 & 0.586411 & 0.739526 & -0.066487 \\
\hline 11 & 6 & 1.003841 & -0.459667 & -0.303012 \\
\hline 12 & 6 & 0.264916 & -1.622411 & -0.460961 \\
\hline 13 & 6 & -1.145158 & -1.447192 & -0.201397 \\
\hline 14 & 8 & -4.056008 & -0.258808 & 0.115986 \\
\hline 15 & 14 & -2.386254 & -2.935635 & -0.041576 \\
\hline 16 & 6 & 0.924865 & -2.910760 & -0.894973 \\
\hline 17 & 6 & -3.250077 & -2.846706 & 1.641725 \\
\hline 18 & 6 & -3.573629 & -2.962295 & -1.512526 \\
\hline 19 & 6 & -1.545891 & -4.641712 & -0.011831 \\
\hline 20 & 1 & -4.400253 & 4.919902 & 0.336894 \\
\hline 21 & 1 & -2.038255 & 5.617967 & 0.116242 \\
\hline 22 & 1 & -0.226066 & 3.950268 & -0.081463 \\
\hline 23 & 1 & -4.987675 & 2.473930 & 0.343088 \\
\hline 24 & 1 & 1.933507 & -2.693672 & -1.251713 \\
\hline 25 & 1 & 1.011351 & -3.630665 & -0.075291 \\
\hline 26 & 1 & 0.375113 & -3.397617 & -1.704475 \\
\hline 27 & 1 & -3.904422 & -3.720424 & 1.752961 \\
\hline 28 & 1 & -2.511157 & -2.886541 & 2.451170 \\
\hline
\end{tabular}




$\begin{array}{rrrrrr}29 & 1 & 0 & -3.858969 & -1.950327 & 1.764190 \\ 30 & 1 & 0 & -3.022606 & -3.079555 & -2.453130 \\ 31 & 1 & 0 & -4.255304 & -3.816833 & -1.419750 \\ 32 & 1 & 0 & -4.173201 & -2.051730 & -1.569185 \\ 33 & 1 & 0 & -2.339960 & -5.365624 & 0.212868 \\ 34 & 1 & 0 & -1.094255 & -4.942735 & -0.960548 \\ 35 & 1 & 0 & -0.793162 & -4.739374 & 0.777085 \\ 36 & 6 & 0 & 4.420973 & -0.858942 & -0.959382 \\ 37 & 6 & 0 & 4.961437 & -1.809870 & -0.112388 \\ 38 & 6 & 0 & 4.808314 & -1.705663 & 1.290647 \\ 39 & 6 & 0 & 4.116582 & -0.643132 & 1.834530 \\ 40 & 6 & 0 & 3.549090 & 0.358400 & 0.999791 \\ 41 & 6 & 0 & 3.695272 & 0.212998 & -0.409679 \\ 42 & 6 & 0 & 2.767844 & 1.436837 & 1.460587 \\ 43 & 6 & 0 & 2.041607 & 2.161976 & 0.519750 \\ 44 & 6 & 0 & 2.561950 & 2.278743 & -0.854590 \\ 45 & 8 & 0 & 3.364176 & 1.228249 & -1.265859 \\ 46 & 8 & 0 & 2.346660 & 3.184972 & -1.623939 \\ 47 & 1 & 0 & 4.548129 & -0.906644 & -2.035400 \\ 48 & 1 & 0 & 5.515009 & -2.645082 & -0.530670 \\ 49 & 1 & 0 & 5.239371 & -2.463693 & 1.936727 \\ 50 & 1 & 0 & 3.996336 & -0.551459 & 2.910305 \\ 51 & 1 & 0 & 2.581171 & 1.565744 & 2.522453 \\ 52 & 1 & 0 & 1.428088 & 2.995623 & 0.835108 \\ --------------------------------------------------------------\end{array}$




\section{Reaction adduct (15-anti*)}

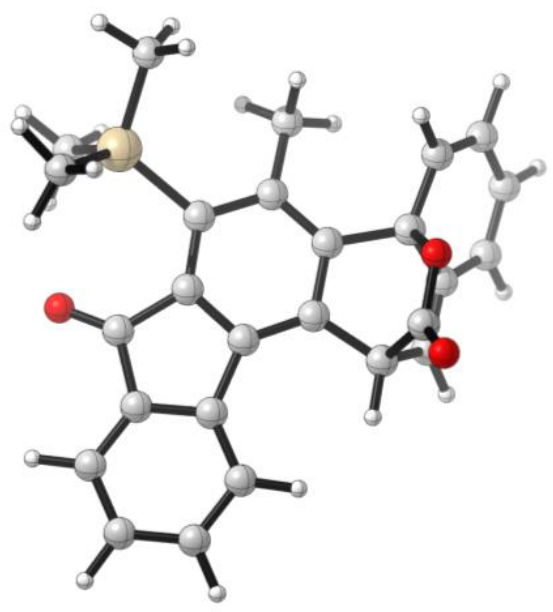

Zero-point correction= 0.405009 (Hartree/Particle)

Thermal correction to Gibbs Free Energy $=\quad 0.350232$

Sum of electronic and thermal Free Energies $=\quad-1519.201095$

\begin{tabular}{|c|c|c|c|c|c|}
\hline \multirow{3}{*}{$\begin{array}{l}\text { Center } \\
\text { Number } \\
1\end{array}$} & \multirow{2}{*}{\multicolumn{2}{|c|}{$\begin{array}{l}\text { Atomic } \\
\text { Number }\end{array}$}} & & \multicolumn{2}{|c|}{ Coordinates (Angstroms } \\
\hline & & & & X $\quad \mathrm{Y}$ & $\mathrm{Z}$ \\
\hline & 6 & & & -3.866924 & -0.426586 \\
\hline 2 & 6 & 0 & 2.674045 & -4.410971 & -0.288957 \\
\hline 3 & 6 & 0 & 1.563740 & -3.573018 & -0.161310 \\
\hline 4 & 6 & 0 & 1.720252 & -2.179478 & -0.169076 \\
\hline 5 & 6 & 0 & 2.999211 & -1.656437 & -0.302080 \\
\hline 6 & 6 & 0 & 4.118262 & -2.483293 & -0.434481 \\
\hline 7 & 6 & 0 & 2.947771 & -0.190142 & -0.283812 \\
\hline 8 & 6 & 0 & 1.506003 & 0.154073 & -0.097969 \\
\hline 9 & 6 & 0 & 0.774638 & -1.040860 & -0.060768 \\
\hline 10 & 6 & 0 & -0.618072 & -0.996640 & 0.079382 \\
\hline 11 & 6 & 0 & -1.281310 & 0.255384 & 0.140865 \\
\hline 12 & 6 & 0 & -0.538911 & 1.445520 & 0.004541 \\
\hline 13 & 6 & 0 & 0.873599 & 1.417724 & 0.001285 \\
\hline 14 & 8 & 0 & 3.915429 & 0.554888 & -0.416919 \\
\hline 15 & 14 & 0 & 1.974522 & 3.007687 & 0.173351 \\
\hline 16 & 6 & 0 & 1.102592 & 4.609114 & 0.667595 \\
\hline 17 & 6 & 0 & 3.208894 & 2.876142 & 1.593926 \\
\hline 18 & 6 & 0 & 2.871778 & 3.440805 & -1.422620 \\
\hline 19 & 6 & 0 & -1.264408 & 2.762916 & -0.224930 \\
\hline 20 & 6 & 0 & -1.568573 & -2.228733 & 0.150390 \\
\hline 21 & 6 & 0 & -2.596056 & -1.976049 & -0.980144 \\
\hline 22 & 6 & 0 & -3.289219 & -0.815922 & -0.881974 \\
\hline 23 & 6 & 0 & -2.823373 & 0.046331 & 0.333046 \\
\hline 24 & 6 & 0 & -4.362245 & -0.389581 & -1.766798 \\
\hline 25 & 6 & 0 & -5.055205 & 0.734754 & -1.507062 \\
\hline 26 & 6 & 0 & -4.772129 & 1.547312 & -0.334626 \\
\hline 27 & 6 & 0 & -3.762619 & 1.250070 & 0.513499 \\
\hline
\end{tabular}




$\begin{array}{rrrrrr}28 & 8 & 0 & -2.989889 & -0.793160 & 1.517218 \\ 29 & 6 & 0 & -2.311205 & -2.014605 & 1.498181 \\ 30 & 8 & 0 & -2.392693 & -2.770631 & 2.462958 \\ 31 & 1 & 0 & 4.809513 & -4.515473 & -0.525101 \\ 32 & 1 & 0 & 2.543267 & -5.483301 & -0.280806 \\ 33 & 1 & 0 & 0.607734 & -4.050257 & -0.057516 \\ 34 & 1 & 0 & 5.110280 & -2.063994 & -0.539669 \\ 35 & 1 & 0 & 1.826215 & 5.347201 & 1.013522 \\ 36 & 1 & 0 & 0.574574 & 5.072237 & -0.163761 \\ 37 & 1 & 0 & 0.399727 & 4.448437 & 1.483419 \\ 38 & 1 & 0 & 3.840107 & 3.762510 & 1.640380 \\ 39 & 1 & 0 & 2.688798 & 2.796642 & 2.547841 \\ 40 & 1 & 0 & 3.871955 & 2.018977 & 1.521764 \\ 41 & 1 & 0 & 3.496031 & 4.323636 & -1.292487 \\ 42 & 1 & 0 & 3.518168 & 2.635688 & -1.765866 \\ 43 & 1 & 0 & 2.163736 & 3.651265 & -2.223005 \\ 44 & 1 & 0 & -0.671775 & 3.435907 & -0.837056 \\ 45 & 1 & 0 & -2.158061 & 2.621775 & -0.827904 \\ 46 & 1 & 0 & -1.529988 & 3.247367 & 0.714372 \\ 47 & 1 & 0 & -1.130424 & -3.215593 & 0.136156 \\ 48 & 1 & 0 & -2.794991 & -2.697690 & -1.760118 \\ 49 & 1 & 0 & -4.604587 & -0.978941 & -2.639912 \\ 50 & 1 & 0 & -5.846662 & 1.036042 & -2.179554 \\ 51 & 1 & 0 & -5.405226 & 2.398134 & -0.121880 \\ 52 & 1 & 0 & -3.623052 & 1.868092 & 1.387118 \\ ---------------------------------------------------------\end{array}$




\section{HDDA product (16a-anti*)}

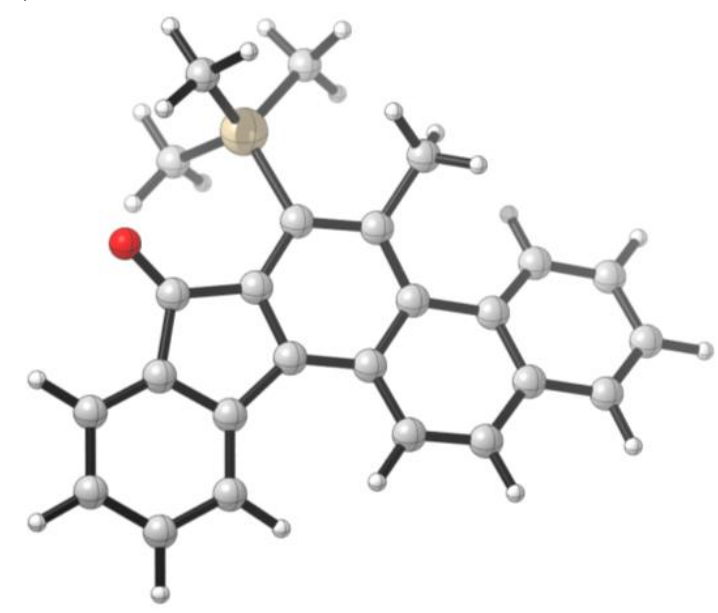

Zero-point correction $=\quad 0.390925$ (Hartree/Particle)

Thermal correction to Gibbs Free Energy $=0.338177$

Sum of electronic and thermal Free Energies $=\quad-1330.637550$

\begin{tabular}{|c|c|c|c|c|c|}
\hline \multirow{3}{*}{$\begin{array}{l}\text { Center } \\
\text { Number } \\
-1\end{array}$} & \multirow{2}{*}{\multicolumn{2}{|c|}{$\begin{array}{l}\text { Atomic } \\
\text { Number }\end{array}$}} & \multirow{2}{*}{$\begin{array}{l}\text { Atomic } \\
\text { Type }\end{array}$} & \multicolumn{2}{|c|}{ Coordinates (Angstror } \\
\hline & & & & X $\quad Y$ & $\mathrm{Z}$ \\
\hline & 6 & 0 & -3.996839 & -3.818250 & 0.000428 \\
\hline 2 & 6 & 0 & -2.755019 & -4.445607 & 0.000228 \\
\hline 3 & 6 & 0 & -1.582541 & -3.686012 & 0.000128 \\
\hline 4 & 6 & 0 & -1.625342 & -2.275678 & 0.000128 \\
\hline 5 & 6 & 0 & -2.877934 & -1.684512 & 0.000528 \\
\hline 6 & 6 & 0 & -4.061290 & -2.429196 & 0.000628 \\
\hline 7 & 6 & 0 & -2.747284 & -0.234601 & 0.000828 \\
\hline 8 & 6 & 0 & -1.281010 & 0.057742 & 0.000328 \\
\hline 9 & 6 & 0 & -0.606088 & -1.170206 & 0.000028 \\
\hline 10 & 6 & 0 & 0.825747 & -1.192230 & -0.000472 \\
\hline 11 & 6 & 0 & 1.602839 & 0.022435 & -0.000172 \\
\hline 12 & 6 & 0 & 0.827638 & 1.266262 & 0.000128 \\
\hline 13 & 6 & 0 & -0.594503 & 1.292441 & 0.000228 \\
\hline 14 & 8 & 0 & -3.705936 & 0.522899 & 0.001528 \\
\hline 15 & 14 & & -1.567741 & 3.011150 & 0.000228 \\
\hline 16 & 6 & 0 & 1.559967 & 2.604831 & 0.000328 \\
\hline 17 & 6 & & -1.148562 & 4.008252 & 1.551528 \\
\hline 18 & 6 & 0 & -1.148250 & 4.008308 & -1.551072 \\
\hline 19 & 6 & 0 & -3.436739 & 3.243019 & -0.000272 \\
\hline 20 & 6 & 0 & 1.497627 & -2.432550 & -0.001172 \\
\hline 21 & 6 & 0 & 2.863641 & -2.544983 & -0.001372 \\
\hline 22 & 6 & 0 & 3.665068 & -1.410346 & -0.000972 \\
\hline 23 & 6 & 0 & 3.064727 & -0.102815 & -0.000272 \\
\hline 24 & 6 & 0 & 5.072070 & -1.598042 & -0.001172 \\
\hline 25 & 6 & 0 & 5.930721 & -0.514988 & -0.000772 \\
\hline 26 & 6 & 0 & 5.409342 & 0.762287 & -0.000072 \\
\hline 27 & 6 & 0 & 4.032864 & 0.970868 & 0.000128 \\
\hline 28 & 1 & 0 & -4.905150 & -4.403729 & 0.000528 \\
\hline
\end{tabular}




$\begin{array}{rrrrrr}29 & 1 & 0 & -2.698258 & -5.524728 & 0.000128 \\ 30 & 1 & 0 & -0.687812 & -4.274925 & 0.000028 \\ 31 & 1 & 0 & -5.026112 & -1.939976 & 0.000928 \\ 32 & 1 & 0 & 0.968738 & 3.502597 & 0.000328 \\ 33 & 1 & 0 & 2.150615 & 2.699167 & 0.911228 \\ 34 & 1 & 0 & 2.150878 & 2.699311 & -0.910372 \\ 35 & 1 & 0 & -1.620869 & 4.989533 & 1.519228 \\ 36 & 1 & 0 & -1.526248 & 3.500167 & 2.438428 \\ 37 & 1 & 0 & -0.085599 & 4.168875 & 1.711728 \\ 38 & 1 & 0 & -1.620470 & 4.989637 & -1.518872 \\ 39 & 1 & 0 & -0.085240 & 4.168844 & -1.711272 \\ 40 & 1 & 0 & -1.525849 & 3.500271 & -2.438072 \\ 41 & 1 & 0 & -3.692133 & 4.301968 & -0.000772 \\ 42 & 1 & 0 & -3.900946 & 2.815753 & -0.888272 \\ 43 & 1 & 0 & -3.901202 & 2.816638 & 0.888028 \\ 44 & 1 & 0 & 0.968823 & -3.353473 & -0.001672 \\ 45 & 1 & 0 & 3.319755 & -3.525195 & -0.001872 \\ 46 & 1 & 0 & 5.479271 & -2.599268 & -0.001672 \\ 47 & 1 & 0 & 7.000760 & -0.665886 & -0.000972 \\ 48 & 1 & 0 & 6.082749 & 1.607449 & 0.000228 \\ 49 & 1 & 0 & 3.805070 & 2.009112 & 0.000728 \\ ------------------------------------------------------------\end{array}$




\section{Reaction of HDDA benzyne and coumarin $\left(\mathrm{TS}_{\mathrm{syn}}\right)$}

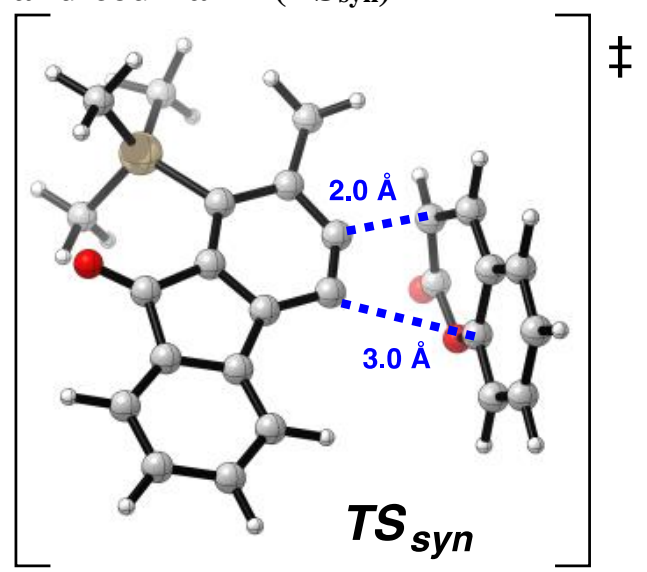

Zero-point correction $=\quad 0.400246($ Hartree/Particle $)$

Thermal correction to Gibbs Free Energy $=0.341436$

Sum of electronic and thermal Free Energies $=\quad-1519.129684$

\begin{tabular}{cccccc} 
Center & Atomic & \multicolumn{2}{c}{ Atomic } & \multicolumn{3}{c}{ Coordinates } \\
Number & Number & Type & X & Y & Z \\
\hline 1 & 6 & 0 & -0.522799 & 5.168671 & 0.312186 \\
2 & 6 & 0 & 0.766752 & 4.649644 & 0.444873 \\
3 & 6 & 0 & 1.006595 & 3.269411 & 0.384585 \\
4 & 6 & 0 & -0.075459 & 2.421793 & 0.193558 \\
5 & 6 & 0 & -1.370298 & 2.947889 & 0.062270 \\
6 & 6 & 0 & -1.612488 & 4.311637 & 0.113900 \\
7 & 6 & 0 & -2.328579 & 1.821088 & -0.150222 \\
8 & 6 & 0 & -1.515303 & 0.552009 & -0.073854 \\
9 & 6 & 0 & -0.160166 & 0.950935 & 0.083590 \\
10 & 6 & 0 & 0.794146 & -0.048912 & 0.047732 \\
11 & 6 & 0 & 0.395603 & -1.269663 & -0.130671 \\
12 & 6 & 0 & -0.885851 & -1.781583 & -0.358095 \\
13 & 6 & 0 & -1.918745 & -0.790778 & -0.241666 \\
14 & 8 & 0 & -3.515262 & 1.935511 & -0.397470 \\
15 & 14 & 0 & -3.806227 & -1.244810 & -0.115268 \\
16 & 6 & 0 & -1.143756 & -3.213468 & -0.750818 \\
17 & 6 & 0 & -4.523962 & -0.396646 & 1.413744 \\
18 & 6 & 0 & -4.707740 & -0.811691 & -1.713149 \\
19 & 6 & 0 & -4.135036 & -3.079746 & 0.235297 \\
20 & 1 & 0 & -0.679401 & 6.240189 & 0.361899 \\
21 & 1 & 0 & 1.599553 & 5.327984 & 0.596339 \\
22 & 1 & 0 & 2.012192 & 2.877623 & 0.484609 \\
23 & 1 & 0 & -2.618670 & 4.701309 & 0.005036 \\
24 & 1 & 0 & -0.349549 & -3.575237 & -1.406693 \\
25 & 1 & 0 & -1.193829 & -3.884680 & 0.112424 \\
26 & 1 & 0 & -2.073254 & -3.309656 & -1.305388 \\
27 & 1 & 0 & -5.578167 & -0.681307 & 1.511316 \\
28 & 1 & 0 & -4.008615 & -0.746915 & 2.315108 \\
29 & 1 & 0 & -4.474686 & 0.690816 & 1.388407
\end{tabular}




$\begin{array}{rrrrrr}30 & 1 & 0 & -5.769422 & -1.070200 & -1.626294 \\ 31 & 1 & 0 & -4.634452 & 0.251726 & -1.947167 \\ 32 & 1 & 0 & -4.298133 & -1.379861 & -2.555600 \\ 33 & 1 & 0 & -5.175963 & -3.147047 & 0.574302 \\ 34 & 1 & 0 & -4.039954 & -3.734675 & -0.633102 \\ 35 & 1 & 0 & -3.511103 & -3.481054 & 1.039041 \\ 36 & 6 & 0 & 4.326088 & 0.536067 & 0.724974 \\ 37 & 6 & 0 & 4.982115 & 1.162379 & -0.318247 \\ 38 & 6 & 0 & 4.923621 & 0.648248 & -1.631135 \\ 39 & 6 & 0 & 4.209383 & -0.498923 & -1.890509 \\ 40 & 6 & 0 & 3.521501 & -1.170847 & -0.846319 \\ 41 & 6 & 0 & 3.577495 & -0.614649 & 0.458139 \\ 42 & 6 & 0 & 2.710200 & -2.301683 & -1.035984 \\ 43 & 6 & 0 & 1.867691 & -2.694488 & 0.003172 \\ 44 & 6 & 0 & 2.262003 & -2.383136 & 1.387785 \\ 45 & 8 & 0 & 3.093224 & -1.301336 & 1.542113 \\ 46 & 8 & 0 & 1.917920 & -2.985750 & 2.374529 \\ 47 & 1 & 0 & 4.379240 & 0.909365 & 1.740167 \\ 48 & 1 & 0 & 5.555113 & 2.061407 & -0.121383 \\ 49 & 1 & 0 & 5.445018 & 1.159170 & -2.431675 \\ 50 & 1 & 0 & 4.156202 & -0.907573 & -2.893419 \\ 51 & 1 & 0 & 2.620647 & -2.754000 & -2.017177 \\ 52 & 1 & 0 & 1.299288 & -3.608795 & -0.081189 \\ ---------------------------------------------------------------\end{array}$




\section{Reaction adduct (15-syn*)}

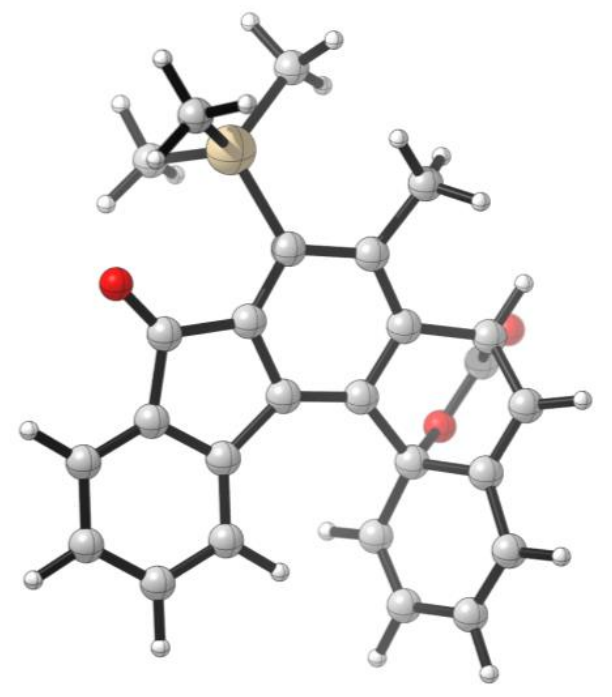

Zero-point correction= 0.404645 (Hartree/Particle)

Thermal correction to Gibbs Free Energy= 0.349324

Sum of electronic and thermal Free Energies $=\quad-1519.198393$

\begin{tabular}{ccccccc} 
Center & Atomic & \multicolumn{2}{c}{ Atomic } & \multicolumn{3}{c}{ Coordinates } \\
Number & Number & Type & X & Y & Z \\
- & Nutroms) \\
\hline 1 & 6 & 0 & 0.422580 & 5.015850 & 0.224915 \\
2 & 6 & 0 & 1.600906 & 4.349663 & -0.111380 \\
3 & 6 & 0 & 1.606535 & 2.957506 & -0.231394 \\
4 & 6 & 0 & 0.450635 & 2.210594 & 0.026724 \\
5 & 6 & 0 & -0.729834 & 2.901175 & 0.255697 \\
6 & 6 & 0 & -0.757510 & 4.292532 & 0.389021 \\
7 & 6 & 0 & -1.859810 & 1.970761 & 0.227501 \\
8 & 6 & 0 & -1.257361 & 0.620411 & 0.005787 \\
9 & 6 & 0 & 0.141923 & 0.756697 & 0.031253 \\
10 & 6 & 0 & 0.940774 & -0.401767 & 0.078754 \\
11 & 6 & 0 & 0.334337 & -1.663718 & -0.148312 \\
12 & 6 & 0 & -1.051290 & -1.761931 & -0.344918 \\
13 & 6 & 0 & -1.886649 & -0.637751 & -0.174958 \\
14 & 8 & 0 & -3.039890 & 2.293426 & 0.336109 \\
15 & 14 & 0 & -3.828815 & -0.732177 & -0.177015 \\
16 & 6 & 0 & -4.656403 & -2.415766 & -0.378524 \\
17 & 6 & 0 & -4.577186 & -0.195552 & 1.470412 \\
18 & 6 & 0 & -4.581925 & 0.258876 & -1.591614 \\
19 & 6 & 0 & -1.637998 & -3.115799 & -0.722547 \\
20 & 6 & 0 & 2.471898 & -0.584515 & 0.358879 \\
21 & 6 & 0 & 3.062171 & -1.320067 & -0.886891 \\
22 & 6 & 0 & 2.419787 & -2.483817 & -1.147183 \\
23 & 6 & 0 & 1.323314 & -2.871454 & -0.127898 \\
24 & 6 & 0 & 3.363444 & 0.602418 & 0.740247 \\
& & & & &
\end{tabular}




$\begin{array}{rrrrrr}25 & 6 & 0 & 4.465145 & 0.966722 & 0.043695 \\ 26 & 6 & 0 & 4.870985 & 0.277193 & -1.170727 \\ 27 & 6 & 0 & 4.211157 & -0.807932 & -1.615837 \\ 28 & 8 & 0 & 2.583562 & -1.528404 & 1.469662 \\ 29 & 6 & 0 & 1.956429 & -2.762981 & 1.286927 \\ 30 & 8 & 0 & 1.992599 & -3.602636 & 2.182836 \\ 31 & 1 & 0 & 0.412529 & 6.092941 & 0.313783 \\ 32 & 1 & 0 & 2.504977 & 4.911571 & -0.296306 \\ 33 & 1 & 0 & 2.512957 & 2.493151 & -0.567991 \\ 34 & 1 & 0 & -1.685548 & 4.815160 & 0.581043 \\ 35 & 1 & 0 & -5.729123 & -2.320793 & -0.219351 \\ 36 & 1 & 0 & -4.533817 & -2.827613 & -1.378414 \\ 37 & 1 & 0 & -4.297623 & -3.141402 & 0.349211 \\ 38 & 1 & 0 & -5.664853 & -0.168717 & 1.413625 \\ 39 & 1 & 0 & -4.310445 & -0.894319 & 2.262344 \\ 40 & 1 & 0 & -4.251503 & 0.788301 & 1.796244 \\ 41 & 1 & 0 & -5.670146 & 0.228403 & -1.555932 \\ 42 & 1 & 0 & -4.288860 & 1.305849 & -1.574640 \\ 43 & 1 & 0 & -4.274703 & -0.144750 & -2.555498 \\ 44 & 1 & 0 & -2.376998 & -2.997909 & -1.510415 \\ 45 & 1 & 0 & -0.909101 & -3.797245 & -1.153770 \\ 46 & 1 & 0 & -2.082462 & -3.602190 & 0.145464 \\ 47 & 1 & 0 & 2.717971 & -3.132923 & -1.957773 \\ 48 & 1 & 0 & 0.913680 & -3.869840 & -0.250232 \\ 49 & 1 & 0 & 3.122883 & 1.139255 & 1.645612 \\ 50 & 1 & 0 & 5.064221 & 1.792422 & 0.401689 \\ 51 & 1 & 0 & 5.721799 & 0.643372 & -1.727930 \\ 52 & 1 & 0 & 4.541087 & -1.305408 & -2.517187 \\ -------------------------------------------------------\end{array}$




\section{HDDA product (16a-syn*)}

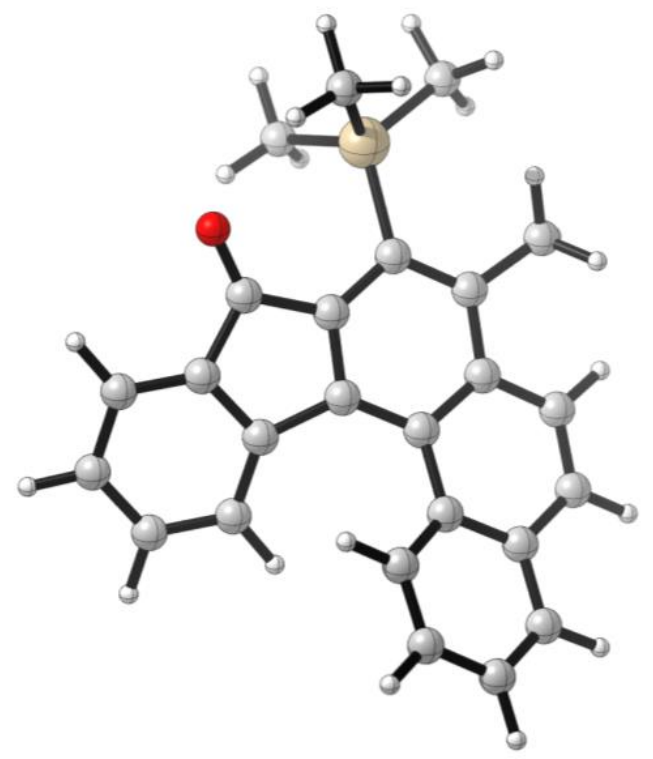

Zero-point correction $=\quad 0.390958$ (Hartree/Particle)

Thermal correction to Gibbs Free Energy= 0.338298

Sum of electronic and thermal Free Energies $=\quad-1330.656736$

\begin{tabular}{|c|c|c|c|c|c|}
\hline \multirow{2}{*}{$\begin{array}{l}\text { Center } \\
\text { Number }\end{array}$} & Atomic & \multirow{2}{*}{\multicolumn{2}{|c|}{$\begin{array}{l}\text { Atomic } \\
\text { Type }\end{array}$}} & \multicolumn{2}{|c|}{ Coordinates (Angstroms } \\
\hline & Numbe & & & $\begin{array}{ll}\mathrm{X} & \mathrm{Y}\end{array}$ & $\mathrm{Z}$ \\
\hline 1 & 6 & 0 & -1.016563 & 4.689370 & -0.373481 \\
\hline 2 & 6 & 0 & -2.050198 & 3.888017 & -0.862511 \\
\hline 3 & 6 & 0 & -1.939123 & 2.496085 & -0.813568 \\
\hline 4 & 6 & 0 & -0.805300 & 1.896648 & -0.248848 \\
\hline 5 & 6 & 0 & 0.248759 & 2.704977 & 0.144712 \\
\hline 6 & 6 & 0 & 0.145165 & 4.098693 & 0.125335 \\
\hline 7 & 6 & 0 & 1.405712 & 1.865186 & 0.476020 \\
\hline 8 & 6 & 0 & 0.974546 & 0.471711 & 0.152634 \\
\hline 9 & 6 & 0 & -0.413306 & 0.482364 & -0.020883 \\
\hline 10 & 6 & 0 & -1.149993 & -0.751022 & -0.012340 \\
\hline 11 & 6 & 0 & -0.403011 & -1.963912 & -0.148651 \\
\hline 12 & 6 & 0 & 1.025639 & -1.931285 & -0.032282 \\
\hline 13 & 6 & 0 & 1.727020 & -0.716229 & 0.076954 \\
\hline 14 & 8 & 0 & 2.487570 & 2.262767 & 0.901263 \\
\hline 15 & 14 & 0 & 3.662588 & -0.623886 & -0.014906 \\
\hline 16 & 6 & 0 & 1.773368 & -3.264557 & 0.033968 \\
\hline 17 & 6 & 0 & 4.435910 & -0.746626 & 1.689662 \\
\hline 18 & 6 & 0 & 4.437269 & -1.987208 & -1.067998 \\
\hline 19 & 6 & 0 & 4.374196 & 0.895840 & -0.877575 \\
\hline 20 & 6 & 0 & -2.571654 & -0.835751 & 0.146944 \\
\hline 21 & 6 & 0 & -3.252936 & -2.061257 & -0.131725 \\
\hline 22 & 6 & 0 & -2.500953 & -3.201337 & -0.455728 \\
\hline 23 & 6 & 0 & -1.117327 & -3.166599 & -0.410494 \\
\hline 24 & 6 & 0 & -3.358905 & 0.233298 & 0.670640 \\
\hline
\end{tabular}




$\begin{array}{rrrrrr}25 & 6 & 0 & -4.744141 & 0.137328 & 0.768993 \\ 26 & 6 & 0 & -5.397615 & -1.026911 & 0.389531 \\ 27 & 6 & 0 & -4.666739 & -2.127404 & -0.044509 \\ 28 & 1 & 0 & -1.101330 & 5.766444 & -0.411942 \\ 29 & 1 & 0 & -2.934532 & 4.343608 & -1.283949 \\ 30 & 1 & 0 & -2.740121 & 1.895571 & -1.219793 \\ 31 & 1 & 0 & 0.967547 & 4.722611 & 0.449540 \\ 32 & 1 & 0 & 2.674848 & -3.184155 & 0.637214 \\ 33 & 1 & 0 & 1.197152 & -4.035086 & 0.544513 \\ 34 & 1 & 0 & 2.031264 & -3.620052 & -0.963936 \\ 35 & 1 & 0 & 5.523598 & -0.724792 & 1.635597 \\ 36 & 1 & 0 & 4.117678 & 0.084800 & 2.317455 \\ 37 & 1 & 0 & 4.145659 & -1.670355 & 2.189409 \\ 38 & 1 & 0 & 5.460924 & -1.732477 & -1.341466 \\ 39 & 1 & 0 & 4.489786 & -2.944740 & -0.555372 \\ 40 & 1 & 0 & 3.879961 & -2.123842 & -1.994219 \\ 41 & 1 & 0 & 5.290138 & 0.659907 & -1.416879 \\ 42 & 1 & 0 & 3.668251 & 1.310540 & -1.595674 \\ 43 & 1 & 0 & 4.634424 & 1.678012 & -0.165440 \\ 44 & 1 & 0 & -3.010920 & -4.130946 & -0.666779 \\ 45 & 1 & 0 & -0.587643 & -4.085716 & -0.607376 \\ 46 & 1 & 0 & -2.881162 & 1.121752 & 1.049587 \\ 47 & 1 & 0 & -5.315383 & 0.967657 & 1.159845 \\ 48 & 1 & 0 & -6.472859 & -1.091554 & 0.473399 \\ 49 & 1 & 0 & -5.172993 & -3.051427 & -0.283949 \\ ---------------------------------------------------------\end{array}$


NBO (Natural Bond Orbital) analysis of coumarin

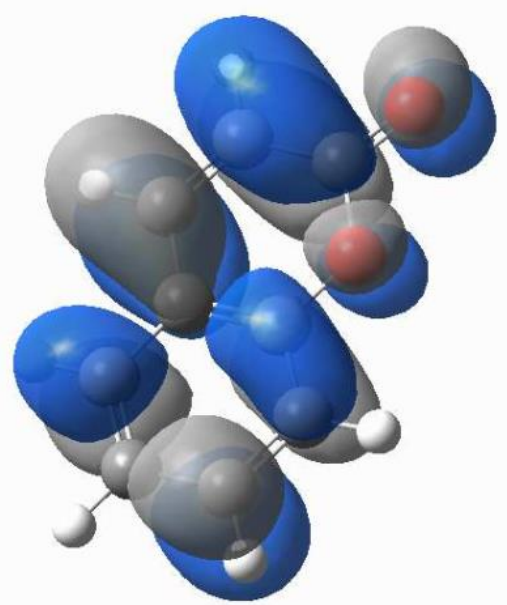

Coumarin - HOMO

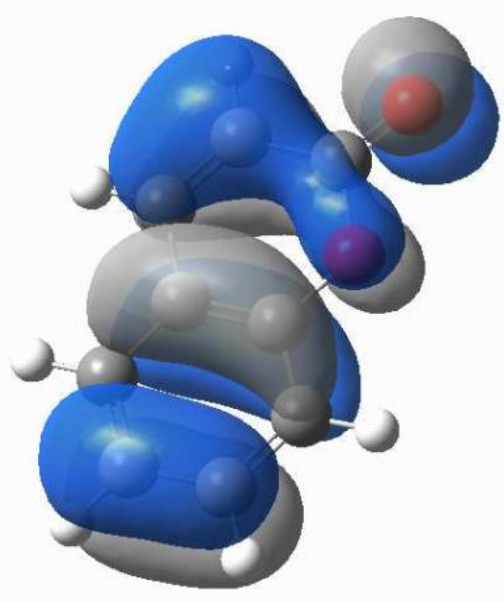

Coumarin - LUMO

\begin{tabular}{|c|c|c|c|c|c|}
\hline \multirow{2}{*}{$\begin{array}{l}\text { Center } \\
\text { Number }\end{array}$} & \multicolumn{2}{|l|}{ Atomic } & \multirow{2}{*}{$\begin{array}{l}\text { Atomic } \\
\text { Type }\end{array}$} & \multicolumn{2}{|c|}{ Coordinates (Angstron } \\
\hline & Numbe & & & $\begin{array}{ll}X & Y\end{array}$ & $\mathrm{Z}$ \\
\hline 1 & 6 & 0 & -1.294975 & -1.486715 & 0.000046 \\
\hline 2 & 6 & 0 & -2.598844 & -1.006884 & 0.000132 \\
\hline 3 & 6 & 0 & -2.856971 & 0.371374 & 0.000131 \\
\hline 4 & 6 & 0 & -1.803 & 1.271625 & 0.000043 \\
\hline 5 & 6 & 0 & -0.473 & 0.812669 & -0.000100 \\
\hline 6 & 6 & 0 & -0.2436 & -0.572968 & -0.000096 \\
\hline 7 & 6 & 0 & 0.675493 & 1.677816 & 0.000048 \\
\hline 8 & 6 & 0 & 1.926 & 1.166563 & -0.000078 \\
\hline 9 & 6 & 0 & 2.1568 & -0.267043 & -0.001173 \\
\hline 10 & 8 & 0 & 1.030305 & -1.075024 & -0.000322 \\
\hline 11 & 8 & & 3.235129 & -0.819019 & 0.000912 \\
\hline 12 & 1 & & -1.079306 & -2.548269 & 0.000039 \\
\hline 13 & 1 & & -3.423707 & -1.710301 & 0.000221 \\
\hline 14 & 1 & 0 & -3.879310 & 0.730257 & 0.000237 \\
\hline 15 & 1 & 0 & -1.988773 & 2.340357 & 0.000115 \\
\hline 16 & 1 & 0 & 0.519709 & 2.751702 & 0.000493 \\
\hline 17 & 1 & 0 & 2.810663 & 1.789974 & 0.000463 \\
\hline
\end{tabular}




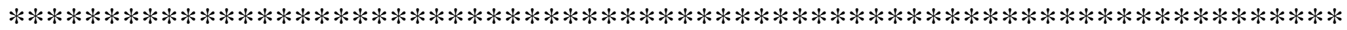

Population analysis using the SCF density.

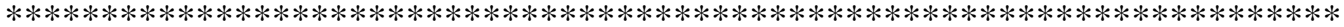

Orbital symmetries:

Occupied (A) (A) (A) (A) (A) (A) (A) (A) (A) (A) (A) (A)

(A) $(\mathrm{A})(\mathrm{A})(\mathrm{A})(\mathrm{A})(\mathrm{A})(\mathrm{A})(\mathrm{A})(\mathrm{A})(\mathrm{A})(\mathrm{A})(\mathrm{A})$

$(\mathrm{A})(\mathrm{A})(\mathrm{A})(\mathrm{A})(\mathrm{A})(\mathrm{A})(\mathrm{A})(\mathrm{A})(\mathrm{A})(\mathrm{A})(\mathrm{A})(\mathrm{A})$

$(\mathrm{A})(\mathrm{A})$

Virtual (A) (A) (A) (A) (A) (A) (A) (A) (A) (A) (A) (A)

(A) $(\mathrm{A})(\mathrm{A})(\mathrm{A})(\mathrm{A})(\mathrm{A})(\mathrm{A})(\mathrm{A})(\mathrm{A})(\mathrm{A})(\mathrm{A})(\mathrm{A})$

(A) $(\mathrm{A})(\mathrm{A})(\mathrm{A})(\mathrm{A})(\mathrm{A})(\mathrm{A})(\mathrm{A})(\mathrm{A})(\mathrm{A})(\mathrm{A})(\mathrm{A})$

(A) $(\mathrm{A})(\mathrm{A})(\mathrm{A})(\mathrm{A})(\mathrm{A})(\mathrm{A})(\mathrm{A})(\mathrm{A})(\mathrm{A})(\mathrm{A})(\mathrm{A})$

(A) $(\mathrm{A})(\mathrm{A})(\mathrm{A})(\mathrm{A})(\mathrm{A})(\mathrm{A})(\mathrm{A})(\mathrm{A})(\mathrm{A})(\mathrm{A})(\mathrm{A})$

(A) $(\mathrm{A})(\mathrm{A})(\mathrm{A})(\mathrm{A})(\mathrm{A})(\mathrm{A})(\mathrm{A})(\mathrm{A})(\mathrm{A})(\mathrm{A})(\mathrm{A})$

(A)

The electronic state is 1-A.

Alpha occ. eigenvalues -- -19.21355 -19.13292 -10.32651 -10.27708 -10.23230

Alpha occ. eigenvalues -- $-10.22340-10.21596-10.21465-10.21272-10.20756$

Alpha occ. eigenvalues -- -10.20652 -1.13495 -1.04841 -0.89042 -0.84250

Alpha occ. eigenvalues -- $-0.77938-0.77020-0.67176-0.66454-0.64021$

Alpha occ. eigenvalues -- $-0.57576 \quad-0.54560 \quad-0.51122-0.48812-0.48119$

Alpha occ. eigenvalues -- $-0.46708 \quad-0.46028 \quad-0.43501 \quad-0.42791 \quad-0.39963$

Alpha occ. eigenvalues -- $-0.39486-0.38462 \quad-0.35657 \quad-0.34934-0.32576$

Alpha occ. eigenvalues -- $-0.27482-0.26434-0.24344$

$\begin{array}{llllll}\text { Alpha virt. eigenvalues -- } & -0.07287 & -0.01691 & -0.00183 & 0.08055 & 0.08083\end{array}$

$\begin{array}{lllllll}\text { Alpha virt. eigenvalues -- } & 0.09235 & 0.13245 & 0.14404 & 0.15889 & 0.16583\end{array}$

$\begin{array}{llllllll}\text { Alpha virt. eigenvalues -- } & 0.16606 & 0.17830 & 0.21927 & 0.26768 & 0.27592\end{array}$

$\begin{array}{lllllll}\text { Alpha virt. eigenvalues -- } & 0.29076 & 0.31224 & 0.31800 & 0.33101 & 0.43327\end{array}$

$\begin{array}{lllllll}\text { Alpha virt. eigenvalues -- } & 0.45429 & 0.47347 & 0.47568 & 0.50528 & 0.51981\end{array}$

$\begin{array}{lllllll}\text { Alpha virt. eigenvalues -- } & 0.54192 & 0.55843 & 0.56165 & 0.58332 & 0.58391\end{array}$

$\begin{array}{lllllll}\text { Alpha virt. eigenvalues -- } & 0.59982 & 0.61095 & 0.61450 & 0.62406 & 0.63279\end{array}$

$\begin{array}{lllllll}\text { Alpha virt. eigenvalues -- } & 0.63893 & 0.65526 & 0.65544 & 0.66727 & 0.69503\end{array}$

$\begin{array}{llllllll}\text { Alpha virt. eigenvalues -- } & 0.70290 & 0.77604 & 0.81105 & 0.81803 & 0.83912\end{array}$

$\begin{array}{lllllll}\text { Alpha virt. eigenvalues -- } & 0.85172 & 0.87269 & 0.88262 & 0.91586 & 0.93106\end{array}$

$\begin{array}{lllllll}\text { Alpha virt. eigenvalues -- } & 0.95324 & 0.96780 & 1.00790 & 1.01154 & 1.02263\end{array}$

$\begin{array}{lllllll}\text { Alpha virt. eigenvalues -- } & 1.05048 & 1.06601 & 1.08446 & 1.09497 & 1.13436\end{array}$

$\begin{array}{llllll}\text { Alpha virt. eigenvalues -- } & 1.16323 & 1.17862 & 1.23606 & 1.25370 & 1.30614\end{array}$

$\begin{array}{lllllll}\text { Alpha virt. eigenvalues -- } & 1.40172 & 1.43019 & 1.46151 & 1.59395 & 1.78850\end{array}$

Alpha virt. eigenvalues -- $1.87142 \quad 1.94246 \quad 2.16583$

The Molecular Orbital Coefficients for MOs 34 through 43 are shown here ( 38 is the HOMO and 39 the LUMO):

$\begin{array}{rrrrr}34 & 35 & 36 & 37 & 38 \\ \mathrm{O} & \mathrm{O} & \mathrm{O} & \mathrm{O} & \mathrm{O}\end{array}$

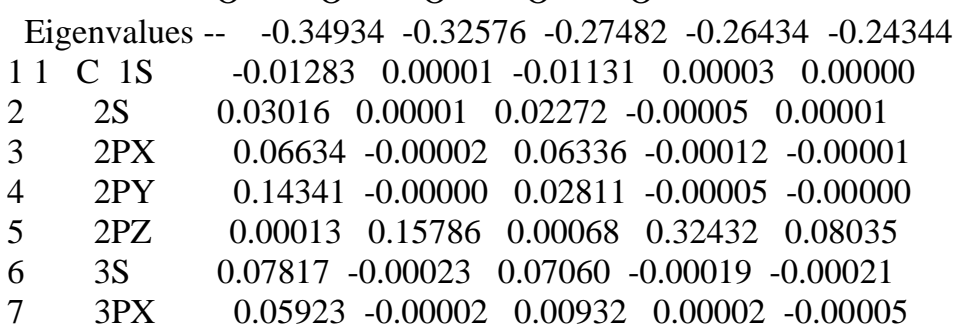




\begin{tabular}{|c|c|c|}
\hline 8 & 3PY & $\begin{array}{lllll}0.03012 & -0.00016 & 0.03175 & -0.00009 & -0.00016\end{array}$ \\
\hline 9 & 3PZ & $\begin{array}{lllll}0.00008 & 0.10905 & 0.00047 & 0.23400 & 0.06893\end{array}$ \\
\hline 102 & C $1 S$ & $\begin{array}{lllll}0.00431 & -0.00000 & 0.00530 & -0.00001 & -0.00000\end{array}$ \\
\hline & $2 \mathrm{~S}$ & $\begin{array}{lllll}0.00772 & 0.00001 & -0.01171 & 0.00003 & 0.00001\end{array}$ \\
\hline 12 & $2 \mathrm{PX}$ & $\begin{array}{lllll}-0.01392 & 0.00005 & -0.03877 & 0.00010 & 0.00000\end{array}$ \\
\hline & $2 \mathrm{PY}$ & $0.00000-0.00001$ \\
\hline & $2 \mathrm{PZ}$ & $\begin{array}{lll}-0.00007 & 0.26753 & 0.00049\end{array}$ \\
\hline 5 & $3 \mathrm{~S}$ & $\begin{array}{lllll}-0.00026 & 0.00011 & -0.04416 & 0.00014 & 0.00008\end{array}$ \\
\hline 6 & $3 \mathrm{PX}$ & $\begin{array}{lllll}-0.00320 & 0.00006 & -0.03149 & 0.00010 & 0.00002\end{array}$ \\
\hline 17 & 3PY & $\begin{array}{lllll}-0.05476 & 0.00005 & 0.00552 & -0.00001 & 0.00004\end{array}$ \\
\hline 18 & 3PZ & $\begin{array}{lllll}-0.00005 & 0.16969 & 0.00035 & 0.13064 & -0.13661\end{array}$ \\
\hline 193 & C $1 S$ & $\begin{array}{lllll}-0.00087 & -0.00000 & 0.00062 & -0.00000 & -0.00000\end{array}$ \\
\hline 20 & $2 \mathrm{~S}$ & $\begin{array}{lllll}0.00327 & 0.00001 & -0.00212 & 0.00000 & 0.00000\end{array}$ \\
\hline 21 & $2 \mathrm{PX}$ & $\begin{array}{lllll}0.01659 & 0.00004 & 0.00740 & -0.00004 & -0.00002\end{array}$ \\
\hline 22 & $2 \mathrm{PY}$ & $\begin{array}{llll}0.00001 & 0.00558 & -0.00002 & -0.00000\end{array}$ \\
\hline 3 & $2 \mathrm{PZ}$ & $\begin{array}{llll}0.22777 & -0.00021 & -0.16979 & -0.2\end{array}$ \\
\hline 24 & $3 \mathrm{~S}$ & $\begin{array}{lllll}-0.01256 & -0.00005 & 0.01227 & -0.00003 & -0.00003\end{array}$ \\
\hline & 3PX & $\begin{array}{lllll}-0.00739 & -0.00001 & 0.01525 & -0.00005 & -0.00002\end{array}$ \\
\hline 6 & 3PY & $\begin{array}{lllll}0.03835 & 0.00004 & 0.00275 & -0.00000 & 0.0\end{array}$ \\
\hline 27 & $3 \mathrm{PZ}$ & $\begin{array}{lllll}-0.00015 & 0.14938 & -0.00015 & -0.12336 & -0.17156\end{array}$ \\
\hline & C $1 S$ & $\begin{array}{lllll}-0.01042 & 0.00000 & -0.00225 & 0.00000 & -0\end{array}$ \\
\hline 29 & $2 \mathrm{~S}$ & $\begin{array}{lllll}0.02564 & -0.00001 & 0.00578 & -0.00001 & 0.00\end{array}$ \\
\hline 30 & $2 \mathrm{PX}$ & $\begin{array}{lllll}0.03238 & -0.00001 & -0.00751 & -0.00001 & 0 .\end{array}$ \\
\hline 31 & $2 \mathrm{PY}$ & $\begin{array}{llll}-0.14082 & -0.00001 & -0.00276 & 0.00002\end{array}$ \\
\hline 32 & $2 \mathrm{PZ}$ & $\begin{array}{lllll}-0.00036 & 0.06587 & -0.00068 & -0.32368 & 0 .\end{array}$ \\
\hline & $3 \mathrm{~S}$ & $\begin{array}{lllll}0.05646 & -0.00002 & -0.02432 & 0.00006 & -0.0\end{array}$ \\
\hline 34 & 3PX & $\begin{array}{lllll}0.00092 & -0.00000 & -0.00815 & 0.00001 & -0 .\end{array}$ \\
\hline 5 & 3PY & $\begin{array}{lllll}-0.07337 & -0.00003 & 0.02593 & -0.00005 & 0 .\end{array}$ \\
\hline 36 & $3 \mathrm{PZ}$ & $\begin{array}{lllll}-0.00023 & 0.04365 & -0.00049 & -0.23879 & 0 .\end{array}$ \\
\hline 375 & C $1 S$ & $\begin{array}{lllll}0.00616 & -0.00001 & -0.00051 & 0.00000 & -0\end{array}$ \\
\hline & $2 \mathrm{~S}$ & $\begin{array}{lllll}-0.00757 & 0.00003 & 0.00166 & -0.00001 & 0.0\end{array}$ \\
\hline 39 & $2 \mathrm{PX}$ & $\begin{array}{lllll}-0.01734 & 0.00003 & 0.01906 & -0.00006 & 0 .\end{array}$ \\
\hline & $2 \mathrm{PY}$ & $\begin{array}{lllll}0.25079 & 0.00002 & 0.04006 & -0.00011 & 0.0\end{array}$ \\
\hline & $2 \mathrm{PZ}$ & $\begin{array}{lllll}-0.00031 & -0.15609 & -0.00046 & -0.11728 & 0 .\end{array}$ \\
\hline & $3 \mathrm{~S}$ & 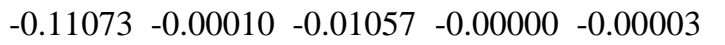 \\
\hline & 3PX & $\begin{array}{lllll}0.01864 & 0.00000 & -0.05434 & 0.00012 & -0 .\end{array}$ \\
\hline 44 & 3PY & $\begin{array}{lllll}0.18229 & 0.00019 & -0.01351 & 0.00005 & 0 .\end{array}$ \\
\hline & $3 \mathrm{PZ}$ & $\begin{array}{lllll}-0.00021 & -0.09331 & -0.00029 & -0.07552 & 0 .\end{array}$ \\
\hline 466 & C $1 S$ & $\begin{array}{lllll}-0.00967 & -0.00001 & 0.01050 & -0.00002 & -0\end{array}$ \\
\hline 47 & $2 \mathrm{~S}$ & $\begin{array}{lllll}0.02655 & 0.00003 & -0.02230 & 0.00004 & 0.00004\end{array}$ \\
\hline 48 & $2 \mathrm{PX}$ & $\begin{array}{lllll}0.04850 & 0.00009 & -0.08107 & 0.00019 & 0.0\end{array}$ \\
\hline 49 & $2 \mathrm{PY}$ & $\begin{array}{lllll}-0.25603 & -0.00001 & -0.02138 & 0.00005 & -0.00001\end{array}$ \\
\hline 50 & $2 \mathrm{PZ}$ & $\begin{array}{lllll}0.00033 & -0.05292 & 0.00021 & 0.11940 & 0.2\end{array}$ \\
\hline 51 & $3 \mathrm{~S}$ & $\begin{array}{lllll}0.04798 & 0.00030 & -0.05148 & 0.00012 & 0.00027\end{array}$ \\
\hline & 3PX & $\begin{array}{lllll}-0.03672 & -0.00021 & 0.00894 & -0.00004 & -0.00019\end{array}$ \\
\hline 53 & 3PY & $\begin{array}{lllll}0.05233 & 0.00003 & -0.02790 & 0.00008 & -0.00002\end{array}$ \\
\hline 54 & $3 \mathrm{PZ}$ & $\begin{array}{lllll}0.00023 & -0.04311 & 0.00016 & 0.07126 & 0.15\end{array}$ \\
\hline & C $1 S$ & $\begin{array}{lllll}-0.00301 & -0.00001 & -0.01730 & 0.00004 & -0.00002\end{array}$ \\
\hline 56 & $2 \mathrm{~S}$ & $\begin{array}{lllll}0.01338 & 0.00000 & 0.02875 & -0.00007 & 0.00002\end{array}$ \\
\hline & $2 \mathrm{PX}$ & $\begin{array}{lllll}-0.07445 & 0.00001 & 0.04674 & -0.00009 & 0.00008\end{array}$ \\
\hline 58 & $2 \mathrm{PY}$ & $\begin{array}{lllll}-0.11139 & 0.00006 & -0.06835 & 0.00013 & -0.00005\end{array}$ \\
\hline & $2 \mathrm{PZ}$ & $\begin{array}{lllll}-0.00051 & -0.25180 & 0.00006 & 0.12160 & -0.10219\end{array}$ \\
\hline 60 & $3 \mathrm{~S}$ & $\begin{array}{lllll}-0.02490 & 0.00044 & 0.17496 & -0.00027 & 0.00064\end{array}$ \\
\hline 61 & 3PX & $\begin{array}{lllll}-0.01084 & 0.00024 & 0.03268 & 0.00001 & 0.00037\end{array}$ \\
\hline 62 & 3PY & $-0.04043-0.00015-0.11251$ \\
\hline 63 & $3 \mathrm{PZ}$ & $\begin{array}{lll}-0.00030 & -0.17579 & -0.00009\end{array}$ \\
\hline
\end{tabular}




\begin{tabular}{|c|c|c|}
\hline 648 & C $1 S$ & $\begin{array}{lllll}-0.00010 & 0.00001 & 0.03283 & -0.00008 & 0.00005\end{array}$ \\
\hline 65 & $2 \mathrm{~S}$ & $\begin{array}{llllll}0.00012 & 0.00001 & -0.05305 & 0.00014 & -0.00008\end{array}$ \\
\hline 66 & $2 \mathrm{PX}$ & $\begin{array}{lllll}0.04735 & 0.00003 & -0.05650 & 0.00008 & -0.00005\end{array}$ \\
\hline 67 & $2 \mathrm{PY}$ & $\begin{array}{llll}0.00021 & 0.17880 & -0.00055 & 0.00045\end{array}$ \\
\hline 68 & $2 \mathrm{PZ}$ & $\begin{array}{lllll}-0.00030 & -0.16023 & 0.00050 & 0.17703 & -0.24038\end{array}$ \\
\hline 99 & $3 \mathrm{~S}$ & $\begin{array}{lllll}-0.02326 & -0.00060 & -0.38734 & 0.00085 & -0.00093\end{array}$ \\
\hline 0 & 3PX & $\begin{array}{lllll}0.01478 & 0.00059 & 0.01107 & 0.00010 & 0.00071\end{array}$ \\
\hline 71 & 3PY & $0.17844-0.00057 \quad 0.00044$ \\
\hline 72 & 3PZ & $0.00062 \quad 0.14484-0.18901$ \\
\hline 739 & C $1 S$ & $\begin{array}{lllll}-0.00784 & 0.00003 & 0.01607 & -0.00004 & -0.00002\end{array}$ \\
\hline 74 & $2 \mathrm{~S}$ & $\begin{array}{llllll}0.02762 & -0.00011 & -0.04748 & 0.00013 & -0.00002\end{array}$ \\
\hline 75 & $2 \mathrm{PX}$ & $\begin{array}{lllll}-0.04789 & 0.00001 & 0.04390 & -0.00012 & 0.00002\end{array}$ \\
\hline 6 & $2 \mathrm{PY}$ & $\begin{array}{lllll}-0.17221 & -0.00009 & -0.04893 & 0.00012 & -0.00013\end{array}$ \\
\hline 77 & $2 \mathrm{PZ}$ & $\begin{array}{lllll}-0.00029 & 0.11220 & 0.00008 & 0.00029 & -0.02676\end{array}$ \\
\hline 8 & $3 \mathrm{~S}$ & $\begin{array}{lllll}-0.01859 & 0.00001 & -0.02143 & -0.00000 & 0.00013\end{array}$ \\
\hline 9 & 3PX & $\begin{array}{lllll}-0.07304 & -0.00072 & 0.10285 & -0.00033 & -0.00072\end{array}$ \\
\hline 80 & 3PY & $\begin{array}{lllll}-0.00791 & 0.00020 & 0.24900 & -0.00065 & 0.00032\end{array}$ \\
\hline 81 & $3 \mathrm{PZ}$ & $\begin{array}{lllll}-0.00024 & 0.06588 & -0.00002 & -0.01577 & -0.00946\end{array}$ \\
\hline 821 & $\mathrm{O} 1 \mathrm{~S}$ & $\begin{array}{lllll}0.04508 & 0.00002 & -0.02553 & 0.00006 & 0.00002\end{array}$ \\
\hline 83 & $2 \mathrm{~S}$ & $\begin{array}{lllll}-0.10486 & -0.00001 & 0.04379 & -0.00011 & -0.00001\end{array}$ \\
\hline 84 & $2 \mathrm{PX}$ & $\begin{array}{lllll}-0.07564 & -0.00012 & 0.14833 & -0.00040 & -0.00010\end{array}$ \\
\hline 85 & $2 \mathrm{PY}$ & $\begin{array}{lllll}0.37442 & -0.00001 & 0.15368 & -0.00043 & 0.00006\end{array}$ \\
\hline 86 & $2 \mathrm{PZ}$ & $\begin{array}{lllll}0.00078 & -0.07937 & -0.00021 & -0.17892 & -0.22213\end{array}$ \\
\hline 37 & $3 \mathrm{~S}$ & $\begin{array}{lllll}-0.15217 & -0.00030 & 0.24002 & -0.00059 & -0.00021\end{array}$ \\
\hline 88 & 3PX & $\begin{array}{lllll}-0.06650 & -0.00013 & 0.16765 & -0.00047 & -0.00009\end{array}$ \\
\hline 89 & $3 P Y$ & $\begin{array}{lllll}0.24151 & -0.00016 & 0.15533 & -0.00041 & -0.00008\end{array}$ \\
\hline 90 & $3 \mathrm{PZ}$ & $\begin{array}{lllll}0.00061 & -0.06460 & -0.00028 & -0.13928 & -0.18651\end{array}$ \\
\hline 9111 & $\mathrm{O} 1 \mathrm{~S}$ & $\begin{array}{lllll}0.00395 & 0.00000 & -0.00009 & 0.00000 & -0.00002\end{array}$ \\
\hline 92 & $2 \mathrm{~S}$ & 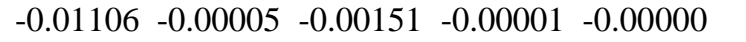 \\
\hline 93 & $2 \mathrm{PX}$ & $\begin{array}{lllll}-0.14235 & -0.00074 & 0.24289 & -0.00044 & -0.00049\end{array}$ \\
\hline 94 & $2 \mathrm{PY}$ & $\begin{array}{lllll}-0.09729 & 0.00017 & 0.54629 & -0.00111 & 0.00060\end{array}$ \\
\hline 95 & $2 \mathrm{PZ}$ & $\begin{array}{llll}-0.00079 & 0.25659 & -0.00035 & -0.01190\end{array}$ \\
\hline 96 & $3 \mathrm{~S}$ & $\begin{array}{lllll}0.02711 & 0.00036 & 0.02290 & 0.00001 & 0.00056\end{array}$ \\
\hline 97 & 3PX & $\begin{array}{lllll}-0.09704 & -0.00056 & 0.15122 & -0.00027 & -0.00047\end{array}$ \\
\hline 98 & 3PY & $\begin{array}{lllll}-0.05166 & 0.00017 & 0.34771 & -0.00066 & 0.00051\end{array}$ \\
\hline 99 & $3 \mathrm{PZ}$ & $\begin{array}{lllll}-0.00056 & 0.17539 & -0.00026 & -0.00589 & 0.22578\end{array}$ \\
\hline 100 & $2 \mathrm{H} 1 \mathrm{~S}$ & $\begin{array}{lllll}-0.09617 & -0.00001 & 0.00314 & -0.00001 & -0.00001\end{array}$ \\
\hline 101 & $2 \mathrm{~S}$ & $\begin{array}{lllll}-0.13185 & -0.00006 & -0.00081 & -0.00001 & -0.00007\end{array}$ \\
\hline & $3 \mathrm{H} 1 \mathrm{~S}$ & $\begin{array}{lllll}0.06881 & -0.00001 & 0.01918 & -0.00005 & -0.00001\end{array}$ \\
\hline 103 & $2 \mathrm{~S}$ & $\begin{array}{lllll}0.06527 & 0.00000 & 0.02419 & -0.00006 & -0.00000\end{array}$ \\
\hline 10414 & $4 \mathrm{H} 1 \mathrm{~S}$ & $\begin{array}{llllll}0.02511 & -0.00000 & -0.00705 & 0.00001 & 0.00000\end{array}$ \\
\hline 105 & $2 \mathrm{~S}$ & $\begin{array}{llllll}0.02112 & -0.00002 & -0.00473 & 0.00001 & -0.00000\end{array}$ \\
\hline & $5 \mathrm{H} 1 \mathrm{~S}$ & $\begin{array}{lllll}-0.11004 & -0.00001 & -0.00302 & 0.00001 & 0.00000\end{array}$ \\
\hline 107 & $2 \mathrm{~S}$ & $\begin{array}{lllll}-0.11740 & 0.00001 & -0.01902 & 0.00006 & -0.00001\end{array}$ \\
\hline & $6 \mathrm{H} 1 \mathrm{~S}$ & $\begin{array}{lllll}-0.08618 & -0.00003 & -0.05148 & 0.00015 & -0.00010\end{array}$ \\
\hline & $2 \mathrm{~S}$ & $\begin{array}{lllll}-0.09425 & 0.00002 & -0.04596 & 0.00014 & -0.00007\end{array}$ \\
\hline & $7 \mathrm{H} 1 \mathrm{~S}$ & $\begin{array}{lllll}0.09360 & 0.00003 & 0.01286 & -0.00006 & 0.00001\end{array}$ \\
\hline \multirow[t]{3}{*}{111} & $2 \mathrm{~S}$ & $\begin{array}{llllll}0.08129 & -0.00018 & 0.03769 & -0.00015 & -0.00020\end{array}$ \\
\hline & & 42 \\
\hline & & $\mathrm{V}$ \\
\hline
\end{tabular}

$\begin{array}{llllll}\text { Eigenvalues -- } & -0.07287 & -0.01691 & -0.00183 & 0.08055 & 0.08083\end{array}$

$\begin{array}{llllllll}11 & \mathrm{C} & 1 \mathrm{~S} & -0.00000 & 0.00001 & -0.00001 & -0.00321 & 0.02390\end{array}$

$\begin{array}{llllllll}2 & 2 \mathrm{~S} & 0.00001 & -0.00003 & 0.00001 & 0.00476 & -0.03599\end{array}$

$\begin{array}{llllllll}3 & 2 \mathrm{PX} & -0.00000 & -0.00007 & 0.00001 & -0.00189 & 0.01317\end{array}$

$\begin{array}{llllllll}4 & 2 \mathrm{PY} & 0.00000 & -0.00004 & 0.00003 & -0.01415 & 0.10160\end{array}$

$\begin{array}{lllllll}5 & 2 \mathrm{PZ} & -0.12169 & -0.24044 & -0.24346 & -0.17735 & -0.02411\end{array}$ 


\begin{tabular}{|c|c|c|}
\hline 6 & $3 \mathrm{~S}$ & $\begin{array}{lllll}0.00008 & -0.00008 & 0.00008 & 0.04801 & -0.35338\end{array}$ \\
\hline 7 & 3PX & $\begin{array}{lllll}-0.00012 & 0.00001 & 0.00002 & 0.01549 & -0.10945\end{array}$ \\
\hline 8 & 3PY & $\begin{array}{lllll}0.00005 & -0.00005 & 0.00007 & -0.02427 & 0.17551\end{array}$ \\
\hline 9 & 3PZ & $\begin{array}{lllll}-0.14283 & -0.32255 & -0.34533 & -0.27126 & -0.03687\end{array}$ \\
\hline 102 & C $1 S$ & $\begin{array}{lllll}-0.00000 & -0.00002 & 0.00002 & -0.00629 & 0.04549\end{array}$ \\
\hline 11 & $2 \mathrm{~S}$ & $\begin{array}{llllll}0.00001 & 0.00004 & -0.00005 & 0.01089 & -0.07853\end{array}$ \\
\hline 12 & $2 \mathrm{PX}$ & $\begin{array}{lllll}0.00002 & 0.00003 & -0.00003 & -0.01453 & 0.10905\end{array}$ \\
\hline 13 & $2 \mathrm{PY}$ & $\begin{array}{lllll}0.00001 & -0.00002 & 0.00004 & -0.01233 & 0.08870\end{array}$ \\
\hline 14 & $2 \mathrm{PZ}$ & $\begin{array}{lllll}0.22620 & -0.10216 & 0.25747 & 0.23609 & 0.03201\end{array}$ \\
\hline 15 & $3 \mathrm{~S}$ & $\begin{array}{lllll}-0.00009 & 0.00012 & -0.00015 & 0.08669 & -0.62823\end{array}$ \\
\hline 16 & 3PX & $\begin{array}{lllll}-0.00003 & 0.00002 & 0.00001 & -0.03308 & 0.24572\end{array}$ \\
\hline 17 & 3PY & $\begin{array}{llll}0.00002 & -0.00010 & 0.00008 & -0.04102\end{array}$ \\
\hline 18 & 3PZ & $\begin{array}{llllll}0.25440 & -0.13373 & 0.36889 & 0.38744 & 0.05254\end{array}$ \\
\hline 193 & C $1 S$ & $\begin{array}{lllll}-0.00000 & -0.00000 & 0.00001 & -0.00542 & 0.03987\end{array}$ \\
\hline 20 & $2 \mathrm{~S}$ & $\begin{array}{llllll}0.00000 & 0.00000 & -0.00001 & 0.00800 & -0.05870\end{array}$ \\
\hline 21 & 2PX & $\begin{array}{lllll}0.00000 & 0.00003 & -0.00000 & -0.02129 & 0.15781\end{array}$ \\
\hline 22 & $2 \mathrm{PY}$ & $\begin{array}{lllll}-0.00000 & -0.00000 & -0.00001 & 0.00621 & -0.04622\end{array}$ \\
\hline 23 & $2 \mathrm{PZ}$ & $\begin{array}{lllll}0.00953 & 0.34396 & -0.06208 & -0.22835 & -0.03099\end{array}$ \\
\hline 24 & $3 \mathrm{~S}$ & $\begin{array}{lllll}0.00005 & 0.00008 & -0.00013 & 0.09674 & -0.71132\end{array}$ \\
\hline 25 & 3PX & $\begin{array}{lllll}0.00005 & 0.00008 & -0.00005 & -0.04095 & 0.30382\end{array}$ \\
\hline 26 & 3PY & $\begin{array}{lllll}-0.00002 & -0.00003 & 0.00002 & 0.01297 & -0.09606\end{array}$ \\
\hline 27 & $3 \mathrm{PZ}$ & $\begin{array}{lllll}-0.00612 & 0.47253 & -0.06673 & -0.36446 & -0.04950\end{array}$ \\
\hline 284 & C $1 S$ & $\begin{array}{lllll}-0.00000 & -0.00001 & 0.00001 & -0.00625 & 0.04583\end{array}$ \\
\hline 29 & $2 \mathrm{~S}$ & $\begin{array}{lllll}0.00002 & 0.00002 & -0.00003 & 0.01091 & -0.07948\end{array}$ \\
\hline 30 & $2 \mathrm{PX}$ & $\begin{array}{lllll}-0.00002 & -0.00001 & -0.00003 & -0.00508 & 0.04032\end{array}$ \\
\hline 31 & $2 \mathrm{PY}$ & $\begin{array}{llllll}0.00003 & 0.00001 & -0.00002 & 0.02201 & -0.16463\end{array}$ \\
\hline 32 & $2 \mathrm{PZ}$ & $\begin{array}{lllll}-0.21006 & -0.24888 & -0.19472 & 0.14107 & 0.01917\end{array}$ \\
\hline 33 & $3 \mathrm{~S}$ & $\begin{array}{lllll}-0.00017 & -0.00006 & 0.00014 & 0.08854 & -0.65502\end{array}$ \\
\hline 34 & 3PX & $\begin{array}{lllll}-0.00015 & -0.00009 & 0.00011 & -0.00475 & 0.03994\end{array}$ \\
\hline 35 & 3PY & $\begin{array}{lllll}0.00017 & 0.00007 & -0.00020 & 0.06612 & -0.49314\end{array}$ \\
\hline 36 & $3 \mathrm{PZ}$ & $\begin{array}{lllll}-0.21740 & -0.34888 & -0.30334 & 0.20001 & 0.02723\end{array}$ \\
\hline 375 & C $1 S$ & $\begin{array}{lllll}-0.00002 & -0.00001 & 0.00003 & -0.00243 & 0.01771\end{array}$ \\
\hline 38 & $2 \mathrm{~S}$ & $\begin{array}{llllll}0.00004 & 0.00000 & -0.00004 & 0.00251 & -0.01889\end{array}$ \\
\hline 39 & $2 \mathrm{PX}$ & $\begin{array}{lllll}0.00008 & 0.00001 & -0.00006 & 0.00208 & -0.01393\end{array}$ \\
\hline 40 & 2PY & $\begin{array}{llllll}0.00006 & 0.00001 & -0.00007 & 0.00499 & -0.03693\end{array}$ \\
\hline 41 & $2 \mathrm{PZ}$ & $\begin{array}{lllll}0.11093 & -0.09303 & 0.31907 & 0.03680 & 0.00483\end{array}$ \\
\hline 42 & $3 \mathrm{~S}$ & $\begin{array}{lllll}0.00018 & 0.00020 & -0.00037 & 0.05058 & -0.36626\end{array}$ \\
\hline 43 & 3PX & $\begin{array}{lllll}-0.00029 & -0.00004 & 0.00030 & 0.01915 & -0.13662\end{array}$ \\
\hline 44 & 3PY & $\begin{array}{lllll}-0.00004 & 0.00016 & -0.00011 & -0.00416 & 0.03372\end{array}$ \\
\hline 45 & $3 \mathrm{PZ}$ & $\begin{array}{lllll}0.10425 & -0.10333 & 0.45151 & 0.03530 & 0.00452\end{array}$ \\
\hline 466 & C $1 S$ & $\begin{array}{lllll}-0.00000 & -0.00003 & 0.00003 & -0.00508 & 0.03628\end{array}$ \\
\hline 47 & $2 \mathrm{~S}$ & $\begin{array}{llllll}0.00002 & 0.00005 & -0.00004 & 0.01081 & -0.07849\end{array}$ \\
\hline 48 & 2PX & $\begin{array}{llllll}0.00005 & 0.00020 & -0.00023 & 0.01190 & -0.07991\end{array}$ \\
\hline 49 & 2PY & $\begin{array}{lllll}-0.00006 & -0.00005 & 0.00013 & -0.00091 & 0.00210\end{array}$ \\
\hline 50 & $2 \mathrm{PZ}$ & $\begin{array}{lllll}-0.16061 & 0.38691 & 0.01339 & 0.04449 & 0.00619\end{array}$ \\
\hline 51 & $3 \mathrm{~S}$ & $\begin{array}{lllll}0.00014 & 0.00025 & -0.00046 & 0.02043 & -0.14046\end{array}$ \\
\hline 52 & 3PX & $\begin{array}{lllll}0.00024 & 0.00017 & -0.00036 & 0.02057 & -0.13800\end{array}$ \\
\hline 53 & 3PY & $\begin{array}{lllll}-0.00017 & 0.00001 & 0.00022 & -0.00573 & 0.03631\end{array}$ \\
\hline 54 & 3PZ & $\begin{array}{lllll}-0.15108 & 0.44235 & 0.01486 & 0.06194 & 0.00859\end{array}$ \\
\hline 557 & C $1 S$ & $\begin{array}{lllll}-0.00002 & 0.00000 & -0.00000 & -0.00026 & 0.00366\end{array}$ \\
\hline 56 & $2 \mathrm{~S}$ & $\begin{array}{llllll}0.00003 & -0.00002 & 0.00004 & 0.00010 & -0.00397\end{array}$ \\
\hline 57 & $2 \mathrm{PX}$ & $\begin{array}{lllll}0.00007 & 0.00006 & -0.00014 & 0.00652 & -0.04561\end{array}$ \\
\hline 58 & 2PY & $\begin{array}{lllll}-0.00014 & 0.00004 & 0.00004 & 0.02010 & -0.15134\end{array}$ \\
\hline 59 & $2 \mathrm{PZ}$ & $\begin{array}{lllll}0.32344 & 0.00099 & -0.22113 & -0.21417 & -0.02898\end{array}$ \\
\hline 60 & $3 \mathrm{~S}$ & $\begin{array}{lllll}0.00038 & 0.00022 & -0.00056 & 0.01453 & -0.13546\end{array}$ \\
\hline 61 & 3PX & $\begin{array}{lllll}0.00004 & 0.00030 & -0.00038 & 0.01904 & -0.13200\end{array}$ \\
\hline
\end{tabular}




\begin{tabular}{|c|c|c|}
\hline 62 & $3 P Y$ & $0.00000 \quad 0.00041$ \\
\hline 63 & $3 \mathrm{PZ}$ & $\begin{array}{lllll}0.37872 & -0.03355 & -0.31779 & -0.34806 & -0.04720\end{array}$ \\
\hline 8 & C $1 S$ & $\begin{array}{lllll}0.00008 & 0.00005 & -0.00012 & -0.00016 & 0.00413\end{array}$ \\
\hline & $2 \mathrm{~S}$ & $\begin{array}{lllll}-0.00017 & -0.00013 & 0.00025 & -0.00067 & -0.00181\end{array}$ \\
\hline & $2 \mathrm{PX}$ & $\begin{array}{lllll}-0.00004 & -0.00005 & 0.00012 & 0.00453 & -0.03922\end{array}$ \\
\hline & 2PY & $\begin{array}{lll}0.00054 & 0.00012 & -0.00045\end{array}$ \\
\hline & $2 \mathrm{PZ}$ & $\begin{array}{lll}-0.27552 & 0.08529 & -0.03478\end{array}$ \\
\hline & $3 \mathrm{~S}$ & $\begin{array}{lllll}-0.00105 & -0.00040 & 0.00165 & 0.01377 & -0.13492\end{array}$ \\
\hline & 3PX & $\begin{array}{lllll}0.00012 & 0.00026 & -0.00044 & 0.00726 & -0 .\end{array}$ \\
\hline & 3PY & $\begin{array}{lllll}0.00088 & -0.00002 & -0.00080 & 0.00281 & -0 .\end{array}$ \\
\hline & 3PZ & $\begin{array}{lllll}-0.34460 & 0.13949 & 0.00295 & 0.50479 & 0.0\end{array}$ \\
\hline & C $1 S$ & $\begin{array}{lllll}-0.00011 & -0.00012 & 0.00023 & -0.00338 & 0 .\end{array}$ \\
\hline & $2 \mathrm{~S}$ & $\begin{array}{lllll}0.00010 & 0.00019 & -0.00024 & 0.00578 & -0.03\end{array}$ \\
\hline & $2 \mathrm{PX}$ & $\begin{array}{lllll}0.00009 & -0.00008 & 0.00003 & -0.00458 & 0 .\end{array}$ \\
\hline & 2PY & $\begin{array}{lllll}-0.00003 & -0.00017 & 0.00018 & -0.00034 & -0 .\end{array}$ \\
\hline & $2 \mathrm{PZ}$ & $\begin{array}{lllll}-0.19883 & -0.15535 & 0.29897 & -0.41888 & -0 .\end{array}$ \\
\hline & $3 \mathrm{~S}$ & $\begin{array}{lllll}0.00119 & 0.00105 & -0.00252 & 0.03218 & -0.1\end{array}$ \\
\hline & $3 \mathrm{PX}$ & $\begin{array}{lllll}0.00001 & -0.00025 & 0.00055 & 0.00201 & -0 .\end{array}$ \\
\hline & 3PY & $\begin{array}{lllll}0.00061 & -0.00031 & -0.00033 & -0.00805 & 0 .\end{array}$ \\
\hline & $3 \mathrm{PZ}$ & $\begin{array}{lllll}-0.13308 & -0.14753 & 0.29675 & -0.45728 & -0 .\end{array}$ \\
\hline & $\mathrm{O} 1 \mathrm{~S}$ & $\begin{array}{lllll}0.00002 & 0.00007 & -0.00011 & 0.00377 & -0\end{array}$ \\
\hline & $2 \mathrm{~S}$ & $\begin{array}{lllll}-0.00003 & -0.00015 & 0.00020 & -0.00777 & 0.0\end{array}$ \\
\hline & $2 \mathrm{PX}$ & $\begin{array}{lllll}0.00001 & -0.00016 & 0.00015 & 0.00992 & -0 .\end{array}$ \\
\hline & 2PY & $\begin{array}{lllll}0.00005 & -0.00016 & 0.00020 & -0.00996 & 0 .\end{array}$ \\
\hline 8 & $2 \mathrm{PZ}$ & $\begin{array}{lllll}0.18327 & -0.10929 & -0.12302 & 0.14398 & 0 .\end{array}$ \\
\hline 3 & 3 & $\begin{array}{lllll}-0.00021 & -0.00071 & 0.00118 & -0.03838 & 0.2\end{array}$ \\
\hline 38 & 3PX & $\begin{array}{lllll}0.00021 & -0.00025 & 0.00007 & 0.01214 & -0 .\end{array}$ \\
\hline 39 & 3PY & $\begin{array}{lllll}-0.00004 & -0.00024 & 0.00043 & -0.01185 & 0 .\end{array}$ \\
\hline 0 & 3PZ & $\begin{array}{lllll}0.17438 & -0.12168 & -0.13273 & 0.13469 & 0 .\end{array}$ \\
\hline & $\mathrm{O} 1 \mathrm{~S}$ & $\begin{array}{lllll}0.00000 & 0.00002 & -0.00003 & 0.00129 & -0\end{array}$ \\
\hline & $2 \mathrm{~S}$ & $\begin{array}{lllll}-0.00002 & -0.00006 & 0.00015 & -0.00280 & 0.0\end{array}$ \\
\hline & $2 \mathrm{PX}$ & $\begin{array}{llllll}-0.00020 & -0.00003 & 0.00003 & 0.00737 & -0 .\end{array}$ \\
\hline ). & $2 \mathrm{PY}$ & $\begin{array}{lllll}0.00025 & 0.00009 & -0.00019 & 0.00035 & 0 .\end{array}$ \\
\hline 95 & $2 \mathrm{PZ}$ & $\begin{array}{lllll}0.20980 & 0.11970 & -0.22365 & 0.22643 & 0 .\end{array}$ \\
\hline 96 & $3 \mathrm{~S}$ & $\begin{array}{lllll}0.00007 & -0.00010 & -0.00008 & -0.01530 & 0.0\end{array}$ \\
\hline 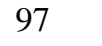 & $3 \mathrm{PX}$ & $\begin{array}{lllll}-0.00025 & 0.00001 & 0.00012 & 0.01008 & -0 .\end{array}$ \\
\hline 98 & 3PY & $\begin{array}{lllll}0.00010 & 0.00015 & -0.00019 & -0.00125 & 0.01213\end{array}$ \\
\hline 99 & 3PZ & $\begin{array}{lllll}0.19057 & 0.12250 & -0.23875 & 0.27124 & 0.0\end{array}$ \\
\hline & $2 \mathrm{H} 1 \mathrm{~S}$ & $\begin{array}{lllll}-0.00001 & -0.00003 & 0.00006 & -0.00847 & 0 .\end{array}$ \\
\hline & $2 \mathrm{~S}$ & $\begin{array}{lllll}-0.00002 & -0.00007 & 0.00017 & -0.07458 & 0.53\end{array}$ \\
\hline & $3 \mathrm{H} 1 \mathrm{~S}$ & $\begin{array}{lllll}-0.00000 & -0.00002 & 0.00003 & -0.01017 & 0.07399\end{array}$ \\
\hline & $2 \mathrm{~S}$ & $\begin{array}{lllll}-0.00002 & -0.00012 & 0.00017 & -0.12181 & 0.88929\end{array}$ \\
\hline & $+\mathrm{H} 1 \mathrm{~S}$ & $\begin{array}{lllll}-0.00001 & 0.00000 & 0.00000 & -0.00932 & 0.06924\end{array}$ \\
\hline 105 & $2 \mathrm{~S}$ & $\begin{array}{lllll}-0.00000 & -0.00000 & 0.00004 & -0.11866 & 0.87858\end{array}$ \\
\hline & H $1 \mathrm{~S}$ & $\begin{array}{lllll}-0.00001 & -0.00002 & 0.00002 & -0.00910 & 0.06660\end{array}$ \\
\hline 107 & $2 \mathrm{~S}$ & $\begin{array}{lllll}-0.00014 & -0.00008 & 0.00025 & -0.13498 & 1.00259\end{array}$ \\
\hline & $5 \mathrm{H} 1 \mathrm{~S}$ & $\begin{array}{lllll}-0.00008 & -0.00000 & 0.00003 & -0.00172 & 0.01407\end{array}$ \\
\hline & $2 \mathrm{~S}$ & $\begin{array}{lllll}-0.00017 & -0.00005 & 0.00001 & -0.07414 & 0.57459\end{array}$ \\
\hline & & $\begin{array}{lllll}-0.00002 & -0.00000 & 0.00001 & -0.00434 & 0.03450\end{array}$ \\
\hline & $2 \mathrm{~S}$ & $\begin{array}{lllll}-0.00007 & -0.00001 & 0.00004 & -0.02926 & 0.24773\end{array}$ \\
\hline
\end{tabular}




\section{X-ray diffraction details for $21 b$}

The crystal structure was solved using SHELXT 2014/5 (Sheldrick, 2014) and refined using SHELXL-2018/3 (Sheldrick, 2018). ${ }^{16}$ The space group P2 $1 /$ n was determined based on systematic absences and intensity statistics. A direct-methods solution was calculated, which provided most non-hydrogen atoms from the E-map. Full-matrix least-squares / difference Fourier cycles were performed that located the remaining non-hydrogen atoms. All of the nonhydrogen atoms were refined with anisotropic displacement parameters. All of the hydrogen atoms were placed in idealized positions and refined as riding atoms with relative isotropic displacement parameters. The structure of 21b was deposited in the Cambridge Crystallographic Data Centre (CCDC).

\section{Data for 21b (CCDC deposition number: 2057879)}

A red plate (approximate dimensions $0.200 \times 0.200 \times 0.020 \mathrm{~mm}^{3}$ ) was placed onto the tip of a 50 $\mu \mathrm{m}$ MiTeGen loop and mounted on a Bruker PHOTON-III CPAD diffractometer for a data collection at $125(2) \mathrm{K}$. A preliminary set of cell constants was calculated from reflections collected from three sets of frames. These initial sets were oriented such that orthogonal wedges of reciprocal space were surveyed. This produced an initial orientation matrix determined from 712 reflections. The data collection was carried out using MoK $\alpha$ radiation (parabolic mirrors) with a frame time of 20 seconds and a detector distance of $5.0 \mathrm{~cm}$. A strategy program was used to assure complete coverage of all unique data to a resolution of $0.70 \AA$. All major sections of frames were collected with $1.20^{\circ}$ steps in $\omega$ or $\phi$ at different detector positions in $2 \theta$. There are two molecules of chrysene in the asymmetric unit along with $1 / 2$ molecule of dichloromethane on an inversion. $\mathrm{C}_{62.50} \mathrm{H}_{55} \mathrm{Br}_{2} \mathrm{ClO}_{6} \mathrm{Si}_{2}$ refers to $2\left(\mathrm{C}_{31} \mathrm{H}_{27} \mathrm{BrO}_{3} \mathrm{Si}\right) \cdot 0.50\left(\mathrm{CH}_{2} \mathrm{Cl}_{2}\right)$

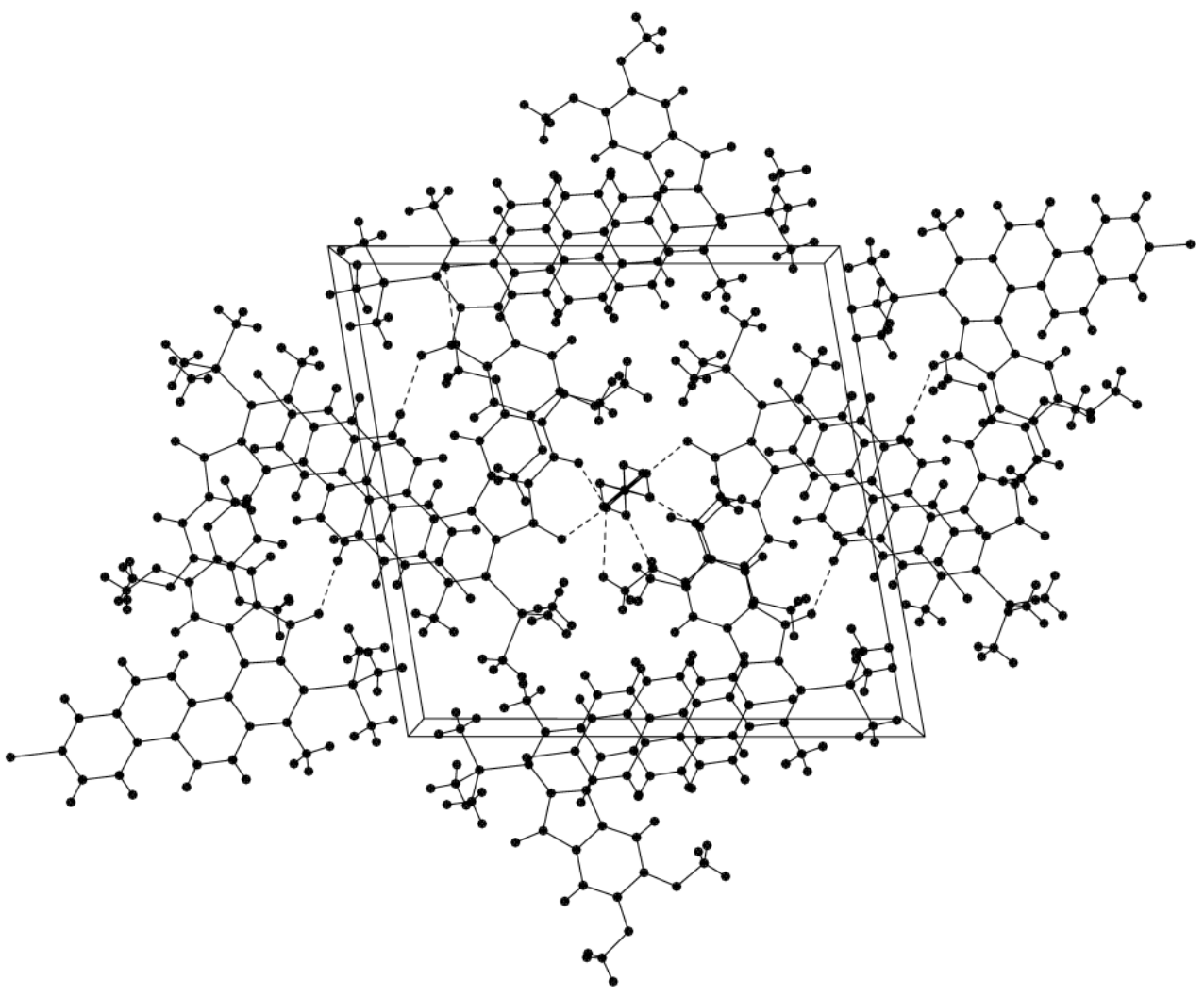




\section{Crystallographic details for $\mathbf{2 1 b}$}

Empirical formula

Formula weight

Temperature

Wavelength

Crystal system

Space group

Unit cell dimensions

Volume

$Z$

Density (calculated)

Absorption coefficient

$F(000)$

Crystal color, morphology

Crystal size

Theta range for data collection

Index ranges

Reflections collected

Independent reflections

Observed reflections

Completeness to theta $=25.242^{\circ}$

Absorption correction

Max. and min. transmission

Refinement method

Data / restraints / parameters

Goodness-of-fit on $F_{2}$

Final $R$ indices $[I>2 \operatorname{sigma}(I)]$

$R$ indices (all data)

Largest diff. peak and hole
$\mathrm{C}_{62.50} \mathrm{H}_{55} \mathrm{Br}_{2} \mathrm{Cl} \mathrm{O}_{6} \mathrm{Si}_{2}$

1153.51

125(2) K

$0.71073 \AA$

Triclinic

$\mathrm{P}-1$

$a=7.632(4) \AA$

$\mathrm{a}=80.459(15)^{\circ}$

$b=18.563(8) \AA$

$\mathrm{b}=89.746(19)^{\circ}$

$c=19.147(7) \AA$

$\mathrm{g}=87.12(3)^{\circ}$

2672(2) $\AA^{3}$

2

$1.434 \mathrm{Mg} / \mathrm{m}^{3}$

$1.665 \mathrm{~mm}^{-1}$

1186

Red, Plate

$0.200 \times 0.200 \times 0.020 \mathrm{~mm}^{3}$

2.157 to $30.662^{\circ}$

$-10 \leq h \leq 10,-26 \leq k \leq 26,-27 \leq l \leq 27$

60080

$16299[R(\mathrm{int})=0.0448]$

12479

$99.90 \%$

Multi-scan

0.7461 and 0.6547

Full-matrix least-squares on $F^{2}$

16299 / 8 / 688

1.041

$R 1=0.0393, w R 2=0.0833$

$R 1=0.0622, w R 2=0.0916$

0.565 and -0.529 e. $\AA^{-3}$ 
A thermal ellipsoid plot (ellipsoid contour $50 \%$ probability) of the crystal structure.

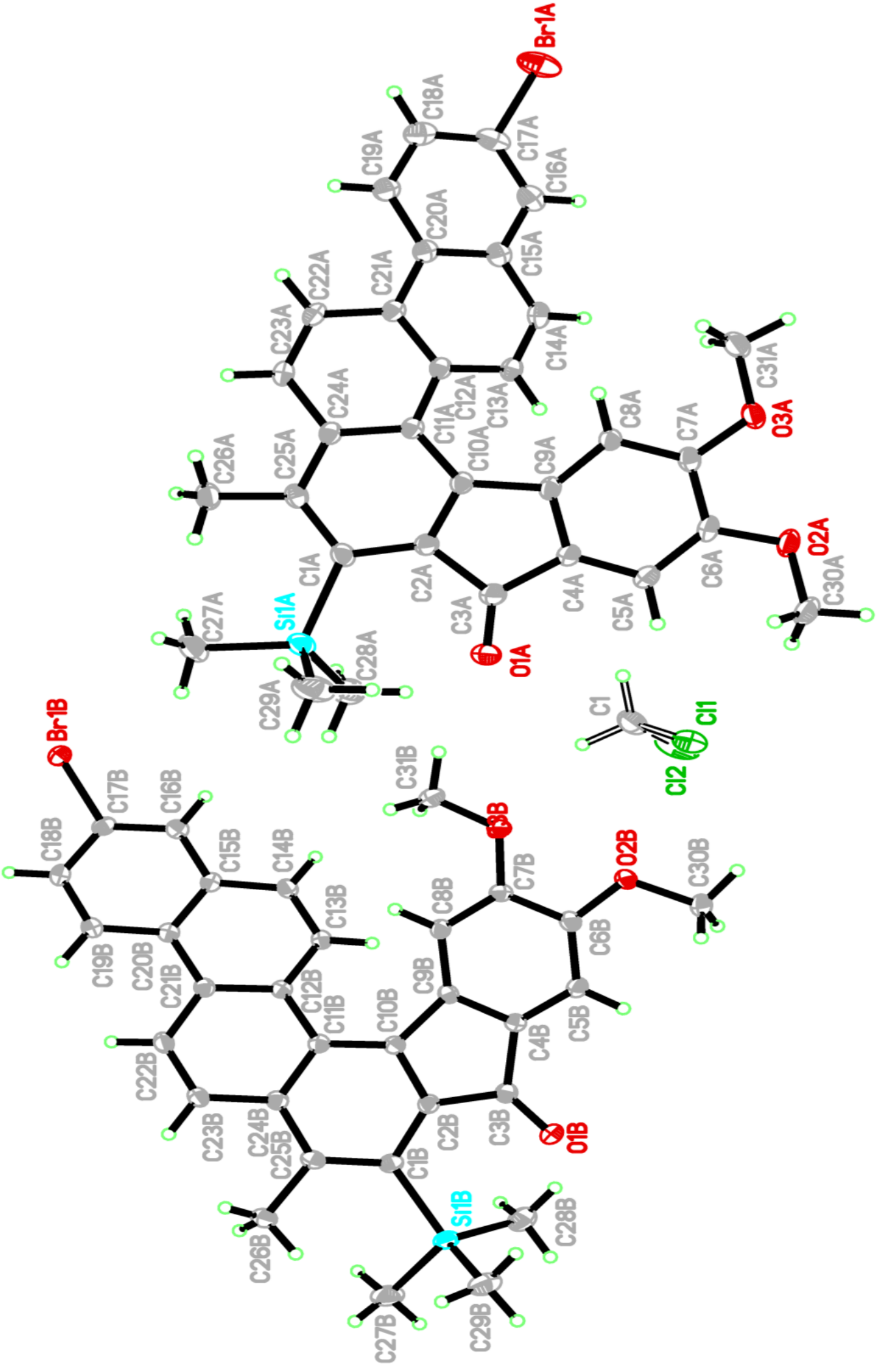




\section{References for the Supplementary Information}

1 Hoye, T. R.; Hanson, P. R.; Vyvyan, J. R. A practical guide to first-order multiplet analysis in ${ }^{1} \mathrm{H}$ NMR spectroscopy. J. Org. Chem. 1994, 59, 4096-4103.

2 Hoye, T. R.; Zhao, H. A method for easily determining coupling constant values: an addendum to "a practical guide to first-order multiplet analysis in " $\mathrm{H}$ NMR spectroscopy". J. Org. Chem. 2002, 67, 4014-4016.

3 From the Supporting Information of Xiao, X.; Hoye, T. R. The domino hexadehydro-Diels-Alder reaction transforms polyynes to benzynes to naphthynes to anthracynes to tetracynes (and beyond?). Nature Chemistry 2018, 10, 838-844.

4 Beltran, F.; Miesch, L. Org. Lett. 2019, 21, 1569-1573.

5 Pigulski, B.; Arendt, A.; Tomilin, D. N.; Sobenina, L. N.; Trofimov, B.A.; Szafert, S. J. Org. Chem. 2016, 81, 9188-9198.

6 Chinta, B. S.; Baire, B. Org. Biomol. Chem. 2018, 16, 262-265.

7 Beltran, F.; Fabre, I.; Ciofini, I.; Miesch, L. Org. Lett. 2017, 19, 5042-5045.

8 Hoye, T.R.; Sahil, A.; Vedamayee, P. Chem. Sci. 2019, 10, 9069-9076.

9 Álvarez-Calero, J. M.; Jorge, Z. D.; Massanet, G. M. Org. Lett. 2016, 18, 6344-6347.

10 Zadlo, A.; Koszelewski, D.; Borys, F.; Ostaszewski, R. Chem. Bio. Chem. 2015, 16, 677-682.

11 Wei, J.; Wang. P.; Jia, Q.; Huang, J.; Du, Z.; Zhang, K.; Wang, J. Eur. J. Org. Chem. 2013, 44994502.

12 Kosiova, I.; Kovackova, S.; Kois P. Tetrahedron 2007, 63 312-320.

13 Kutubi, S, Md.; Hashimoto, T.; Kitamura, T. Synthesis 2011, 8, 1283-1289.

14 Hon, Y-S.; Hong, Y-C.; Hong, B-C.; Liao, J-H. J. Chin. Chem. Soc. 2012, 59, 407-420.

15 Lakshmi, B. V.; Wefelscheid, U. K.; Kazmaier, U. Synlett 2011, 3, 0345-0348.

16 Sheldrick, G. M. A short history of SHELX. Acta Cryst. A64, 112-122 (2008). 


\section{Copies of ${ }^{1} \mathrm{H},{ }^{13} \mathrm{C}, 2$-D NMR spectra}




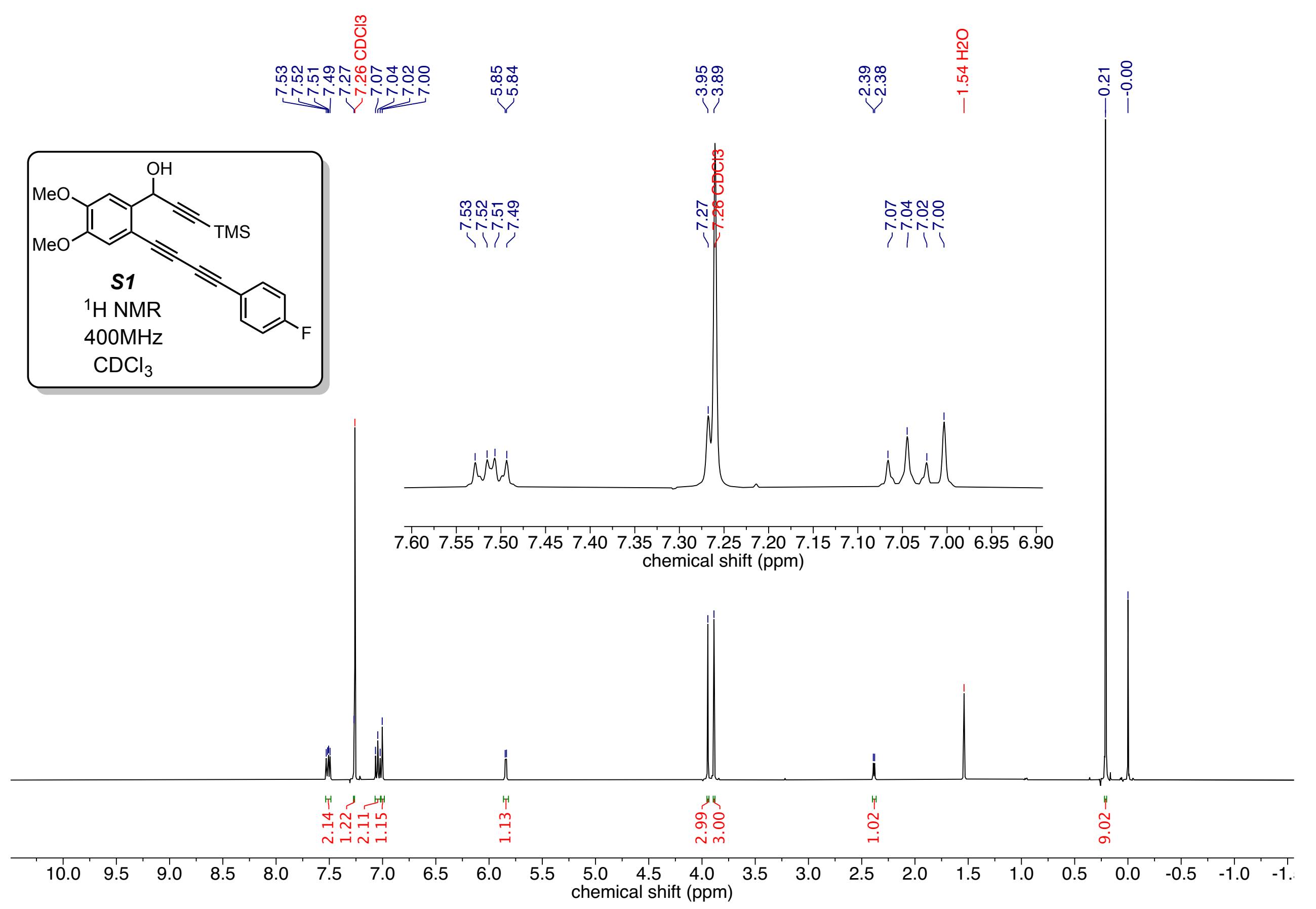



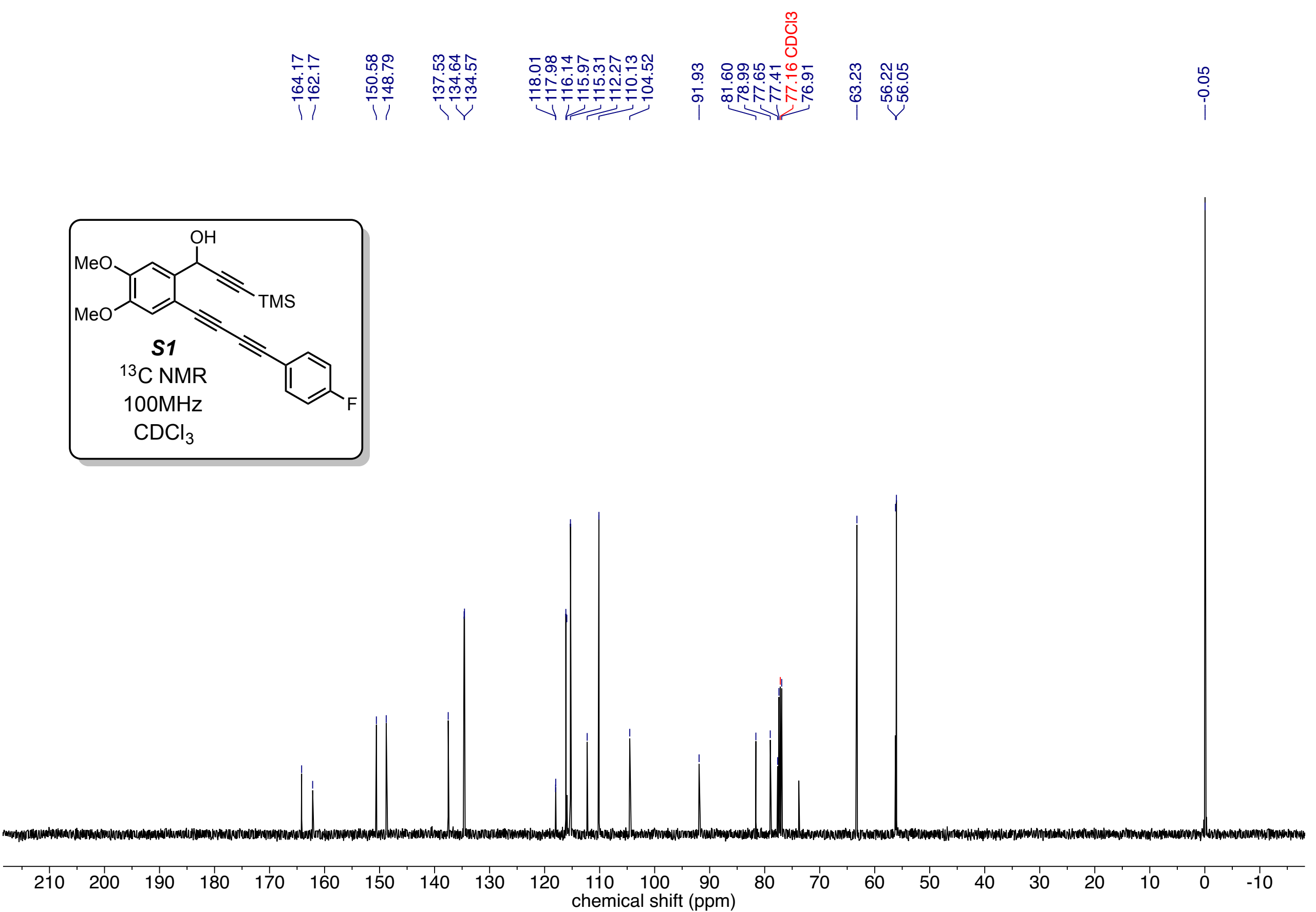


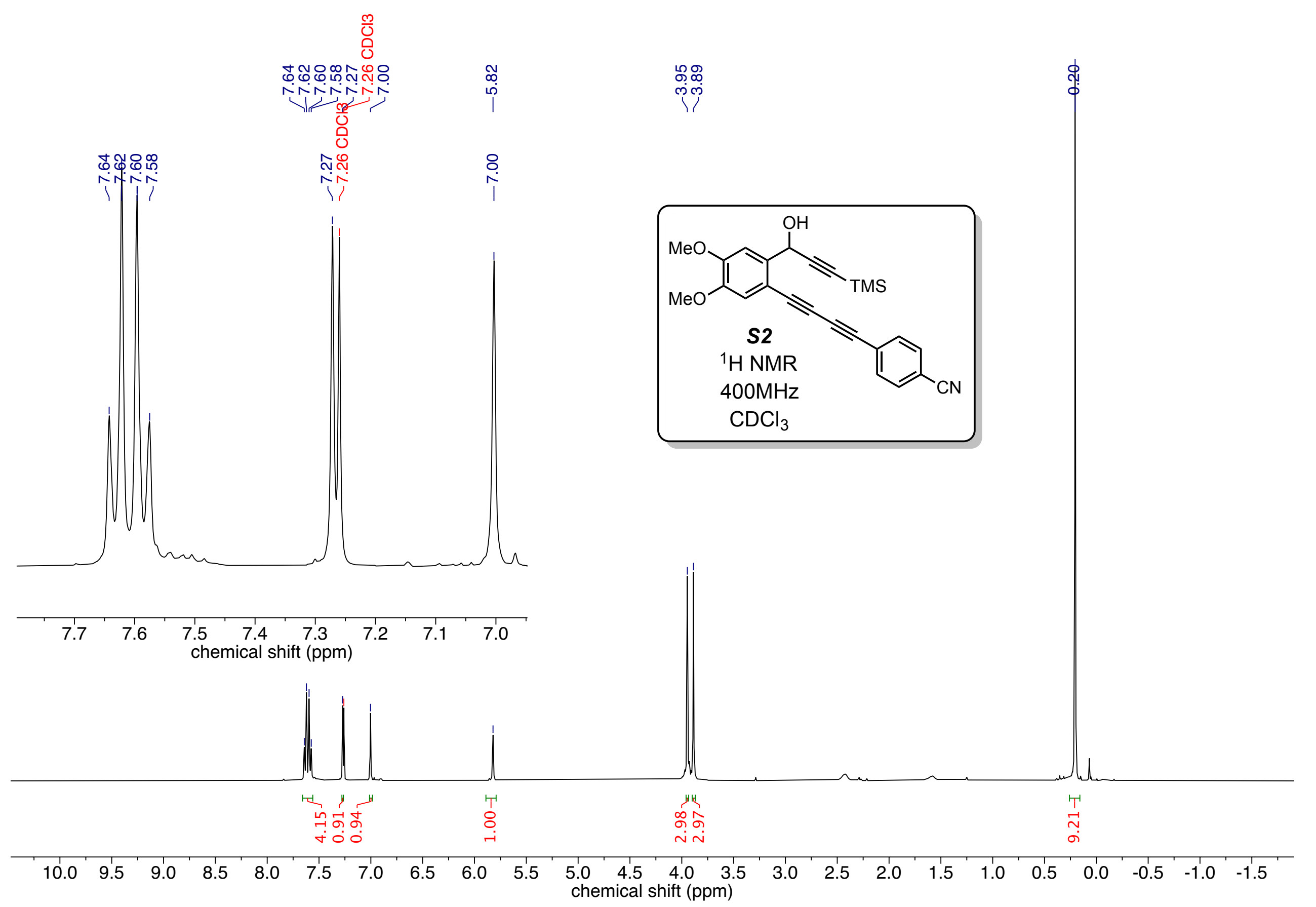




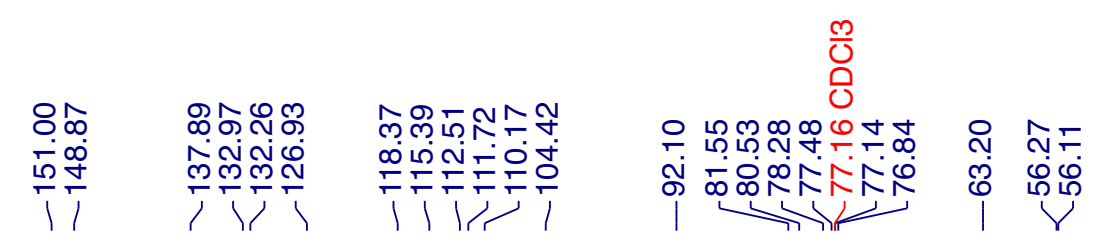

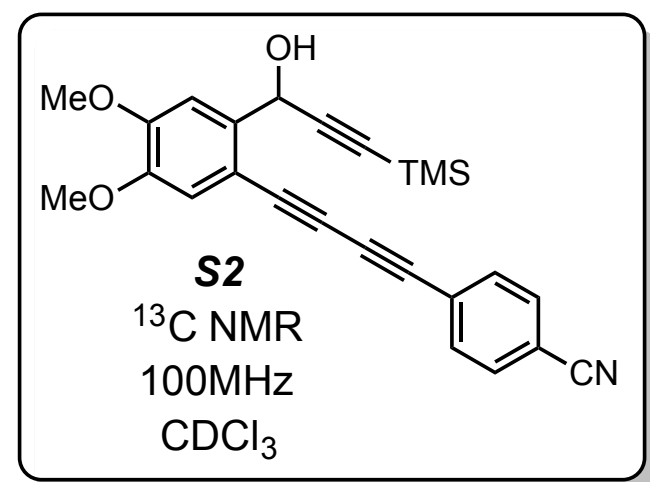




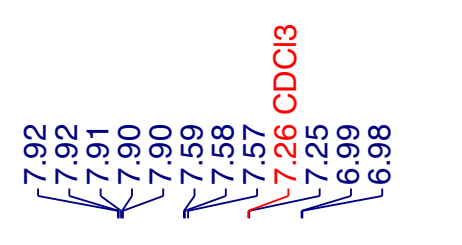

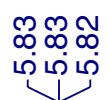
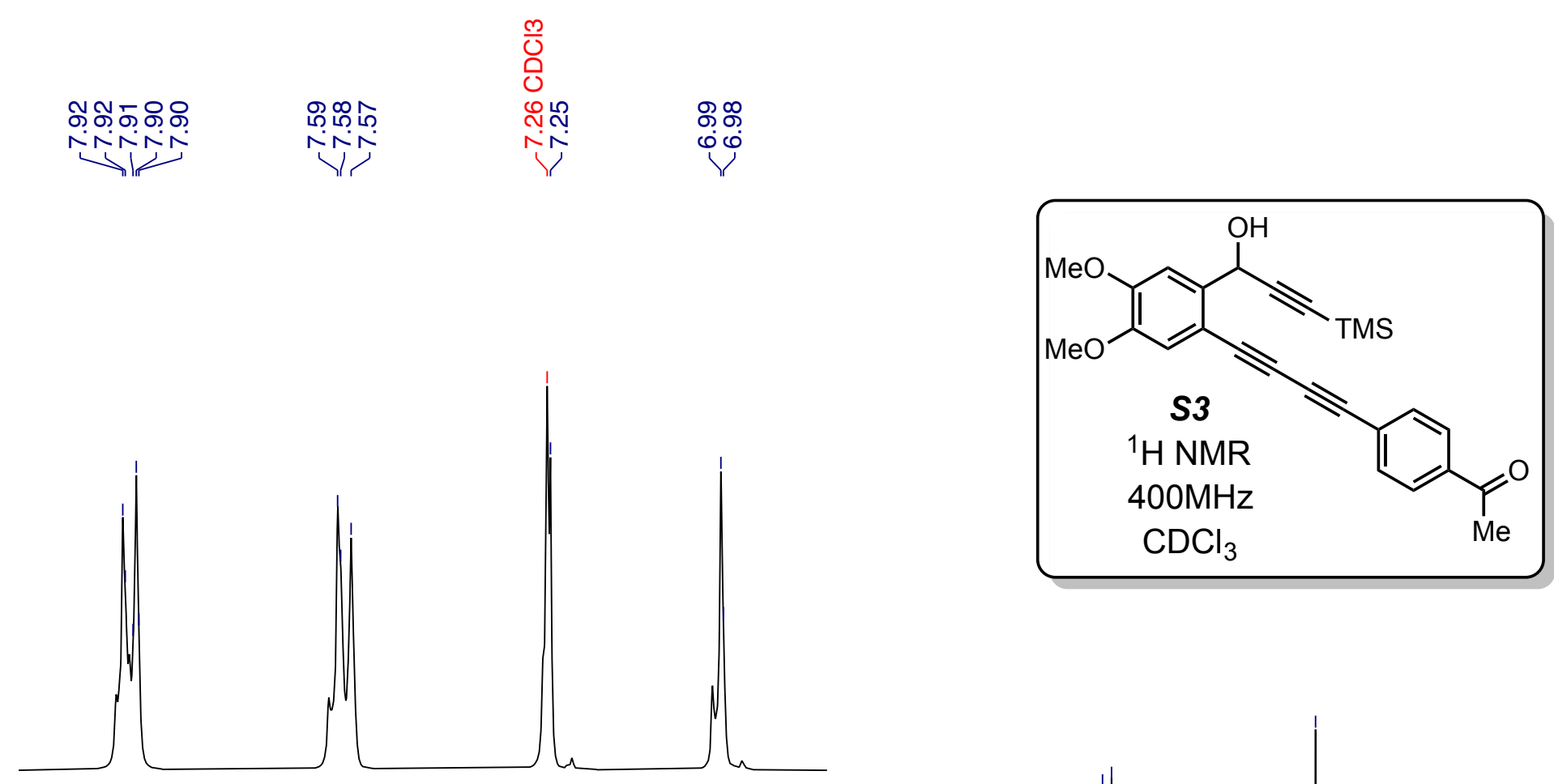

$\begin{array}{llllllllllll}8.0 & 7.9 & 7.8 & 7.7 & 7.6 & 7.5 & 7.4 & 7.3 & 7.2 & 7.1 & 7.0 & 6.9\end{array}$
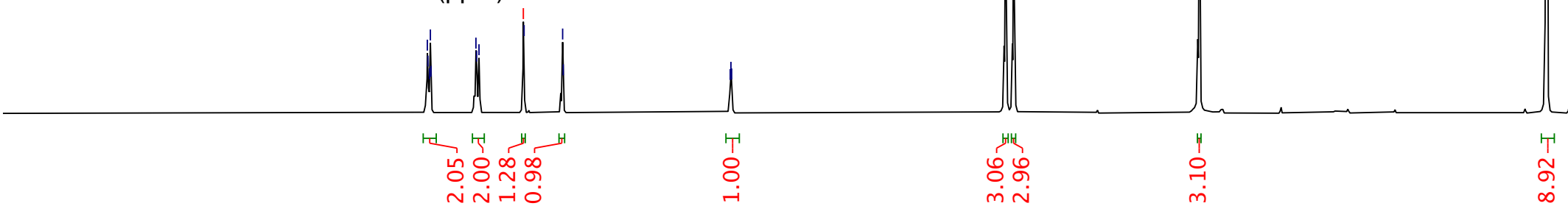

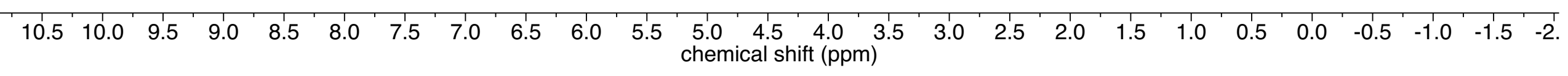




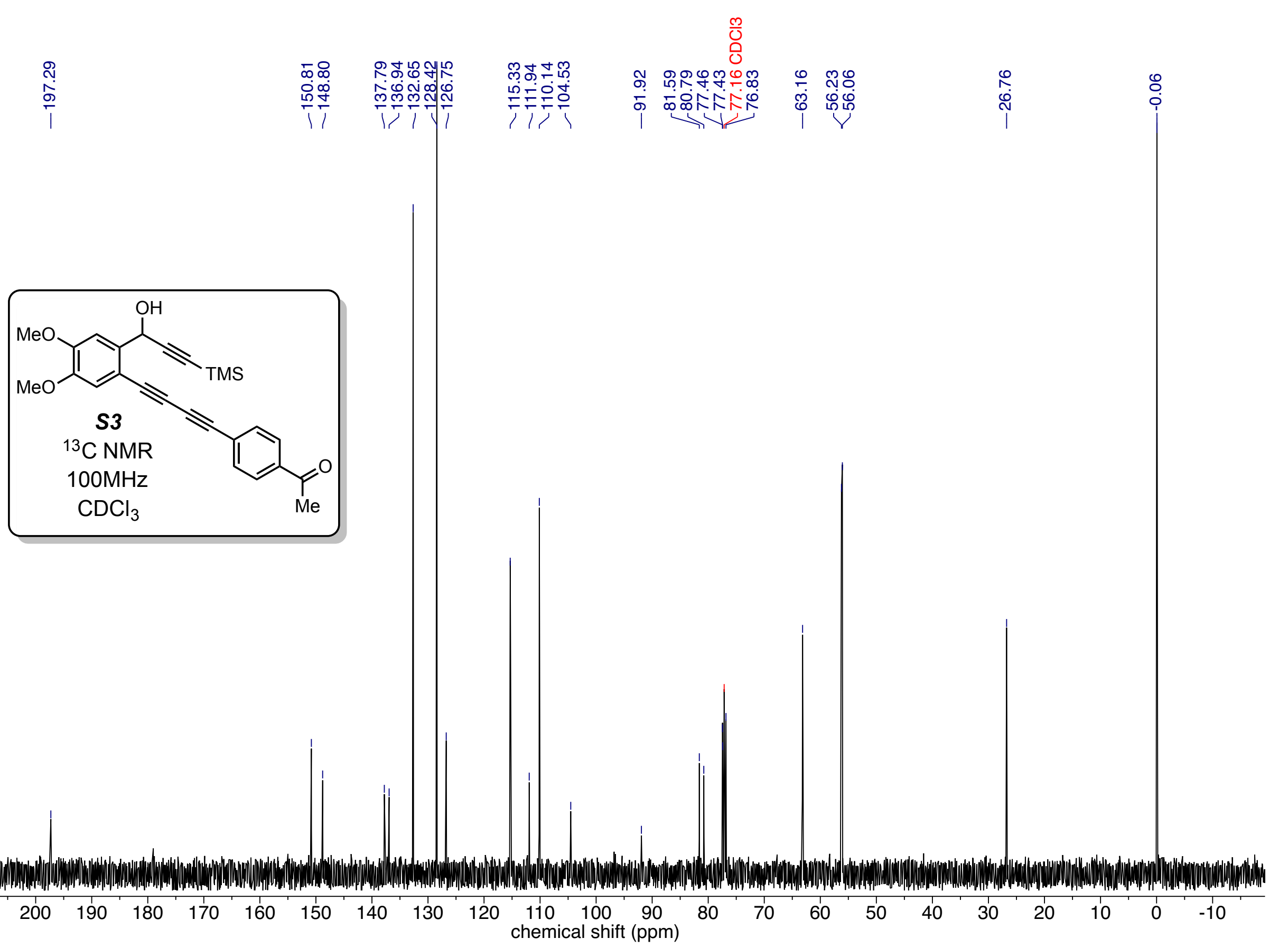



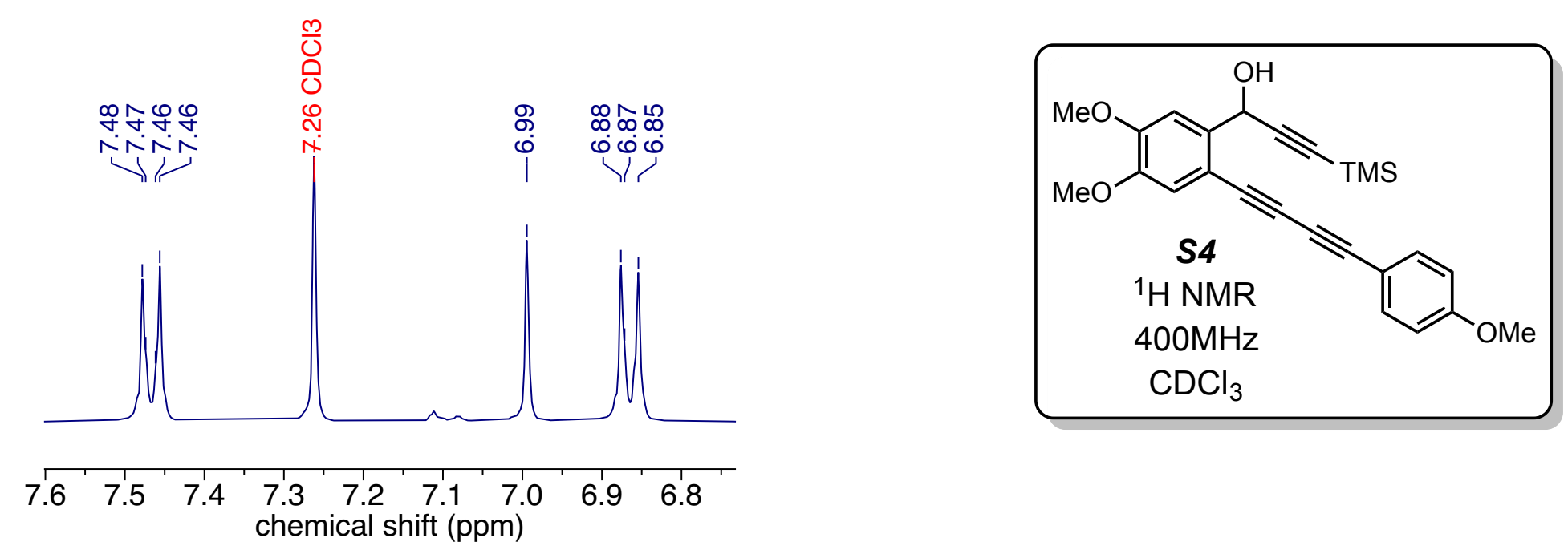

\section{पె口}

लंm

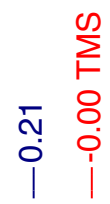

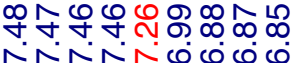

诲inivio

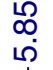

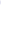

पा?

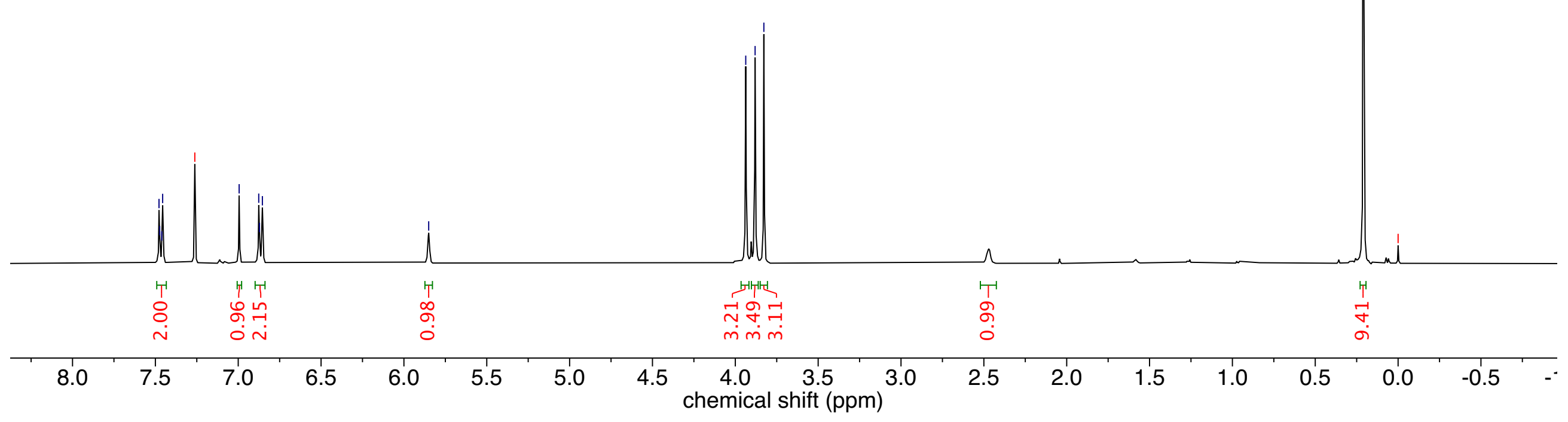




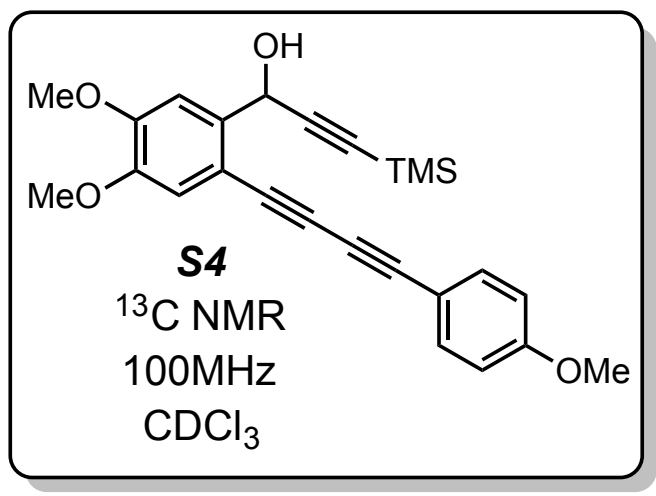

\section{W}

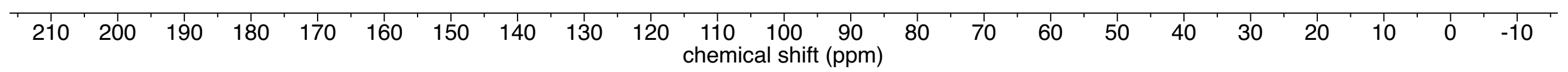


$\frac{m}{0}$
ปे

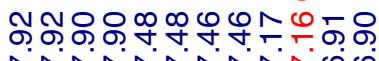

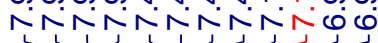

ำ

นึ่

模

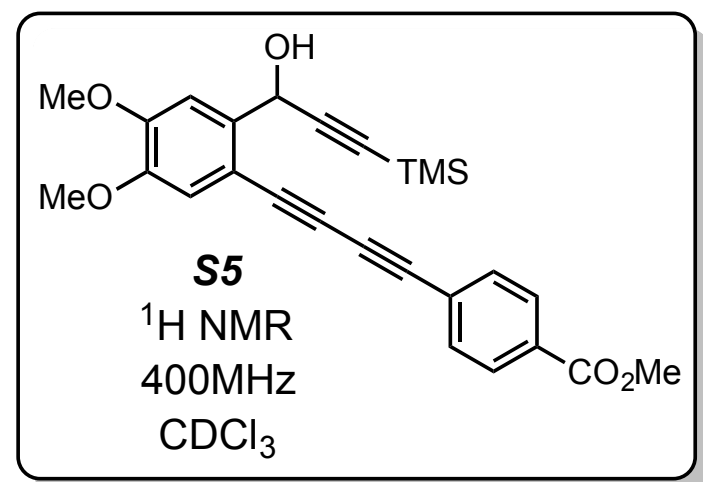

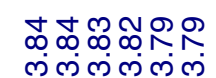

लुलूल्य
퉁우

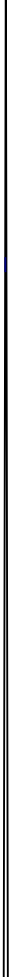

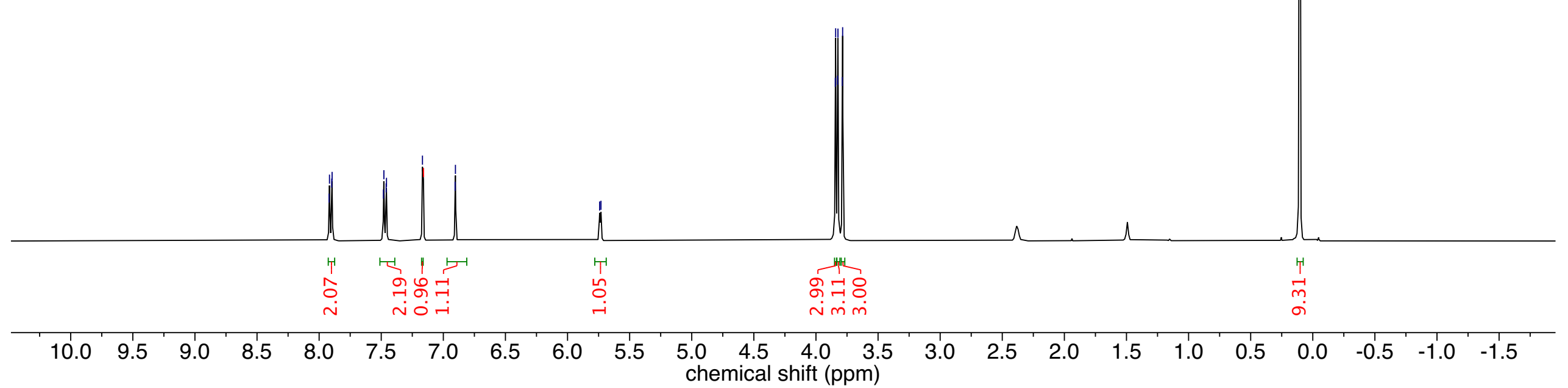




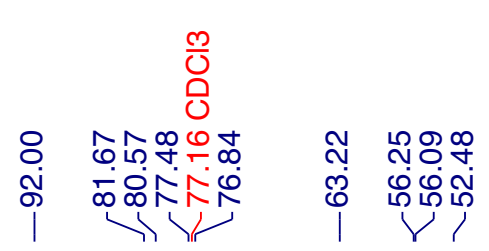
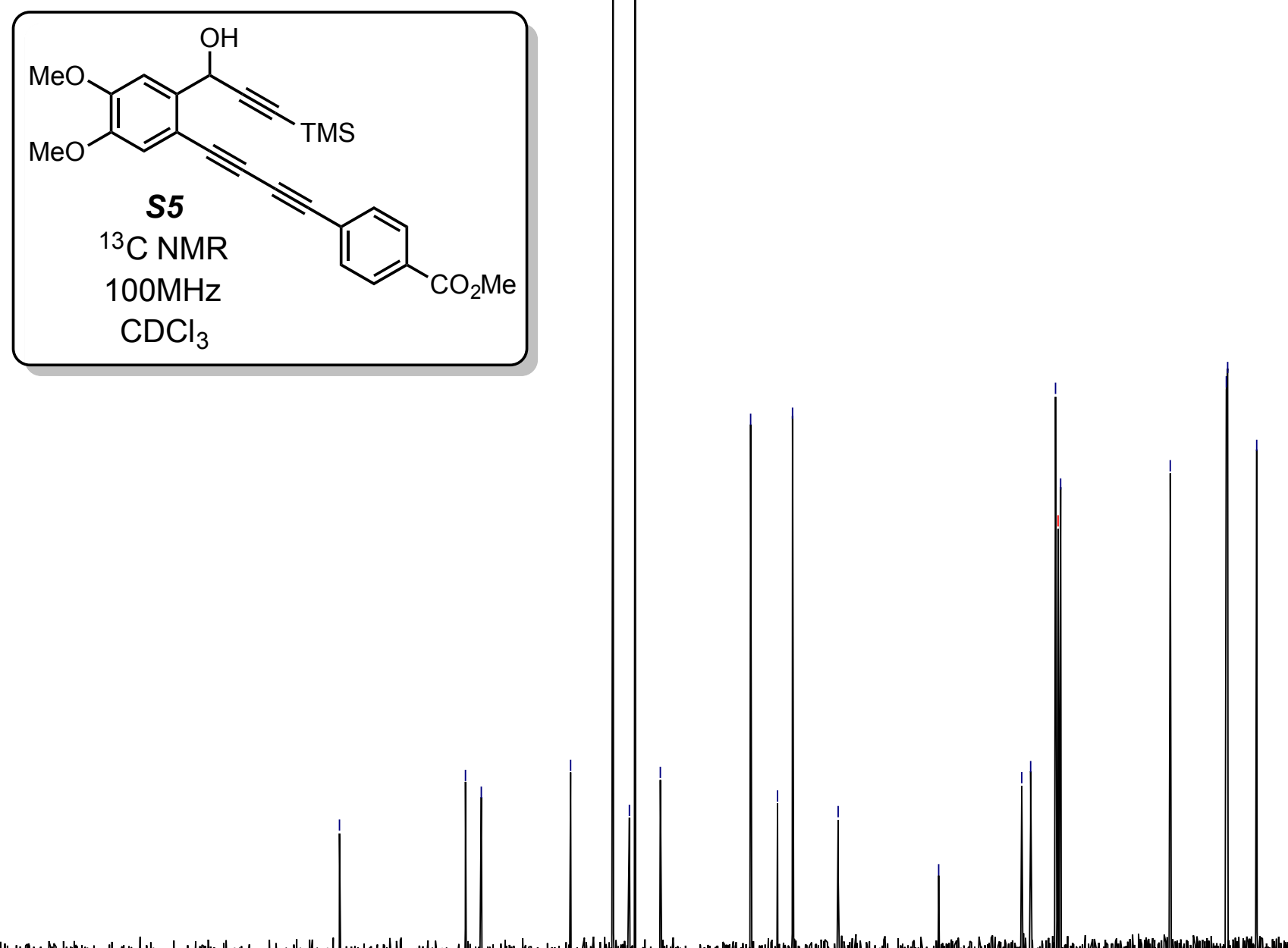


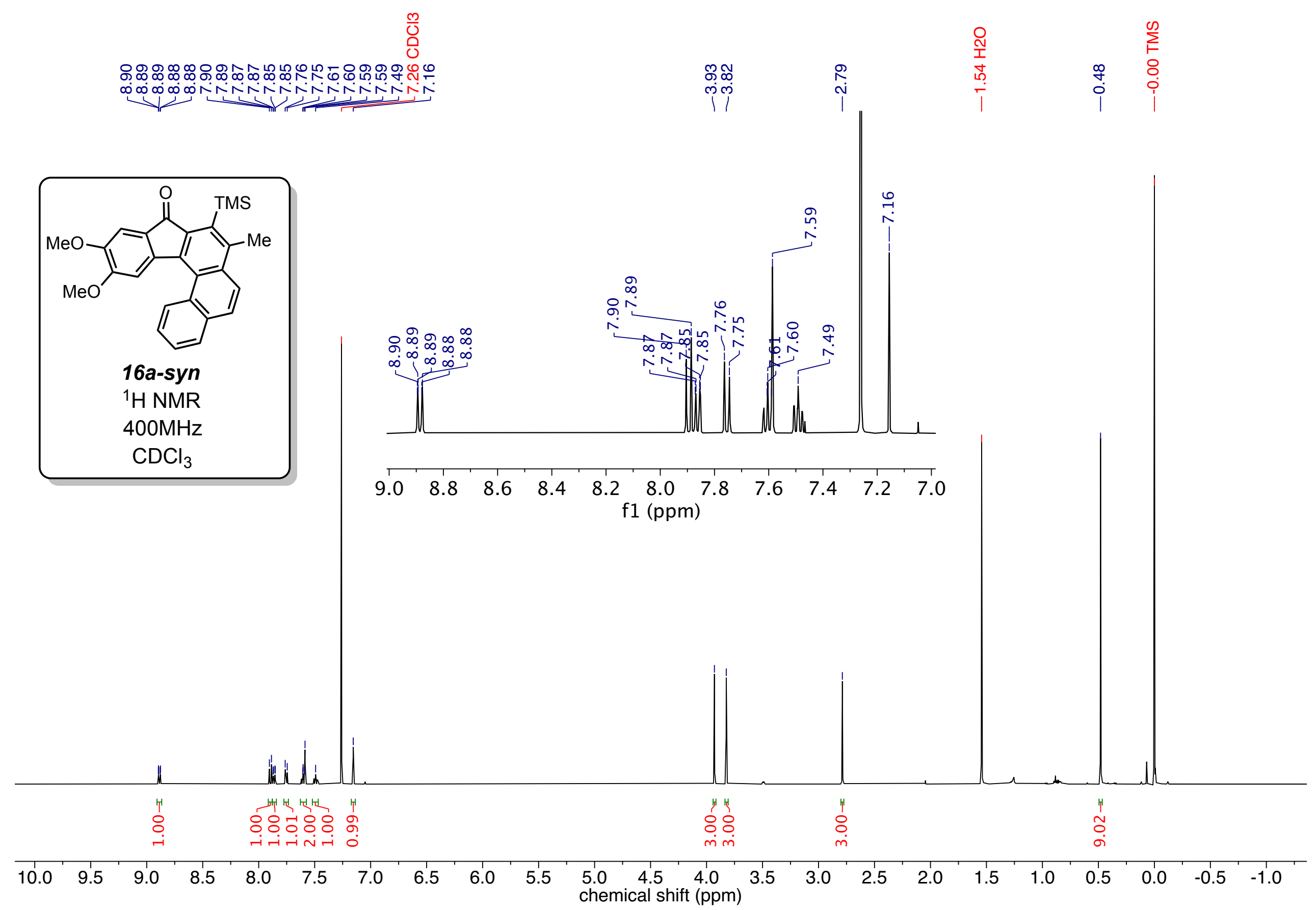



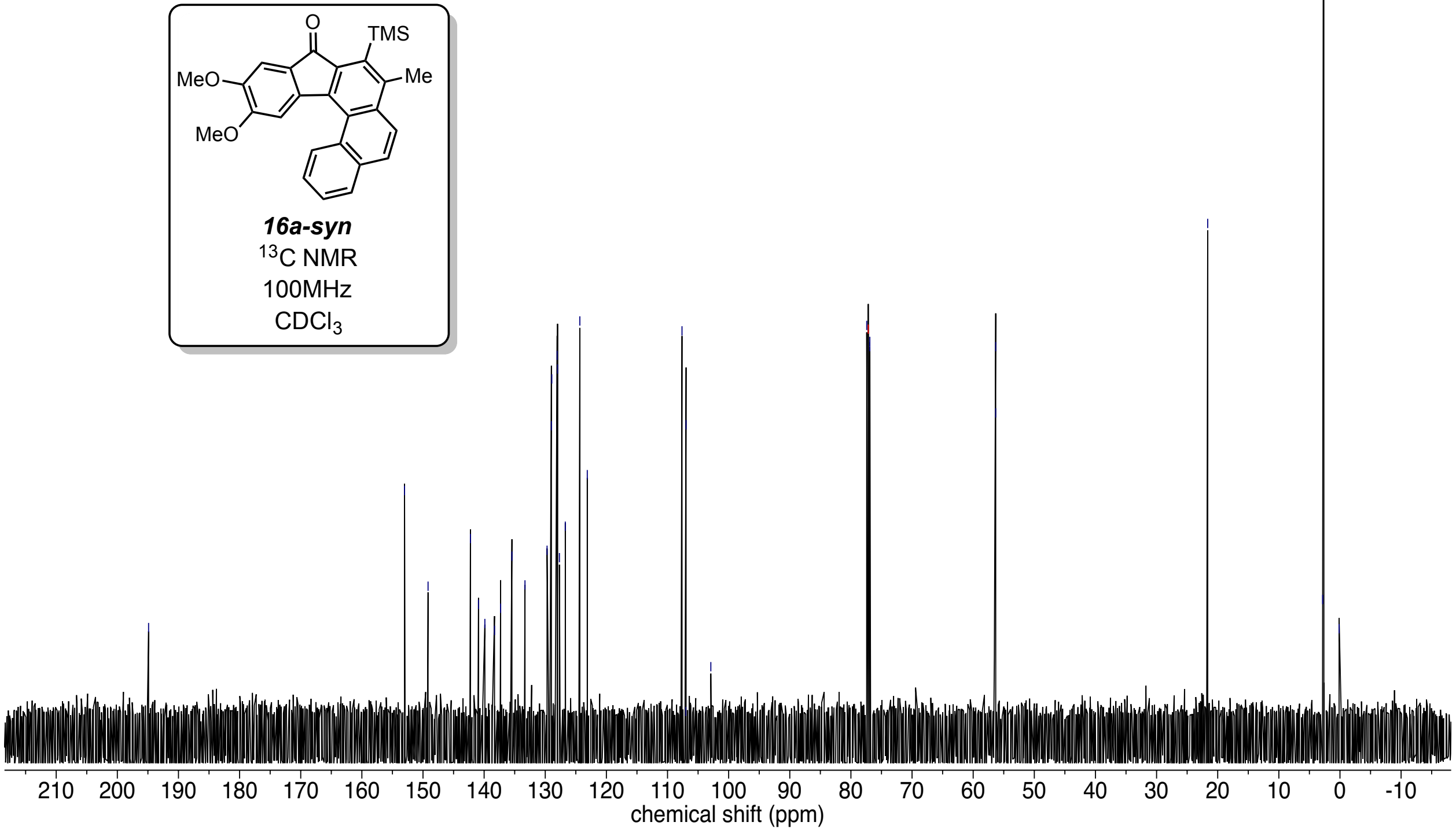


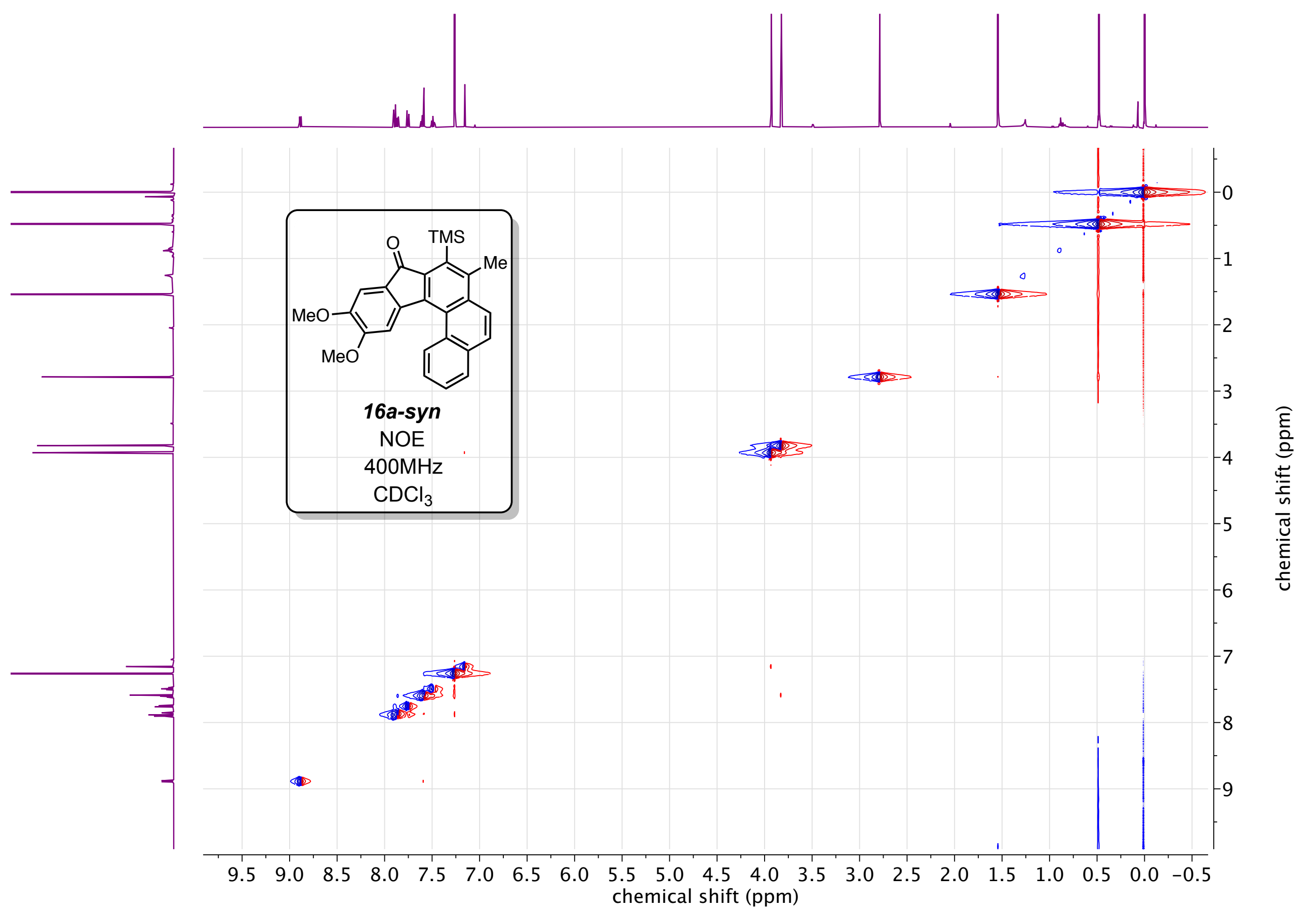




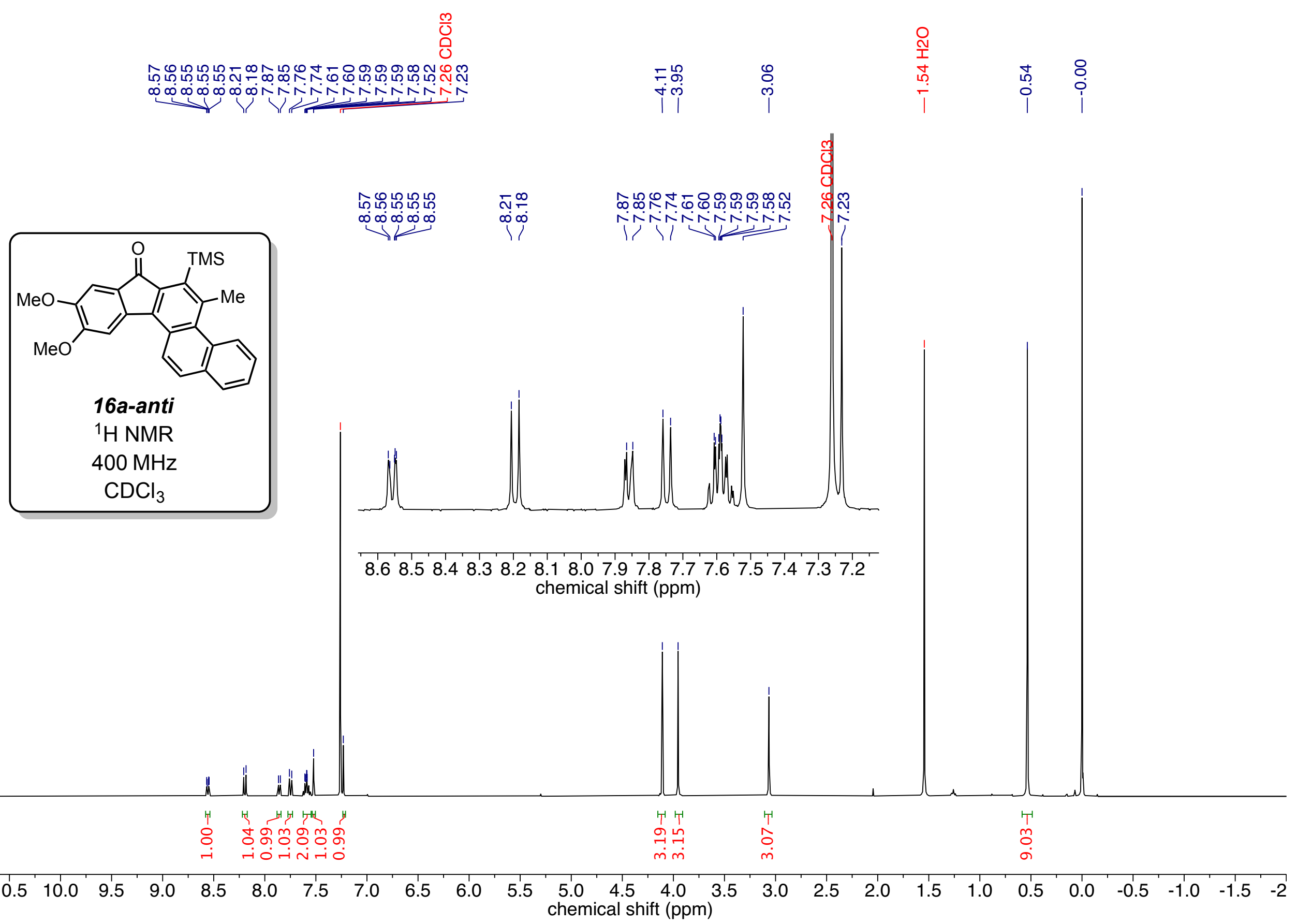



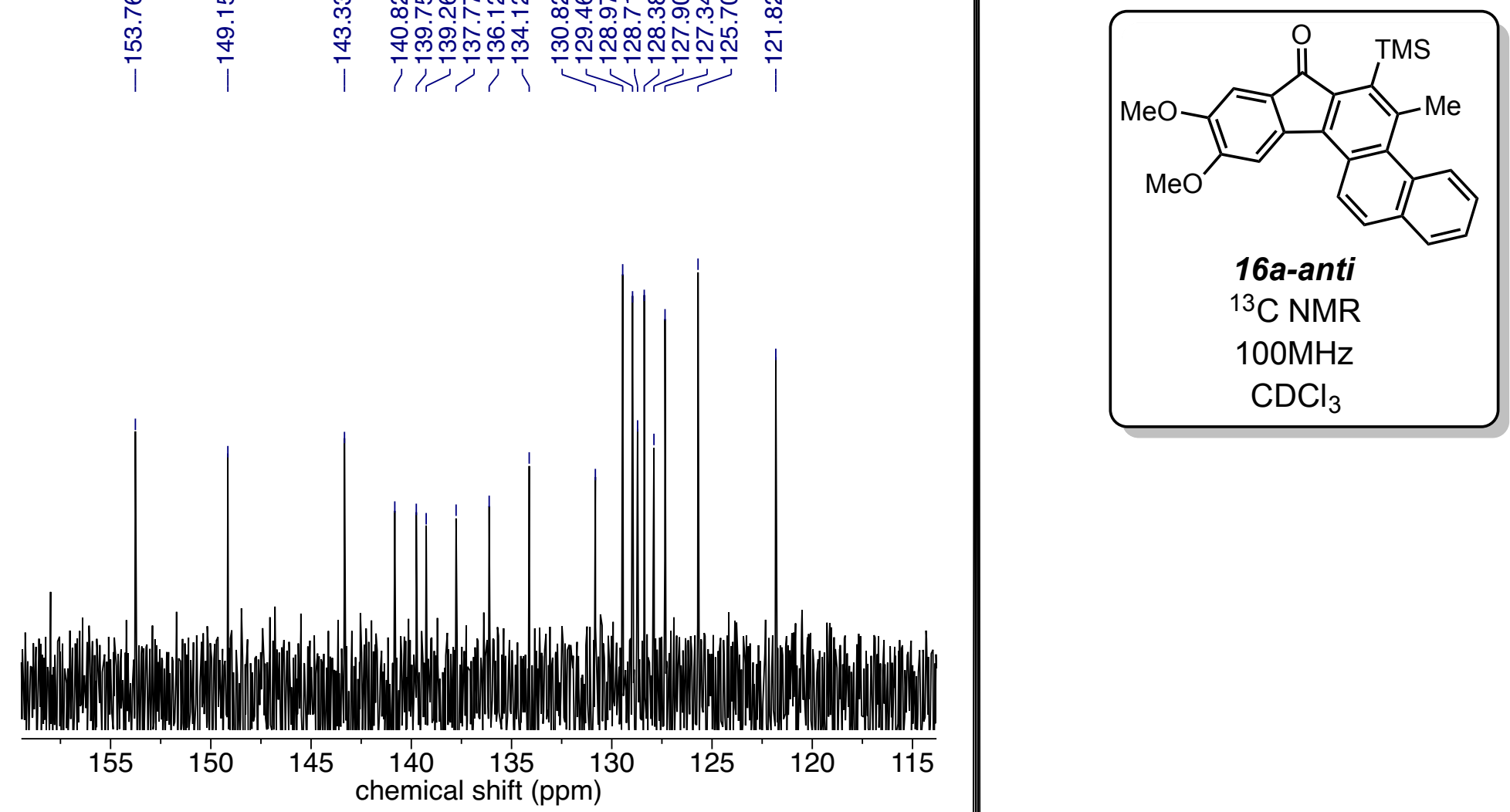


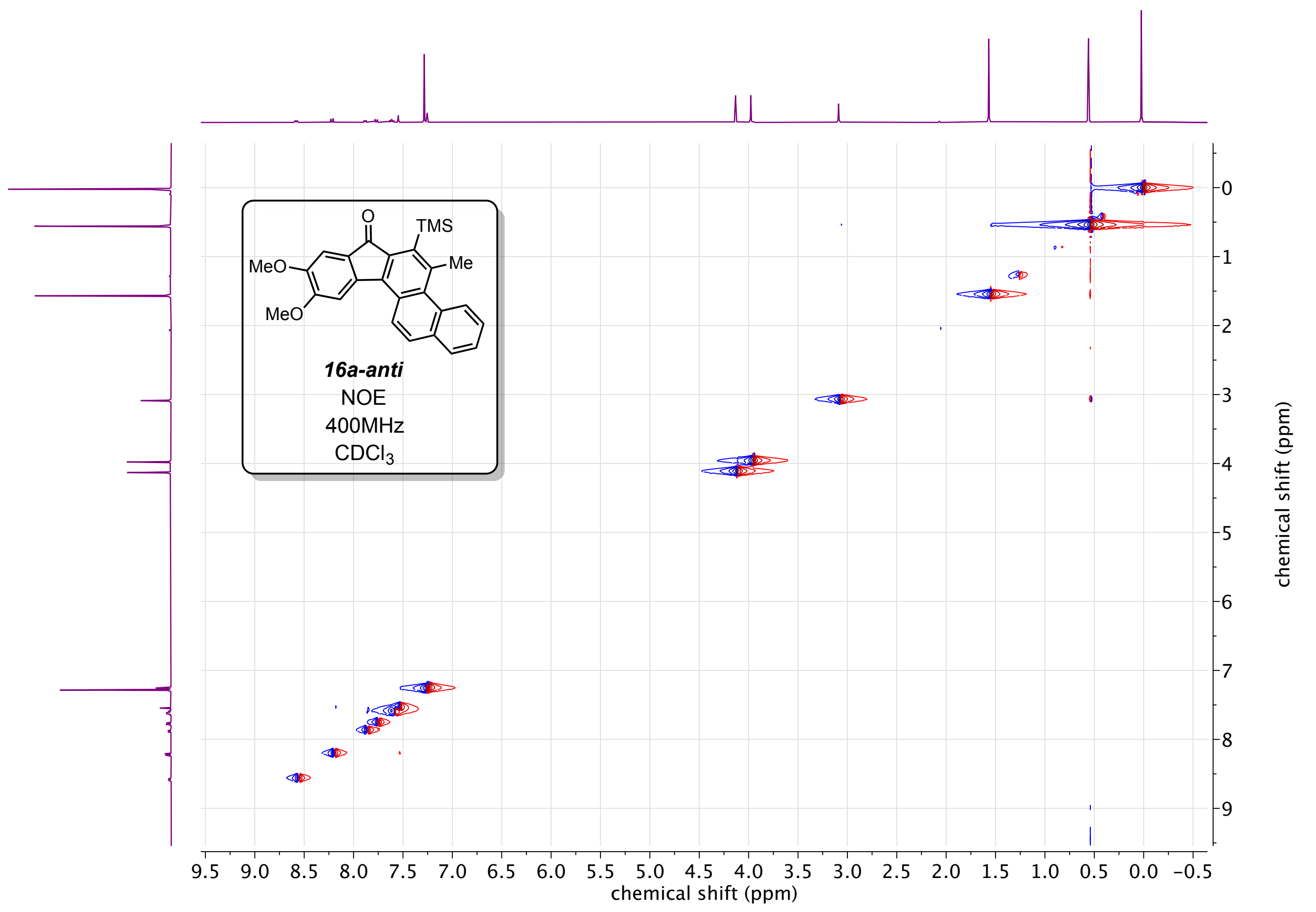



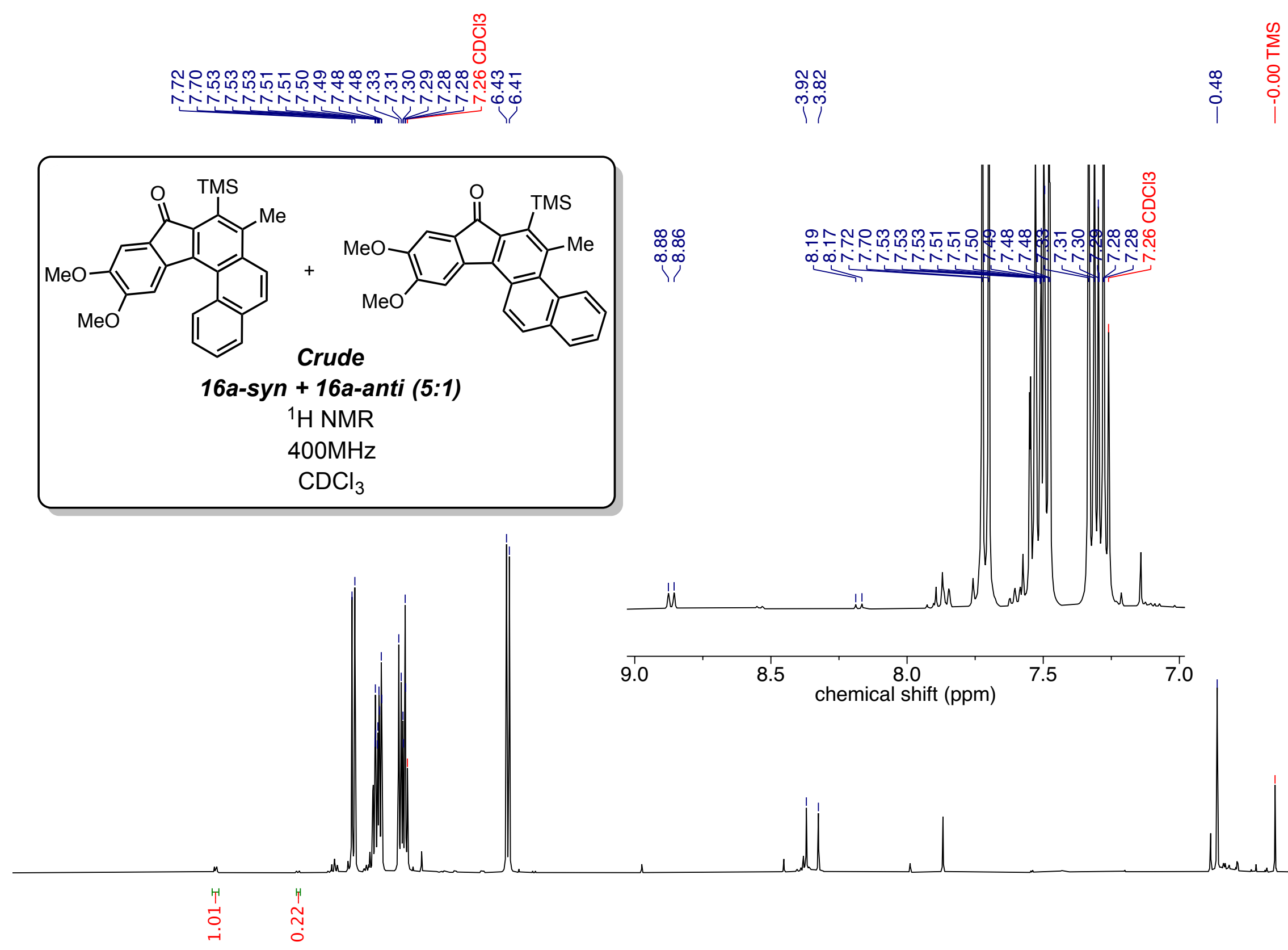


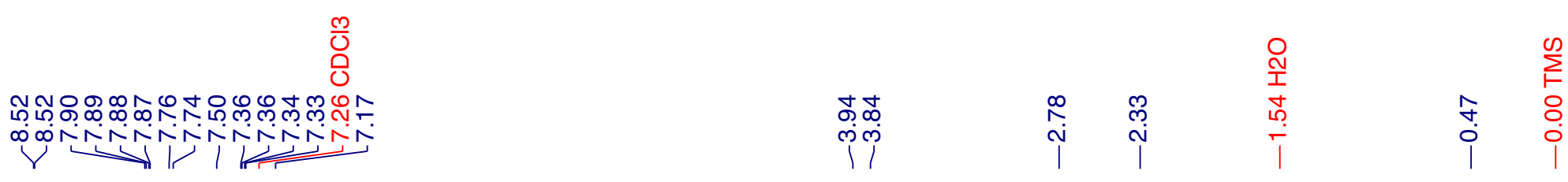
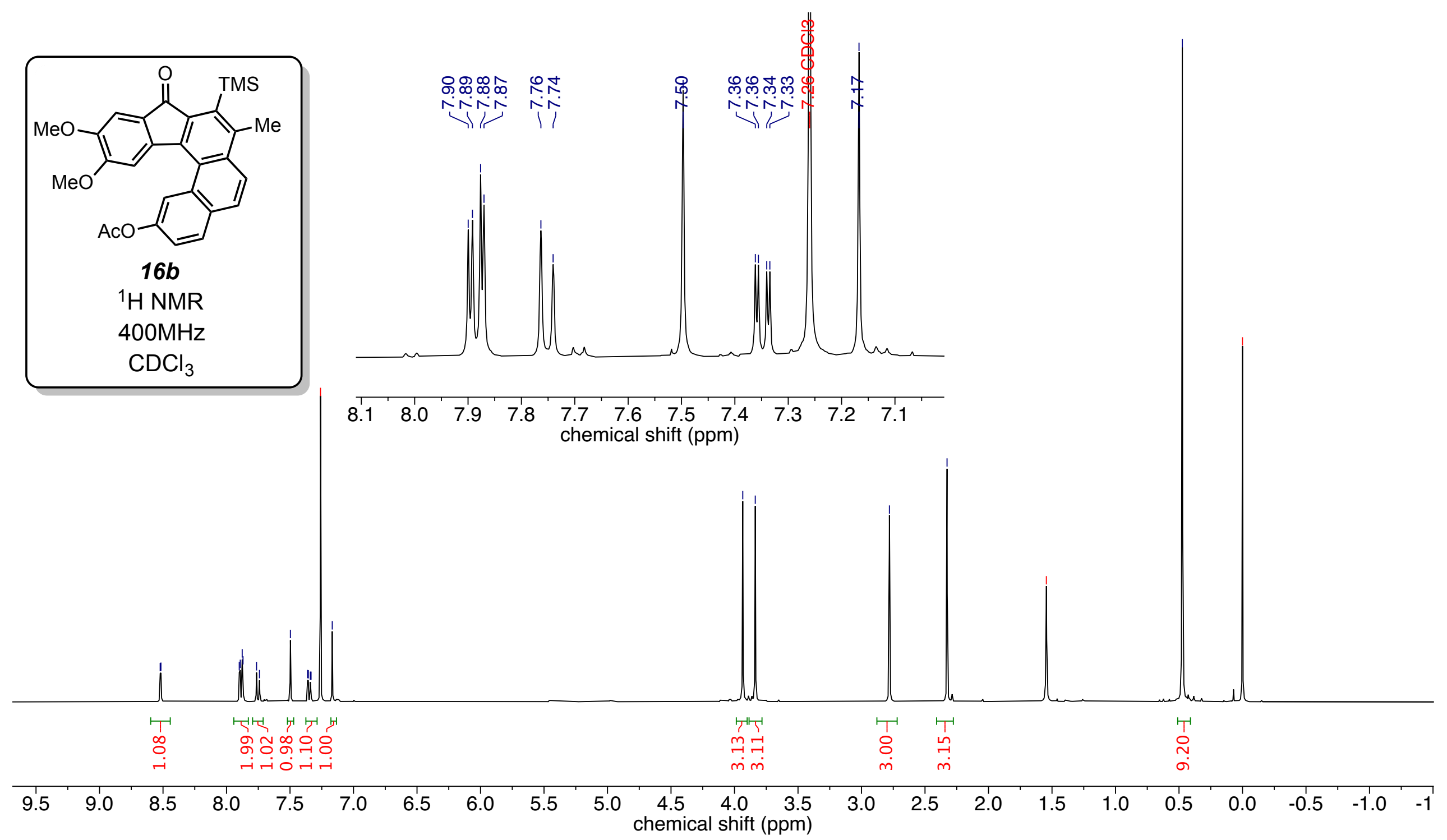


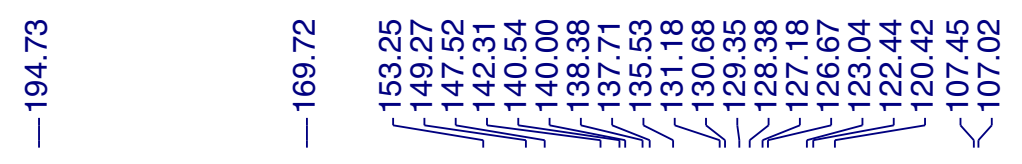

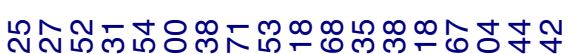

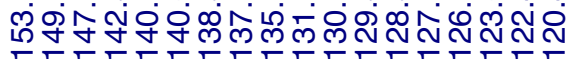
个,

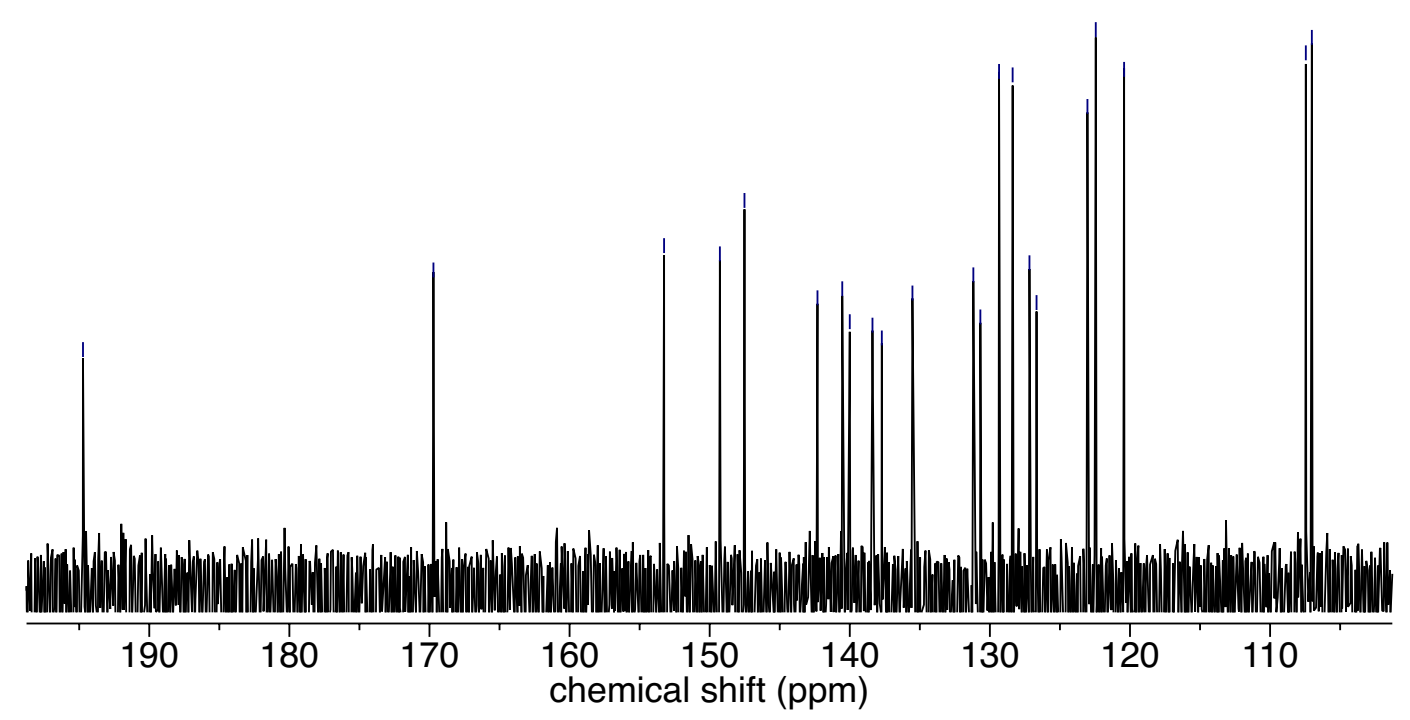

m户

它

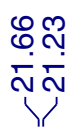

厄ே.

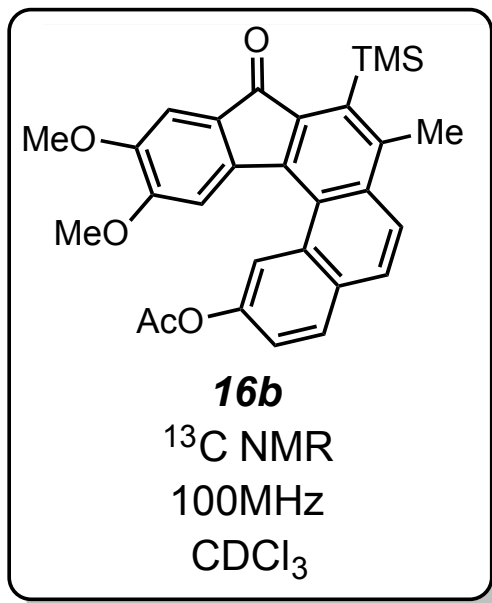



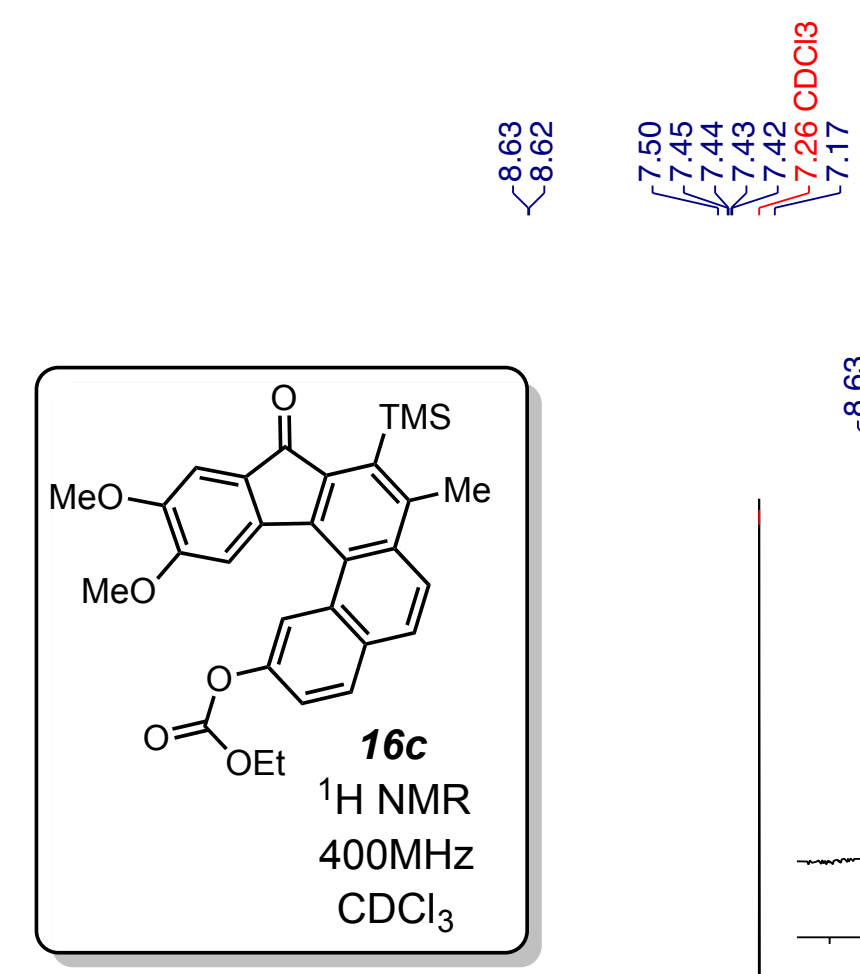

要

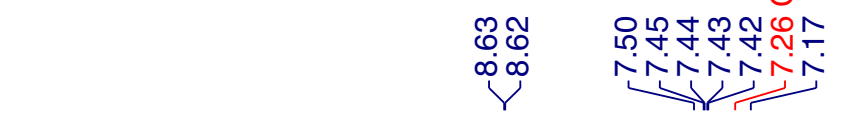

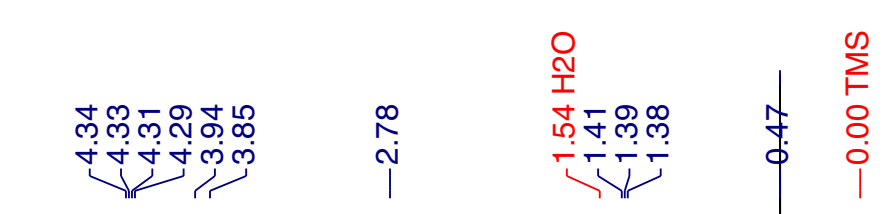

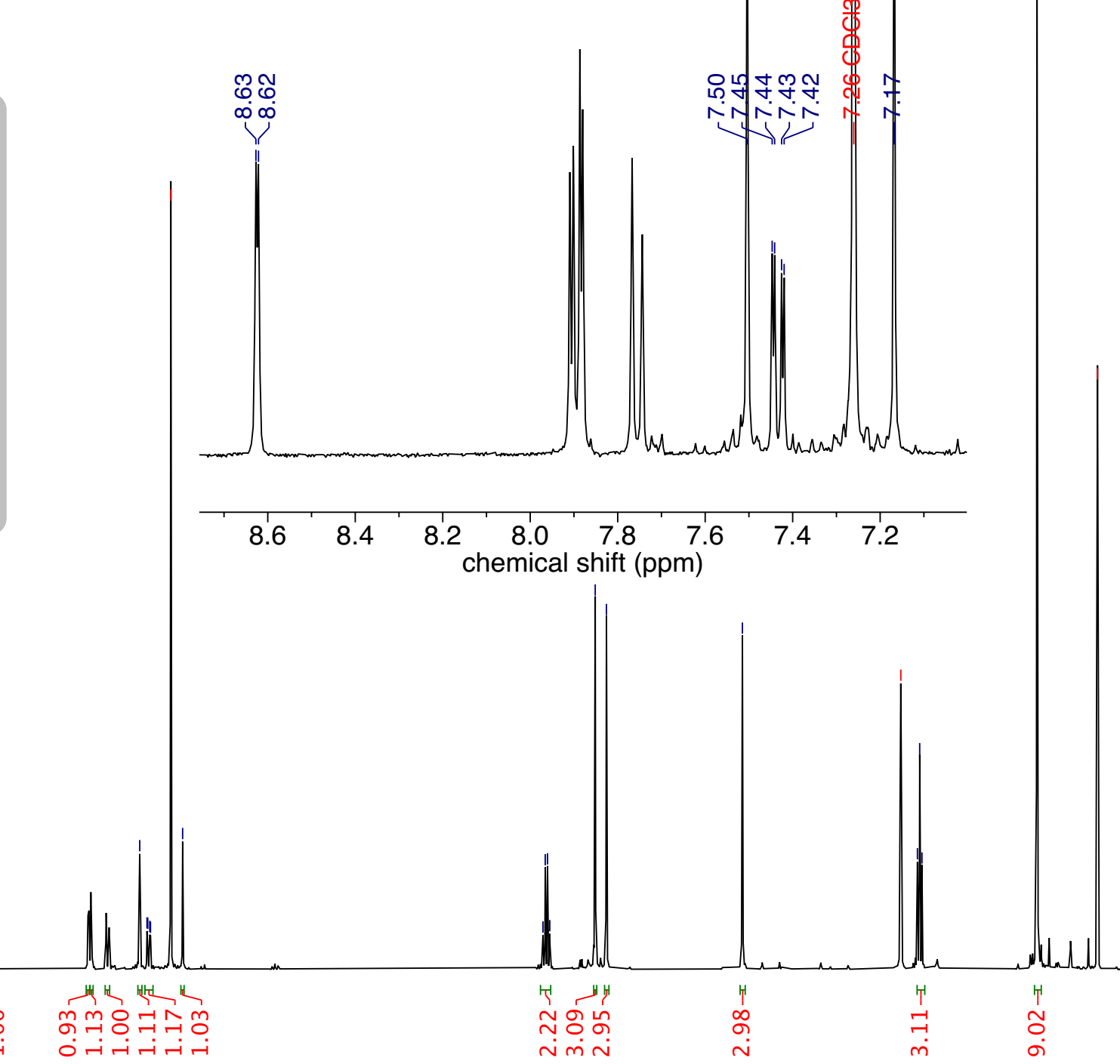

$\begin{array}{llllllllllllllllllllllllllllllllllllllll}11.5 & 11.0 & 10.5 & 10.0 & 9.5 & 9.0 & 8.5 & 8.0 & 7.5 & 7.0 & 6.5 & 6.0 & 5.5 & 5.0 & 4.5 & 4.0 & 3.5 & 3.0 & 2.5 & 2.0 & 1.5 & 1.0 & 0.5 & 0.0 & -0.5 & -1.0 & -1.5 & -2.0 & -2.5 & -3 . \\ \text { chemical shift }(\mathrm{ppm})\end{array}$ 


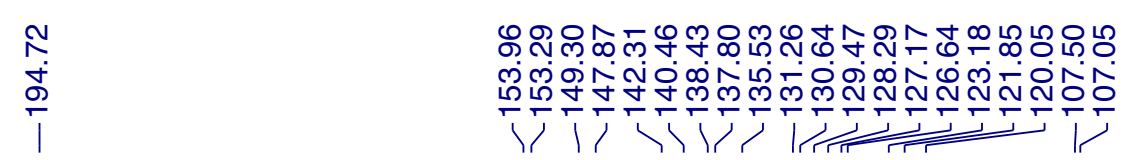

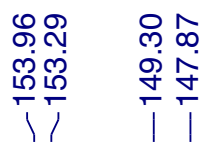
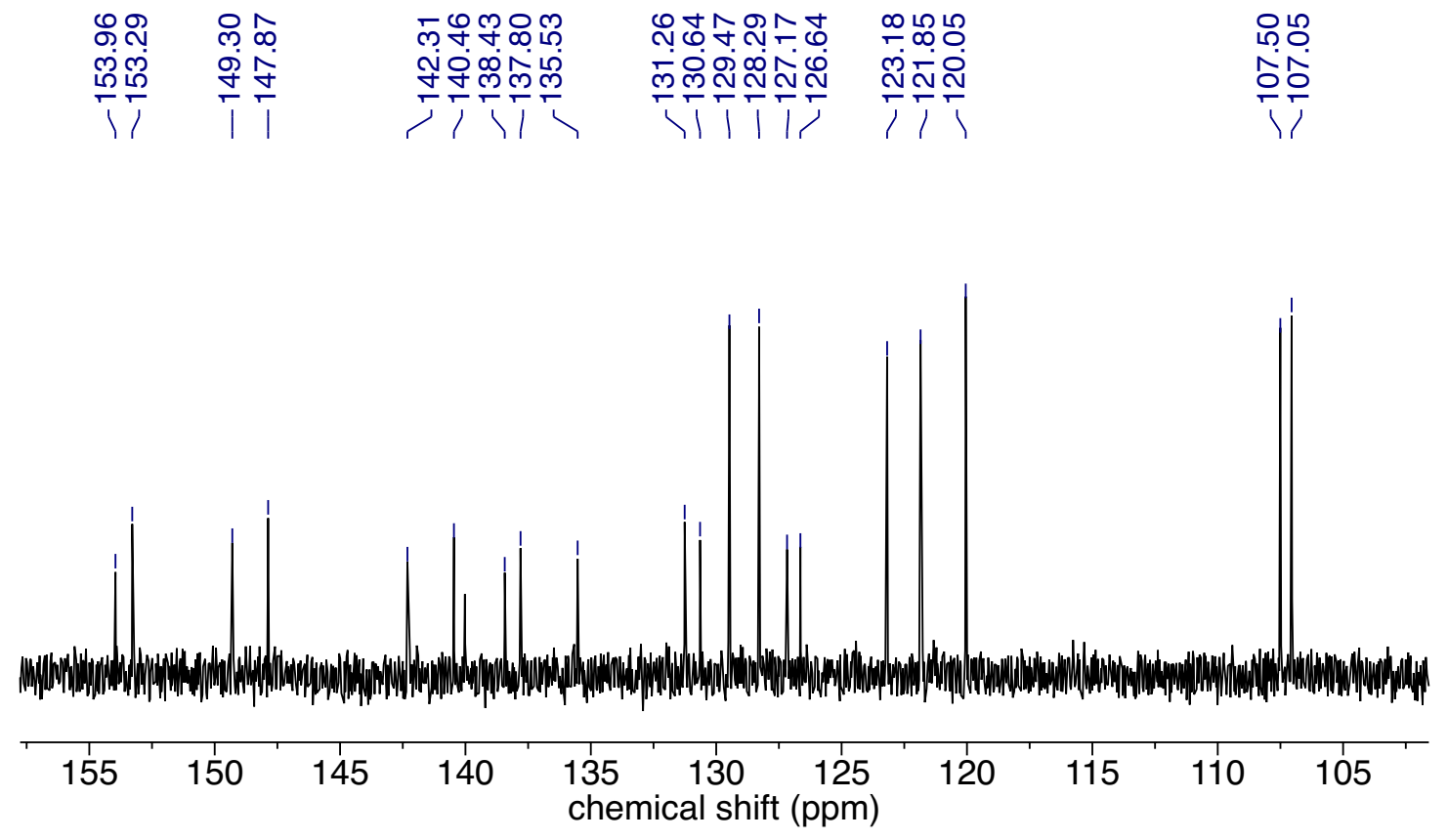

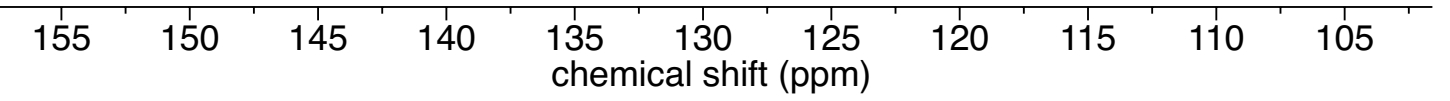

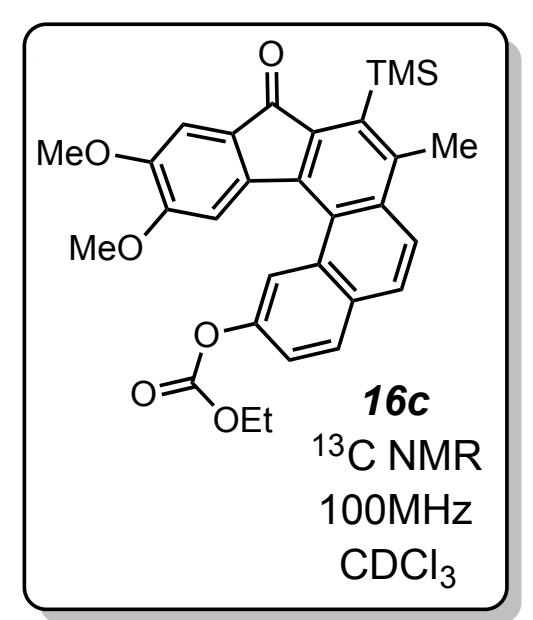

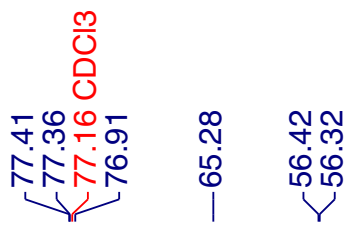

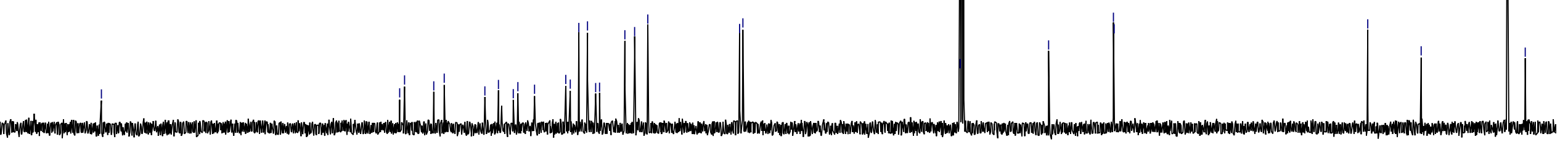


$\frac{m}{0}$

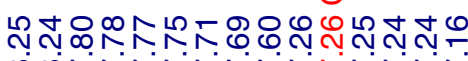

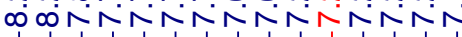

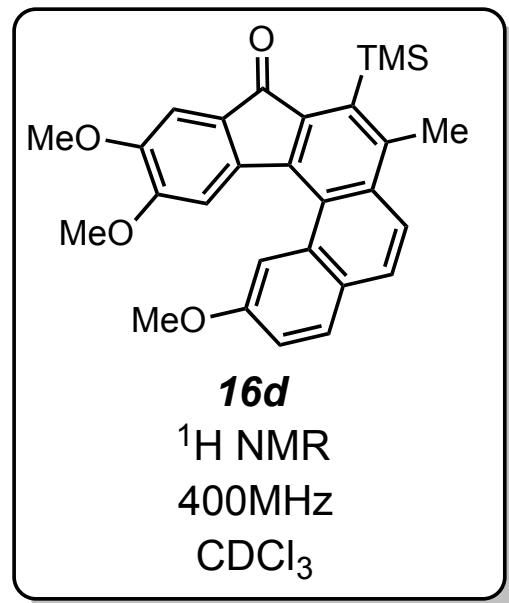

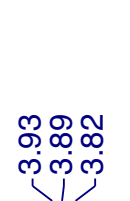

लंगु

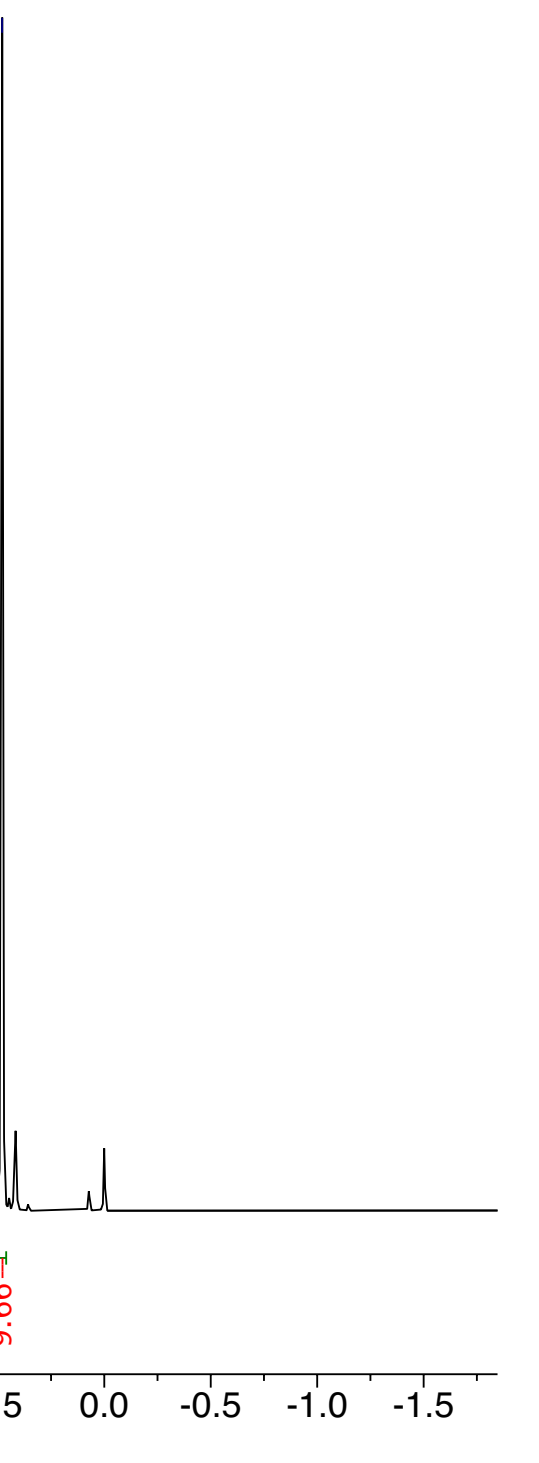




\section{ल유:}

ํํำ

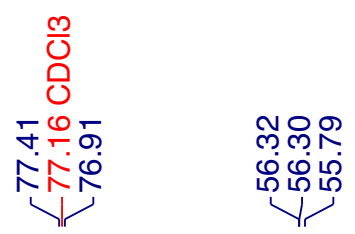

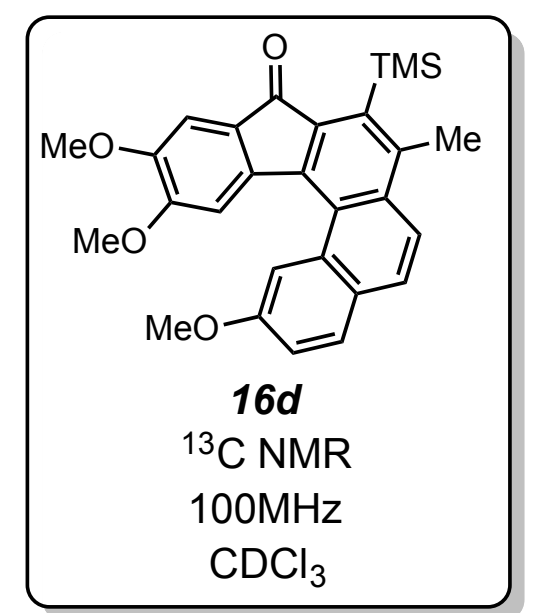

21

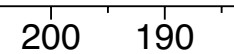

180

170

160

150

$140 \quad 130$

$\begin{array}{lll}120 & 110 & 100\end{array}$

chemical shift (ppm)

80

70

60

50

$40 \quad 30$

20 


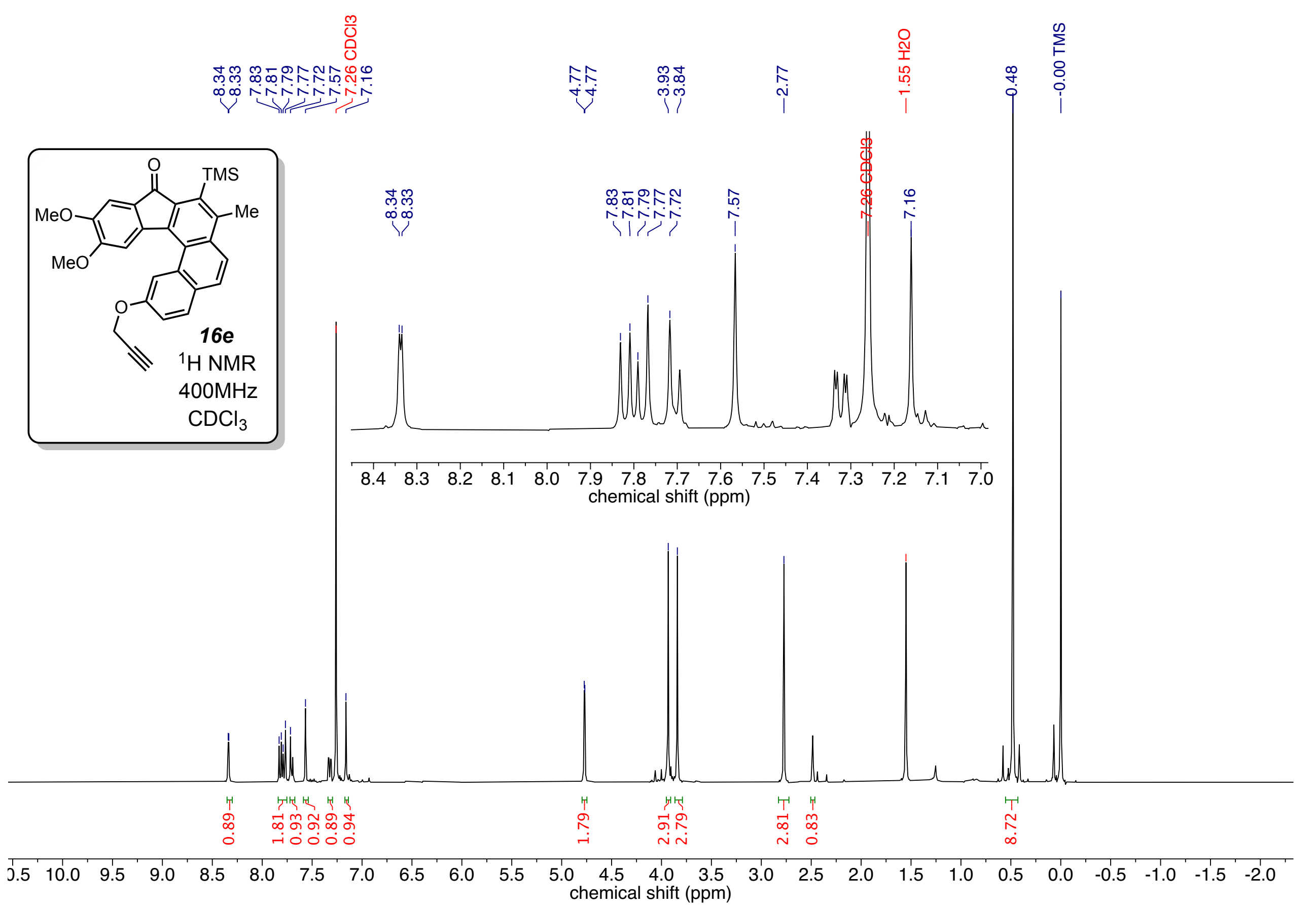



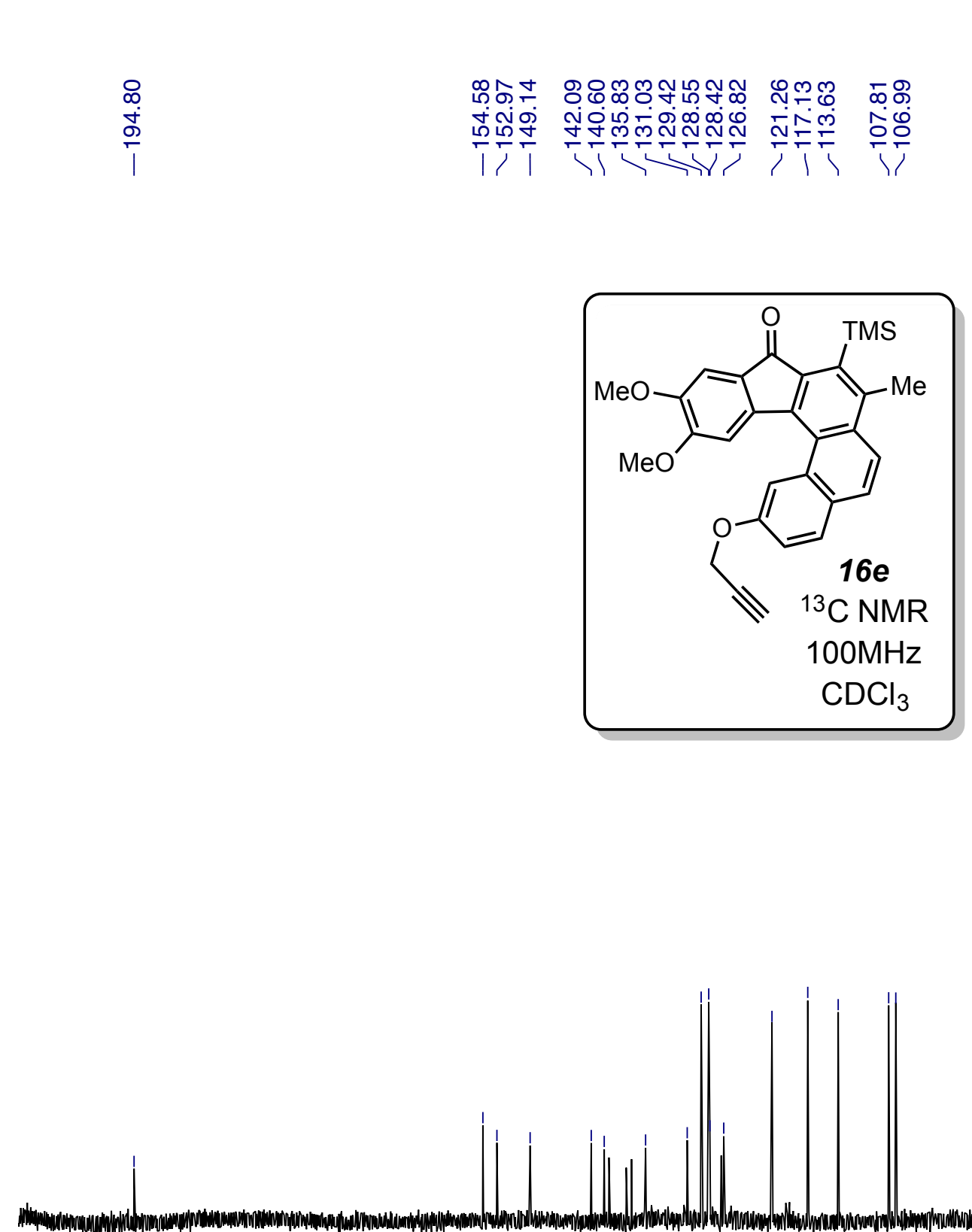

$\begin{array}{lllllllllllllllllllll}200 & 190 & 180 & 170 & 160 & 150 & 140 & 130 & 120 & \begin{array}{l}110 \\ \text { chemical shift (ppm) }\end{array} & 80 & 70 & 60 & 50 & 40 & 30 & 20 & 10 & 0 & -10\end{array}$




\section{$\frac{m}{0}$}

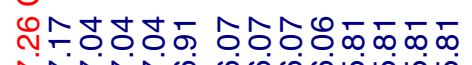

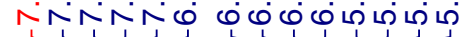

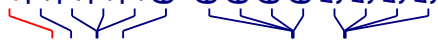
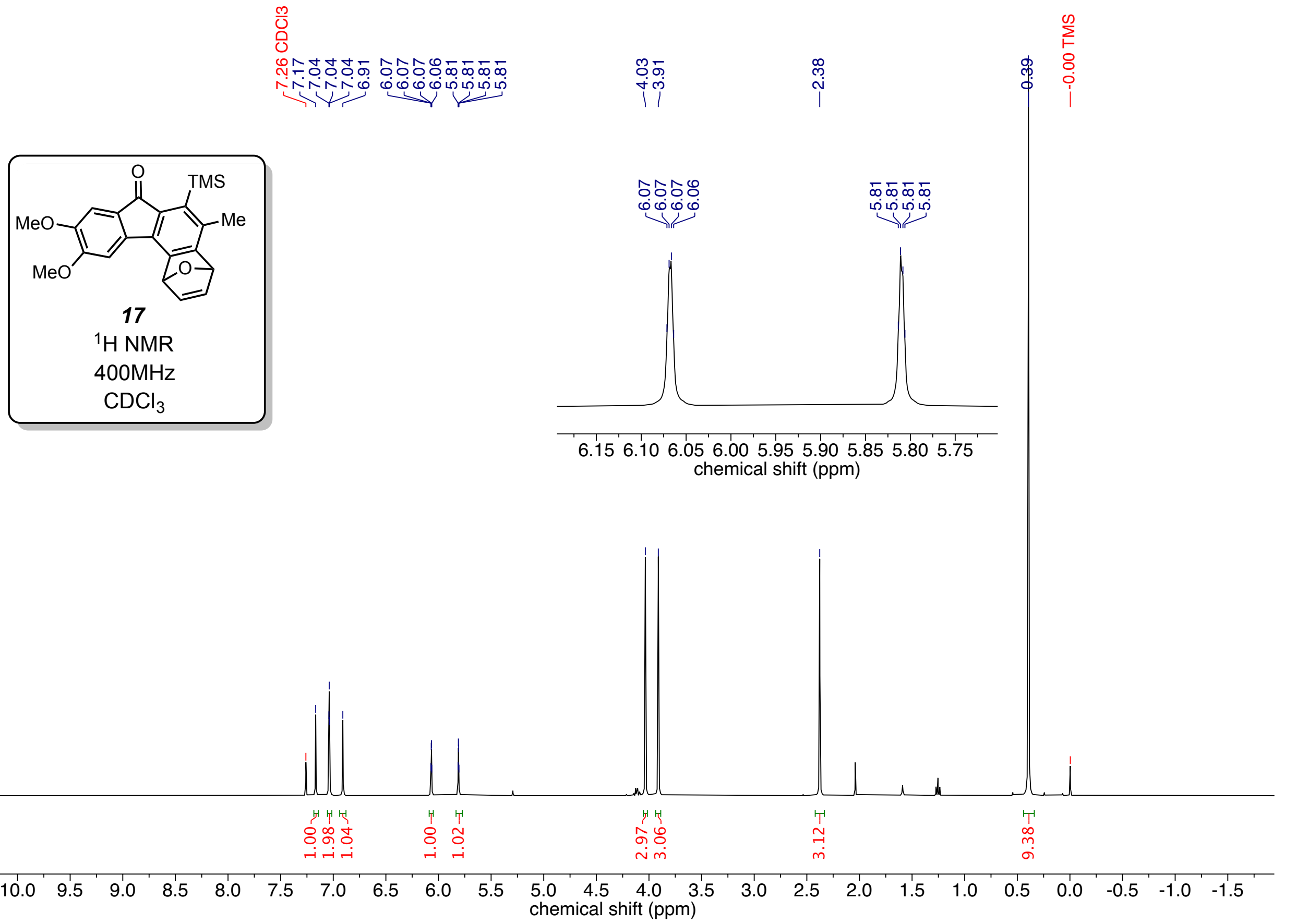

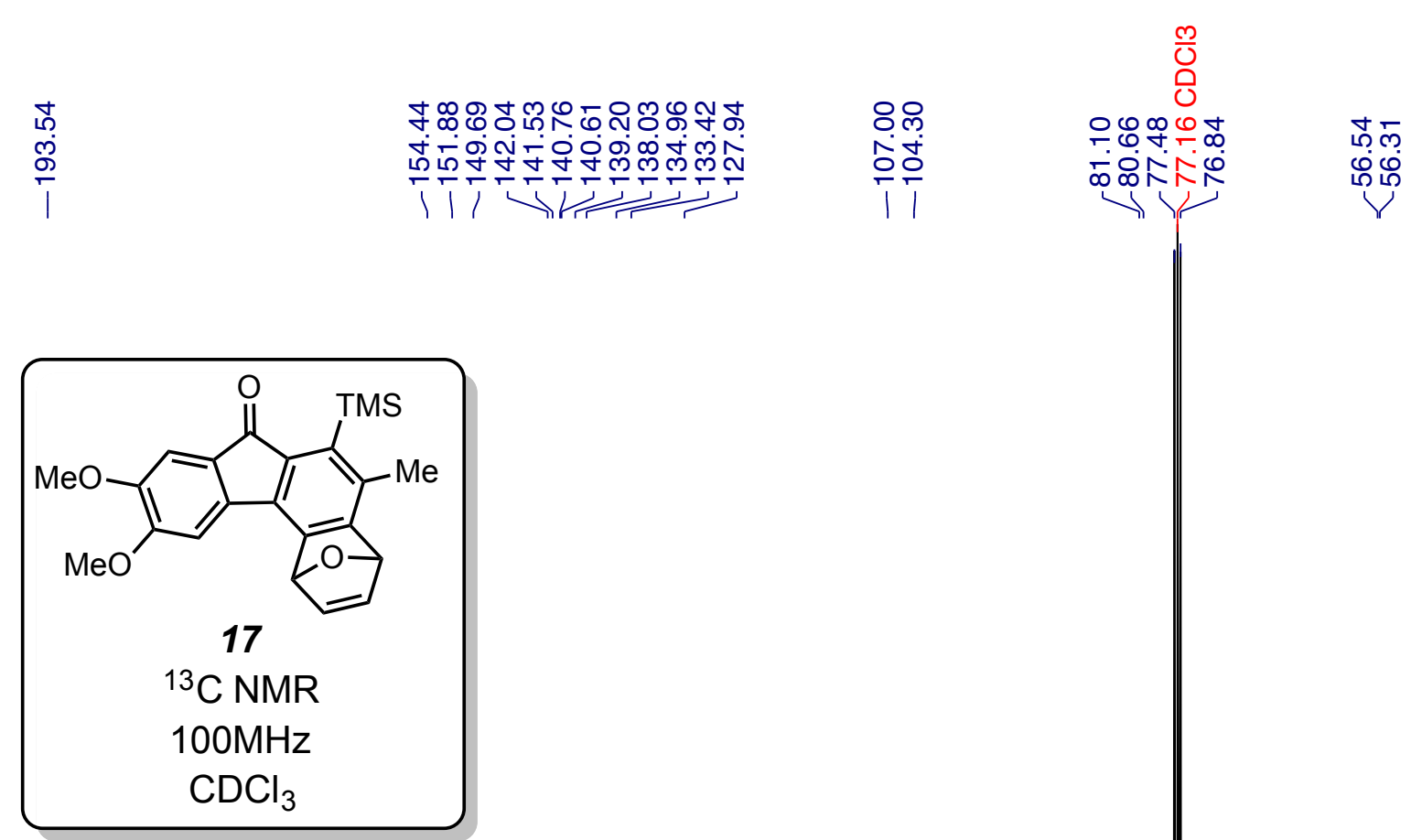

@̊

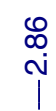

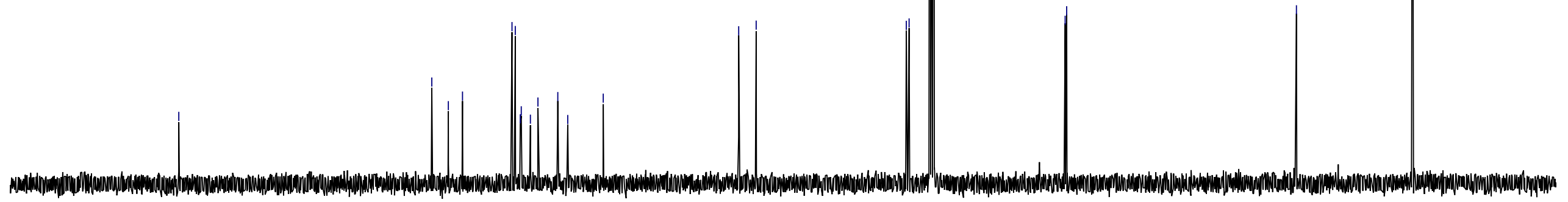

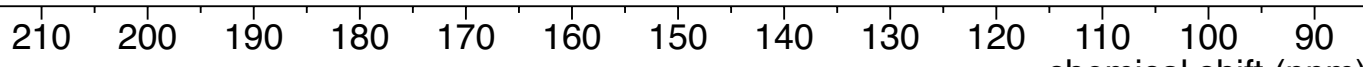
chemical shift (ppm) 


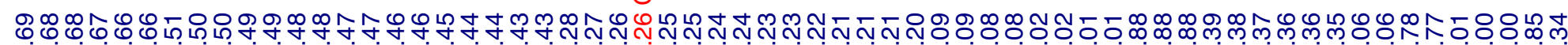

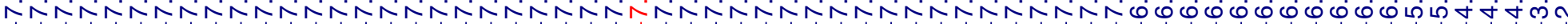
$\frac{m}{0}$

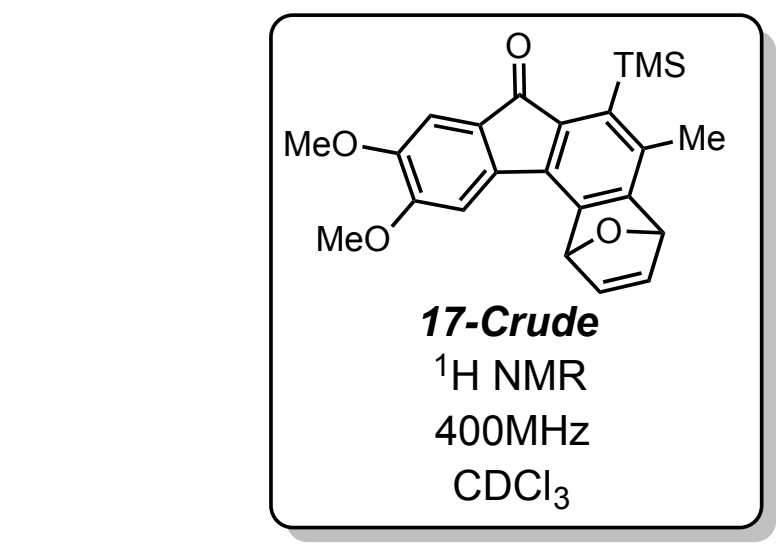

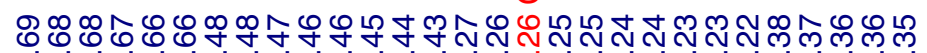

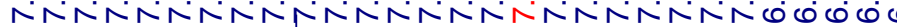

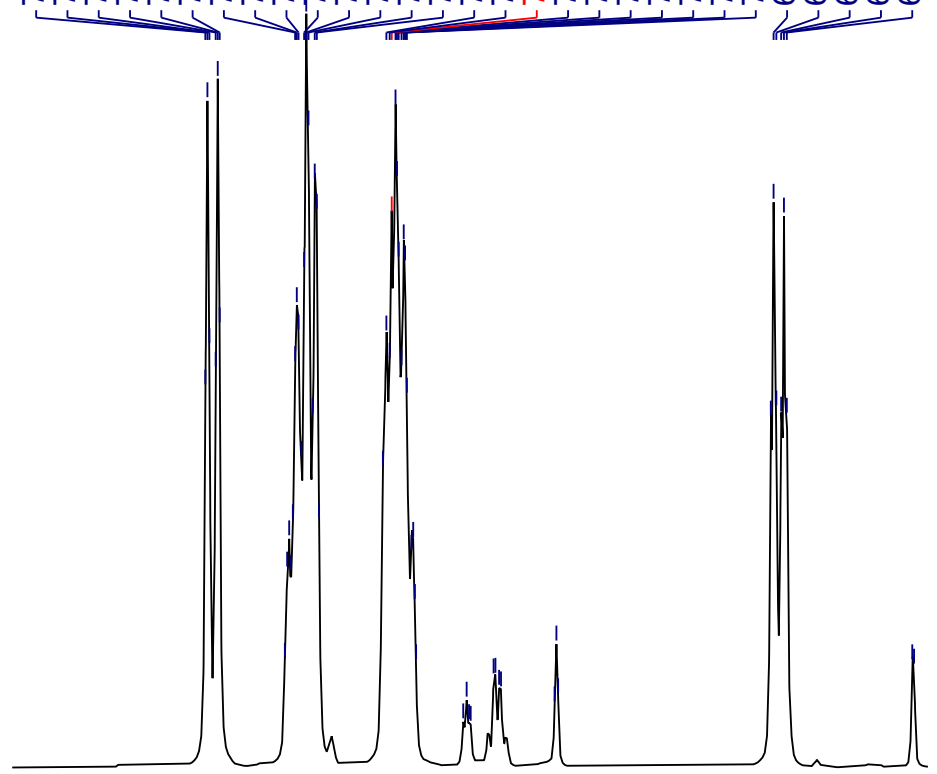

लिळ्ल
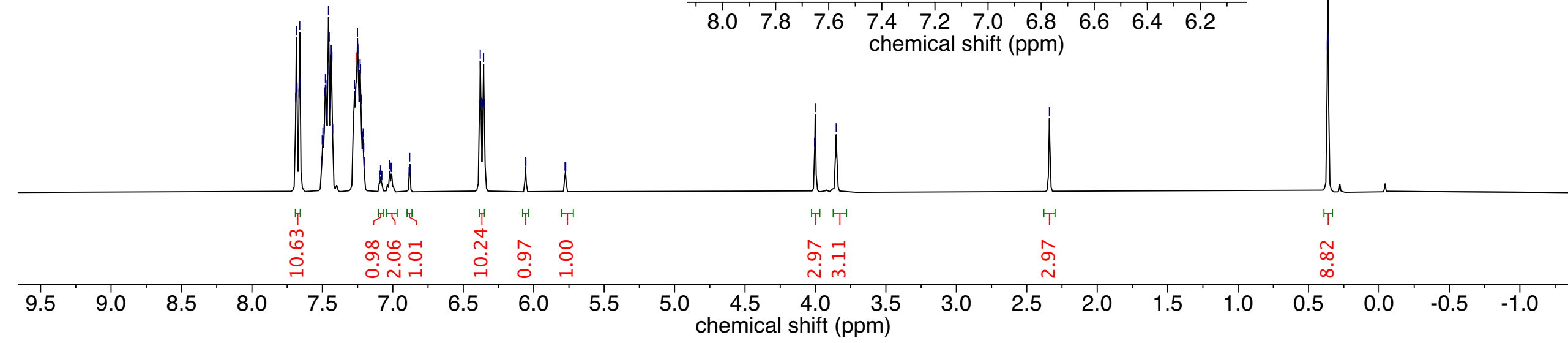


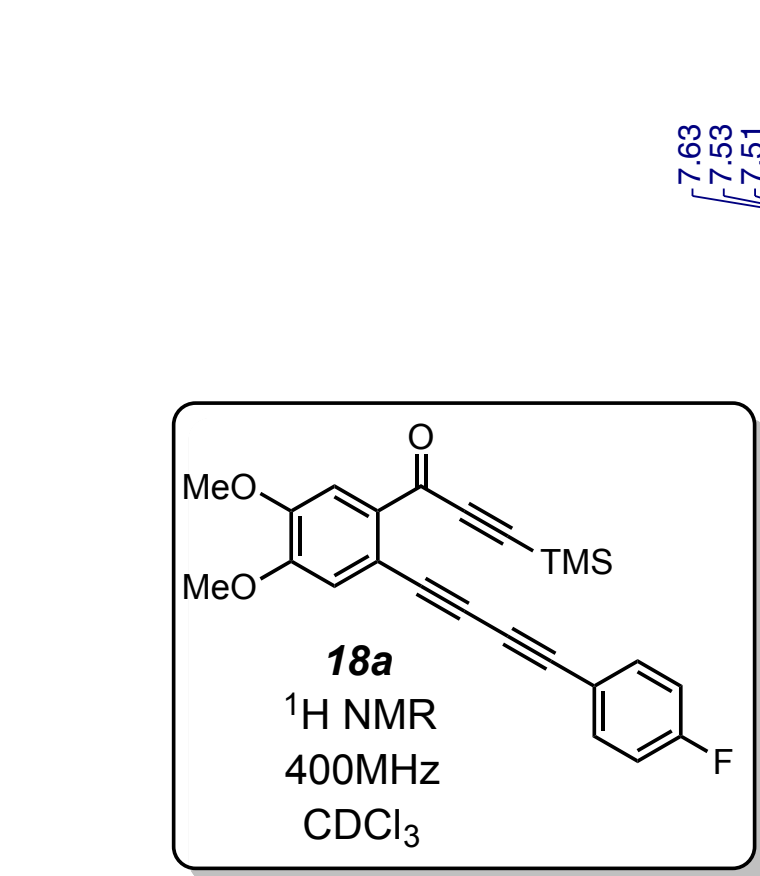

$\frac{m}{0}$

ชณำ

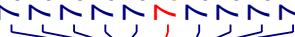

ㄴ.
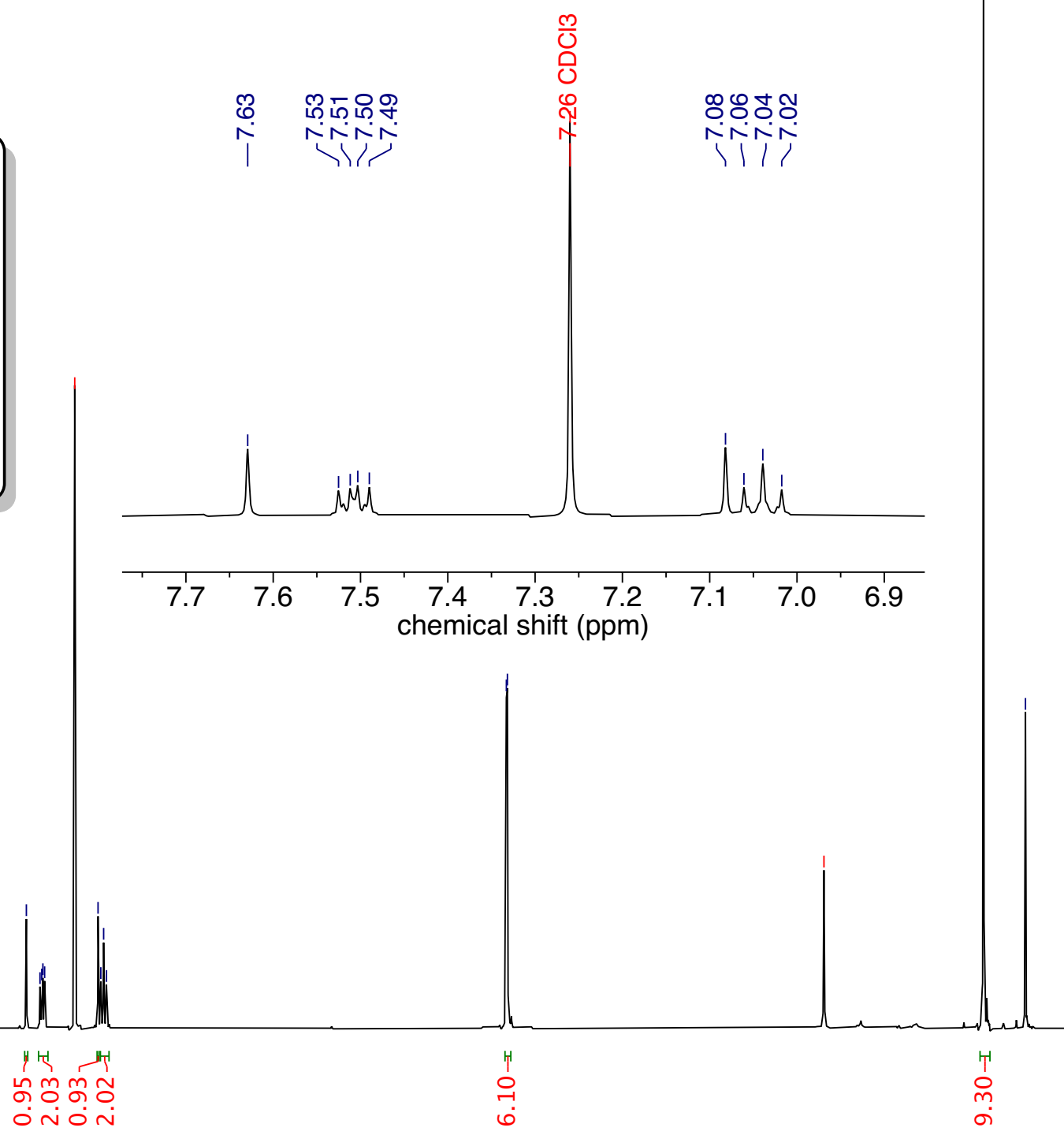

๑ே ฺ

mु

ำ
I
$\stackrel{+}{+}$

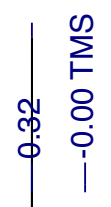

$\begin{array}{lllllllllllllll}12.0 & 11.5 & 11.0 & 10.5 & 10.0 & 9.5 & 9.0 & 8.5 & 8.0 & 7.5 & 7.0 & 6.5\end{array}$

$\begin{array}{lllllll}6.0 & 5.5 & 5.0 & 4.5 & 4.0 & 3.5\end{array}$ chemical shift (ppm) 

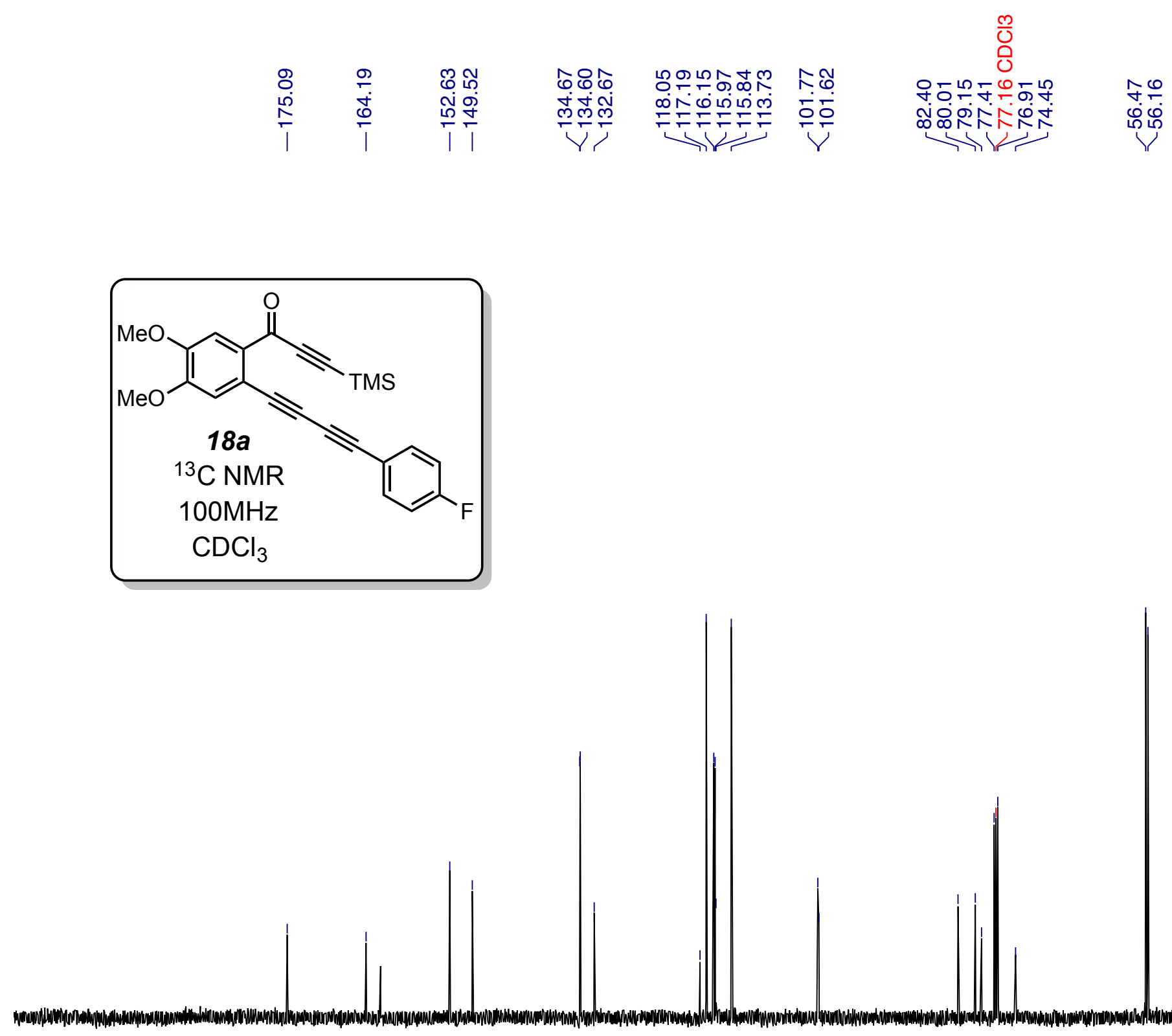

\begin{tabular}{llllllllllllllllllllllll}
\hline 210 & 200 & 190 & 180 & 170 & 160 & 150 & 140 & 130 & 120 & $\begin{array}{c}110 \\
\text { chemical shift (ppm) }\end{array}$ & 80 & 70 & 60 & 50 & 40 & 30 & 20 & 10 & 0 & -10
\end{tabular}




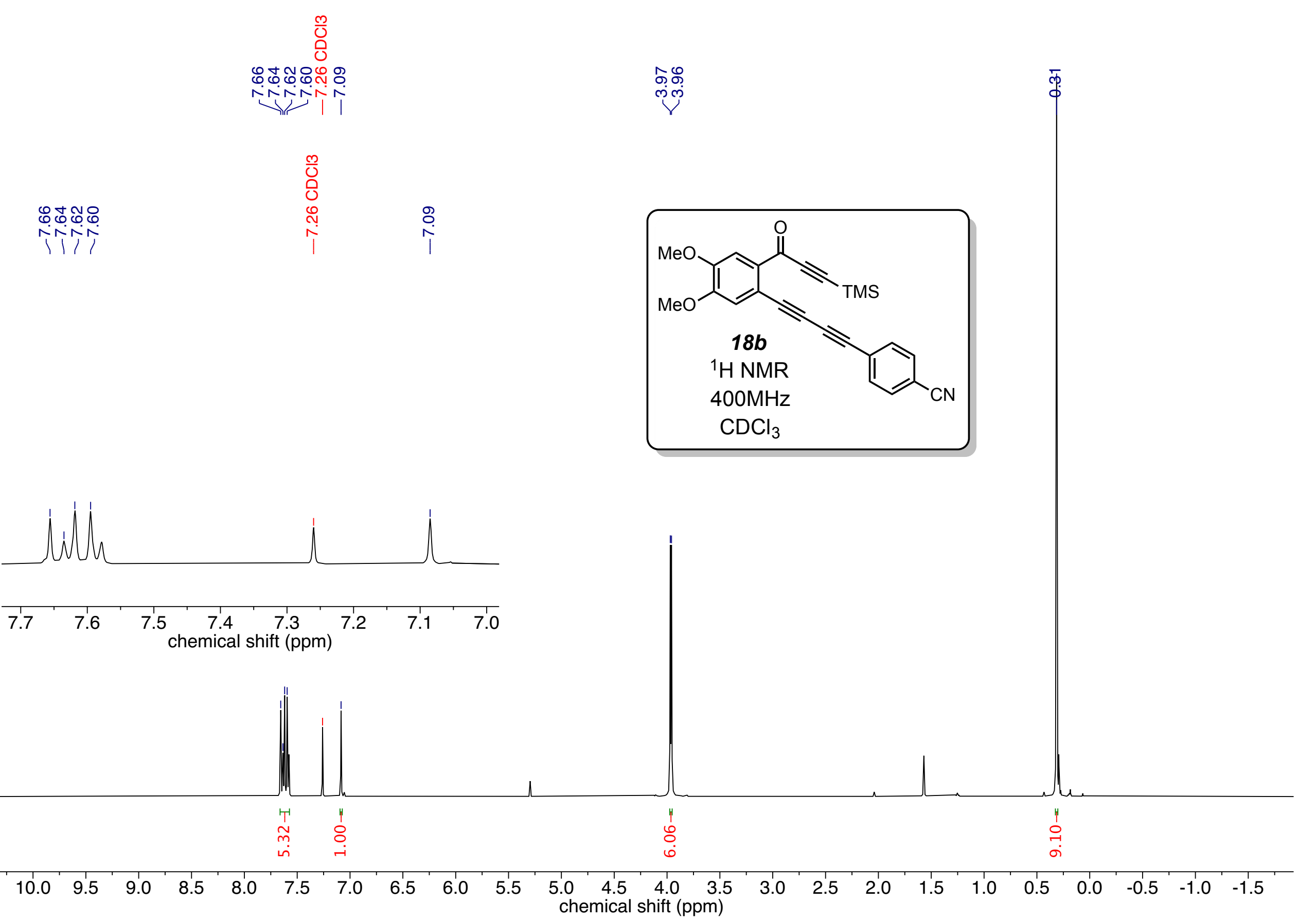



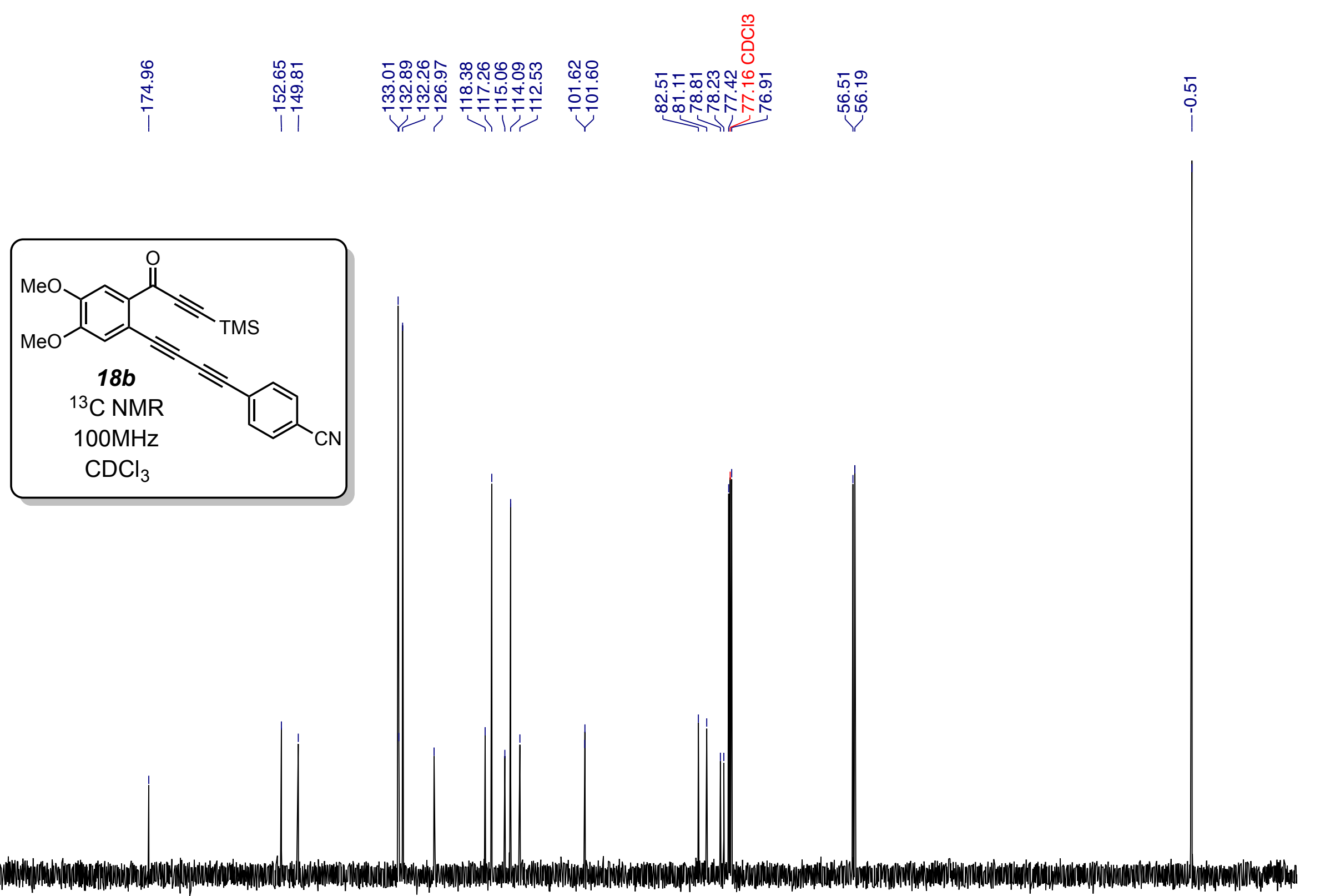

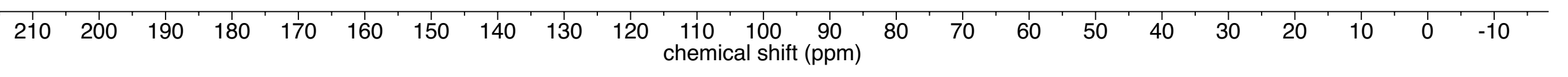



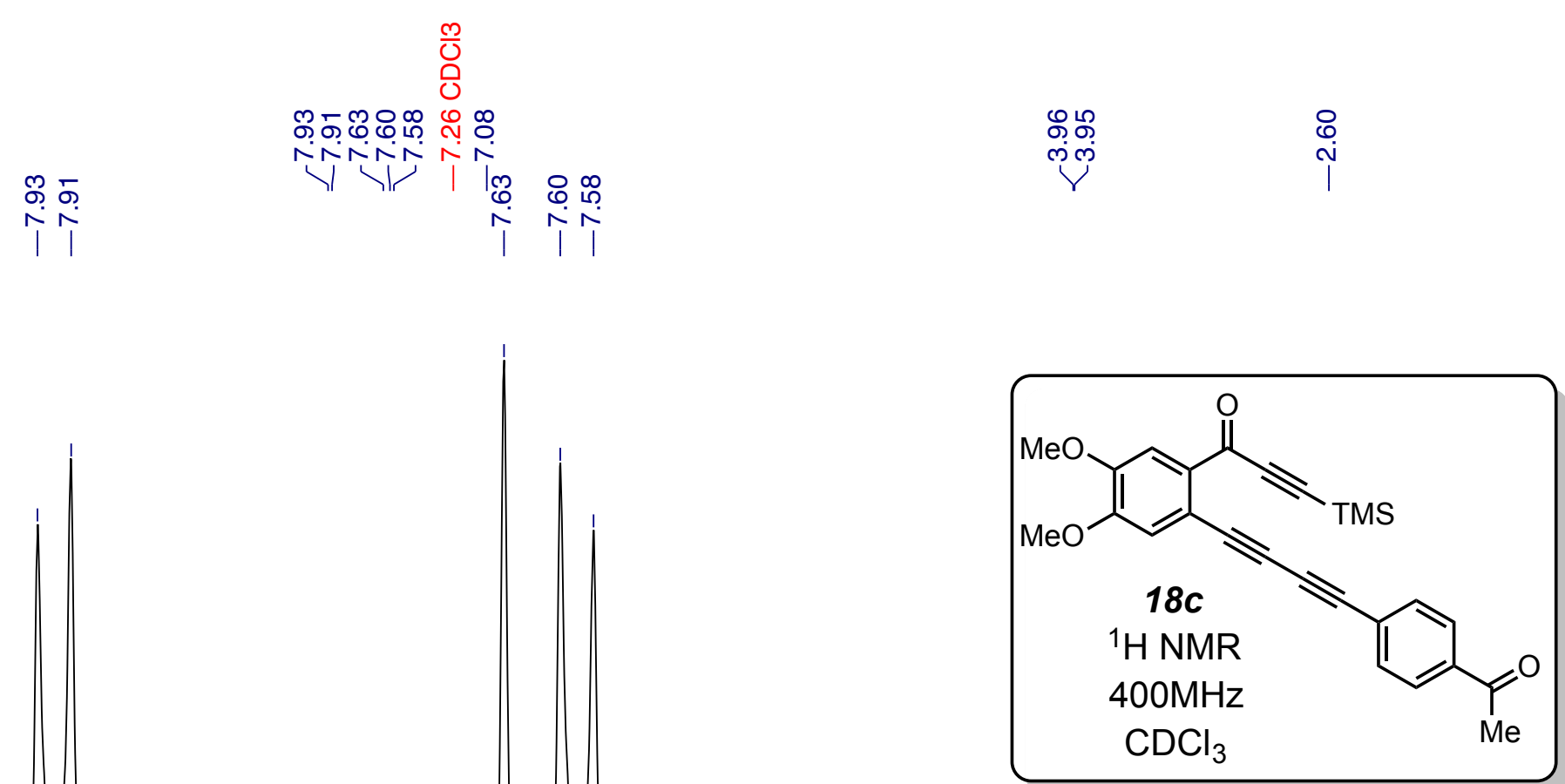

\begin{tabular}{llllllllllllllll}
\hline 3.00 & 7.95 & 7.90 & 7.85 & 7.80 & 7.75 & 7.70 & 7.65 & 7.60 & 7.55 & 7.50
\end{tabular} chemical shift (ppm)

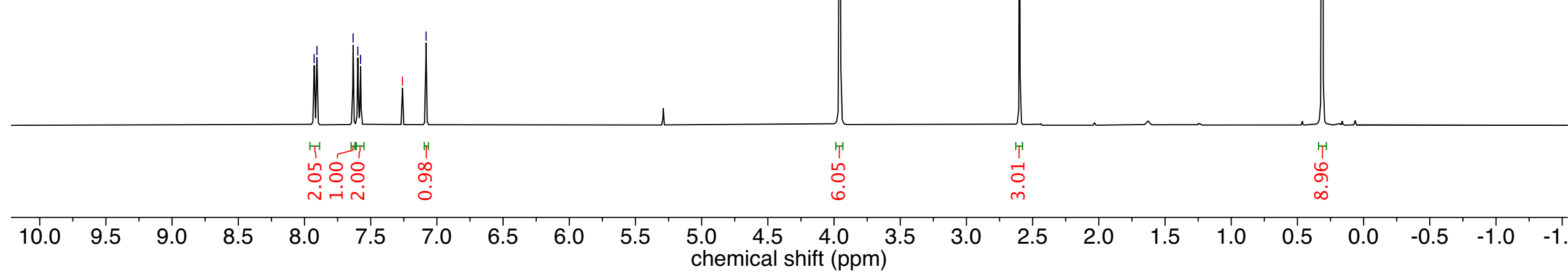




\begin{tabular}{|c|c|c|c|c|c|c|}
\hline $\begin{array}{l}\bar{N} \\
\stackrel{\sigma}{\sigma} \\
\end{array}$ & $\begin{array}{l}8 \\
\stackrel{i}{\Gamma} \\
\frac{1}{1}\end{array}$ & 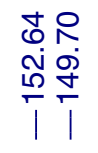 & 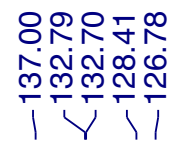 & 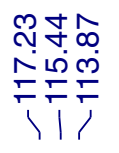 & 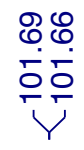 & 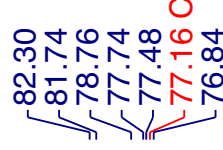 \\
\hline
\end{tabular}
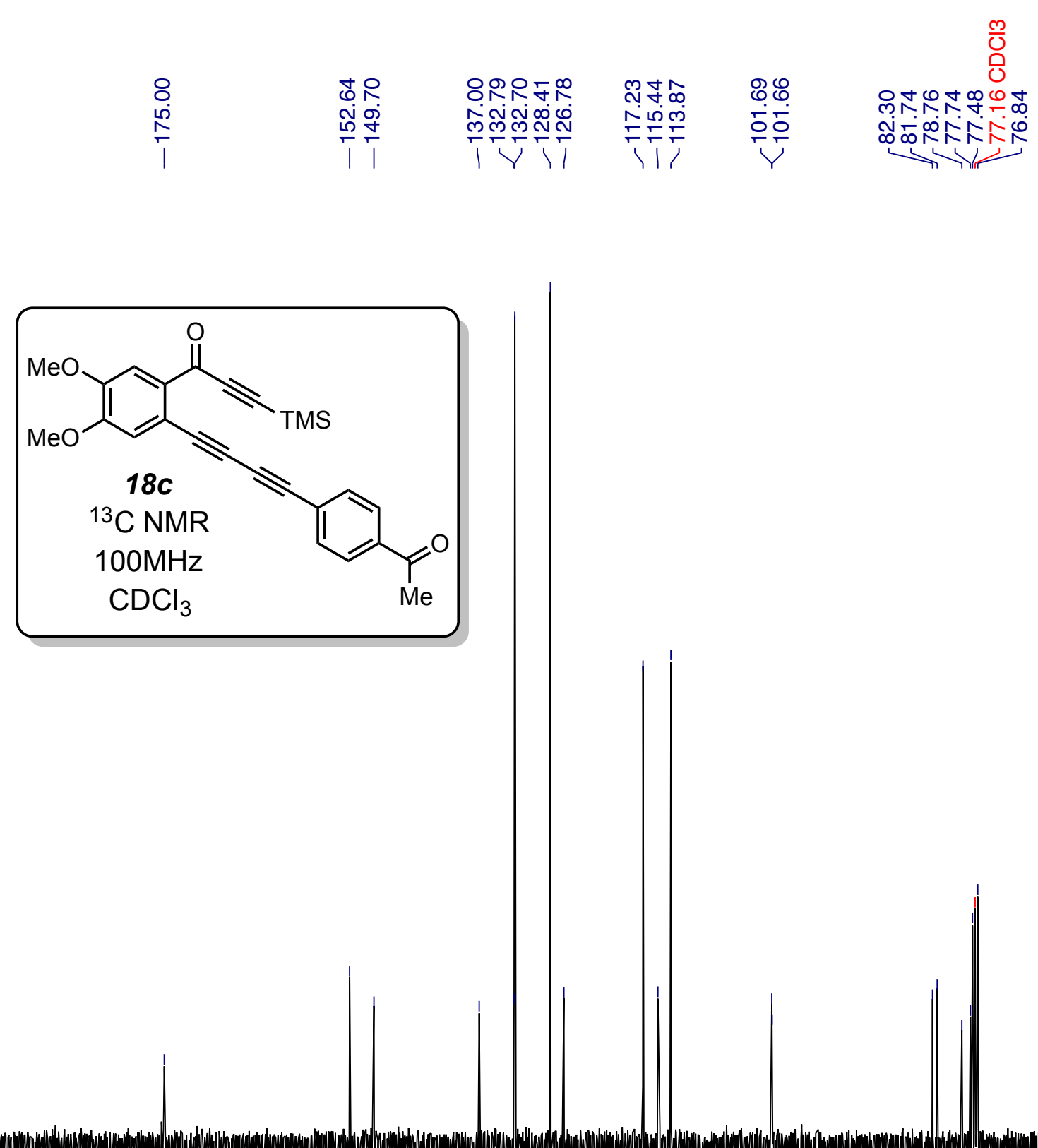

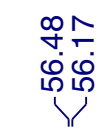

$\stackrel{N}{\stackrel{N}{\grave{i}}}$

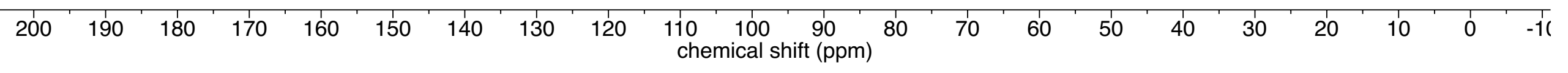




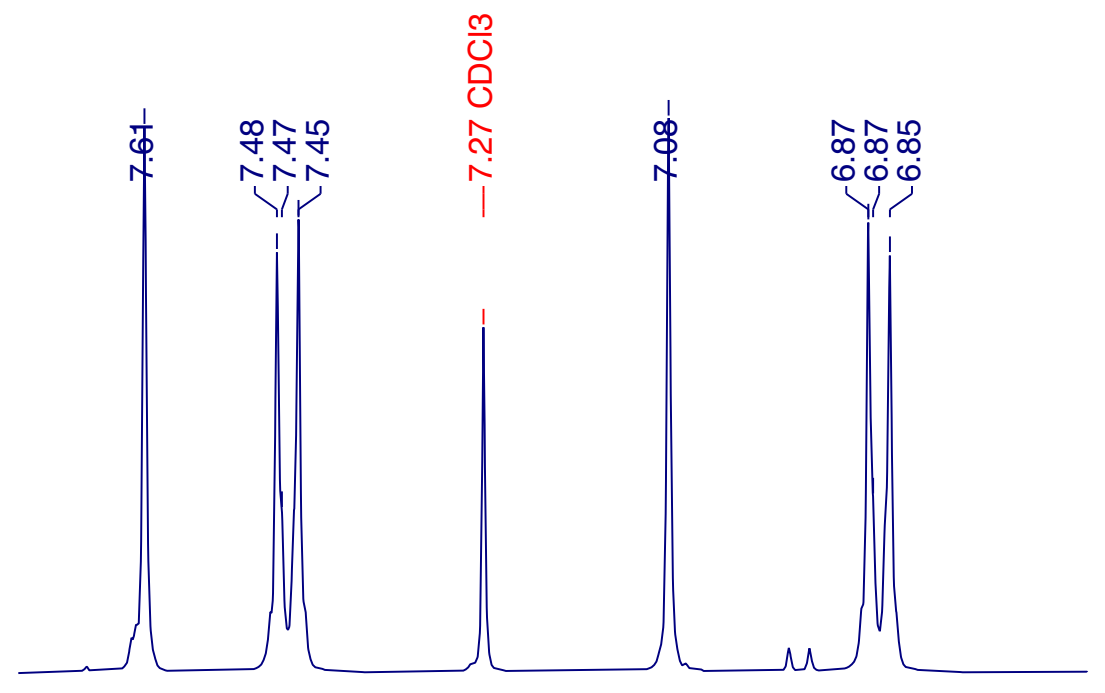

ले
ம்

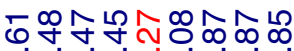

ÑÑ̃No்

(1) लुलm

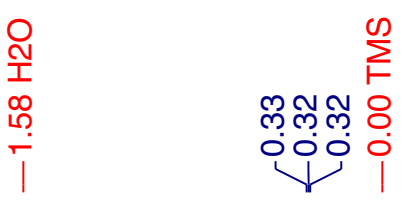

$\sum_{1}^{n}$
$\infty$

\begin{tabular}{lllllllllll}
\hline 7.7 & 7.6 & 7.5 & 7.4 & 7.3 & 7.2 & 7.1 & 7.0 & 6.9 & 6.8 & 6.7 \\
& & & & & \\
chemical shift $(\mathrm{ppm})$ & & & &
\end{tabular}

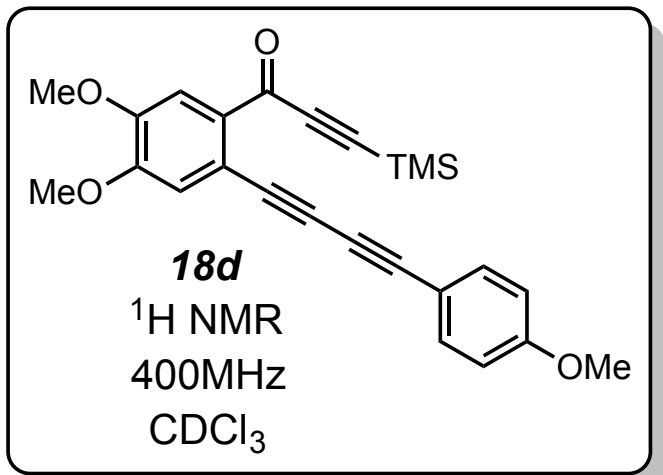

i.

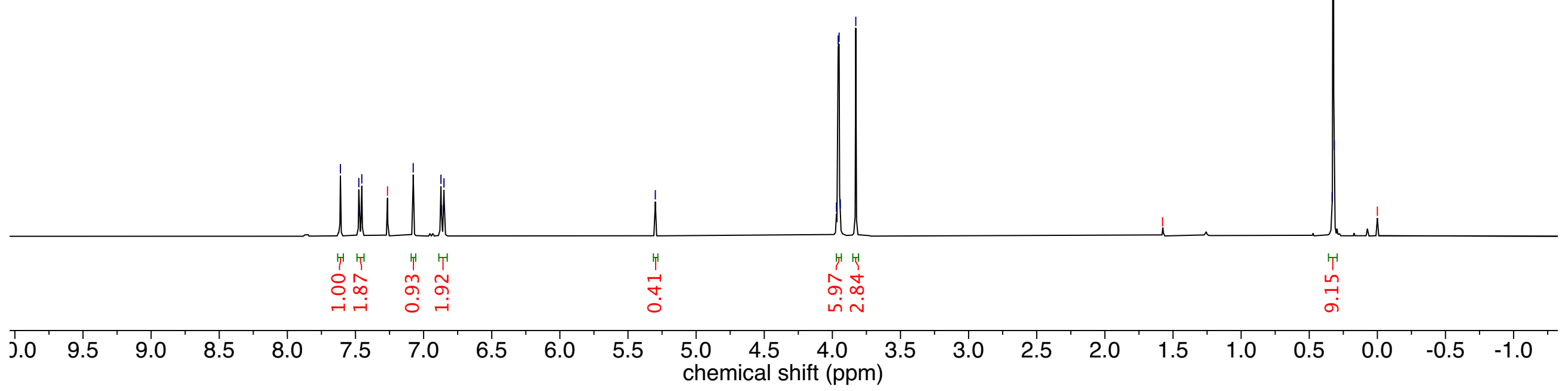




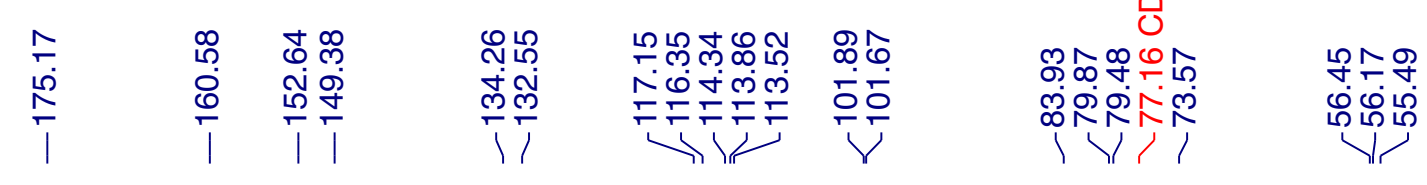

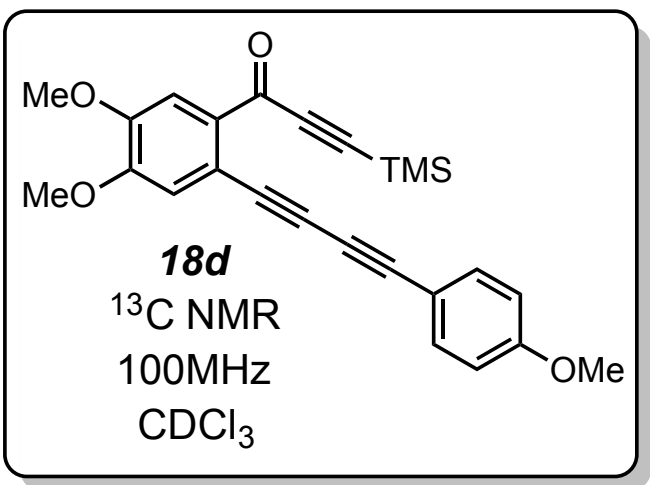

\|\|$\|_{i}$

21

$210 \quad 200$

190

180

170

$160 \quad 150$
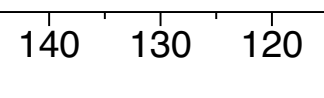

$\begin{array}{ccc}110 & 100 & 90 \\ \text { chemical shift }(\mathrm{ppm})\end{array}$

$\stackrel{\infty}{\stackrel{\infty}{+}}$

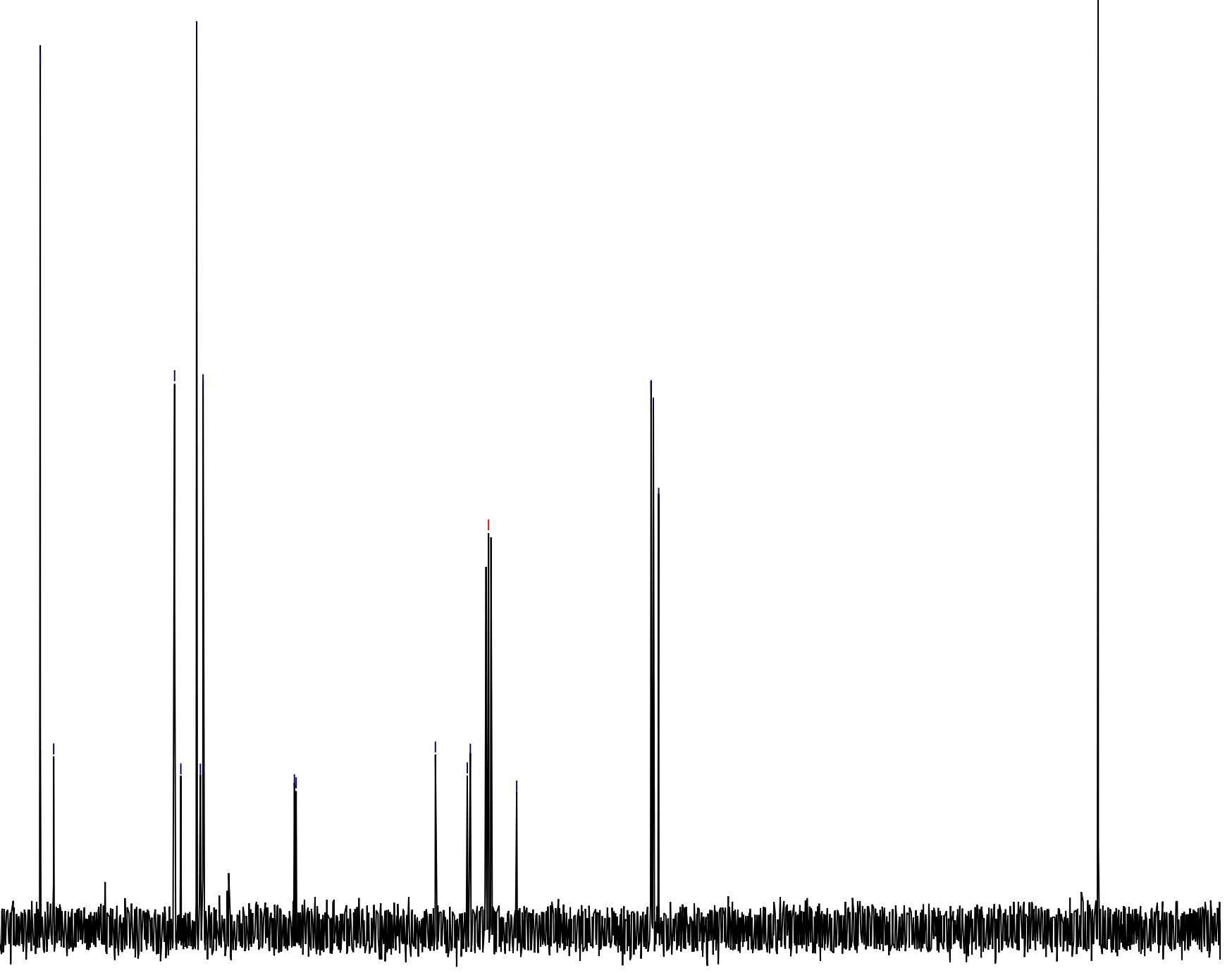



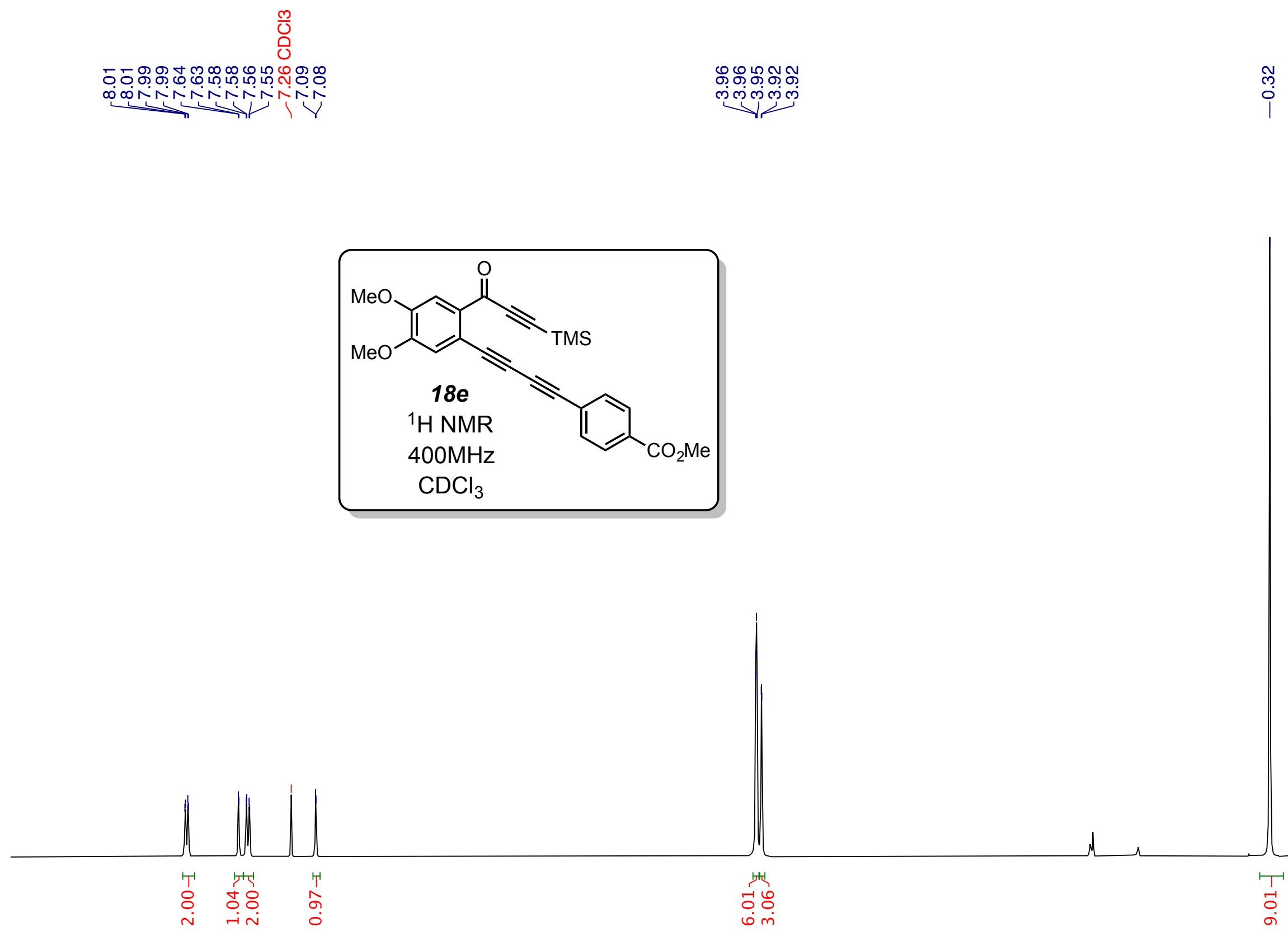

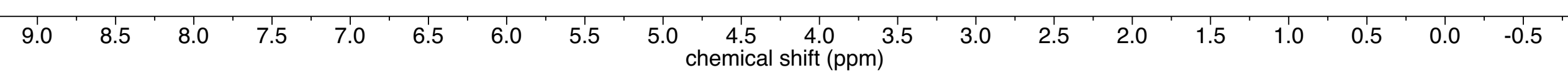




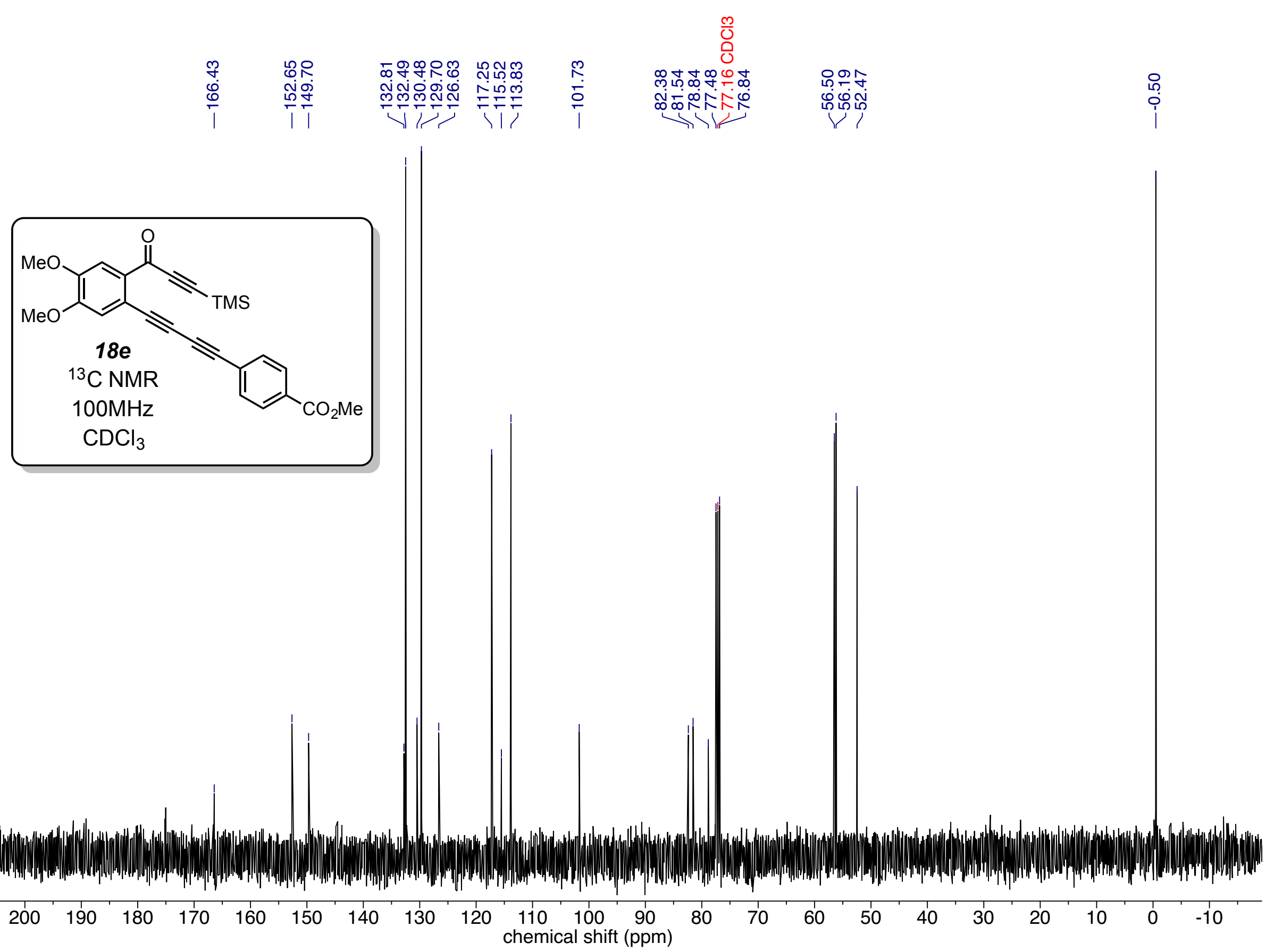




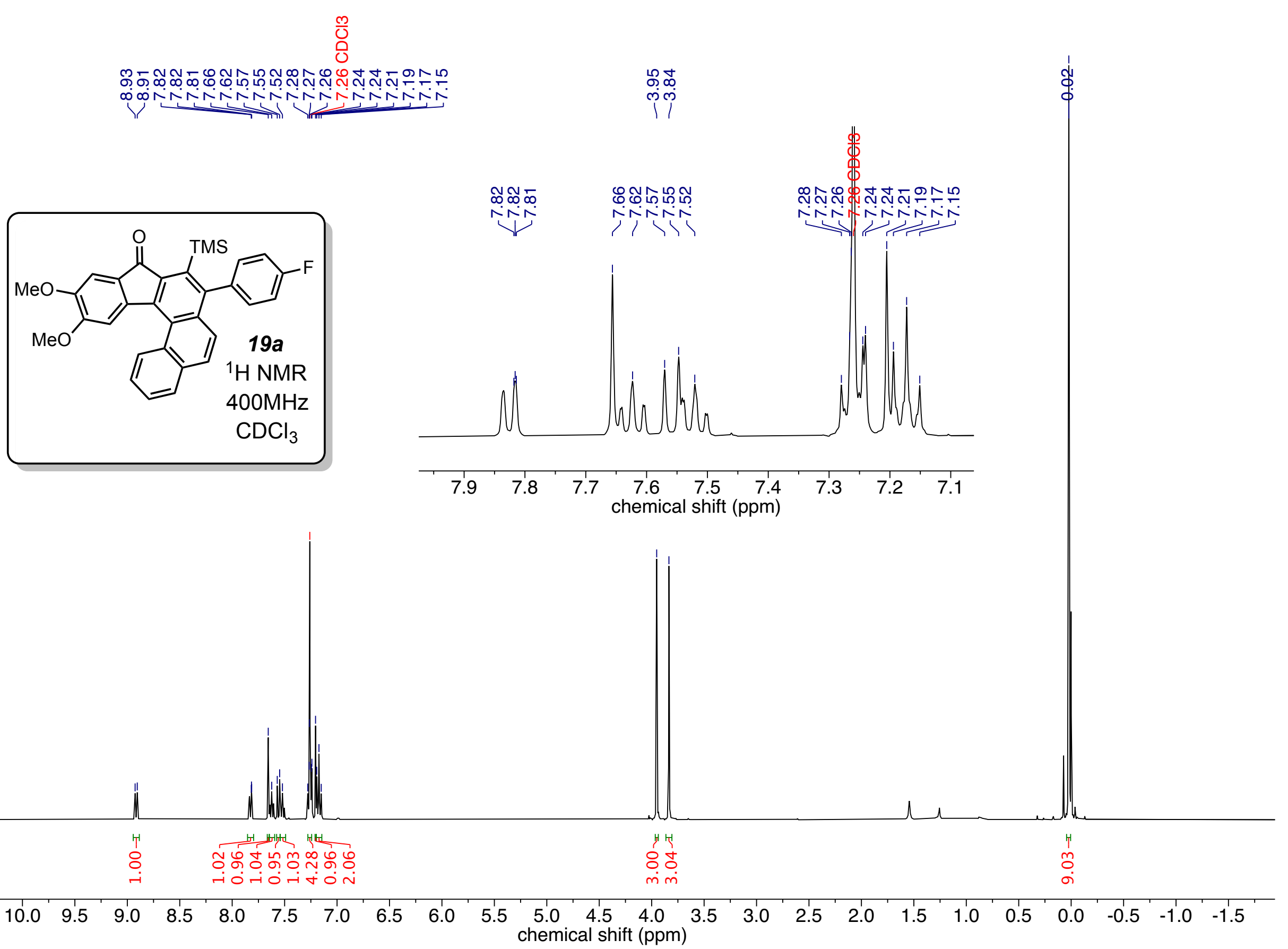



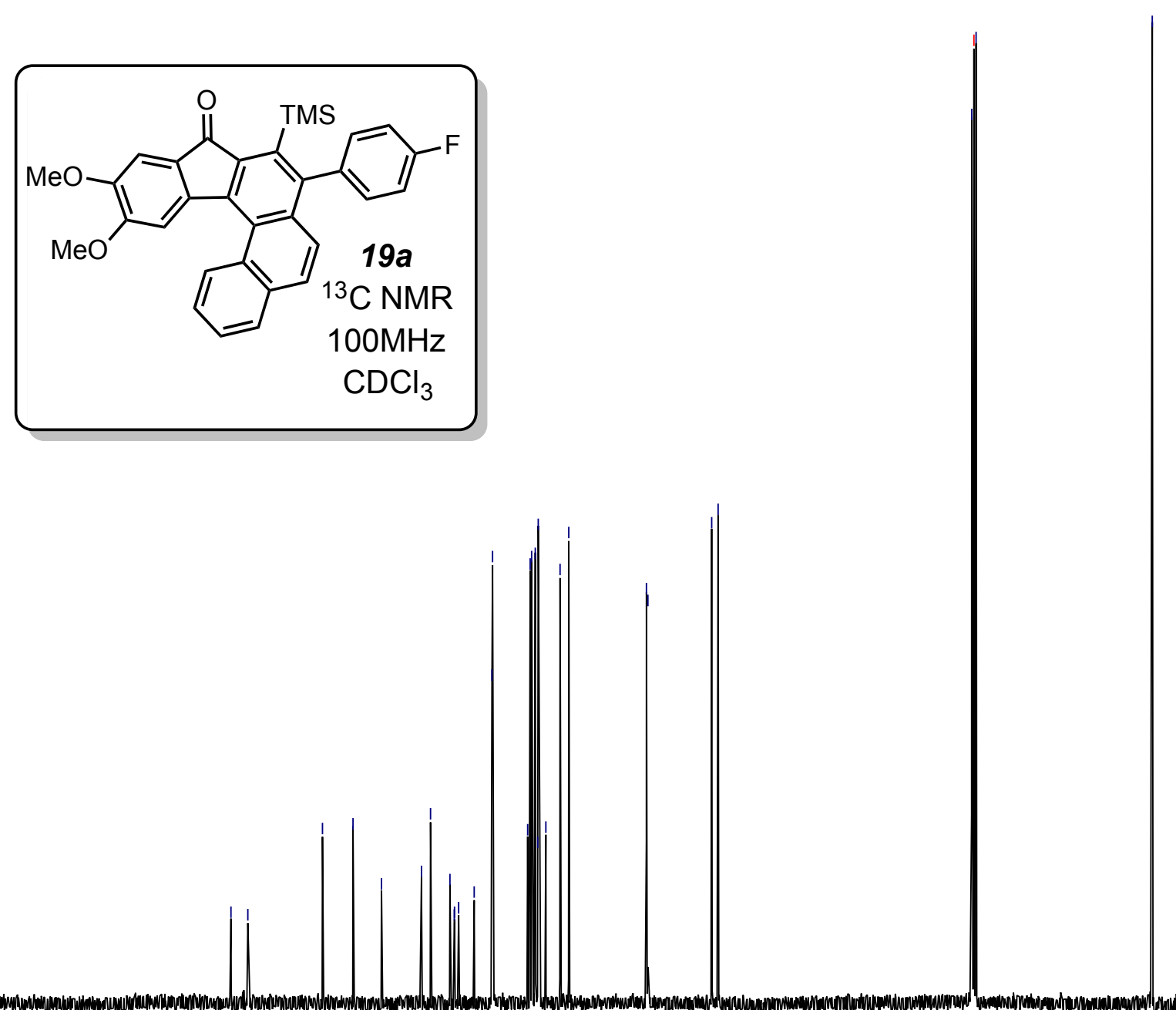

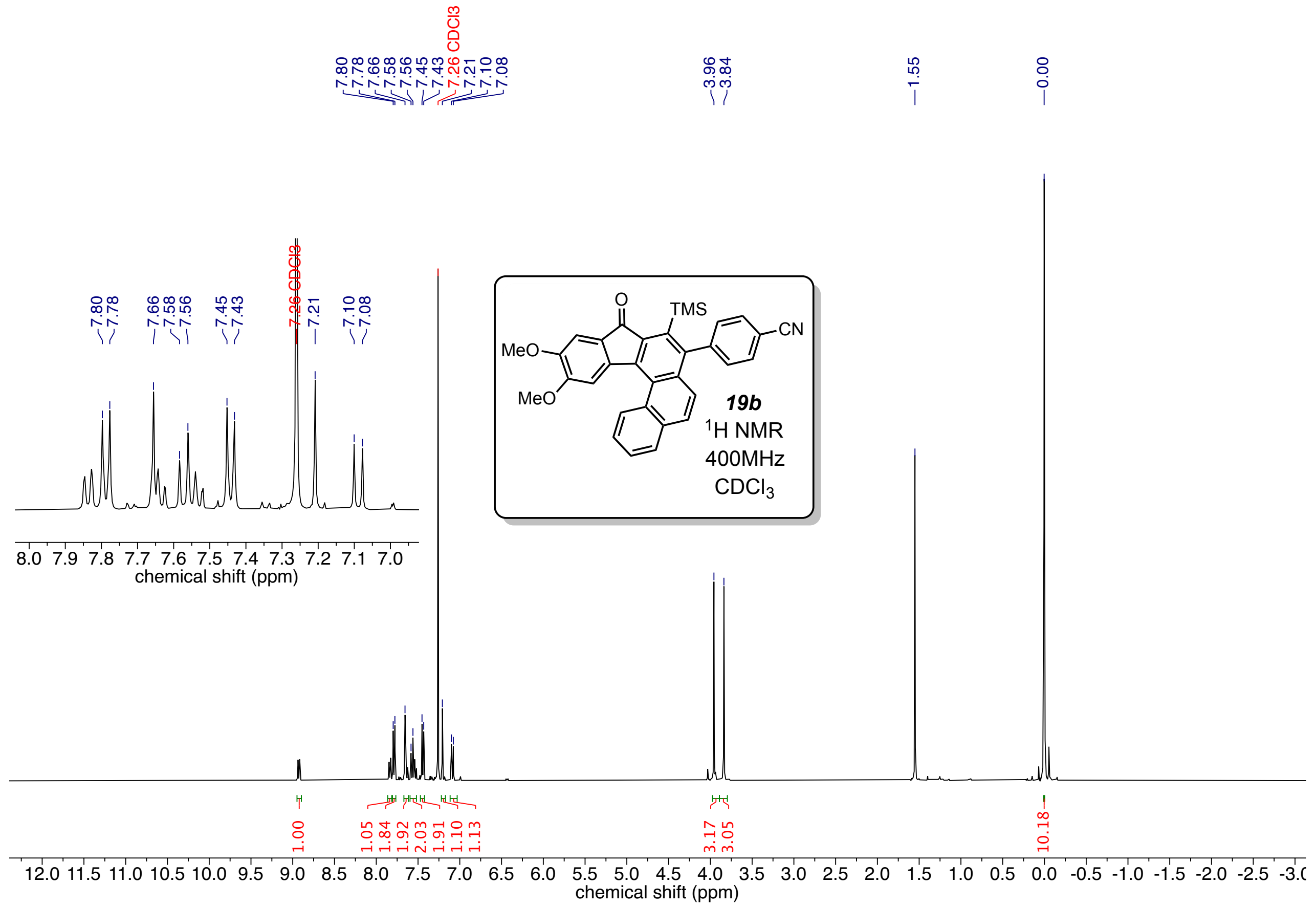

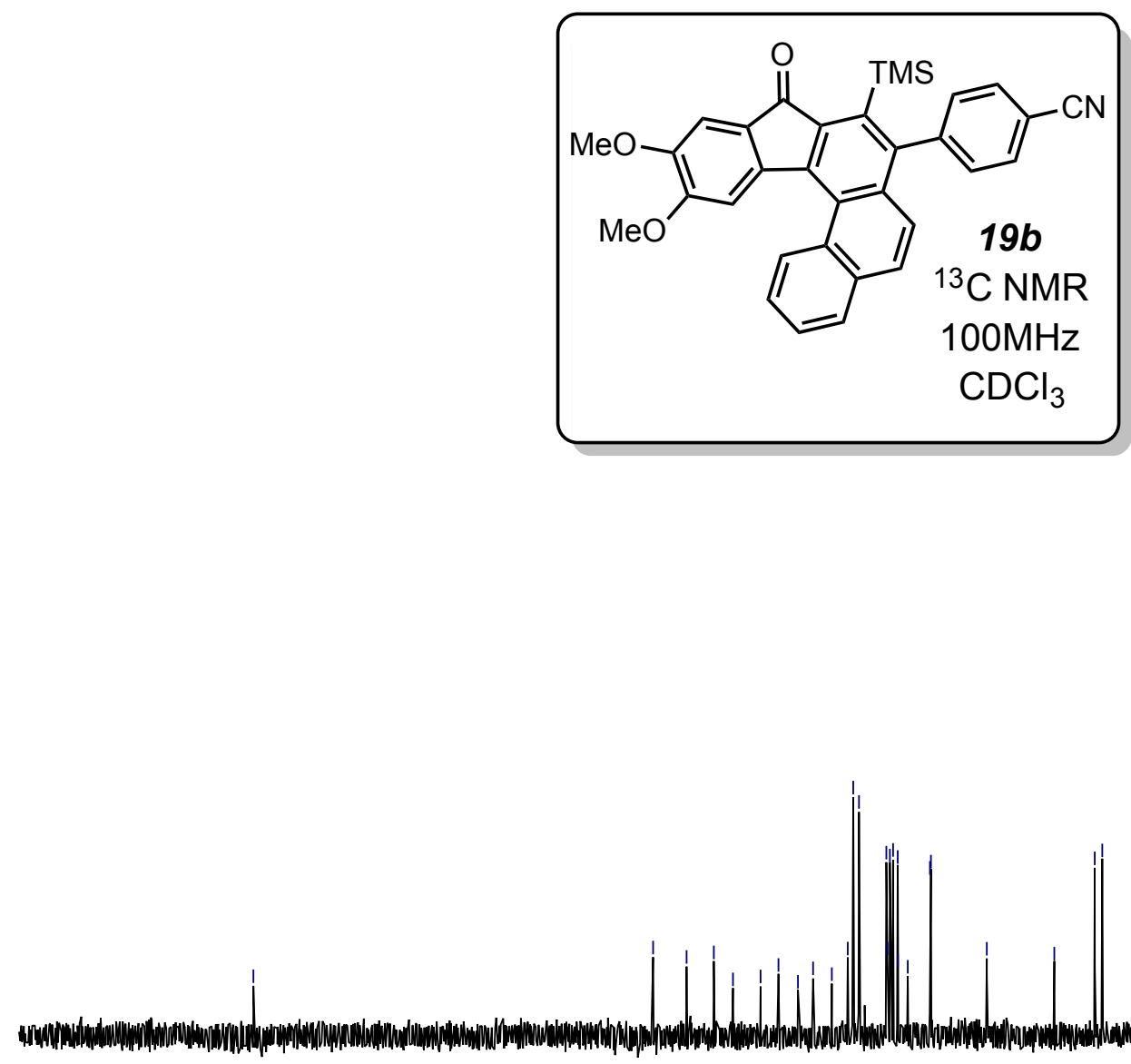

\begin{tabular}{lllllllllllllllllllll}
\hline 210 & 200 & 190 & 180 & 170 & 160 & 150 & 140 & 130 & 120 & $\begin{array}{c}110 \\
\text { chemical shift (ppm) }\end{array}$ & 70 & 60 & 50 & 40 & 30 & 20 & 10 & 0 & -10 & 10
\end{tabular}




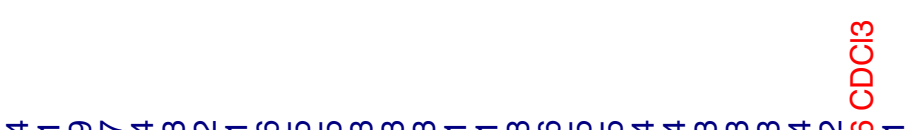

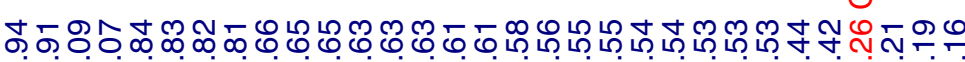

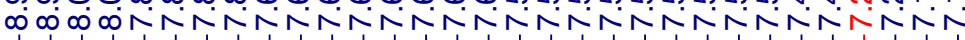
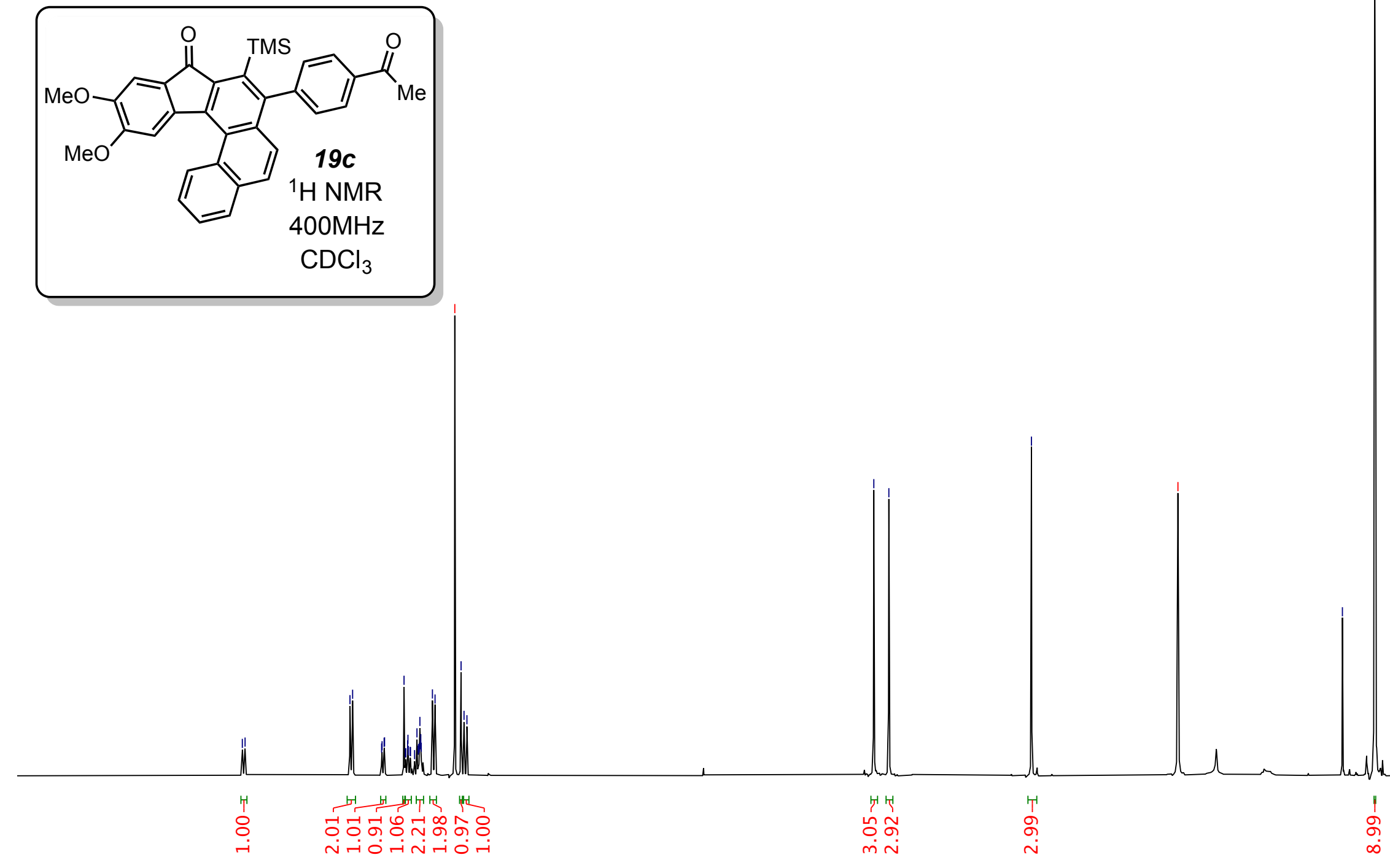

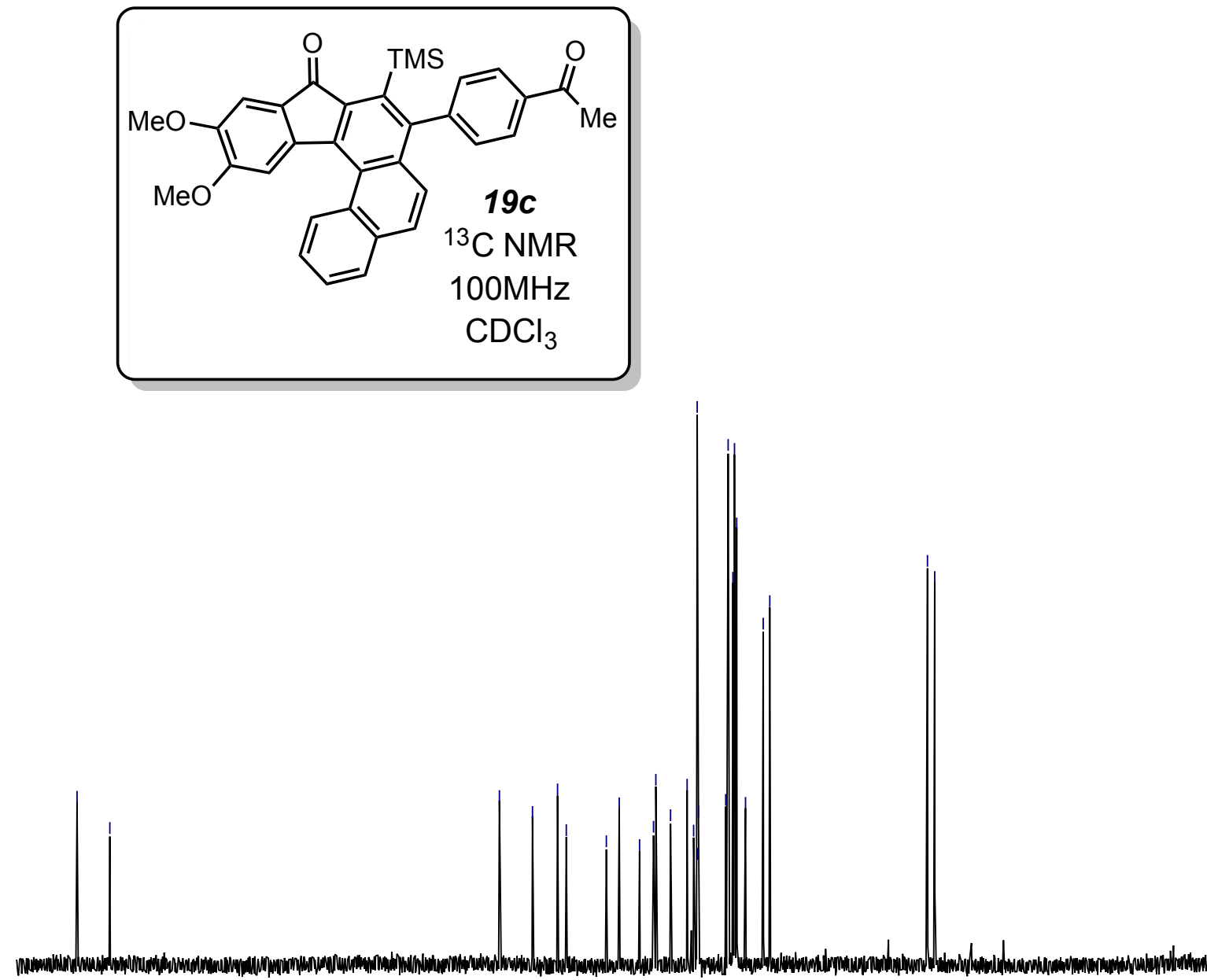

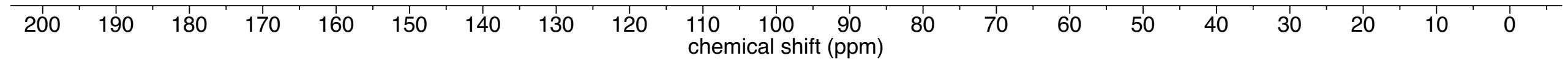




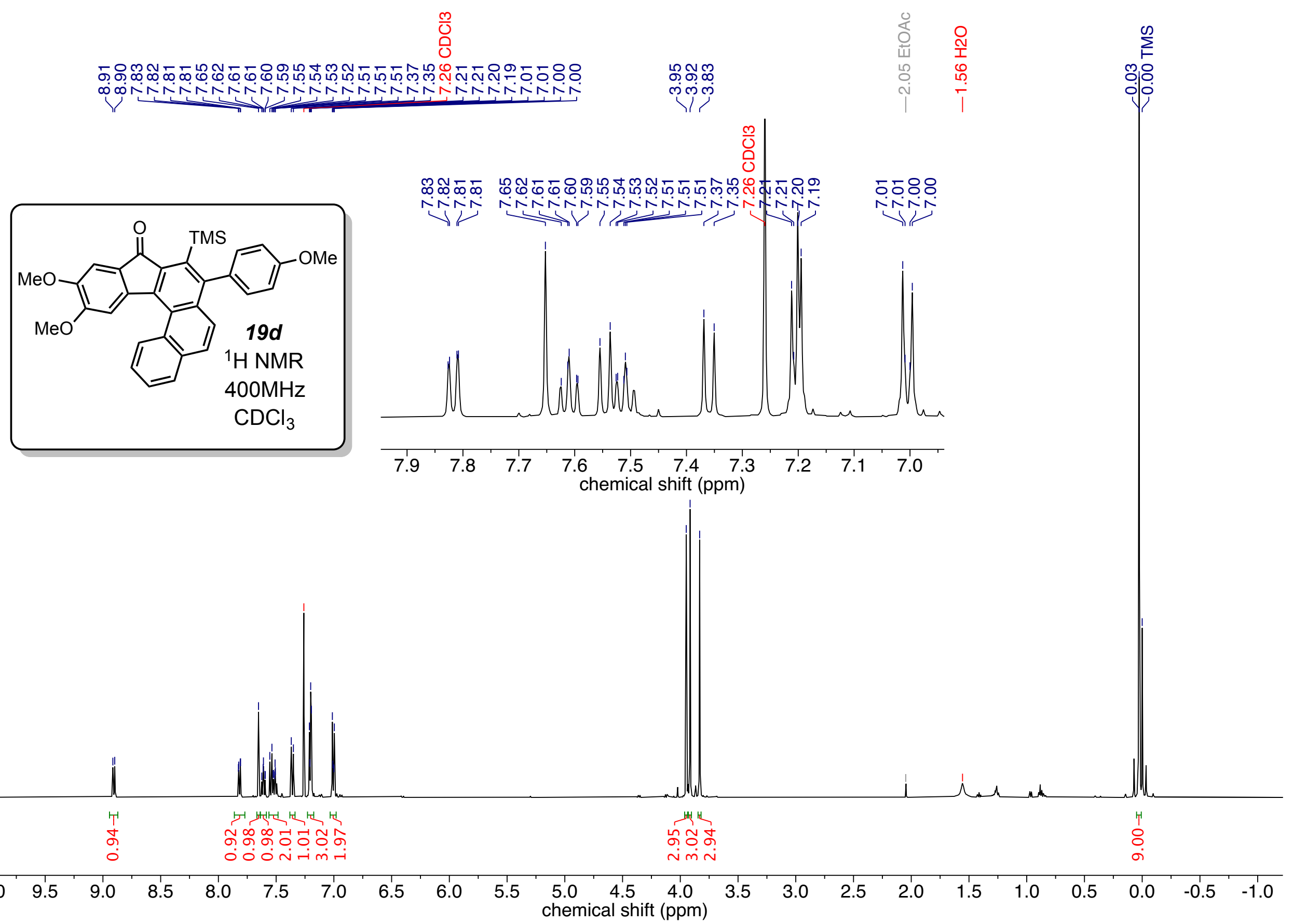



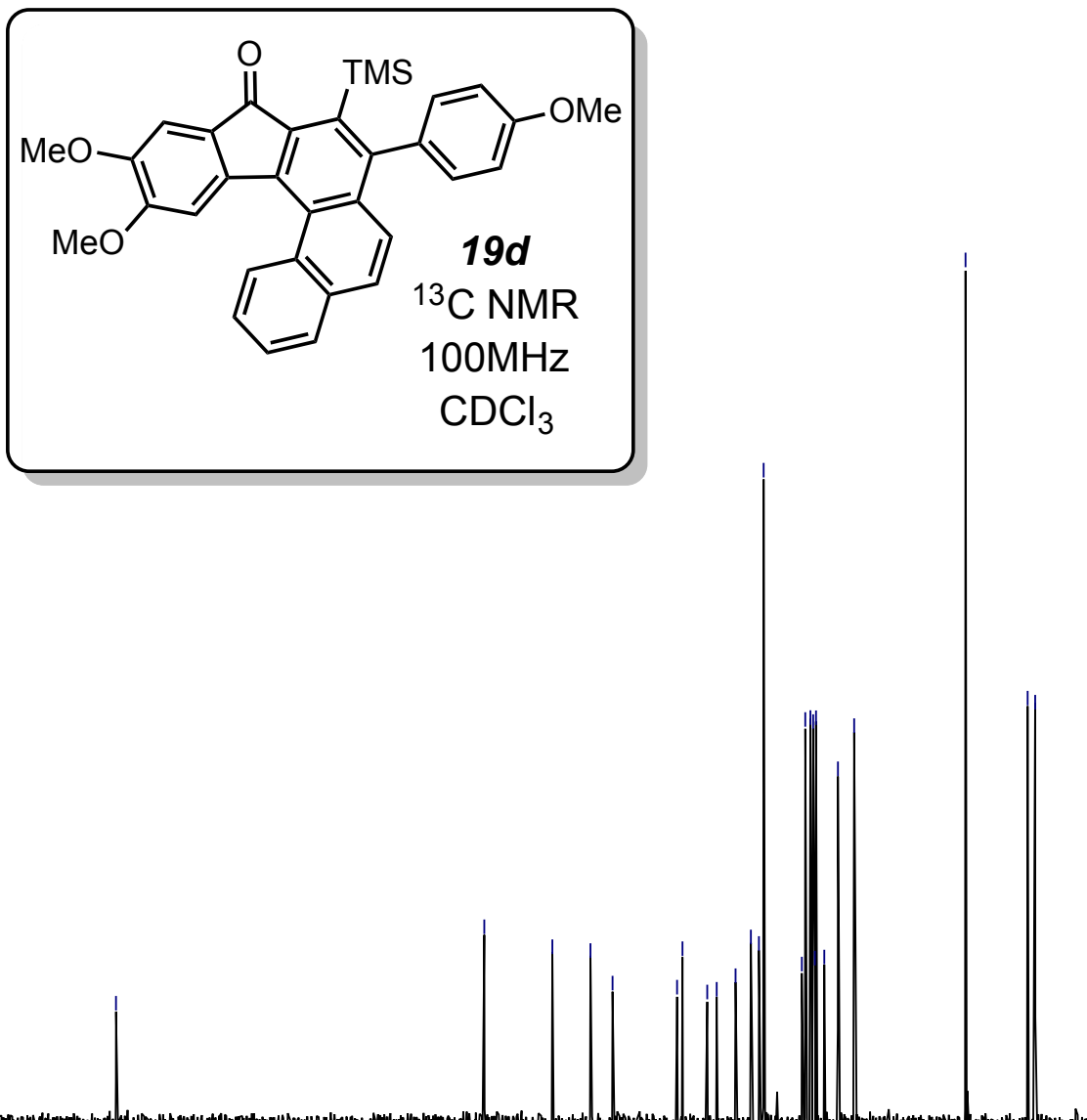


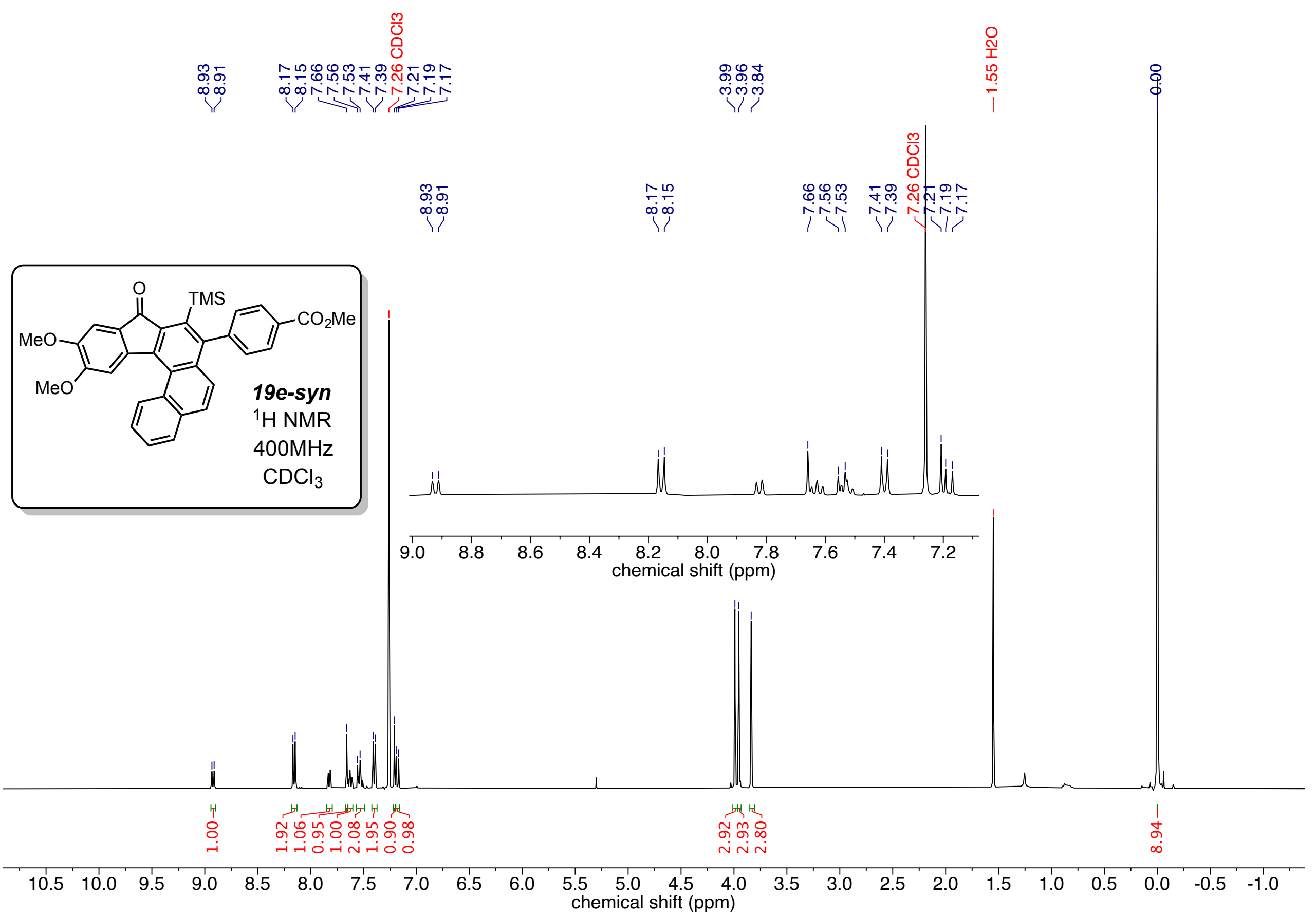



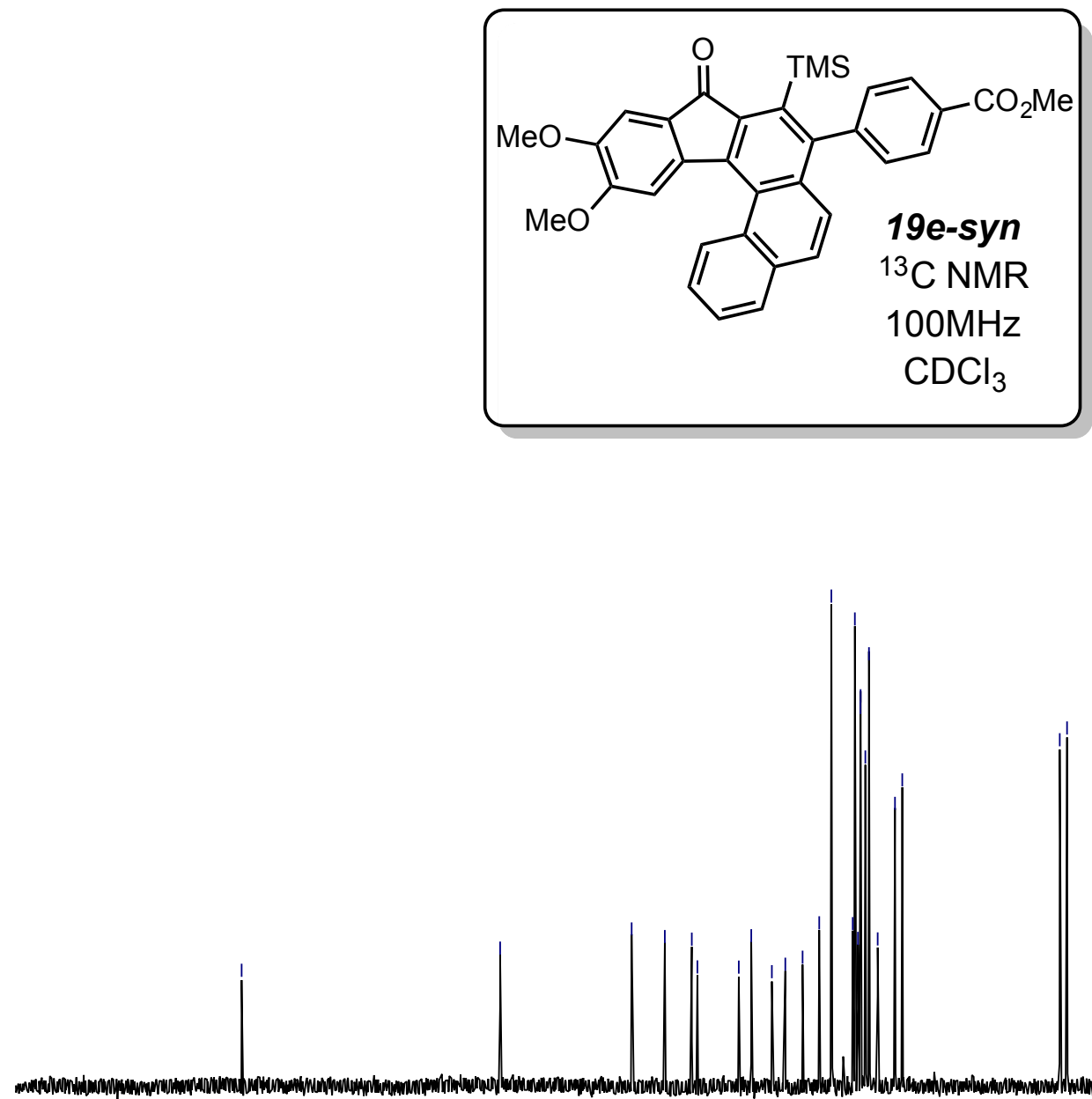

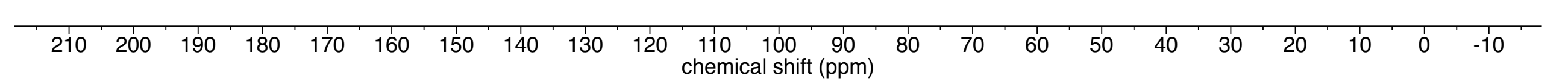


$\frac{m}{0}$

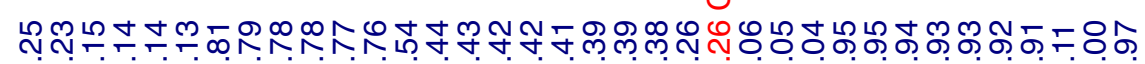

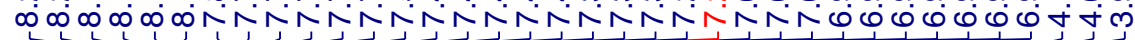
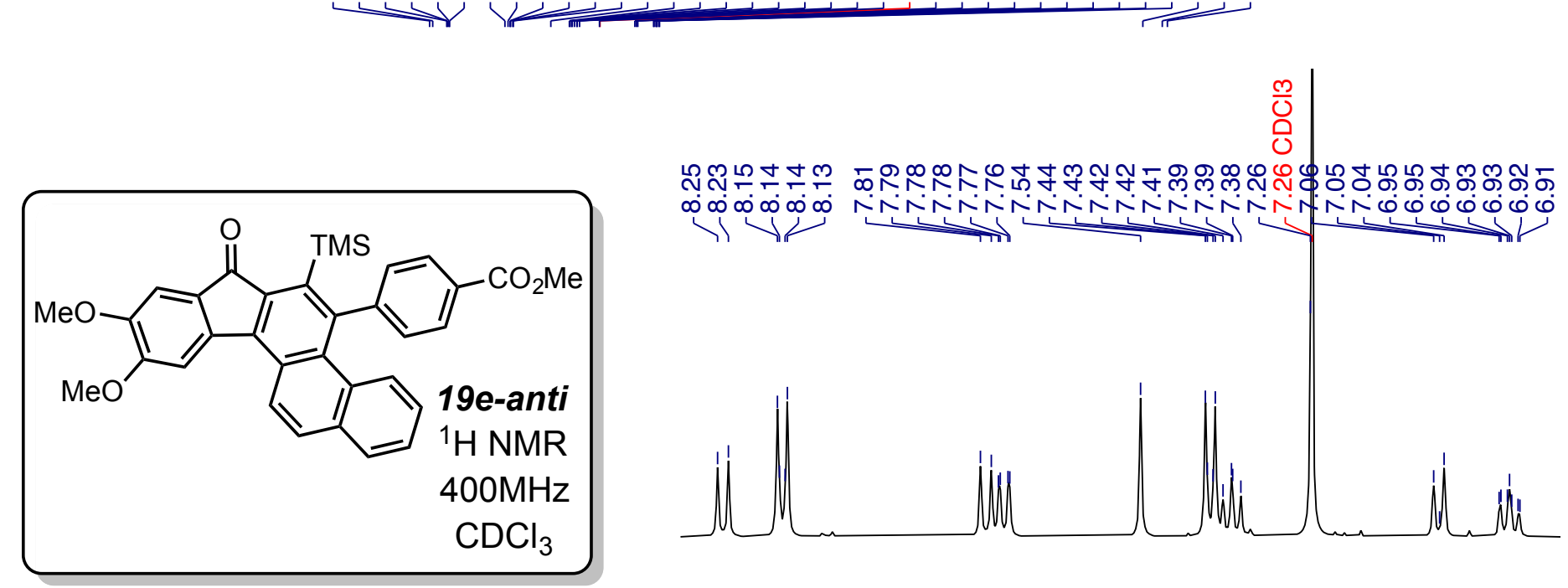

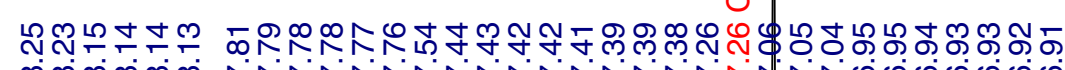
0000000 N 41 III)

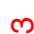

$\begin{array}{llllllllllllllllllll}8 & 8 & 8.2 & 8.1 & 8.0 & 7.9 & 7.8 & 7.7 & 7.6 & 7.5 & 7.4 & 7.3 & 7.2 & 7.1 & 7.0 & 6.9\end{array}$ chemical shift (ppm)
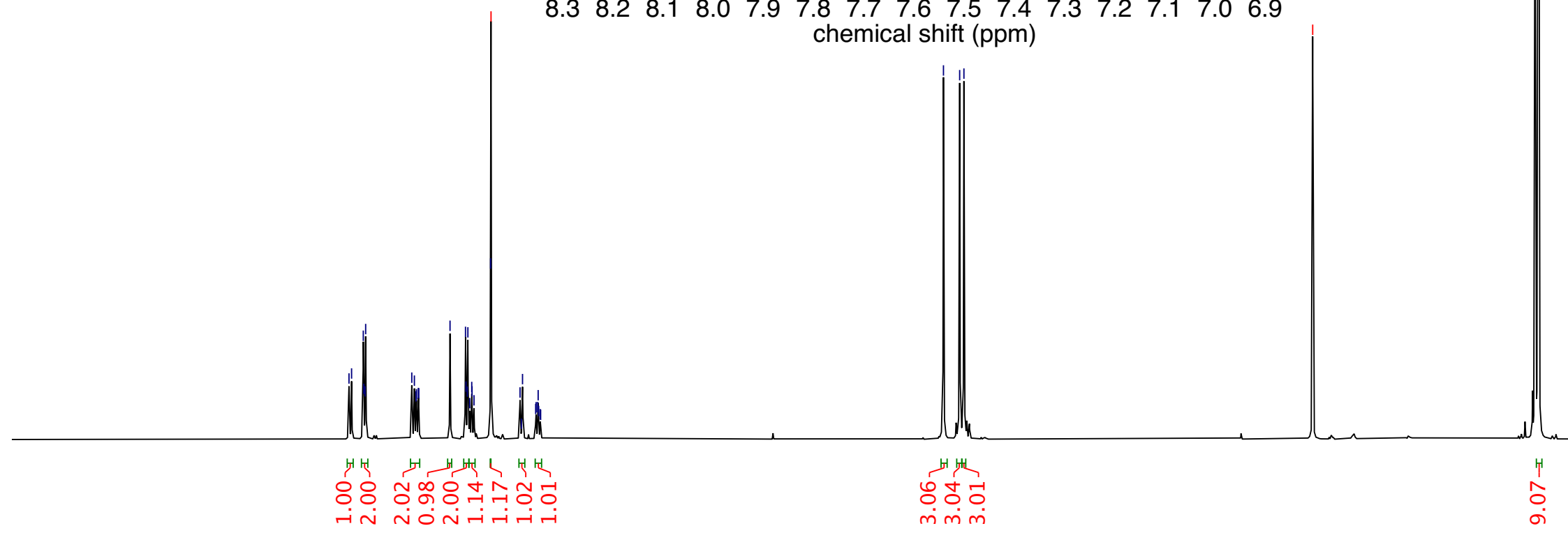


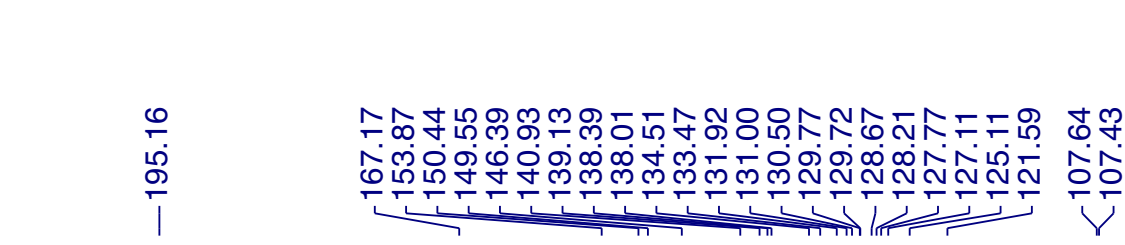
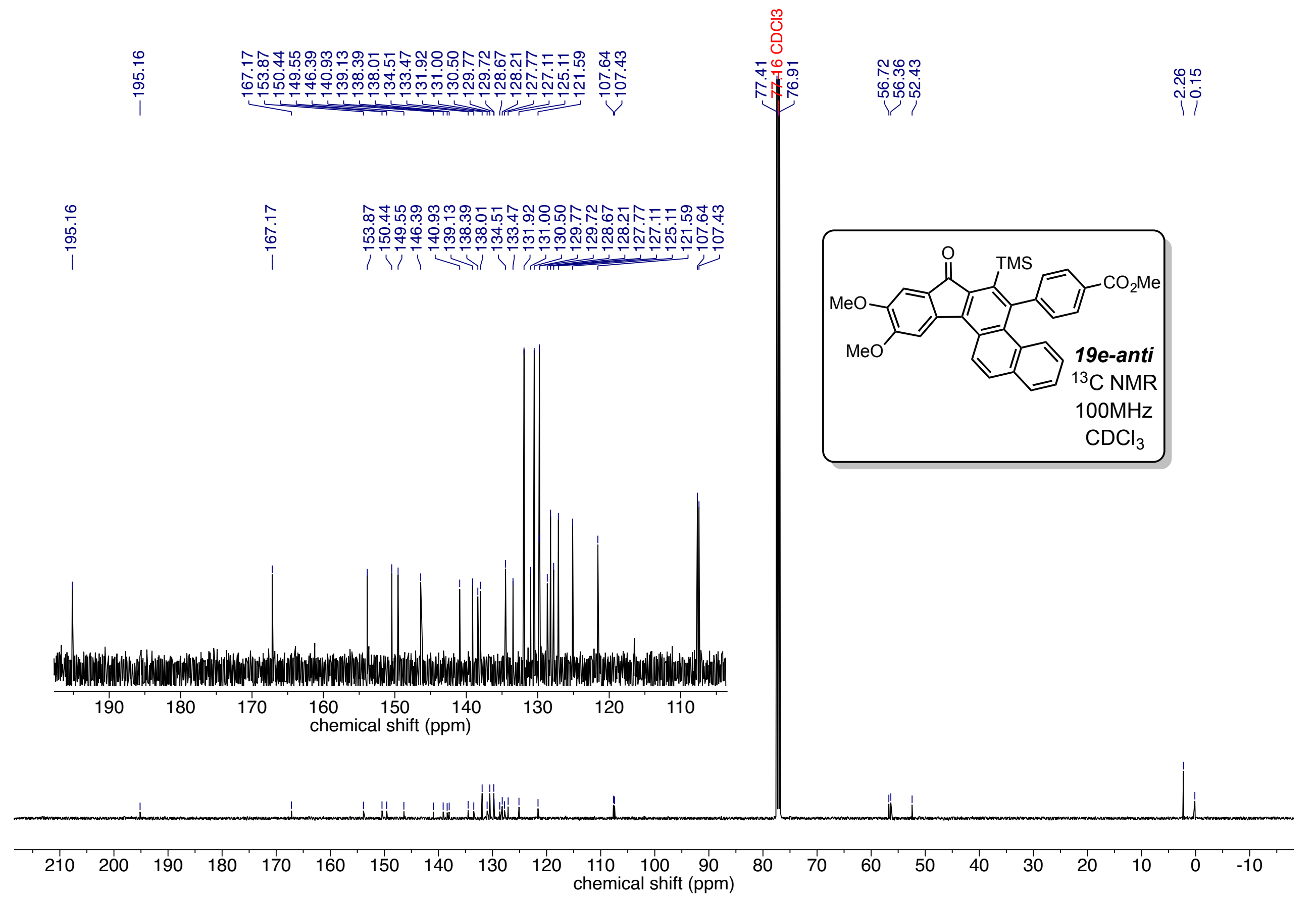


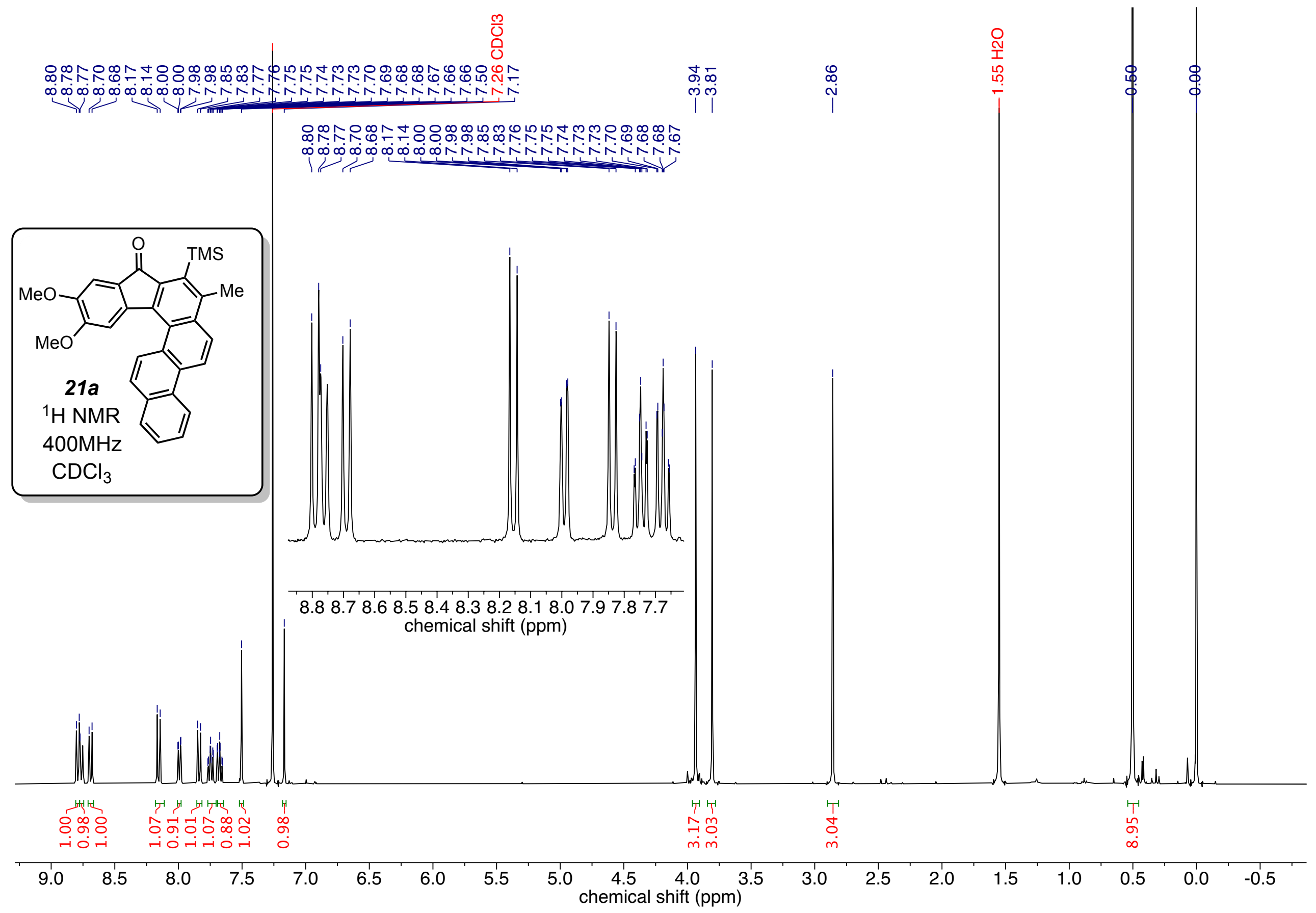



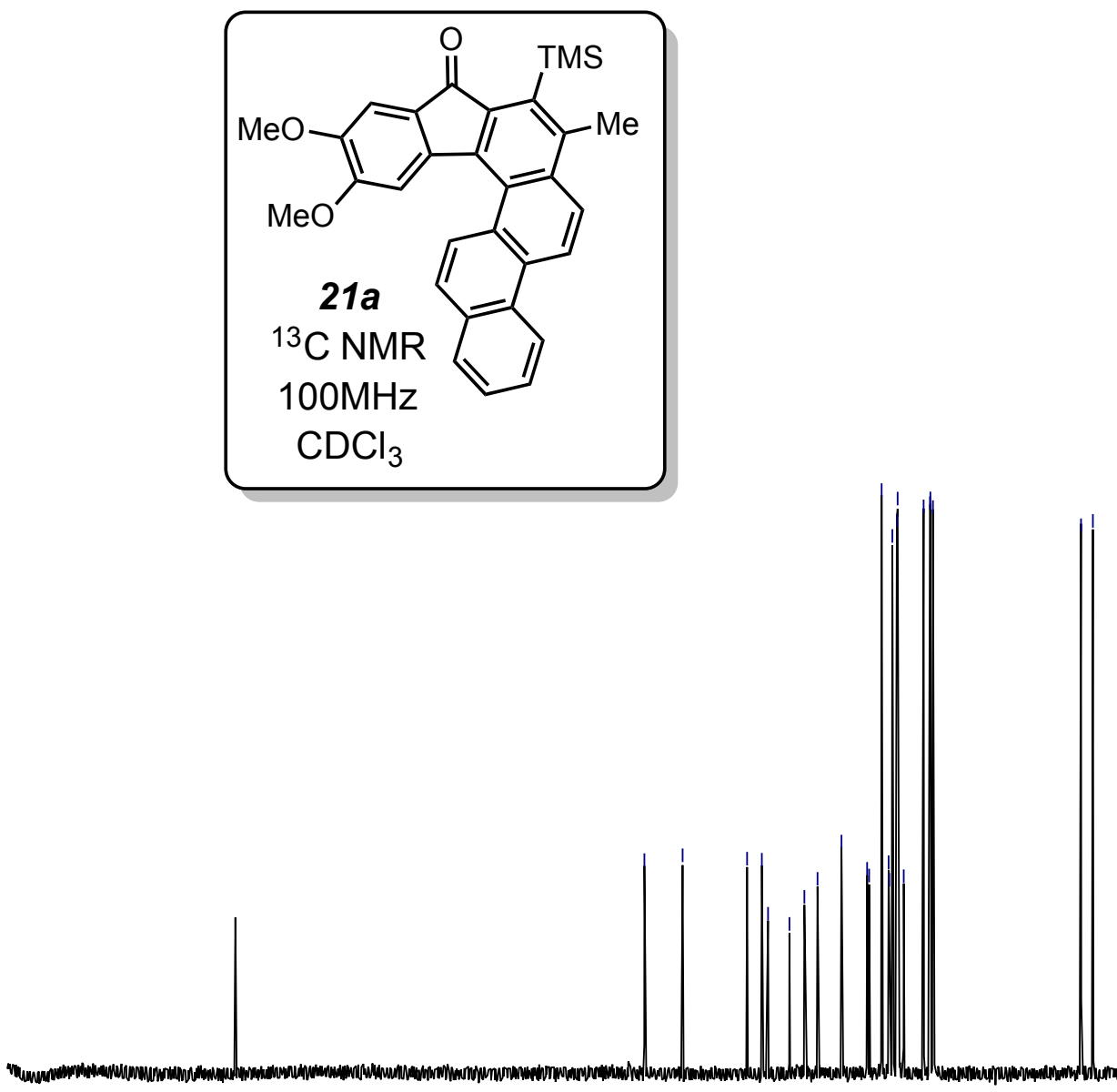


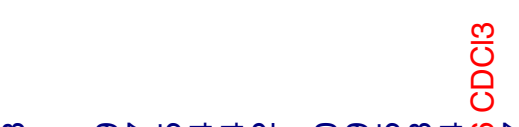

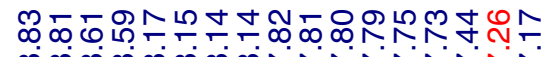
$\infty \infty \infty_{0} \infty 0_{000}$

$\checkmark 1$

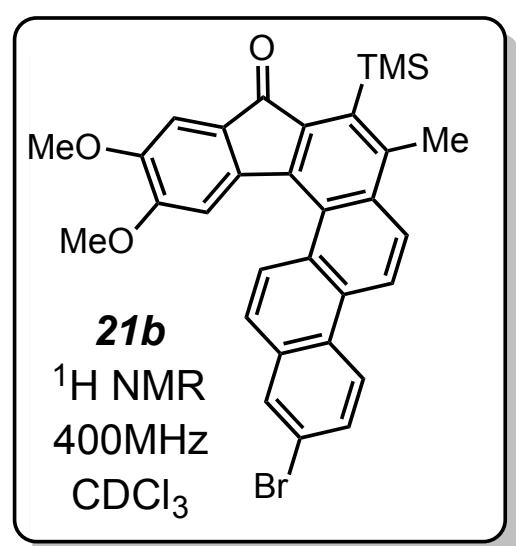

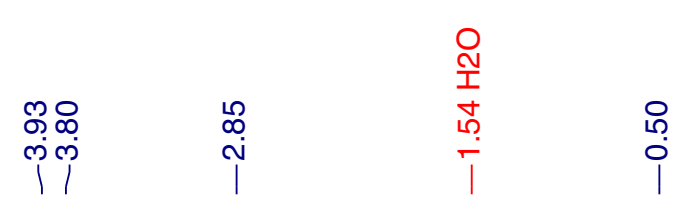

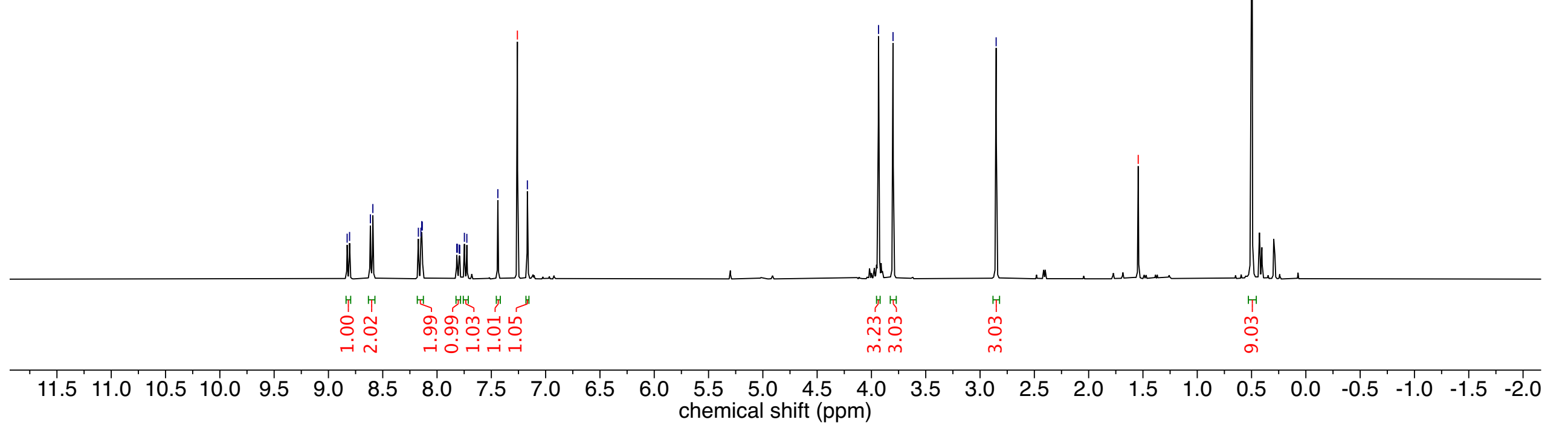




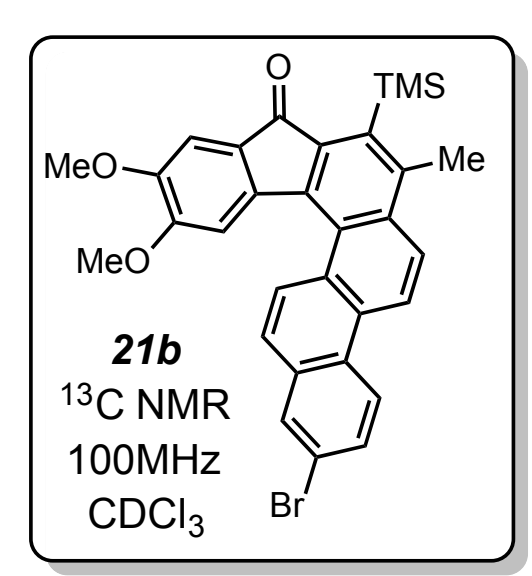

$$
-
$$

$\begin{array}{lllllllll}210 & 200 & 190 & 180 & 170 & 160 & 150 & 140 & 130\end{array}$

$80 \quad 70$




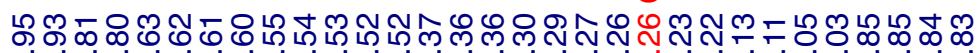

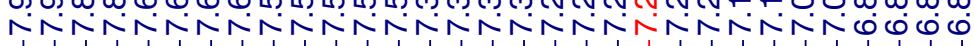

$\underbrace{2}$
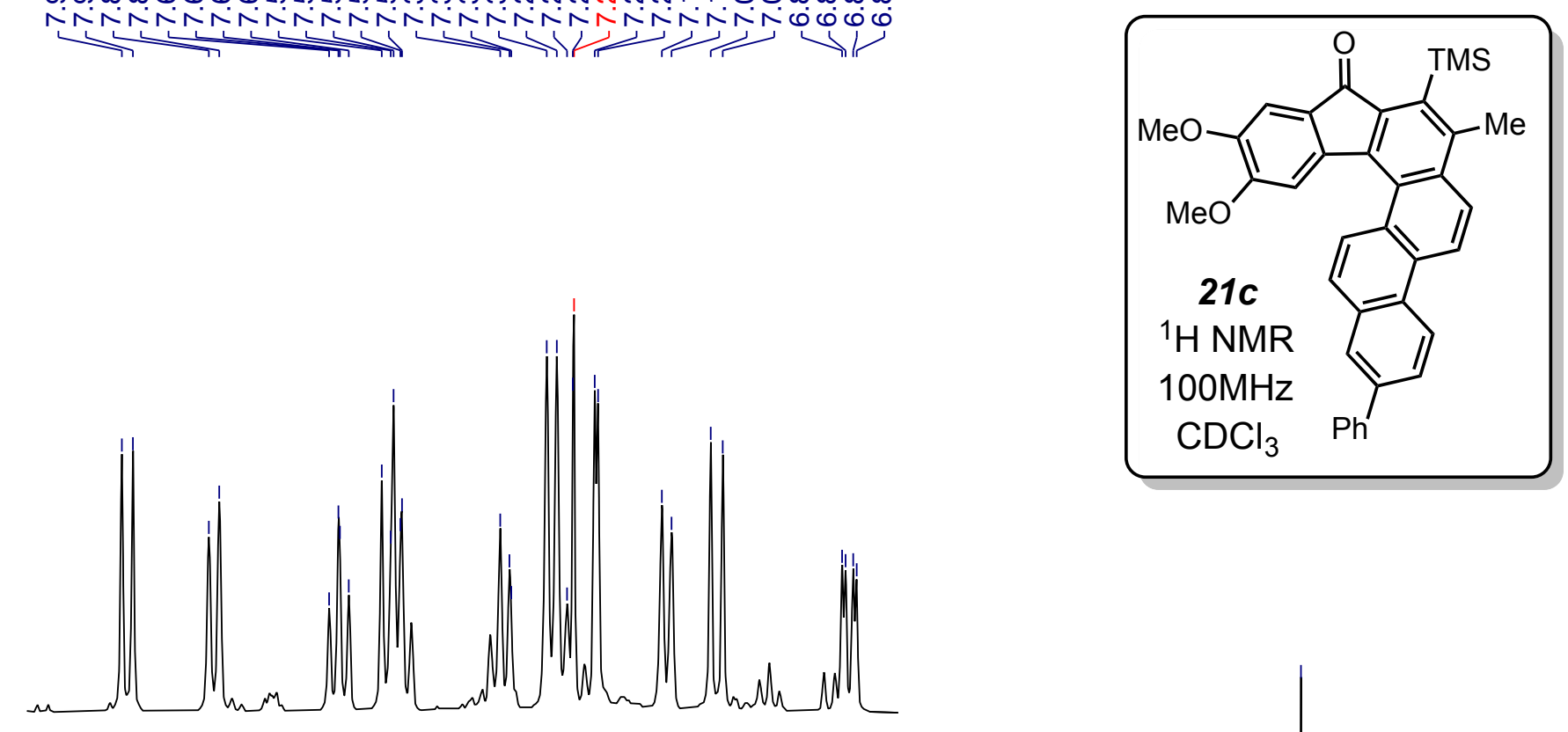

$\begin{array}{lllllllllllllllll}8.0 & 7.9 & 7.8 & 7.7 & 7.6 & 7.5 & 7.4 & 7.3 & 7.2 & 7.1 & 7.0 & 6.9 & 6.8\end{array}$ chemical shift (ppm)

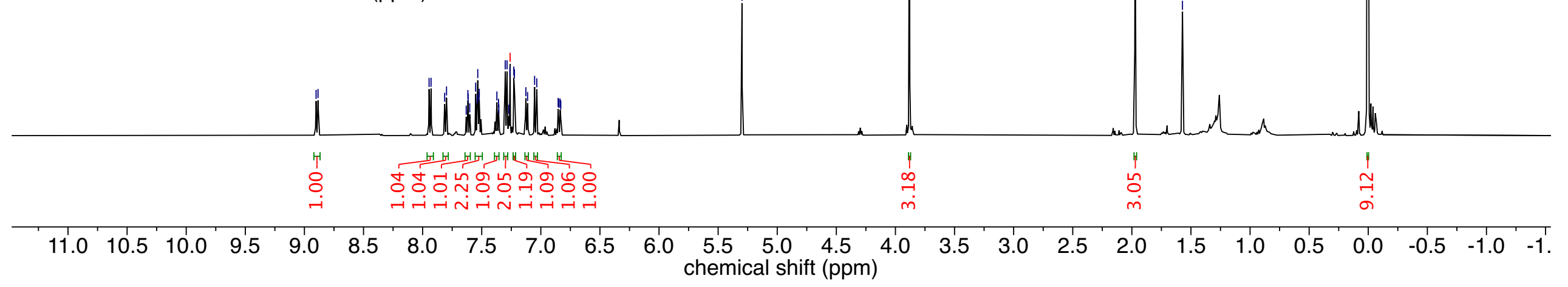




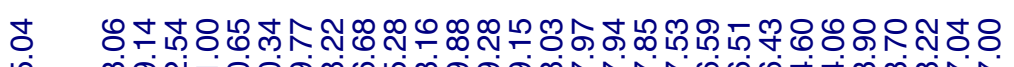

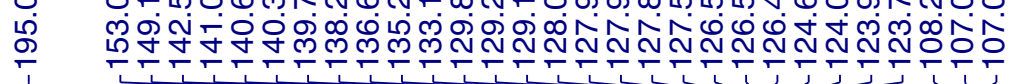

广

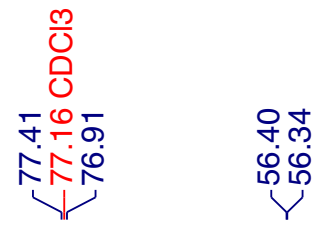

$\frac{\bar{c}}{\text { i }}$
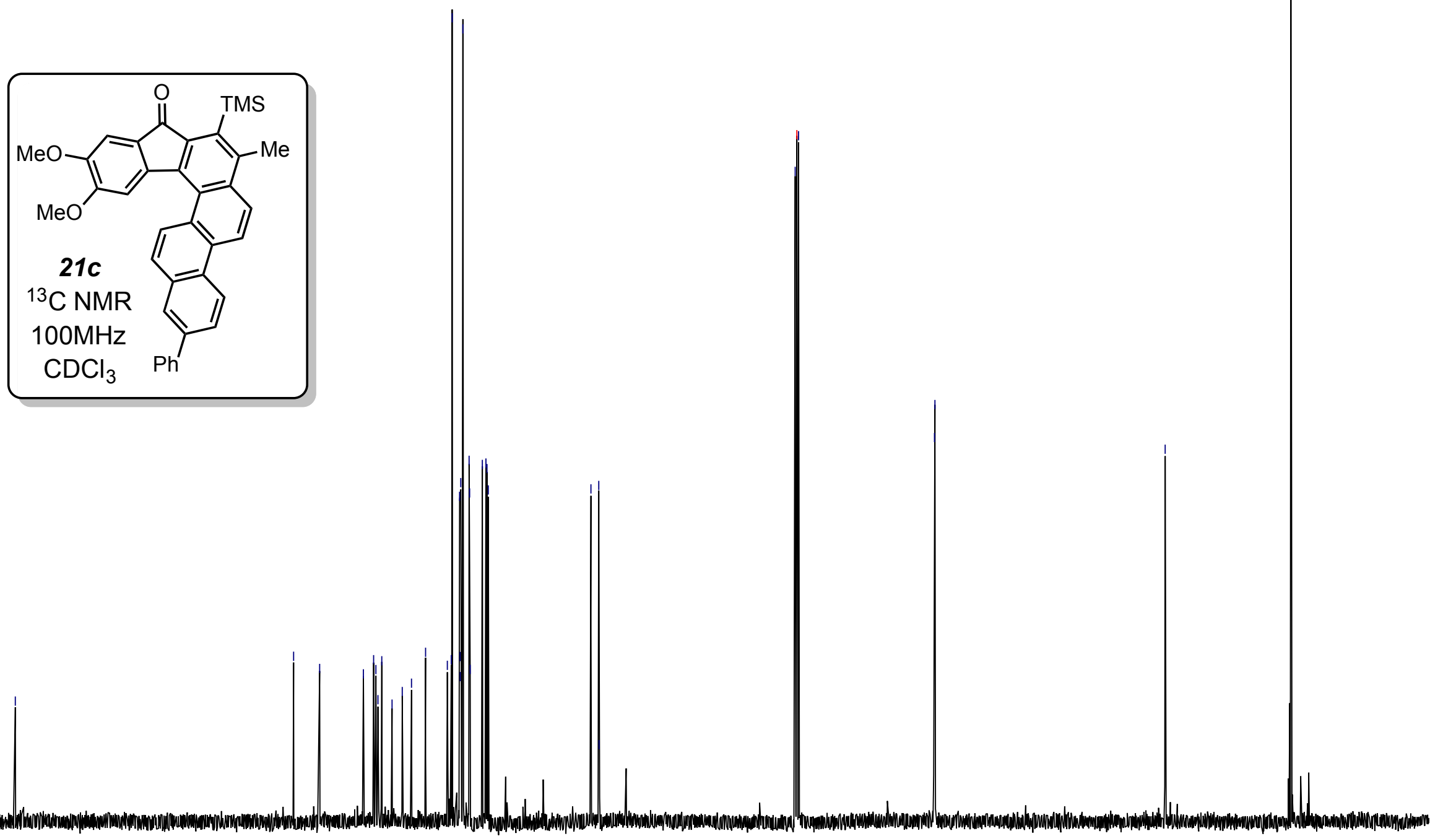

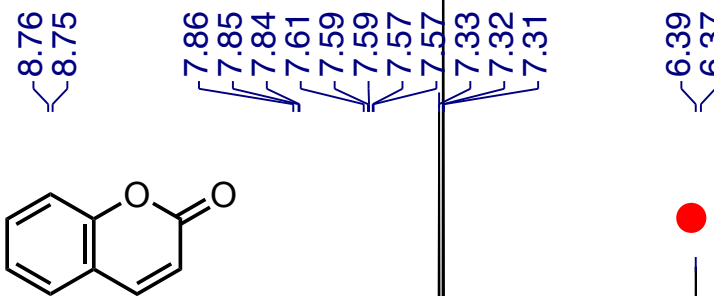

coumarin

कुल

ị

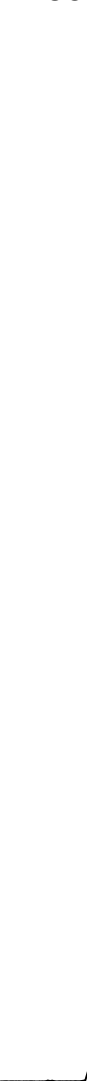

m.N

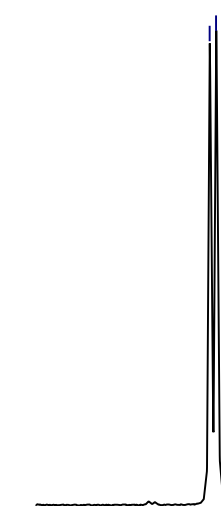

$\stackrel{000}{20}$

œ.

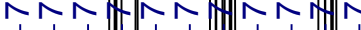

\)
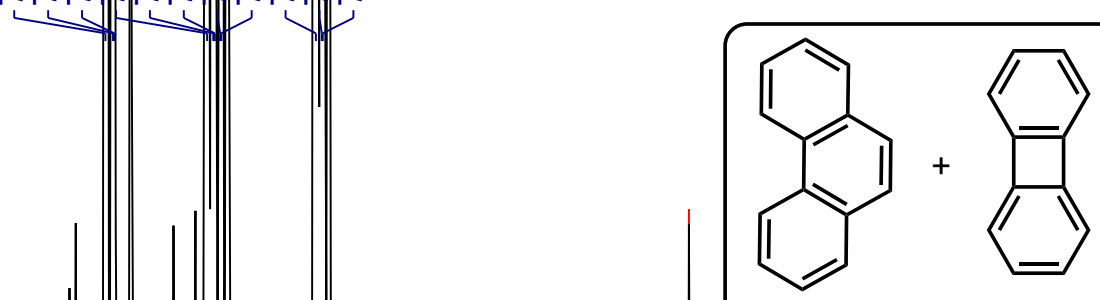

Crude 9 and 23

${ }^{1} \mathrm{H}$ NMR

$500 \mathrm{MHz}$

$\mathrm{CD}_{3} \mathrm{CN}$

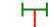

1.00

1.021 .051 .141 .11

0.410 .40

$\begin{array}{llllllllllllllll}9.2 & 9.0 & 8.8 & 8.6 & 8.4 & 8.2 & 8.0 & 7.8 & 7.6 & 7.4 & 7.2 & 7.0 & 6.8 & 6.6\end{array}$ chemical shift (ppm)

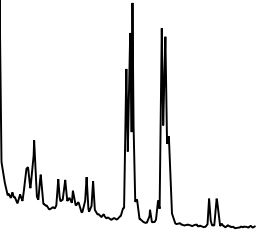

$\begin{array}{lllll}8.5 & 8.0 & 7.5 & 7.0 & 0.5\end{array}$

